\title{
Current-Induced Excitations in Ferromagnetic Single Layer And TRILAYER NANODEVICES
}

\author{
Dissertation \\ zur Erlangung des Doktorgrades \\ der Mathematisch-Naturwissenschaftlichen Fakultäten \\ der Georg-August-Universität zu Göttingen \\ vorgelegt von \\ Anne Parge \\ aus Kiel
}

Göttingen 2007 
D7

Referent: Prof. Dr. Markus Münzenberg Korreferent: PD Dr. Christian Jooß

Tag der mündlichen Prüfung: 23.03.2007 


\section{A Wish to Comply}

Did I see it go by, that Millikan mote?

Well, I said that I did.

I made a good try.

But I'm no one to quote.

If I have a defect

It's a wish to comply.

And see as I'm bid.

I rather suspect

All I saw was the lid

Going over my eye.

I honestly think

All I saw was a wink.

Robert Frost, 1949 



\section{Contents}

1 Introduction $\quad 5$

2 Background Physics $\quad 7$

2.1 Energy Bands and Magnetic Exchange Splitting . . . . . . . 7

2.2 Ferromagnetism: The Stoner Criterion . . . . . . . . . . . . 9

2.3 Spin-Dependent Conduction: The Two Current Model . . . . 10

2.4 Spin Injection into Non-Magnetic Metals: A Qualitative Approach to Spin Accumulation . . . . . . . . . . . . . 12

2.5 A Quantitative Approach to the Description of Spin Accumulation . . . . . . . . . . . . . . 13

2.5.1 The Transport Regime . . . . . . . . . . . . . . 13

2.5.2 The Valet-Fert Theory of Electrochemical Potential Splitting . . . . . . . . . . . . . . 14

2.6 Spin-Dependent Transport: The Giant Magnetoresistance Effect $($ GMR $) \ldots \ldots \ldots \ldots \ldots 16 \ldots$

2.6.1 Current in Plane and Current Perpendicular to Plane GMR ..................... 18

2.6.2 An Analogy with Polarized Light: Spin-Dependent Transmissions . . . . . . . . . . . . . 19

2.6.3 Microscopic Picture: Spin-Dependent Scattering and the Two Resistor Model of GMR . . . . . . . . . . . 19

2.6.4 The Valet-Fert Interpretation of GMR . . . . . . . . . 21

2.7 Spin Injection Into Ferromagnets: Spin Torque and Dynamics 21

2.7.1 Phenomenological Description . . . . . . . . . . 22

2.7.2 Physical Picture: Absorption of the Transverse Spin Current Component . . . . . . . . . . . . 23

2.7.3 Extending the Landau-Lifshitz-Gilbert Equation: The Effective Field and the Spin Torque Transfer Term 26

2.7.4 The Critical Current Density . . . . . . . . . . 28

2.8 Current-Induced Excitations in Ferromagnetic Single Layers . 29 
3 Experimental Equipment and Techniques 33

3.1 Sample Preparation . . . . . . . . . . . . . . . . 33

3.1.1 Nanofabrication Technique: Electron Beam Lithogra-

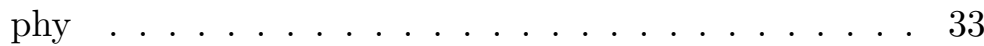

3.1.2 The Ultra High Vacuum System . . . . . . . . . . . 35

3.1.3 Sample Geometry and Preparation . . . . . . . . . . . 39

3.2 Characterization Techniques . . . . . . . . . . . . . . 41

3.2.1 Atomic Force Microscopy . . . . . . . . . . . . . . 41

3.2.2 Scanning Electron Microscopy (Including the Lithography System) . . . . . . . . . . . . . . 43

3.2.3 Transmission Electron Microscopy . . . . . . . . . . 45

3.2.4 Focused Ion Beam . . . . . . . . . . . . . . . 46

3.2.5 Experimental Setup for Transport Measurements . . . 48

3.2.6 Magnetic Photoemission Electron Microscopy . . . . . 51

4 Structural and Magnetic Characterization of the Samples 55

4.1 Characterizing the Bottom Electrodes and the Corresponding

Lithography Parameters . . . . . . . . . . . . . . 55

4.2 Cross-Sectional Views of the Nanopillars: SEM Results . . . . 60

4.3 Further Structural Analysis of the Nanopillars: TEM Results 62

4.4 The Magnetic Configuration within the Nanopillars . . . . . . 64

4.4.1 Magnetic PEEM Experiments . . . . . . . . . . . . . 64

4.4 .2 Micromagnetic Simulations . . . . . . . . . . 66

4.5 Discussion . . . . . . . . . . . . . . . . . 69

5 Current-Induced Excitations in Ferromagnetic Trilayer Samples 71

5.1 Current Sweeps at Fixed Applied Fields in the Field Perpen-

dicular Geometry . . . . . . . . . . . . . . . 71

5.1.1 Sample Resistance and Current-Dependent Background Resistance . . . . . . . . . . . . . . . 74

5.1.2 Static Excitations versus Dynamic Excitations . . . . 75

5.1 .3 Hysteretic Effects . . . . . . . . . . . . . . . 79

5.1.4 Field Dependence of the Observed Effects . . . . . . . 80

5.2 Field Sweeps at Fixed Current Bias in the Field Perpendicular Geometry . . . . . . . . . . . . . . . . . 84

5.2.1 Field-Induced Effects versus Current-Induced Effects in the Field Perpendicular Geometry . . . . . . . . . . 85

5.3 Current Sweeps at Fixed Applied Fields in the Field Parallel Geometry . . . . . . . . . . . . . . 87

5.3.1 High Resistance State at Zero Current Bias: Dipolar Coupling versus Switching to Vortex States . . . . . . 87

5.3.2 Static Excitations versus Dynamic Excitations . . . . 91

5.3.3 Excitations for Positive Current Bias . . . . . . . . . . 93

5.3.4 Field Dependence of the Observed Effects . . . . . . . 97

5.4 Field Sweeps at Fixed Current Bias in the Field Parallel Geometry . . . . . . . . . . . . . . . . . 99 
5.4.1 Field-Induced Effects versus Current-Induced Effects

in the Field Parallel Geometry . . . . . . . . . . . . 100

5.5 Discussion and Summary for the Trilayer Samples . . . . . 101

6 Current-Induced Excitations in Ferromagnetic Single Layer Samples 105

6.1 Current Sweeps at Fixed Applied Fields in the Field Perpen-

dicular Geometry . . . . . . . . . . . . . . . 106

6.1.1 Decrease of the Single Layer Resistance . . . . . . . . 106

6.1.2 Increase of the Single Layer Resistance . . . . . . . . . 110

6.1.3 Static Excitations versus Dynamic Excitations . . . . 112

6.1.4 Polarity Dependence of Single Layer Excitations . . . 113

6.1.5 Field Dependence of Single Layer Excitations . . . . . 114

6.1.6 Thickness Dependence of Single Layer Excitations . . 116

6.2 Field Sweeps at Fixed Current Bias in the Field Perpendicular Geometry . . . . . . . . . . . . . . . 117

6.2.1 The Influence of the Classical AMR Effect . . . . . . . 119

6.2.2 Current-Induced versus Field-Induced Effects in the Field Perpendicular Geometry . . . . . . . . . . . . 119

6.3 Temperature-Dependent Current Sweeps in the Field Perpendicular Geometry . . . . . . . . . . . . . . . . 121

6.3.1 The Influence of Elevated Temperatures on CurrentInduced Excitations . . . . . . . . . . . . . 122

6.4 Current Sweeps at Fixed Applied Fields in the Field Parallel

Geometry . . . . . . . . . . . . . . . 125

6.4.1 Measurements in the Field in Plane versus the Field Perpendicular to the Plane Geometry . . . . . . . . 126

6.5 Discussion and Summary for the Single Layer Samples . . . . 127

7 Concluding Remarks 129

7.1 Summary . . . . . . . . . . . . . . . . . . . . . . . . . 129

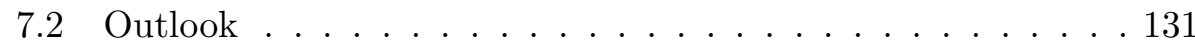

$\begin{array}{ll}\text { Bibliography } & 133\end{array}$

$\begin{array}{ll}\text { Acknowledgements } & 139\end{array}$ 
Contents 


\section{CHAPTER 1}

\section{Introduction}

The reversal of the magnetization in nanoscale magnetic structures is one of the basic operations in commercial applications of magnetism today. Examples are the manipulation of the magnetization in magnetic random-access memories (MRAMs) or in lithographically patterned ultra-high-density perpendicular recording media. In all these devices the magnetic state is switched by an external magnetic field, the so-called Oersted-Maxwell field, which is generated by an electrical current.

Due to the laws of classical electromagnetism the magnetization control by Oersted-Maxwell fields is somewhat limited by their long range and slow spatial decay. Thus new physical concepts are required in order to overcome these limits and to proceed to the nanometer length scale and the picosecond switching time scale.

A very promising concept is the application of a spin current rather than an Oersted-Maxwell field for magnetization control as proposed by Berger and Slonczewski [1, 2]. Such a spin current emerges when an electrical current is passed through a ferromagnetic layer. Then this spin-polarized current can be injected into a second ferromagnet, where the strong short-range interaction between the injected spin-polarized electrons and the localized atomic moments exerts a torque on the atomic moments and can possibly switch their direction. Since this torque has to be sufficient to perturb the magnetization from equilibrium, high current densities $\left(>10^{7} \mathrm{~A} / \mathrm{cm}^{2}\right)$, which can be obtained by a small contact size, are needed. A small contact diameter also minimizes the influence of the Oersted-Maxwell field of the electric current, which is still present but scales with $I / r$ ( $I$ : current, $r$ : radius of the magnetic structure). The exchange field induced by the spin current, on the other hand, scales with $I / r^{2}$, thus promoting the spintransfer torque effect as opposed to the effect of the Oersted-Maxwell field at nanoscale values of $r$. 
While the small structure size was realized by means of mechanical point contacts in earlier experiments [3, 4], today e-beam lithography is a standard technique to fabricate pillars with clearly defined boundaries and with diameters well below $100 \mathrm{~nm}$. In contrast to point contacts, the latter sample design guarantees more uniform current densities and reduces the problem of exchange layer coupling. Pioneering experiments on current-induced switching in ferromagnetic trilayer nanopillars were performed by $[3,4,5,6]$, addressing fundamental questions for developing a physical picture of spin torque transfer effects.

More recently, current-induced excitations have also been observed in a single ferromagnetic layer, which was sandwiched between two non-magnetic metals $[7,8,9]$. Because of the absence of a spin-polarizing layer this might appear to be a quite surprising result at first sight, but in fact spin-filtering is present even in pillar junctions with only one ferromagnetic layer. Since single layer excitations are also interesting from an application point of view, many open questions in this field are still to be investigated and answered.

Thus the main focus of this thesis was to develop a nanofabrication technique in order to produce magnetic trilayers and single layers with diameters as narrow as 100nm and below. Emphasis was also put on the installation of a sensitive transport measurement setup for the characterization of these magnetic nanopillars at low temperatures and high magnetic fields. In this way some novel results on the process of current-induced magnetization switching, which were supported by a structural analysis of the samples by means of transmission electron microscopy (TEM), could be obtained.

The thesis is organized in the following way: chapter 2 provides some physical background relevant for the understanding of the concept of current-induced switching in both magnetic single layers and trilayers. The first part of chapter 3 is devoted to the production of magnetic nanostructures including sample design, the growth techniques used, UHV equipment capabilities as well as the lithography process. Its second part summarizes the applied investigation techniques. Chapter 4 starts out with the structural analysis of the fabricated nanopillars and moves on to the results of the magnetic investigations (experiment and simulation). The results of the transport experiments performed on the ferromagnetic trilayer nanopillars are presented and discussed in chapter 5 . Thereafter first results on currentinduced excitations in ferromagnetic single layers are described in chapter 6 . These are discussed in terms of field and temperature dependence, including some phenomenological models for the observed anomalies. A summary and an outlook with suggestions for further important experiments can be found in chapter 7 . 


\section{CHAPTER 2}

\section{Background Physics}

The aim of this chapter is to introduce the physical concepts which are relevant to develop a basic understanding of current-induced excitations in a ferromagnet from an experimentalist's point of view. It starts out with the connection between the electronic states and the magnetic properties of a material, providing the basis for the explanation of the origins of ferromagnetism. Then the key concepts of spin injection and spin accumulation in non-magnetic metals are described and the most important parameters governing spindependent transport are defined. In this way the fundamentals for a short tutorial on the giant magnetoresistance effect are set. Only thereafter the more complex process of spin injection into ferromagnets and the associated spin torque effects are discussed. The chapter concludes with a brief survey on the relevant mechanisms of current-induced excitations in ferromagnetic single layers.

\subsection{Energy Bands and Magnetic Exchange Splitting}

Electrons in a solid are completely characterized by a set of quantum numbers, which comprises their energy $E$, momentum $\vec{k}$, angular symmetry and spin. This information is summarized in a plot of the band dispersions $E(\vec{k})$ with the corresponding labels for point-group symmetry and spin.

The experimentally determined bulk bands for fcc (metastable phase) and hcp Co as a representative of the ferromagnetic 3d metals are shown in figure 2.1. These diagrams were derived from a fit to photoemission and inverse photoemission data $[10,11]$.

As a result of ferromagnetic exchange splitting, the bands are divided into two subsets: one with majority spin $(\uparrow)$, the other one with minority $\operatorname{spin}(\downarrow)$. It is striking that the Co majority $\mathrm{d}$ band is totally filled with electrons, while the d minority band is essentially above the Fermi Energy $E_{F}$ and 

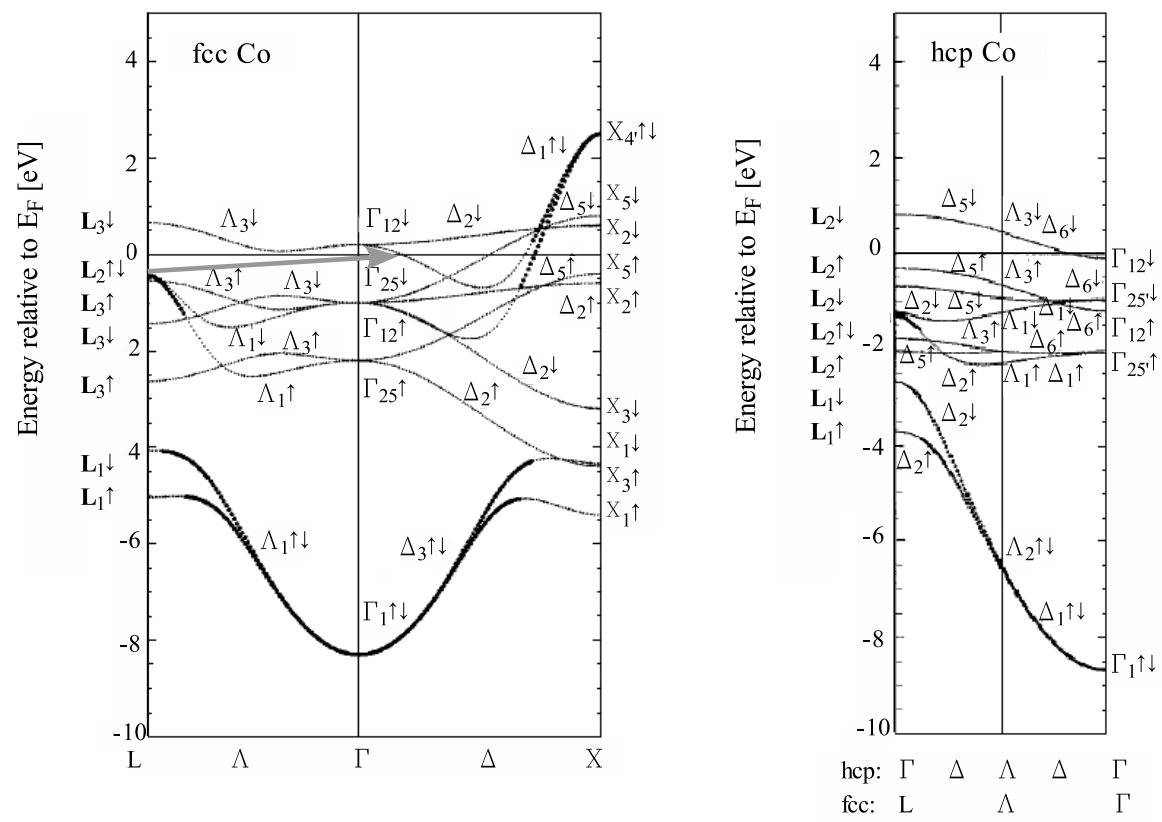

Figure 2.1: Empirical fcc and hcp Co bulk band structures obtained from a fit to photoemission data. Bands with s,p character are highlighted by bold symbols. The gray arrow in the left panel indicates a spin-flip process, which requires the energy of the Stoner gap. Adapted from [10, 11].

thus only partly occupied. This spin imbalance is the key to magnetism and gives rise to a magnetic moment. At this point it is important to note that most of the moment is carried by the $3 \mathrm{~d}$ electrons, while the $\mathrm{s}$ and $\mathrm{p}$ electrons are weakly polarized in the opposite direction. The physical origin of the exchange splitting and its consequences will be further illuminated in section 2.2 .

Figure 2.2 demonstrates that $E(\vec{k})$ plots can also exemplify two other important aspects of exchange splitting: single and collective electron excitations. The fundamental single electron excitation is a spin flip process, in which an electron is lifted from the fully occupied majority d band into the unoccupied part of the minority $d$ band. The minimum energy required for this process is the energy of the Stoner gap $\Delta$, which can be extracted from figure 2.1: $\Delta=0.35 \mathrm{eV}$ for fcc Co (from $E_{F}$ to L3), which involves momentum transfer. If no momentum is transferred, the energy of the spin flip excitations equals the energy of the exchange splitting.

The fundamental collective electron excitation is the so-called spin wave, which is also referred to as a magnon. Spin waves are low energy excitations starting out with a parabolic energy dispersion at $\vec{k}=\overrightarrow{0}$ and broaden upon overlapping with the single electron excitations above the Stoner gap. Due to the altered boundary conditions special spin wave modes can emerge at surfaces or in multilayer systems [13]. 

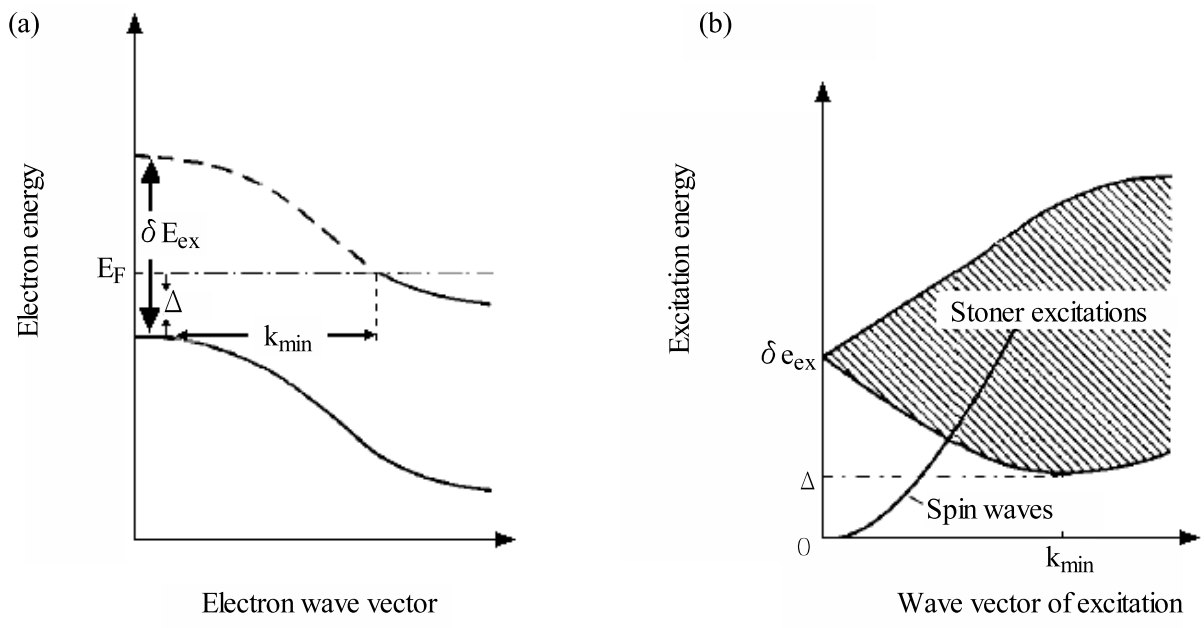

Figure 2.2: (a) $E(\vec{k})$ diagram for single electron excitations with energies above the Stoner gap: spin flip process. (b) $E(\vec{k})$ diagram for distinguishing between lower energy collective excitations (spin waves) and Stoner excitations [12].

\subsection{Ferromagnetism: The Stoner Criterion}

As it has been outlined in the previous section, the magnetic properties of a material reflect its underlying bandstructure, which is essentially determined by the exchange-split $d$ band in ferromagnetic 3d metals. Since the $d$ electrons are highly localized, they experience a strong Coulomb repulsion force provided that they occupy the same orbital with antiparallel spins. In conjunction with the Pauli exclusion principle, which does not allow two electrons with the same spin on the same orbital, a parallel orientation of the spins leads to a reduction of the Coulomb interaction, because then different orbitals have to be occupied. In this situation the ferromagnetic exchange interaction lowers the energy of the system and thus favors the formation of a spontaneous magnetic moment. On the other hand arranging all electrons with parallel spin also raises the kinetic energy; the increase in kinetic energy being the bigger the wider the $d$ band. Hence, there are two competing tendencies which have to be balanced in order to find out if ferromagnetic exchange splitting and thus ordering is beneficial. The following condition, the so-called Stoner criterion [15], is used as an indicator for the appearance of ferromagnetism:

$$
D\left(E_{F}\right) \cdot I>1 .
$$

Here $D\left(E_{F}\right)$ represents the density of states per spin and atom at the Fermi energy and $I$ is the exchange integral. As figure 2.3 shows, the condition is fulfilled for Fe, Co and Ni.

For these transition metals the total energy can be reduced by bringing enough electrons down in energy by opening up the ferromagnetic exchange splitting. The resulting magnetic moment is $1.72 \mu \mathrm{B}$ per atom for Co. 


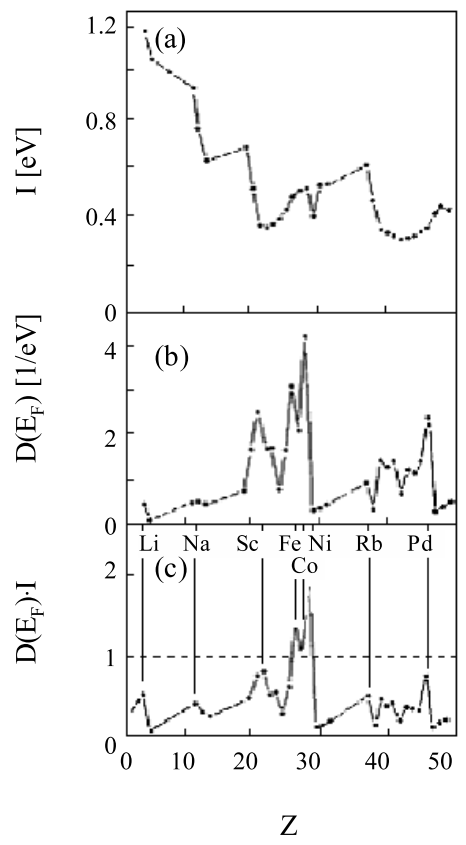

Figure 2.3: The Stoner criterion: exchange integral (a), density of states (b) and their product (c) as a function of the atomic number $Z$ (adapted from [12]).

\subsection{Spin-Dependent Conduction: The Two Current Model}

The two current model developed by Mott in the 1930s [14] assumes that the conductivity of a metal $\sigma$ is the sum of the basically independent conductivities for spin-up and spin-down electrons:

$$
\sigma=\sigma_{\uparrow}+\sigma_{\downarrow}
$$

This concept implies that there is hardly any exchange between the two spin channels, at least on the short time scales defined by all other processes in the system. In fact, spin flip incidents do occur in every real material, but they are comparatively rare, so that in most cases they can be neglected when considering the dissipative processes which give rise to electrical resistivity.

The independence of the two families of charge carriers is one of the two essential ingredients for the discussion of spin-dependent transport properties. The other one is the ferromagnetic exchange interaction, which has already been described above (see section 2.2).

This situation becomes clearer upon examining figure 2.4, where the density of states for non-magnetic $\mathrm{Cu}$ can be compared to the one of ferromagnetic Co. The conductivity is determined by the position of the Fermi energy with respect to the $\mathrm{d}$ bands. For $\mathrm{Cu}$, the $\mathrm{d}$ bands are totally filled 
with electrons and the Fermi level lies well within the sp band for both spin-up and spin-down electrons (bottom of figure 2.4). Since the electron velocity in the sp band is comparatively high and the low density of states guarantees a low probability of scattering, the mean free path of electrons is long. Thus $\mathrm{Cu}$ is a very good conductor. Due to the exchange splitting, there is a different situation for ferromagnetic Co: the majority d band is fully occupied, while the minority d band is only partly filled with electrons. For this reason the Fermi level lies within the sp band for majority spins, but only within the $\mathrm{d}$ band for minority spins. Hence, the conductivity is high for majority electrons (due to the same reasons as the ones given for the high conductivity of $\mathrm{Cu}$ ). The contrary holds true for minority-spin electrons: here the bands are strongly spd hybridized and show a high density of states but a low electron velocity due to the strong localization of the d states. Hence, the mean free path for minority electrons is rather short, leading to a low minority spin conductivity.

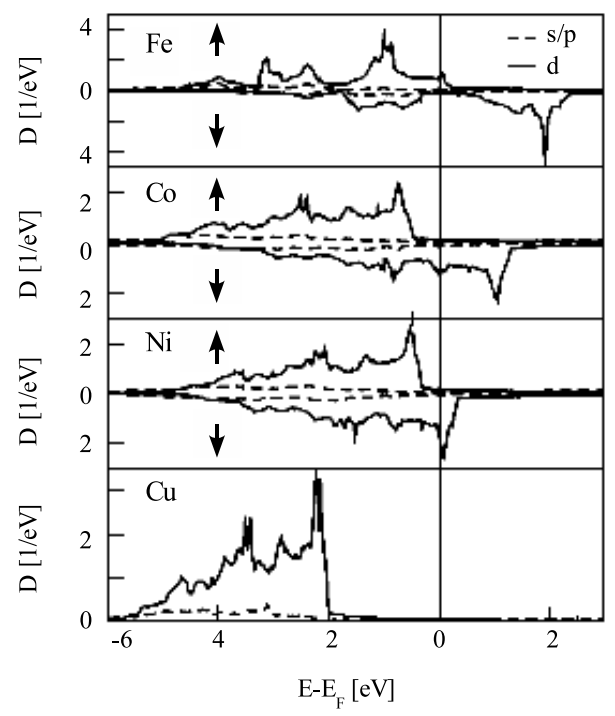

Figure 2.4: Density of states for the ferromagnetic metals $\mathrm{Fe}, \mathrm{Co}, \mathrm{Ni}$ compared to the one of non-magnetic Cu. The Fermi energy corresponds to zero energy [16].

This strong spin asymmetry of the conductivity in bulk ferromagnets results in a spin-dependent transmission of electrons in the presence of an interface between a ferromagnetic and a non-magnetic metal: if an electrical current flows through a ferromagnet, this current is primarily mediated by high mobility charge carriers, which are the majority electrons in the case of Co. As a result the current transmitted to the non-magnet yields more majority than minority electrons, i.e. the current is spin polarized. Ferromagnetic elements may thus be used as spin-polarized current sources in electric circuits. This phenomenon will be further elucidated in the following section. 


\subsection{Spin Injection into Non-Magnetic Metals: A Qualitative Approach to Spin Accumulation}

When a current is passed from a ferromagnet into a non-magnetic material, it brings along an injection of net spin angular momentum (see figure 2.5 (a)) due to the spin-dependent conductivity as it has already been discussed in chapter 2.3. However, in the normal metal the conductivities for both spin orientations are equal, so that a net magnetization aligned parallel to the magnetization in the ferromagnet is induced in the non-magnet. The buildup of this magnetization close to the interface is also referred to as spin accumulation. This process is primarily determined by the dynamic equilibrium between the net spin injection rate and the spin-flip rate in the non-magnet. As a consequence the spin accumulation decays exponentially away from the interface on a distance called the spin diffusion length $\lambda_{s d}$.
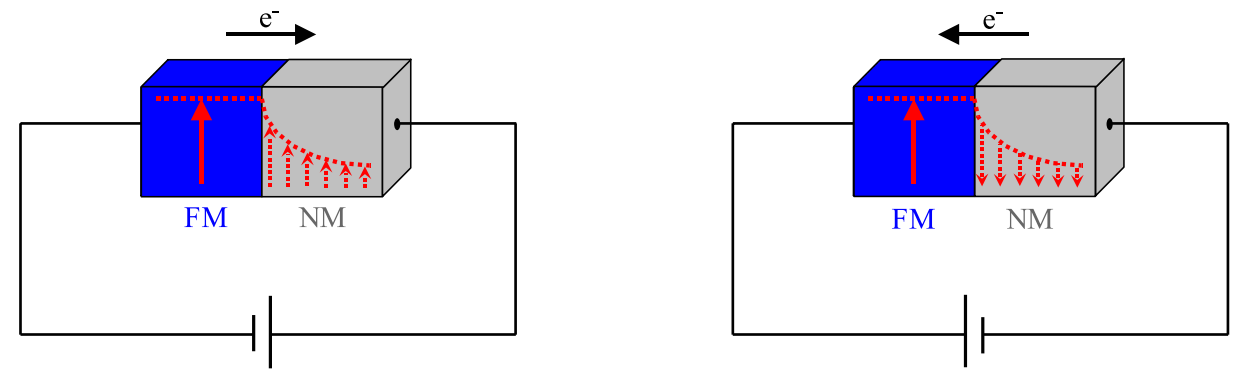

Figure 2.5: Illustration of the spin accumulation at the ferromagnetic/non-magnetic interface. Note the opposite sign of spin accumulation for reversed current flow.

So far only the situation illustrated in figure 2.5 (a) has been taken into account, which refers to the electrons travelling from the ferromagnet into the non-magnet. If the direction of current is reversed, i.e electrons pass through the normal metal before entering the ferromagnet (figure 2.5 (b)), a similar process can be observed: due to the different reflection and transmission coefficients for majority and minority electrons at the interface one spin species is predominantly transmitted, while the other one is predominantly reflected. The reflected spin species again causes an injection of net angular momentum into the normal metal, but this time the spin accumulation has a magnetization direction opposite to the one of the ferromagnetic layer.

Since the spin diffusion length plays a crucial role in the experiments performed in this thesis, it is beneficial to calculate a rough estimate of its magnitude. A newly injected spin undergoes a number of $N$ momentum changing collisions in the non-magnet before being flipped to the opposite spin direction after an average time of $\tau_{s f}$. The average distance between two collisions equals the mean free path $\lambda_{m f p}$. By analogy with a random walk in three dimensions the average distance the spin penetrates into the 
non-magnet can be estimated to be

$$
\lambda_{s d}=\lambda_{m f p} \cdot \sqrt{\frac{N}{3}}
$$

Furthermore, the total distance covered by the spin $N \cdot \lambda_{m f p}$ is equal to its Fermi velocity $v_{F}$ times the spin-flip time:

$$
N \cdot \lambda_{m f p}=v_{F} \cdot \tau_{s f} .
$$

Combining equations 2.3 and 2.4 yields the spin diffusion length

$$
\lambda_{s d}=\sqrt{\frac{v_{F} \cdot \tau_{s f} \cdot \lambda_{m f p}}{3}} .
$$

For a spin flip time of $11 p s$ [17], a mean free path of $192 \AA$ [18] and a Fermi velocity of $1.57 \cdot 10^{6} \mathrm{~m} / \mathrm{s}$ [19] substitution leads to a $\mathrm{Cu}$ spin diffusion length of $332 \mathrm{~nm}$ at room temperature. This estimated value is in excellent agreement with the experimental result of $350 \mathrm{~nm} \pm 50 \mathrm{~nm}$ by Jedema et al. [17].

\subsection{A Quantitative Approach to the Description of Spin Accumulation}

The previous section describes how a spin current arises in a rather phenomenological approach. Here the same issue is addressed from a theoretical point of view, which means following the charge and spin transport throughout the whole nanodevice.

\subsubsection{The Transport Regime}

Charge and spin transport can occur in three different regimes, which in return determine the appropriate formalism to describe the situation: quantum mechanically coherent, ballistic or diffusive transport.

Quantum size effects have to be taken into account when dealing with system dimensions in the range of the Fermi wave length $\lambda_{F}$. Since $\lambda_{F}$ only amounts to $1-2 \AA$, this does not really apply for the samples studied within this thesis, which have a minimum layer thickness $t$ of $3 \mathrm{~nm}$ and much larger lateral dimensions of approximately $80 \mathrm{~nm}$. Besides, interface roughness, thickness variations from layer to layer, intermixing and impurities at interfaces make quantum mechanically coherent scattering from the interfaces of a layer very unlikely [20].

Still, the transport could be ballistic. Then the electrons do not scatter when they propagate from one interface to the other. At first view this might be true, because their mean free path $\lambda_{m f p}$ is much larger than the thickness of the individual layers $t$. However, the transport properties are strongly determined by the density of scattering centers in the entire plane of each layer. As indicated above, the interfaces in real samples are in 
general far from perfect, so that the transport is likely to be governed by disordered interface areas, where $\lambda_{m f p}$ is much shorter than in the bulk material. Thus it is justifiable to assume that at least one dimension $w$ of the analyzed system is much larger than the mean free path: $w \gg \lambda_{m f p}$. Hence, transport does not predominantly take place in the ballistic, but in the diffusive regime. In this regime the high concentration of scattering centers requires the averaging of the scattering potential. The averaging leads to the loss of the memory of the electron momentum direction, which results in an additional resistance.

\subsubsection{The Valet-Fert Theory of Electrochemical Potential Splitting}

Here a brief review of the basic equations for spin transport across a ferromagnetic/normal metal interface in the diffusive regime will be given. The description relies on a separate definition of the electrochemical potentials for both spin directions as it was first introduced by Son et al. [21]. Based on the Boltzmann transport equation, this approach has been developed further by Valet and Fert for all metal multilayers in the context of the giant magnetoresistance [22]. For a far more detailed review on the semiclassical Boltzmann approach and its applications to transport problems in solids the reader is referred to [23, 24].

Upon excluding diffusion processes parallel to the interfaces, the electric transport can be simplified to a one dimensional problem with the electrical current $J$ flowing in the $x$-direction perpendicular to the thin film planes. Then the overall particle current $J_{n}$ can be expressed as the sum of the electric field driven current $J_{E}$ and the diffusion current $J_{D}$. This leads to a well-known relation between the electric field $E$ and the number of charge carriers $n$ :

$$
J_{n}=J_{E}+J_{D}=\sigma \frac{E}{e}-D \frac{\partial n(x)}{\partial x} .
$$

Here $\sigma$ denotes the conductivity, which is linked to the diffusion constant $D$ via the Einstein relation

$$
\sigma=e^{2} \rho\left(E_{F}\right) D
$$

where $\rho\left(E_{F}\right)$ represents the density of states at the Fermi energy $E_{F}$.

It is convenient to rewrite equation 2.6 in terms of the electrochemical potential $\mu$, which is obtained by adding the chemical energy $\mu_{c h}$ and the potential energy $\mu_{p o t}=e \cdot V$. With this it follows from equation 2.6 that

$$
J_{n}=-D \rho\left(E_{F}\right) \frac{\partial\left(\mu_{p o t}\right)}{\partial x}-D \rho\left(E_{F}\right) \frac{\partial\left(\mu_{c h}\right)}{\partial x}=-D \rho\left(E_{F}\right) \frac{\partial \mu}{\partial x} .
$$

Now the continuity equation for the induced magnetization $m=\mu_{B}\left(n_{\uparrow}-\right.$ $\left.n_{\downarrow}\right)$ is introduced:

$$
\frac{\partial m}{\partial t}+\frac{\partial J_{m}}{\partial x}=-\frac{2 m}{\tau_{s f}}
$$


where $J_{m}$ represents the magnetization current given by $J_{m}=\mu_{B}\left(J_{n \uparrow}-J_{n \downarrow}\right)$. In dynamic equilibrium the induced magnetization $m$ is constant, so that in this situation the equation above transforms to

$$
-\mu_{B} \frac{\partial}{\partial x}\left(J_{n \uparrow}-J_{n \downarrow}\right)=\frac{2 \mu_{B}\left(n_{\uparrow}-n_{\downarrow}\right)}{\tau_{s f}} .
$$

Substituting equation 2.8 in this relation and including the identity

$$
n_{\uparrow}-n_{\downarrow}=\rho\left(E_{F}\right)\left(\mu_{\uparrow}-\mu_{\downarrow}\right)=\rho\left(E_{F}\right) \Delta \mu
$$

yields the following diffusion equation, which is the fundamental equation to describe spin-dependent transport:

$$
D \frac{\partial^{2}(\Delta \mu)}{\partial x^{2}}=\frac{2(\Delta \mu)}{\tau_{s f}} .
$$

This differential equation has exponential solutions of the form

$$
\Delta \mu=B e^{x / \lambda_{s d}}+C e^{-x / \lambda_{s d}} .
$$

The constants $B$ and $C$ are defined by the boundary conditions at the interface between the ferromagnet and the non-magnetic metal. In the absence of an interface resistance and spin flip scattering at the interface, the boundary conditions are given by the continuity of the electrochemical potentials $\mu_{\uparrow}$ and $\mu_{\downarrow}$ at the interface as well as the conservation of currents $J_{n \uparrow}$ and $J_{n \downarrow}$ across the interface. The spin diffusion length can be calculated by the random walk treatment yielding $\lambda_{s d}^{N M}=\left(D \tau_{s f} / 2\right)^{1 / 2}$ for the nonmagnetic metal, where the mobility of the charge carriers is the same for spin up and spin down. However, in the spin asymmetric ferromagnet $D_{\uparrow}$ and $D_{\downarrow}$ are different and thus also the term for $\lambda_{s d}$ has to be modified:

$$
\lambda_{s d}^{F M}=\sqrt{\frac{\tau_{s f}}{1 / D_{\uparrow}+1 / D_{\downarrow}}} .
$$

Figure 2.6 visualizes the solutions of equation 2.12 for a single interface between $\mathrm{Co}$ and $\mathrm{Cu}$. In contrast to what has been stated in the previous paragraph, here the conservation of currents in each spin channel has been replaced by the following boundary conditions, which take spin-dependent scattering events directly at the interface into account:

$$
\begin{aligned}
& \left.\mu_{\uparrow}\right|_{-0}-\left.\mu_{\uparrow}\right|_{+0}=e R_{B}^{*}(1-\gamma) J_{n \uparrow}, \\
& \left.\mu_{\downarrow}\right|_{-0}-\left.\mu_{\downarrow}\right|_{+0}=e R_{B}^{*}(1+\gamma) J_{n \downarrow},
\end{aligned}
$$

where $R_{B}^{*}$ is the total interface resistance and $\gamma$ the spin asymmetry coefficient. The graph reveals, that spin flip processes at the interface give rise to a discontinuity of the electrochemical potentials of the two spin species. Furthermore, the exponential decay of the spin accumulation $\Delta \mu$ away from the interface can be clearly observed. Due to the very different spin diffusion lengths of $\mathrm{Cu}$ and $\mathrm{Co}$, the distance of this decay varies. 


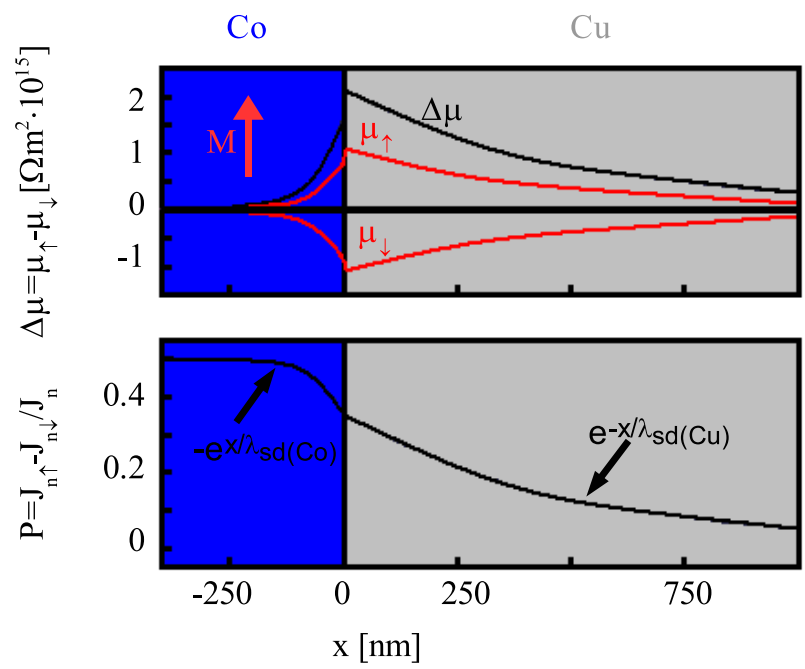

Figure 2.6: Top: spin accumulation $\Delta \mu=\mu_{\uparrow}-\mu_{\downarrow}$ at the interface of $\mathrm{Co} / \mathrm{Cu}$. Bottom: variation of the current spin polarization. The curves have been calculated with parameters derived from [25, 26, 27] and the spindependent resistances for $\mathrm{Co}$ and $\mathrm{Cu}$ (adapted from [28]).

\subsection{Spin-Dependent Transport: The Giant Magnetoresistance Effect (GMR)}

Having outlined the basic mechanisms of spin accumulation at the interface of a ferromagnetic/non-magnetic double layer, a simple metallic trilayer device as the one depicted in figure 2.7 can now be discussed. In 1988 Baibich et al. observed that the application of an external magnetic field to an $\mathrm{Fe} / \mathrm{Cr} / \mathrm{Fe}$ trilayer results in a significant reduction of the electrical resistance of the sample [29]. An even bigger effect was simultaneously discovered for $\mathrm{Fe} / \mathrm{Cr}$ multilayers [30]: at $4.2 \mathrm{~K}$ the resistivity of the multilayer in an external magnetic field dropped by almost $50 \%$. Since this effect was much larger than any other magnetoresistive effect that had been observed at that time, it was called giant magnetoresistance (GMR).

The mechanism of GMR is attributed to the change of the relative magnetization directions of the two (or more) ferromagnetic layers, which is induced by the external magnetic field. In order to allow for this reorientation, the layers have to be separated from one another by a sufficiently thick non-magnetic spacer layer, so that their magnetizations are not strongly coupled. An antiparallel orientation of the magnetization (see upper inset of figure 2.8) then results in a high resistance, while a parallel configuration (see lower insets of figure 2.8) shows a relatively low resistance. The GMR ratio is then defined as

$$
G M R=\frac{\Delta R}{R}=\frac{R_{A P}-R_{P}}{R_{P}},
$$




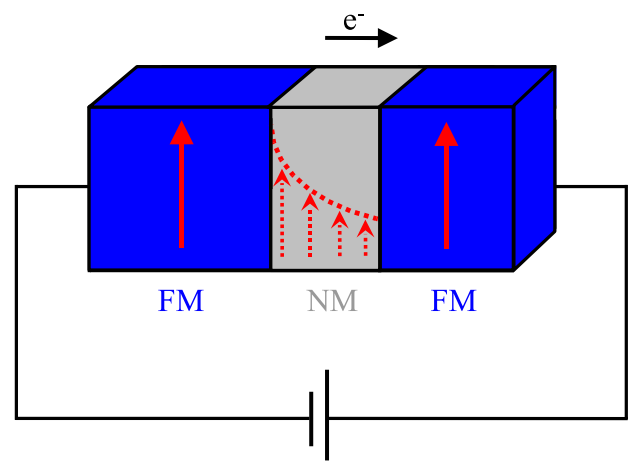

Figure 2.7: Schematic illustration of a simple trilayer GMR structure.

where $R_{P}$ and $R_{A P}$ represent the resistance of the GMR structure in the parallel and the antiparallel configuration, respectively. ${ }^{1}$

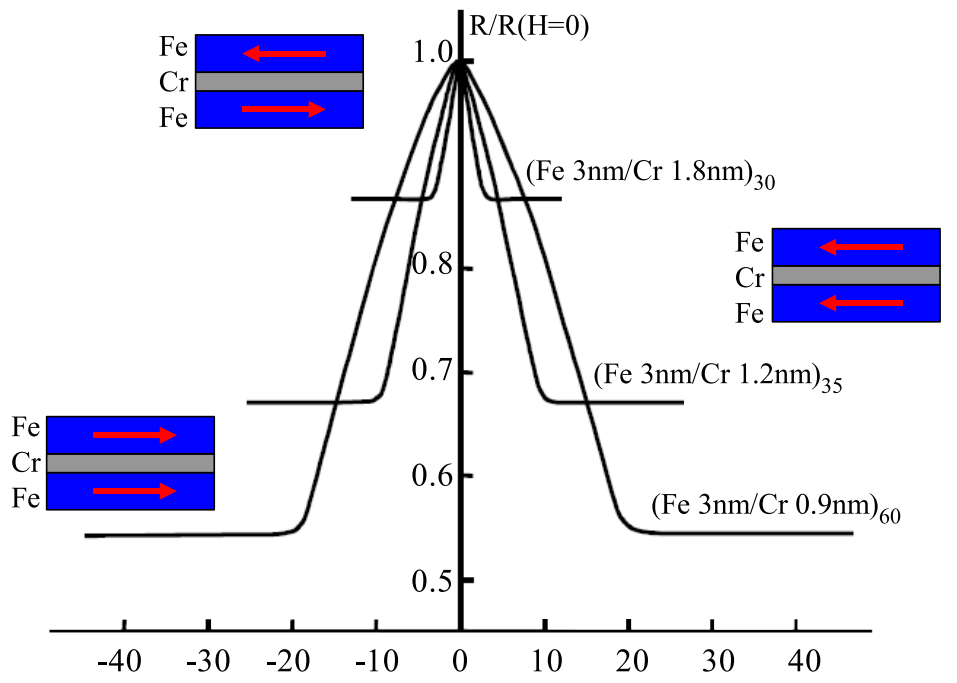

Magnetic field $[\mathrm{kG}]$

Figure 2.8: Experimental illustration of a simple GMR structure. The colored insets show the relative orientation of the magnetization in the two ferromagnetic layers at the corresponding external field values (after [30]).

A crucial point is the initial antiparallel alignment of the ferromagnets. There are several possibilities to provide this magnetic configuration:

- the first option relies on the effect of antiferromagnetic interlayer exchange coupling. Here the Ruderman-Kittel-Kasuya-Yoshida (RKKY) coupling between localized moments via the conduction electrons of

\footnotetext{
${ }^{1}$ Since in most papers a definition in which the GMR is normalized to the low resistance value $R_{P}$ can be found, this notation is also used throughout this thesis.
} 
the non-magnetic spacer layer forces the two ferromagnets to be magnetically coupled. This coupling mechanism oscillates as a function of spacer thickness between ferromagnetic and antiferromagnetic. Selecting an appropriate thickness thus guarantees an antiparallel configuration of the two ferromagnetic layers in low applied fields, and an alignment occurs only in relatively high external fields. The $\mathrm{Fe} / \mathrm{Cr}$ samples presented in figure 2.8 are a standard example for an interlayer exchange coupled system.

- In a spin valve configuration one of the ferromagnetic layers is pinned due to exchange coupling with a directly adjacent antiferromagnetic layer, while the other ferromagnet can still rotate under the influence of the external magnetic field.

- A third possibility is to choose ferromagnetic layers with different coercitivities. Thus in a low external field the soft magnetic layer switches, whereas the hard magnetic layer remains in the previous direction. It only rotates in much higher applied fields, so that a wide field range with antiparallel alignment of the two layers exists. For the GMR structures produced within this thesis work the different coercitivity fields are realized by dissimilar ferromagnetic layer thicknesses.

\subsubsection{Current in Plane and Current Perpendicular to Plane GMR}

There are two configurations in which the GMR effect can be observed: they are referred to as the current in plane (CIP) and the current perpendicular to plane (CPP) geometry as depicted in figure 2.9. Both GMR types are characterized by a drop in sample resistance in higher magnetic fields as described above, but the relevant physics and the corresponding models are rather different.

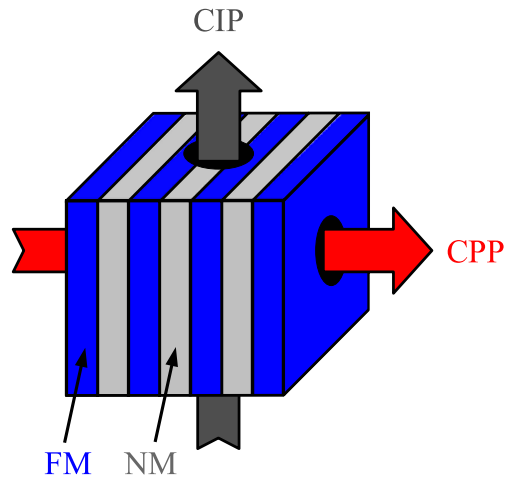

Figure 2.9: Current in plane (CIP) and current perpendicular to plane $(C P P)$ geometries for a multilayer GMR stack.

This is a consequence of the different length scales involved. In the CPP geometry, due to spin accumulation effects, the thickness of the nonmagnetic spacer layer has to be smaller than the spin diffusion length for the 
same material - otherwise no GMR will be observed. A very simple model explaining the CPP GMR by an analogy with the phenomenon of polarized light is presented in the following section. Afterwards the GMR effect is discussed in terms of spin-dependent scattering of conduction electrons, and a corresponding resistor model is presented. Also the treatment of CPP GMR within the Valet-Fert theory will be shortly discussed (section 2.6.4).

In the CIP geometry fundamentally different symmetry considerations show that no spin accumulation arises in this case. The transport can still be described by spin-dependent scattering events, but these predominantly occur in the ferromagnetic layers or at their interfaces and not in the nonmagnetic spacer layer itself. Since the current flows along the layers, an electron will leave its present layer and drift through different magnetic layers only if the mean free path is long compared to the thickness of the non-magnetic spacer layer. Thus the characteristic length scale in the CIP configuration is given by the mean free path of the conduction electrons. The CIP GMR has not been studied within this thesis; hence, in the following text the term GMR will always refer to the CPP geometry.

\subsubsection{An Analogy with Polarized Light: Spin-Dependent Transmissions}

Assuming that the first ferromagnet of the GMR structure emits a current which is $100 \%$ spin polarized (i.e. it is an ideal half-metallic ferromagnet), it can be compared to an optical polarizer which passes only one polarization component of light [31]. In this way a spin accumulation arises in the non-magnetic interlayer. Provided that the thickness of this spacer layer is much smaller than the spin diffusion length, the spin accumulation reaches across the normal metal to the second ferromagnetic layer. Being also halfmetallic, this second ferromagnet acts as a spin filter - just like an optical analyzer. This means, that only one spin species can pass through the second ferromagnet, while the other one is being reflected at its interface with the normal metal. Depending on the relative orientation of the spin accumulation with the magnetization of the second ferromagnet, the entire current is either transmitted through the device (parallel alignment of the two ferromagnets) or totally blocked (antiparallel alignment of the two ferromagnets). The central difference with the case of crossed optical polarizers is that in optics the extinction angle is $90^{\circ}$, while for the magnetizations of the GMR structure it is $180^{\circ}$.

\subsubsection{Microscopic Picture: Spin-Dependent Scattering and the Two Resistor Model of GMR}

Making use of the Mott model as presented in section 2.3, it is straightforward to explain the GMR. As it is depicted in figure 2.10, two collinear magnetization directions (parallel and antiparallel) of two successive ferromagnetic layers are considered in the CPP geometry. According to Mott there is a strong asymmetry in the density of states at the Fermi level giving 
rise to different numbers of final states, which is here reflected in different scattering probabilities for the two spin species: scattering is strong for electrons with spin antiparallel to the magnetization direction, while it is weak for electrons with spin parallel to the magnetization of the ferromagnet. Hence, for parallel alignment of the two ferromagnets (top panel of figure 2.10 (a)) there is hardly any scattering for spin-up electrons, but spin-down electrons are heavily scattered due to their spin direction being antiparallel to the magnetization of both layers. In the case of antiparallel alignment (top panel of figure 2.10 (b)) both spin species are scattered strongly in one of the ferromagnetic layers, namely in the one in which their spin is antiparallel to the magnetization.

(a)
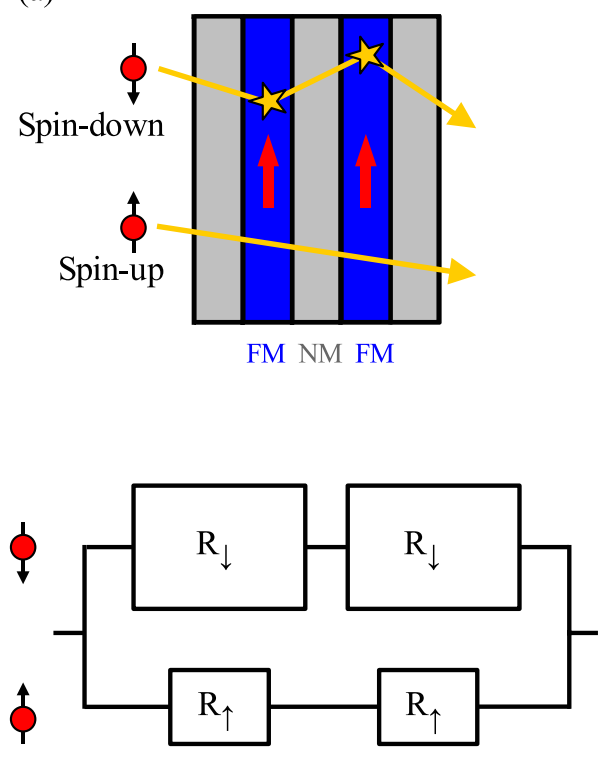

(b)
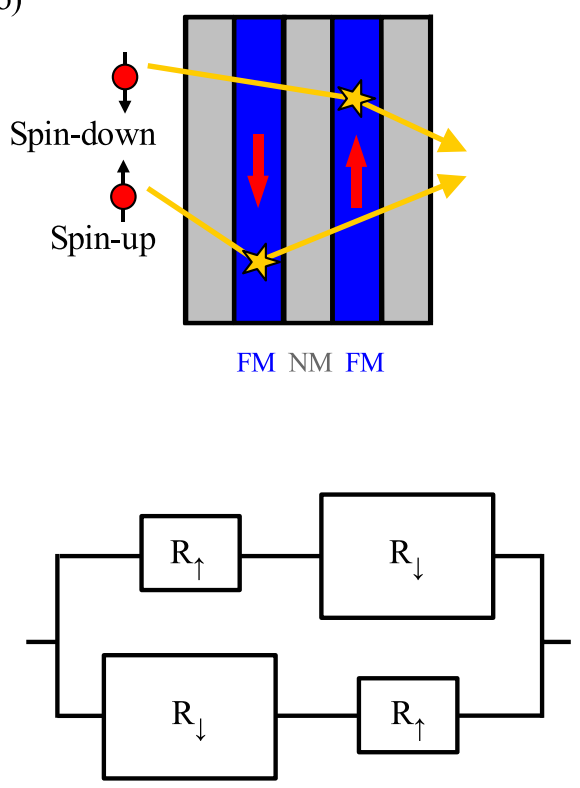

Figure 2.10: Simplified picture of electron transport in a GMR stack for parallel (a) and antiparallel (b) magnetizations of two successive ferromagnetic layers. The yellow lines represent individual electron trajectories within the two spin channels; scattering events are indicated by yellow stars. The bottom panels show the corresponding resistor networks within the two-current series resistor model.

A simple resistor network analogy is appropriate to explain the resulting different resistivities for both configurations. The resistivities of each layer for each spin direction are added in series, while those for two spin channels (i.e. the majority and the minority spin channel) are added in parallel. From this it follows that there is a sort of short-circuit effect in the parallel configuration due to the lack of scattering events for spin-up electrons. Thus, the total resistivity of the multilayer is lower for parallel than for antiparallel alignment of the magnetizations. 

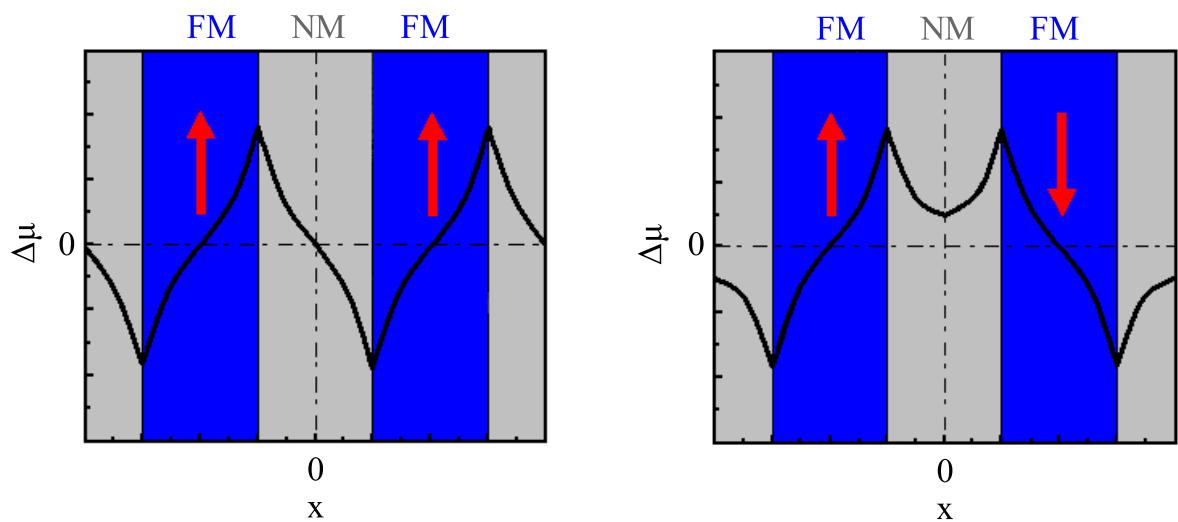

Figure 2.11: Spin accumulation $\Delta \mu=\mu_{\uparrow}-\mu_{\downarrow}$ in a multilayer $G M R$ stack for parallel (left) and antiparallel (right) alignment. The curves were obtained by using the general solutions for a homogeneous layer given by equation 2.13 and taking into account the proper boundary conditions at each interface. From [22].

\subsubsection{The Valet-Fert Interpretation of GMR}

The Valet-Fert model described in section 2.5.2 for a ferromagnetic/nonmagnetic interface can be extended to a trilayer GMR stack. This implies writing the one dimensional solution of equation 2.13 as the sum of forward and backward decaying exponentials for each layer and then matching the electrochemical potentials and spin currents at the interfaces for each spin channel. Figure 2.11 shows the corresponding curves for the difference of the spin-dependent electrochemical potentials (i.e. for the spin accumulation) as they were calculated by Valet and Fert in [22]. Here only bulk spin-dependent scattering was taken into account, whereas interface spindependent scattering was neglected. This is maintainable only for structures with layer thicknesses of a few hundred Ångströms or less, since otherwise the magnitude of the interface contributions is expected to exceed the bulk contributions [22]. Hence, in contrast to figure 2.6 (section 2.5.2), no true discontinuities of the potential at the interfaces can be seen in the plots of figure 2.11 due to the lack of spin flip processes.

Nonetheless, it can be observed that the effective resistance of the nonmagnetic spacer layer varies with the magnetic configuration, which results in magnetically dependent changes in the resistivity of the entire layer stack, i.e. GMR.

\subsection{Spin Injection Into Ferromagnets: Spin Torque and Dynamics}

The discussion of the GMR effect revealed that the relative orientation of the magnetization of two ferromagnetic layers affects the electric current passing 
through this device, causing different resistances for different magnetic configurations. The reverse effect, that a spin-polarized current can influence the magnetic moment of a ferromagnetic layer is the central topic of the experiments presented in this thesis. In the CPP geometry, spin-polarized currents may transfer angular momentum between the ferromagnets, resulting in current-induced excitations in the magnetic layers.

\subsubsection{Phenomenological Description}

In the following the basics of current-induced switching are introduced exemplarily by describing pioneering experiments by Katine et al. [32] as they are displayed in figure 2.12. The sample is a GMR trilayer, consisting of a
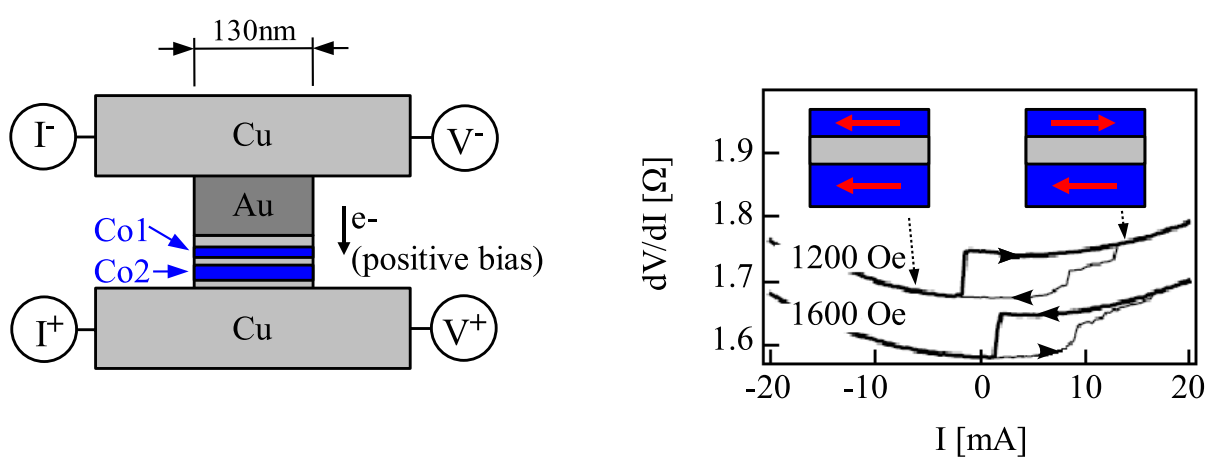

Figure 2.12: Pioneering current-induced switching experiments performed by Katine et al. [32]: GMR trilayer geometry (left) and corresponding graphs displaying the differential resistance $d V / d I$ as a function of current I taken at room temperature with magnetic fields applied in the plane of the film.

first 10nm thick Co layer, which is separated by a $\mathrm{Cu}$ spacer layer of $6 \mathrm{~nm}$ thickness from the second Co layer. The latter one is only $2.5 \mathrm{~nm}$ thick, and due to its comparatively low coercitivity it is referred to as the 'free' layer, while the first Co layer is 'fixed' by the external magnetic in-plane field of 12000 . The pillar geometry restricts the lateral dimensions to approximately $130 \mathrm{~nm}$. Thus relatively high current densities in the region of $10^{7}-10^{8} \mathrm{~A} / \mathrm{cm}^{2}$ can be passed through the structure, and simultaneously the differential resistance $d V / d I$ across the contact is measured in a four point geometry in order to probe its GMR state. As the right panel of figure 2.12 shows, the parallel alignment (low resistivity) of the two Co layers is stabilized at negative current bias, which means that the electrons flow from the fixed to the free layer. However, at positive current bias the alignment is destabilized, and at sufficiently large currents the free Co layer switches to an antiparallel configuration (high resistivity). Sweeping the current back towards negative values reveals that parallel alignment is now reached again at smaller currents, i.e. the switching behavior is hysteretic. Increasing the external field to 16000 e (lower curve in figure 2.12) shifts the currents required for switching to higher values, because the Zeeman 
energy of the applied field clearly favors parallel alignment. Besides, at zero bias current the high resistance state can still be stable, and the antiparallel configuration is only retrieved for negative currents.

This asymmetry in current of the observed switching clearly indicates that the cause is the spin transfer effect rather than the Oersted field. Any changes in the magnetic configuration due to the Oersted field alone are expected to be symmetric in current, whereas the spin transfer torque is explicitly asymmetric [33].

\subsubsection{Physical Picture: Absorption of the Transverse Spin Current Component}

When a spin-polarized current is injected from a non-magnet through a perfect interface into a ferromagnet, a spin transfer torque arises due to the transfer of spin angular momentum from the conduction electrons to the background magnetization of the ferromagnet. The reason for this angular momentum transfer is the absorption of the transverse component of the spin current by the interface. A comprehensive theoretical discussion on these effects can be found in [34]; here only the main results of reference [34] are summarized following the presentation given in [35].

(a)

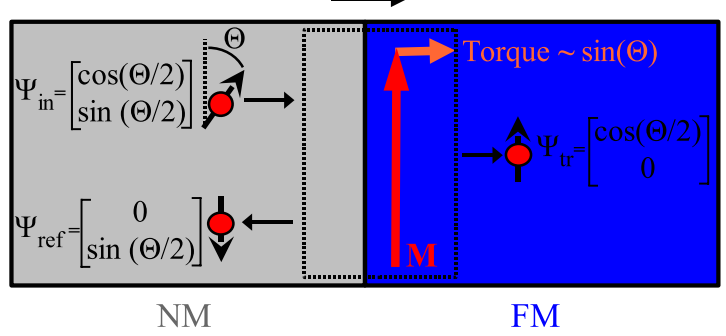

(b)

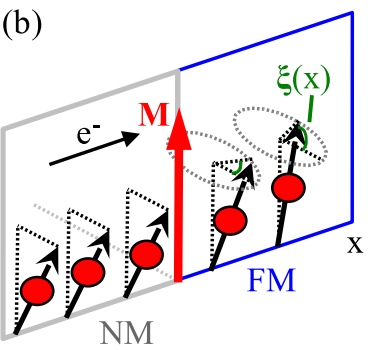

Figure 2.13: Two out of three effects contributing to the absorption of the transverse component of spin current at the interface between a non-magnet and a ferromagnet: (a) spin filtering and (b) spatial precession of the spins in the ferromagnet. Adapted from [35].

There are three distinct mechanisms which contribute to the absorption of transverse spin current:

- spin-dependent reflection and transmission (spin-filtering)

- rotation of transmitted and reflected spins

- spatial precession of the spins in the ferromagnet.

The first process becomes clearer upon considering figure 2.13 (a). The current impinging on the interface is spin-polarized along an axis which is tilted at an angle $\Theta$ relative to the magnetization axis in the ferromagnet. Here the polarization axis is chosen to lie in the drawing plane in order to 
reduce the problem to two dimensions, whereas in real experiments (due to the shape anisotropy) the polarization axis is usually expected to be in the plane of the layers. The normalized wave function $\Psi_{\text {in }}$ of an incident electron with a non-zero spin component transverse to the magnetization in the ferromagnetic layer can now be written as a linear combination of spin-up and spin-down components with respect to the quantization axis defined by the magnetization. The amplitudes of $\cos (\Theta / 2)$ and $\sin (\Theta / 2)$ each correspond to a specific transverse component of the spin vector given by $\sin (\Theta)$. Due to the spin asymmetry and the resulting spin-dependent transmission of electrons at the interface (cf. section 2.3), the incident, the transmitted and the reflected wavefunctions $\left(\Psi_{\mathrm{in}}, \Psi_{\mathrm{tr}}\right.$ and $\left.\Psi_{\text {ref }}\right)$ differ from each other. These differences lead to a discontinuity in the transverse spin current. Since the angular momentum still has to be preserved, the transverse component of the spin current is absorbed by the interface, which results in a current-induced torque on the magnetization of the ferromagnet indicated by the orange arrow in figure 2.13. Here the ideal case of perfect spin-filtering is displayed, while approximately only $50 \%$ of the transversal component is absorbed in realistic scenarios.

The second mechanism which also reduces the transverse spin components of $\Psi_{\text {tr }}$ and $\Psi_{\text {ref }}$ occurs when summing over the entire ensemble of conduction electrons. Because of their complex reflection and transmission amplitudes the spin of electrons generally rotates upon contact with the interface. This rotation is non-classical and its amount considerably differs for electrons with wave vectors from different Fermi energies. However, phase cancellation is achieved when summing over all electrons. Hence, very little of the reflected transverse spin component remains, while the reduction of the transmitted transverse spin current is less significant.

Finally, the third effect of spatial precession of the spins in the ferromagnet as depicted in figure 2.13 (b) has to be taken into account. As a consequence of the spin-split density of states the transmitted electrons in the ferromagnet possess spin-up and spin-down components of the same total energy $E_{F}$, but different kinetic energy and thus different wave vectors: $k^{\downarrow}-k^{\uparrow} \neq 0$. These two states are coherent, so that a spatial phase $\xi(x)=\xi_{0}+\left(k^{\downarrow}-k^{\uparrow}\right) \cdot x$ emerges (cf. figure $2.13(\mathrm{~b})$ ). This means that the spins precess in space as they propagate away from the interface, where the spatial precession frequency varies noticeably for electrons from different portions of the Fermi surface. Thus summing over all conduction electrons leads to a reduction of the transverse spin component: the net result is a precessing spin current which damps out completely after a few lattice constants.

The calculations performed in [34] reveal that the relative importance of the three effects described above varies for different materials as well as for different crystallographic orientations. Nonetheless, the final outcome is always the same: the transverse component of the spin current essentially disappears at the interface, so that the related transfer of angular momentum produces a torque on the magnetization directly at the interface.

The microscopic picture of current-induced excitations developed in this 
(a)

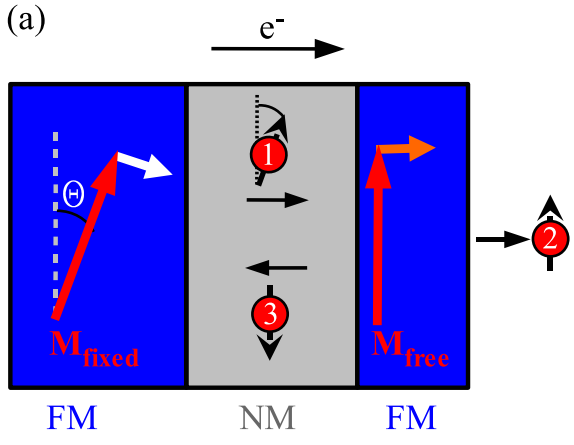

(b)

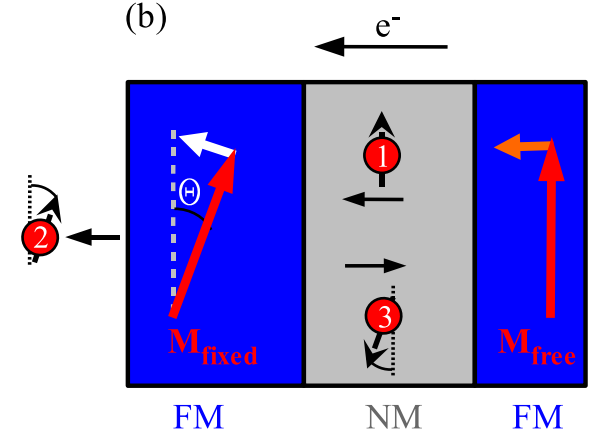

Figure 2.14: Schematic illustration of the mechanism of current-induced switching. Due to the different coercitivities of the magnetic layers only the free layer responds to the spin torque effect by means of a reorientation (orange arrows), while the magnetization in the fixed layer remains stable (white arrows). (a) and (b) display the scenarios for opposite current directions, which result in parallel and antiparallel alignment of the magnetizations in both layers, respectively. Adapted from [35].

section will now be rounded off by looking at the mechanisms in a complete trilayer GMR structure as shown in figure 2.14 (similar to the structures discussed in sections 2.6 and 2.7.1). In the left panel the current flows from the fixed layer, which is acting as a spin polarizer, to the free layer (1). Here it transfers its transverse spin component in the form of a torque to the free layer. Part of the current is transmitted (2), while the rest is reflected (3). The reflected spin current returns to the fixed layer, where its transverse component is again absorbed and a torque on the fixed layer arises. Because of the higher coercitivity of the fixed magnetic layer, the torque is not sufficient to influence the magnetization direction here. Hence, only the free layer will begin to rotate in order to reach a stable parallel alignment with the fixed one. In the case of opposite current bias (figure 2.14 (b)) the situation is very similar to the one depicted in (a), but both torques point in the opposite direction. Again, only the magnetization in the free layer will react and start to rotate. If the torque is sufficiently large, i.e. the current density is high enough, the free layer will reverse completely to the antiparallel configuration. This will result in a higher resistance of the entire structure as discussed in the previous section. Also if this state of total antiparallel alignment is not reached, a change in resistance can possibly still be detected when the magnetic moment of the affected layer is set into rotation - for example in high external fields which can inhibit a complete reversal [36]. 


\subsubsection{Extending the Landau-Lifshitz-Gilbert Equation: The Effective Field and the Spin Torque Transfer Term}

The motion of the magnetization $\vec{M}$ in an effective external magnetic field $\vec{H}_{\text {eff }}$ is described by the Landau-Lifshitz-Gilbert equation

$$
\frac{d}{d t} \vec{M}=-\gamma_{0} \vec{M} \times \vec{H}_{e f f}+\frac{\alpha}{M_{S}}\left(\vec{M} \times \frac{d}{d t} \vec{M}\right) .
$$

The term with the gyromagnetic ratio $\gamma_{0}$ indicates a precessional motion of the magnetization (figure 2.15 (a)), while the second addend is a phenomenological damping term with the Gilbert damping constant $\alpha$ and the saturation magnetization $M_{S}$. So the latter term signifies an additional motion, which is directed towards the low energy equilibrium state of the system (figure 2.15 (b)). For a complete derivation and discussion of equation 2.17 the reader is referred to [37].

Here the influence of the spin torque effect on the magnetization dynamics and the corresponding modifications of the Landau-Lifshitz-Gilbert equation are discussed. The description is based on the calculations performed in $[23,38,39]$.

In a first step the angular momentum transfer between the spin-polarized current and background magnetization has to be taken into account. For this reason the Hamiltonian operator $H_{i n t}$, which describes the so-called sd interaction between the spin accumulation $\vec{m}$ and the localized moments of the ferromagnetic layer $\vec{M}_{d}$, is introduced:

$$
H_{\text {int }}=-J \vec{m} \cdot \vec{M}_{d}
$$

Here $\vec{M}_{d}$ denotes the unit vector in the direction of the local magnetization $\vec{M}$, and $J$ is the exchange coupling parameter. This coupling mechanism alters the continuity equation for the spin accumulation 2.9 (here presented with vector quantities) in the following way:

$$
\frac{\partial \vec{m}}{\partial t}+\nabla \cdot \vec{J}_{m}+\frac{J}{\hbar} \vec{m} \times \vec{M}_{d}=-\frac{2 \vec{m}}{\tau_{s f}} .
$$

The new third term on the left hand side describes a precession of the injected spin due to the sd interaction if the magnetization directions of the spin accumulation and the local moments are not perfectly aligned. However, according to Newton's third law (action=reaction) also the opposite effect, namely the impact of the spin accumulation on the background magnetization has to be considered. This is accounted for by including the spin accumulation as an additional field term $J \vec{m}$ into the Landau-LifshitzGilbert equation for the local magnetization $\vec{M}_{d}$ :

$$
\frac{d \vec{M}_{d}}{d t}=-\gamma_{0} \vec{M}_{d} \times\left(\vec{H}_{e f f}+J \vec{m}\right)+\frac{\alpha}{M_{S}}\left(\vec{M}_{d} \times \frac{d \vec{M}_{d}}{d t}\right) .
$$

This equation cannot be solved independently from equation 2.19, because the dynamics of the spin accumulation throughout the device is directly 
related to the magnetization dynamics of the local moment. However, this problem can be circumvented by comparing the relevant timescales of both processes involved: while the spin accumulation is determined by the spinflip relaxation time and $J / \hbar$, which correspond to the picosecond time scale, the local magnetization in lower magnetic fields only evolves on the nanosecond time scale [39]. For this reason the spin accumulation can be treated in the stationary limit, i.e. the local moments are assumed to be independent of time on the nanosecond scale, and hence equation 2.19 is reduced to

$$
\nabla \cdot \vec{J}_{m}+\frac{J}{\hbar} \vec{m} \times \vec{M}_{d}=-\frac{2 \vec{m}}{\tau_{s f}} .
$$

For determining the steady state solutions of this equation, it is further simplified by splitting up the spin accumulation $\vec{m}$ into one component $\vec{m}_{\|}$ parallel to the local magnetization and a second component $\vec{m}_{\perp}$ transverse to the local magnetization [23]. This yields the following two relations:

$$
\begin{gathered}
\frac{\partial^{2} \vec{m}_{\|}}{\partial x^{2}}-\frac{\vec{m}_{\|}}{\lambda_{s d}^{2}}=0, \\
\frac{\partial^{2} \vec{m}_{\perp}}{\partial x^{2}}-\frac{\vec{m}_{\perp}}{\lambda_{s f}^{2}}-\frac{\vec{m}_{\perp} \times \vec{M}_{d}}{\lambda_{J}^{2}}=0,
\end{gathered}
$$

where $\lambda_{J}$ represents the decay of transverse spin currents due to spindependent scattering without spin-flip processes given by $\lambda_{J}=\sqrt{\hbar D / J}$. Having a close look at the different relevant length scales reveals that the longitudinal spin accumulation $m_{\|}$decays at the length scale of the spin diffusion length, which corresponds to approximately 60nm in Co [36]. The transverse spin accumulation $m_{\perp}$ on the other hand is governed by the decay of $\lambda_{J}$ (if $\lambda_{J} \ll \lambda_{s f}$ ), which amounts to only 1.5-3nm [39].

From the equations 2.22 and 2.23 it can be concluded that the longitudinal spin accumulation does not influence the local magnetic moment $\vec{M}_{d}$ in relation 2.20. Thus the latter equation is modified to

$$
\frac{d \vec{M}_{d}}{d t}=-\gamma_{0} \vec{M}_{d} \times\left(\vec{H}_{e f f}+J \vec{m}_{\perp}\right)+\frac{\alpha}{M_{S}}\left(\vec{M}_{d} \times \frac{d \vec{M}_{d}}{d t}\right) .
$$

In order to simplify the problem further it is reasonable to distinguish between the magnetization of the layer with a high coercitivity $\vec{M}_{d}^{\text {fixed }}$ (acting as a polarizer) and the magnetization of the layer with the lower coercitivity $\vec{M}_{d} \equiv \vec{M}_{d}^{\text {free }}$ (being free to rotate and switch). As it is demonstrated in reference [38], the two components of the spin accumulation in the plane transverse to $\vec{M}_{d}^{\text {free }}$ can thus be written as

$$
\begin{aligned}
J \vec{m}_{\perp} & =a \vec{M}_{d}^{\text {fixed }} \times \vec{M}_{d}^{\text {free }}+b\left(\vec{M}_{d}^{\text {free }} \times \vec{M}_{d}^{\text {fixed }}\right) \times \vec{M}_{d}^{\text {free }} \\
& =a \vec{M}_{d}^{\text {fixed }} \times \vec{M}_{d}^{\text {free }}+b \vec{M}_{d}^{\text {fixed }}
\end{aligned}
$$

where $a$ and $b$ are constants proportional to the current density, whose exact values are determined by some further geometric details of the device. Inserting relation 2.25 in the modified equation of motion 2.24 leads to 

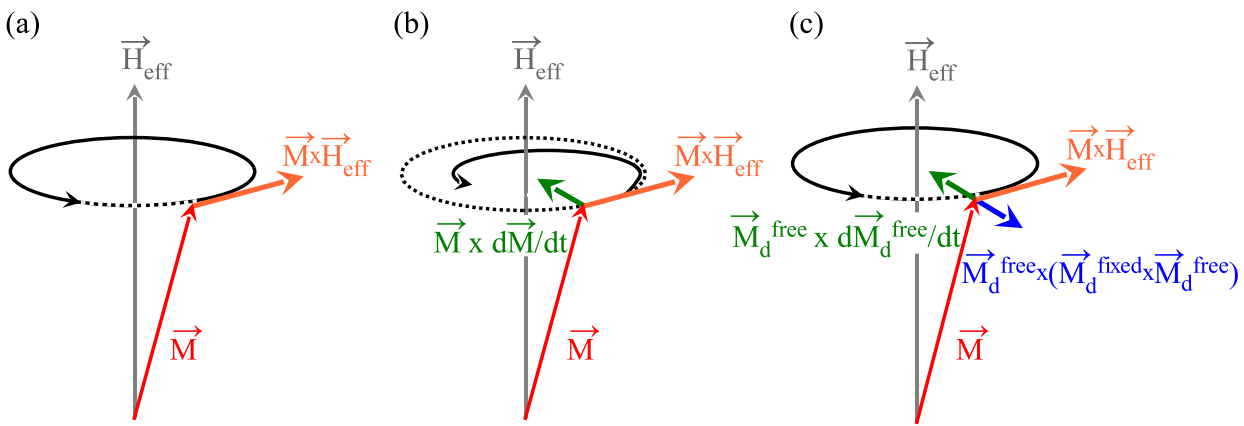

Figure 2.15: Illustration of the Landau-Lifshitz-Gilbert equation: (a) precessional motion without damping (b) including the Gilbert damping term (c) extended equation with the spin transfer torque opposing the damping.

$$
\begin{aligned}
\frac{d}{d t} \vec{M}_{d}^{\text {free }}= & -\gamma_{0} \vec{M}_{d}^{\text {free }} \times\left(\vec{H}_{\text {eff }}+b \vec{M}_{d}^{\text {fixed }}\right)-\gamma_{0} a \vec{M}_{d}^{\text {free }} \times \\
& \left(\vec{M}_{d}^{\text {fixed }} \times \vec{M}_{d}^{\text {free }}\right)+\frac{\alpha}{M_{S}}\left(\vec{M}_{d}^{\text {free }} \times \frac{d}{d t} \vec{M}_{d}^{\text {free }}\right) .
\end{aligned}
$$

This is the final form of the extended Landau-Lifshitz-Gilbert equation. It comprises an additional effective field term

$$
b \vec{M}_{d}^{\text {fixed }} \text {, }
$$

which influences the precession frequency of the magnetization. Besides, relation 2.26 features an extra torque term

$$
\gamma_{0} \vec{M}_{d}^{\text {free }} \times\left(\vec{M}_{d}^{\text {fixed }} \times \vec{M}_{d}^{\text {free }}\right),
$$

the so-called spin torque transfer term. This looks very similar to the Gilbert damping term, but depending on the direction of current, it can either be negative or positive. In the latter case it can possibly overcome the damping (figure 2.15 (c)) and enhance the precession amplitude, which corresponds to the initiation of current-induced excitations.

\subsubsection{The Critical Current Density}

In the previous sections it has already been pointed out that there is a threshold for current-induced excitations, which is determined by the competition between the spin-transfer torque and magnetic damping. Starting from the extended Landau-Lifshitz-Gilbert equation 2.24, the critical current required for magnetization reversal can be estimated by a stability analysis [2, 40]. For applied fields $H_{\text {ext }}$ and switching processes from the parallel to the antiparallel configuration $(\Theta=0)$ and from the antiparallel to the parallel configuration $(\Theta=\pi)$, respectively, this yields [40, 41]:

$$
\begin{aligned}
I_{C}^{\Theta=0} & \approx \frac{A \alpha M_{S} V}{g(\Theta=0) p}\left(H_{e x t}+H_{K}\right) \\
I_{C}^{\Theta=\pi} & \approx \frac{A \alpha M_{S} V}{g(\Theta=\pi) p}\left(H_{e x t}-H_{K}\right) .
\end{aligned}
$$


Here $M_{S}, V$ and $\alpha$ denote the saturation magnetization, the volume and the Gilbert damping parameter for the free layer, respectively. The constant $g$ depends on the relative angle $\Theta$ between the magnetization direction of the fixed layer and the one of the free layer, where the specific angular dependence is determined by the factor $p$ [40]. The factor $A$ is dependent on the specifics of the transport model, but it roughly amounts to $3 \cdot 10^{11} \mathrm{~mA} \mathrm{Oe}^{-1}$ $\mathrm{emu}^{-1}[41] . H_{K}$ represents the effective uniaxial anisotropy field coefficient including effects of both magnetocrystalline and shape anisotropy.

Plugging in typical values in equations 2.29 and 2.30 results in critical current densities of about $10^{7}-10^{8} \mathrm{~A} / \mathrm{cm}^{2}$ for trilayer GMR structures. This order of magnitude is also confirmed by many experimental studies. In order to accomplish such high current densities, small structures sizes of approximately $100 \mathrm{~nm}$ in diameter have to be prepared due to total power limitations. At the same time these sample dimensions guarantee that the spin transfer effect prevails over the effect of the Oersted-Maxwell field: Since the Oersted-Maxwell field varies as $H_{O M} \propto I / r$, where $r$ is the radius of the nanostructure, and the spin-transfer torque can be viewed to create an effective exchange field scaling as $H_{e x} \propto I / r^{2}$, the ratio $H_{e x} / H_{O M}$ favors exchange over Oersted-Maxwell fields at nanoscale values of $r$.

\subsection{Current-Induced Excitations in Ferromagnetic Single Layers}

So far only the spin-transfer effect in metallic trilayer junctions with a thick ferromagnetic polarizing layer and another thinner ferromagnetic layer acting as a spin analyzer has been discussed. However, in quite recent theoretical studies it was shown that current-induced excitations can even emerge in thin ferromagnetic single layers sandwiched between two normal metals $[42,43]$, i.e. without any prior spin polarization of the electrical current. These results were at least qualitatively confirmed in point contact experiments performed by $[7,8]$ as well as in first studies on single layer nanopillars by Özyilmaz et al. [9, 20].

In this section the key mechanisms behind the observed current-induced excitations in single layers will be explained from a rather phenomenological point of view, summarizing the most important theoretical findings. For a more detailed quantitative description of spin wave instabilities the reader is referred to $[42,43,44]$, where the authors perform a linear stability analysis in order to predict the onset of current-induced excitations. While Polianski and Brouwer [42] study the case of a homogeneously magnetized ferromagnet parallel to the current, Stiles et al. [43] have relaxed this requirement and allow for the magnetization to vary along the direction of current flow. The dynamics of the spin waves beyond the instabilities are further investigated in [45], where an ansatz from perturbation theory is used in the smallamplitude regime and numerical solutions for the magnetization dynamics are derived for even higher current densities and larger amplitudes.

There are three effects which all play a central role in the spin torque 
effect for single ferromagnetic layers:

- spin filtering

- spin diffusion along the interface

- fluctuations in the magnetization of the ferromagnet.

This is further elucidated by means of figure 2.16. In its left panel (a) the electrons flow from a non-magnetic metal towards the ferromagnetic layer. Due to the different scattering probabilities for the two spin species, some spins are reflected back into the non-magnet directly at the interface (minority spins). These backscattered electrons are spin-polarized with a polarization direction directly antiparallel to the direction of the local magnetization of the ferromagnetic layer. As explained in the previous section, the electrons will transfer the perpendicular component of their spin to the ferromagnet, hence each exerting a torque. But since the current is not spin-polarized, the different torque directions of all electrons impinging for the first time will cancel each other out and thus no net torque is passed on. Since the motion of the electrons is diffusive, there is a significant probability that the scattered electrons return to the interface at a slightly different place [33]. In the case of a totally homogeneous magnetization of the ferromagnet, the spins are antiparallel to the local magnetization and no further transfer of angular momentum will occur. Hence, in the situation of figure 2.16 (a) only a spin accumulation which is on average antiparallel to the magnetization of the ferromagnet will emerge in the normal metal.

(a)

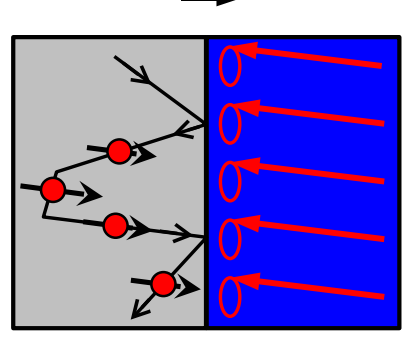

NM (b)

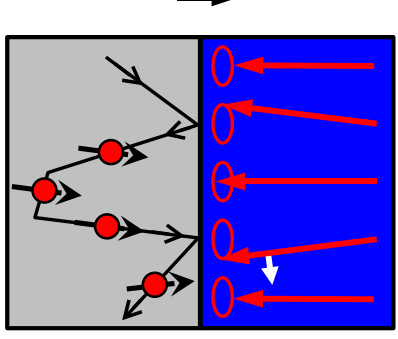

FM (c)

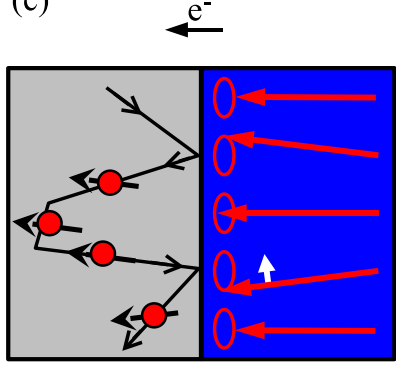

FM

Figure 2.16: Spin transfer torque: (a) no torque due to the lateral homogeneity of the magnetization (b) destabilizing torque for positive current bias (c) stabilizing torque for negative current bias. Adapted from [33].

This will change if the magnetization of the ferromagnetic layer perpendicular to the direction of current flow slowly varies as depicted in figure 2.16 (b). Here the electrons are still antiparallel to the local magnetization after their first reflection, but when they diffuse laterally, this is no longer the case for the rescattering event: thus the magnetization exerts a torque on the spins and in return they also exert a reaction torque on the local moments of the ferromagnet. These mechanisms tend to amplify the spin wave, 
because in this situation the diffusing spins are on average antiparallel to the magnetization (minority spin accumulation as already described above for (a)).

The right panel of figure 2.16 shows the corresponding drawing for the opposite current direction, i.e. the electrons flow from the ferromagnet into the normal metal. In this case the diffusing spins are on average parallel to the magnetization (majority spin accumulation), which means that the torque which is transferred upon scattering from the interface now tends to suppress fluctuations away from the average magnetization direction. In this way the spin wave amplitude is reduced.

Combining the situations sketched in figure 2.16 (b) and (c) reveals that for a thin ferromagnetic layer sandwiched between two identical normal metal electrodes the resulting torques at both interfaces are of equal magnitude but opposite direction. Hence, they cancel each other out, and no current-induced instabilities in the magnetization of the single layer film occur. This has also been demonstrated experimentally in [9]. However, the mirror symmetry can be broken either by inserting asymmetric contacts or by a spatial variation of the magnetization in the ferromagnet along the current direction. In both cases the torques acting on each nonmagnetic/ferromagnetic interface have different magnitudes, so that for sufficient current densities spin wave instabilities can appear.

(a)

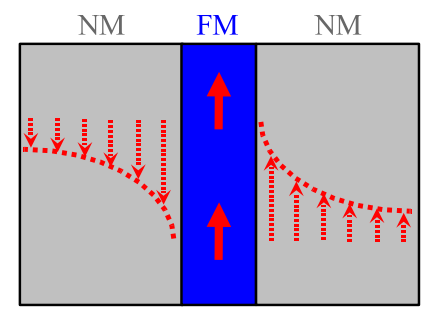

(b)

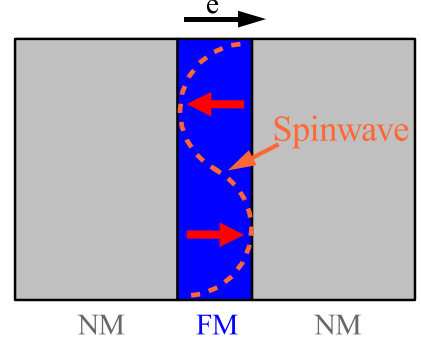

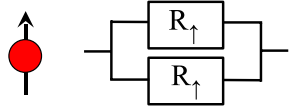
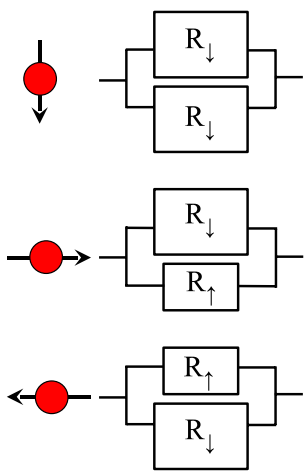

Figure 2.17: Simple resistor model for a ferromagnetic single layer with a spatially uniform magnetization (a) and with a spin wave excitation (b). $R_{\uparrow}$ and $R_{\downarrow}$ refer to the majority and minority resistances, respectively. Adapted from [45].

The effect of these excitations on the device resistance can be estimated by some simple considerations as they are illustrated in figure 2.17. The panel on the upper left (a) shows the spin accumulation on both sides of the ferromagnet for a layer with symmetric leads as well as a totally uniform magnetization. The corresponding circuit diagram on its right depicts the 
resistivities seen by the two spin species, where the large and the small resistor symbols ( $R_{\downarrow}$ and $R_{\uparrow}$ ) refer to the minority and majority resistivities, respectively. Once a spin wave has been established, the non-uniformity of the magnetization reduces the amount of spin accumulation in the normal metal. This results in a decrease of the entire device resistance as figure 2.17 (b) points out: the ferromagnet with the current-induced spin wave is here shown as a large angle excitation simplified to a parallel configuration of ferromagnets with opposite magnetization directions. The related circuit diagram visualizes the lower net resistance in comparison to the situation shown in (a). Indeed, the experiments performed by Özyilmaz et al. demonstrated, that such a decrease in resistance is one of the anomalies observed in the $I(V)$ curves of asymmetric, single ferromagnetic Co nanopillars. 


\section{CHAPTER 3}

\section{Experimental Equipment and Techniques}

As an experimental thesis it is appropriate to give an overview of the various techniques used during the fabrication and investigation of the ferromagnetic nanostructures. Since the main focus has been to set up a complete fabrication process for magnetic nanopillars, the methods involved in this process (construction of a UHV chamber, nanopatterning by e-beam lithography, e-beam evaporation) will take center stage.

\subsection{Sample Preparation}

\subsubsection{Nanofabrication Technique: Electron Beam Lithography}

Electron beam lithography (EBL) is a widely used patterning method in research and mask fabrication industry with a sub-100nm resolution. The technique in brief consists of a scanning electron microscope (SEM), which scans an electron beam across a sample coated with a resist film sensitive to those electrons, thus depositing energy in the desired pattern in the resist film. After this exposure process the modified areas of the resist film can be dissolved preferentially by a developer, and in this way the complete pattern is transferred to the morphology of the film. In this thesis EBL is a key technique, since all nanopillars were patterned with a commercial Zeiss Supra 35 SEM in combination with a Raith Elphy-Plus lithography system. Therefore this section is devoted to a detailed description of the subsequential steps in a typical lithography process (figure 3.1), while a brief specification of the Zeiss Supra 35 SEM is given in chapter 3.2.2.

The process starts with spin-coating the electron sensitive resist onto the substrate. Afterwards the sample is baked out either on a hot plate or in a convection oven in order to evaporate the solvents of the electron beam resist. Then the sample is ready for the writing process, for which it is transferred to the EBL tool. Here the electron beam is controlled from an 


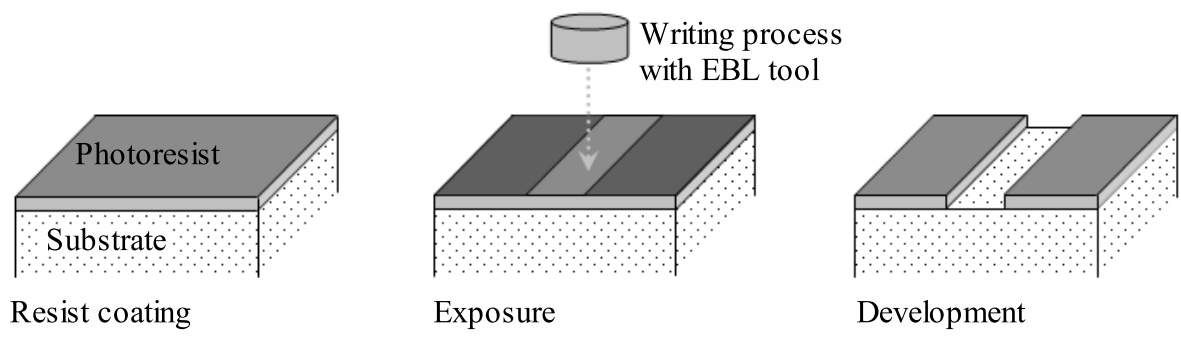

Figure 3.1: Relevant e-beam lithography process steps for a positive resist.

external computer to write the previously defined pattern in the electron beam resist. During this process step the electron irradiation locally breaks polymer backbone bonds in a positive resist, thus leaving fragments of lower molecular weight. On the other hand, for a negative resist the electron irradiation cross-links the polymer chains together. At this point it should be noted that also a positive resist might crosslink and form a negative resist upon exposure to extremely high doses of irradiation [46]. In the following process step the sample is dipped for a certain amount of time in a solvent developer, which selectively washes away the lower molecular weight fragments of the polymer. In this way a positive tone pattern is formed for a positive resist, while the contrary holds true for a negative resist, and the actual lithography process is finished at this point.
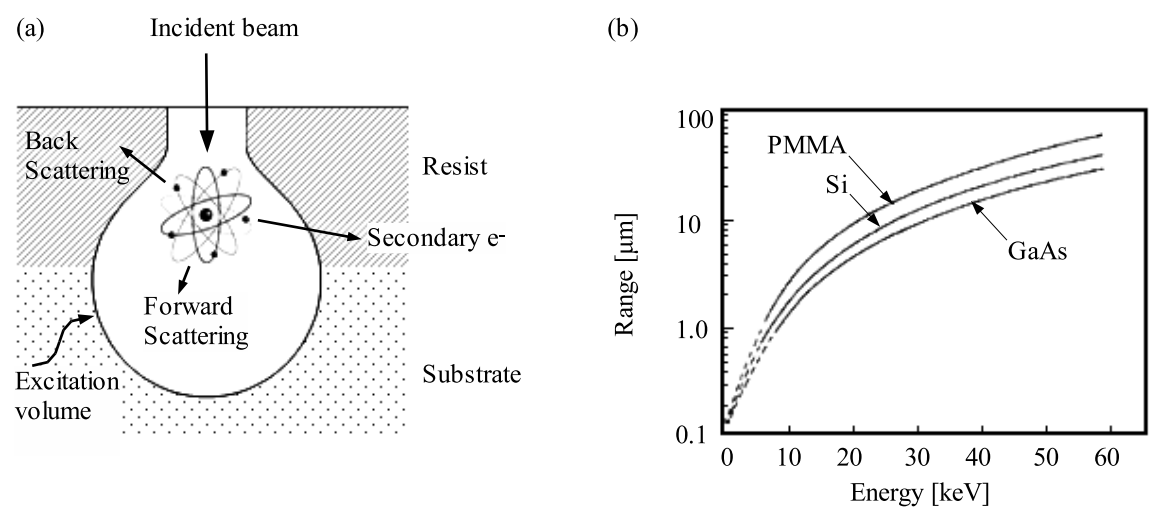

Figure 3.2: (a) Formation of an excitation volume in electron beam lithography. (b) Electron range as a function of beam energy for PMMA, Si and GaAs (taken from [51]).

This pattern can be transferred to the sample either by an etching step or by the evaporation of a metallic layer in combination with a subsequent lift-off step. Both processes are not relevant to the sample preparation described in section 3.1.3, since in this thesis the resist served only as an insulating layer with nanopatterned holes, in which the nanopillars were directly deposited. Nonetheless, they will be described here briefly. In the 
etching process the resist is resistant to the etching agent, so that in those areas of the sample, which are still covered by the resist, no material will be removed (this is also where the name 'resist' originally comes from). When no etching process is performed, but the pattern is transferred via evaporation, the reciprocal pattern will be formed: in the lift-off process the dissolver only removes the resist including the metal on top, but the metallic evaporated film directly on the substrate is not affected and thus remains.

The size of the patterned structures is the crucial point in electron beam lithography. The resolution of this technique does not really depend on the electron beam diameter of the lithography tool, but it is essentially determined by the interactions of the energetic electrons with the atoms in the electron-sensitive resist. Upon penetrating the resist, some electrons experience small angle scattering events (forward scattering), which effectively broaden the electron beam diameter at the bottom of the resist in comparison to its size at the surface. This effect can be minimized by choosing a very thin resist in combination with sufficiently high acceleration voltages during exposure. Far more important for the dimension of the interaction volume is a second effect: when the electrons continue their way into the substrate, many of them undergo large angle scattering events. These backscattered electrons may reenter the resist at a significant distance from the initial beam, thus causing additional exposure. This so-called proximity effect does not only depend on the type of substrate, but also on the energy of the primary electrons as it is depicted in figure 3.2 (b). Furthermore, secondary electrons play an important role. These are generated when the primary electrons knock other electrons out of the inner atomic shells, which in return ionize the material in their surroundings, so that secondary processes are initialized. Secondary electrons are responsible for most of the actual resist exposure activities, but because of their short range they contribute comparatively little to the proximity effect. Their net impact can rather be considered to lead to an effective widening of the beam diameter by roughly $10 \mathrm{~nm}$ [46].

\subsubsection{The Ultra High Vacuum System}

Most parts of the sample preparation process for this work were carried out in an ultra high vacuum (UHV) system. This setup is motivated by several factors. Initially the most important and obvious one is that for the samples of interest here, interface effects play a crucial role. In fact, contamination by adsorbed atoms could either alter or totally destroy the phenomena under investigation. Furthermore, the preparation of prototypical samples with a high quality crystallographic structure allows a better comparison of the experimental results with theoretical calculations. The third general point regarding UHV is that it effectively reduces heat transfer between the evaporation source and the sample. Hence, a reliable temperature control during deposition, which is essential for high reproducibility, becomes possible. 

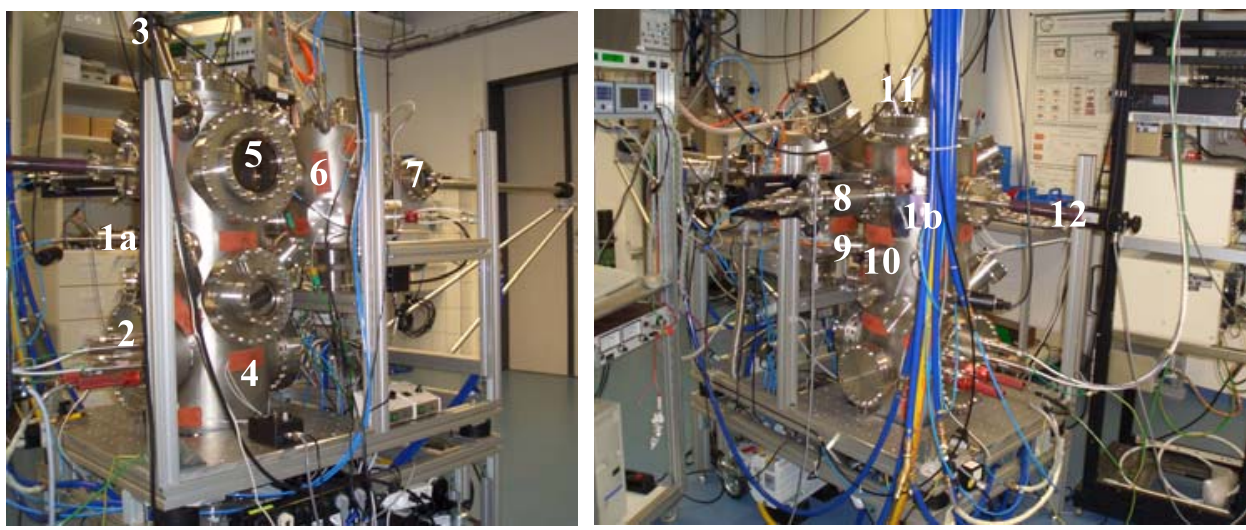

Figure 3.3: (a) Photographies of the UHV system (front and back view). The numbers are explained in the text.

For these reasons a UHV system as it is depicted in figure 3.3 (the following numbers in the text refer to this figure) was constructed at the beginning of this dissertation. It comprises three separate chambers: a load lock (7), a medial chamber for sputtering (6) and the main chamber (4) for e-beam evaporation and in-situ characterization. While the load lock is a standard constructional element, the two other chambers are custom-made by the company Trinos in Göttingen. All chambers possess independent Pfeiffer pumping systems each consisting of a a rotary pump in combination with a turbomolecular pump with a throughput of 60,210 and $500 \mathrm{l} / \mathrm{s}$, respectively. After $48 \mathrm{~h}$ of bake-out at $150^{\circ} \mathrm{C}$ the base pressure in the main chamber amounts to below $5 \cdot 10^{-10}$ mbar. This is controlled by a Pirani pressure gauge (11) in combination with a hot cathode ionization gauge (9) for lower pressure ranges in each deposition chamber (bought at AML). Additionally, a partial pressure analysis is facilitated by a Pfeiffer quadrupole mass spectrometer (3) installed in the main chamber. This device is also used for He leak detection. For a faster sample exchange, better contamination prevention and easier repair work all three chambers are separated by fully automatic electromagnetic valves (purchased at VAT). In the same way each turbomelcular pump can be separated from the system.

While the two major components of the main chamber, the electron beam evaporation tool (2) and the sample holder (8), are described separately in the following subsections, a few more important details about the chamber itself will be described right away. For a sufficient thickness and rate control of the evaporated film, two Inficon XTC/2 quartz crystal microbalances are mounted here (1a and $1 \mathrm{~b}$ ). The lower one is located at a fixed position, but the upper one is installed on a manipulator so that it can be moved to the potential sample holder position prior to deposition. Thus the tooling factor (i.e. the difference in material deposited on the lower quartz sensor versus the substrate) can be measured in situ, and the much more timeconsuming process of externally determining the actual film thickness for instance by means of x-ray diffraction analysis can be avoided. Another 
important feature of the evaporation chamber is the large non-distorted viewport (5) at its front, which will allow in situ MOKE investigations on the magnetic films in the future.

For maximum flexibility the entire UHV system is mounted on a compact chassis, which is divided into two parts, so that the upper part can be oscillatory decoupled by means of four vibration compensators.

\section{Electron Beam Evaporation}

All metallic thin film stacks presented in this work have been deposited by evaporation with two $3 \mathrm{~kW}$ multiple crucible linear e-guns purchased at Thermionics. The identical tools are mounted back to back with two magnetical shieldings in between as it is shown in figure 3.4 (a), so that altogether eight different materials can be evaporated. Indexing of the different crucibles over the fixed position of the electron beam is accomplished by turning the positioning handles on the outside of the system. If necessary, co-evaporation of two materials can also be performed simultaneously.

(a)

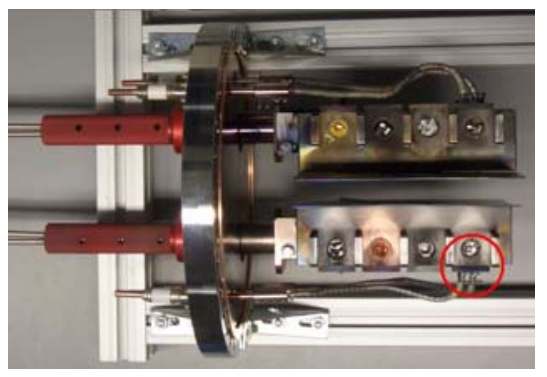

(b)

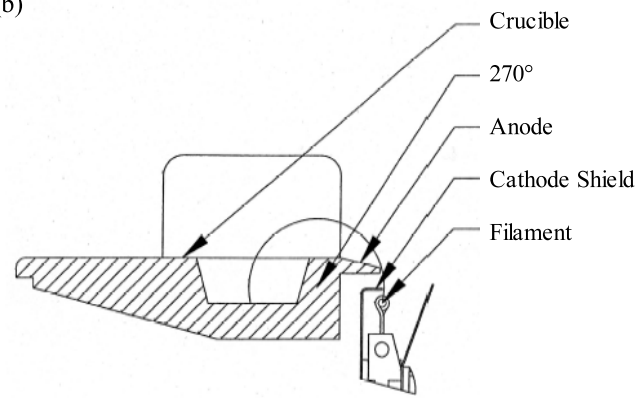

Figure 3.4: (a) Photography of the e-gun system (dismounted from the $U H V$ chamber for maintenance). A schematic drawing of the crucible in the red sector is shown in (b).

Figure 3.4 (b) shows the basis of the e-beam approach. The material to be evaporated is placed in the crucible of a water-cooled hearth. A thermionic tungsten filament generates electrons, which are formed to a beam by the geometry of the cathode shield and then accelerated by the high potential of the cathode. To remove the electron source from the line of evaporation and to prevent impurities from the filament reaching the pure deposition material, a magnetic field from a built-in permanent magnet bends the electron beam towards the crucible. The exact beam position on the material can be laterally aligned by slightly changing the high voltage with a so-called beam adjust. At an accelerating voltage of $4 \mathrm{kV}$ and an emission current of $750 \mathrm{~mA}$ a total power of $3 \mathrm{~kW}$ can thus be transferred to a hot spot with a diameter of approximately $1 \mathrm{~mm}$. As a result the material melts locally and evaporation starts. For highly thermally conductive materials such as $\mathrm{Cu}$ or $\mathrm{Au}$ it is advantageous to place a thermal barrier between the hearth and the melt in order to lower the beam power 
and to evade heavy deposits or cross contamination in the crucible itself. For this purpose crucible liners made of graphite or glassy coated graphite were utilized.

\section{The Sample Holder}

A custom-made sample holder which provides an accurate temperature control as well as flexible substrate and mechanical mask positioning has been designed for the main chamber of the UHV system. The result is depicted in figure 3.5.

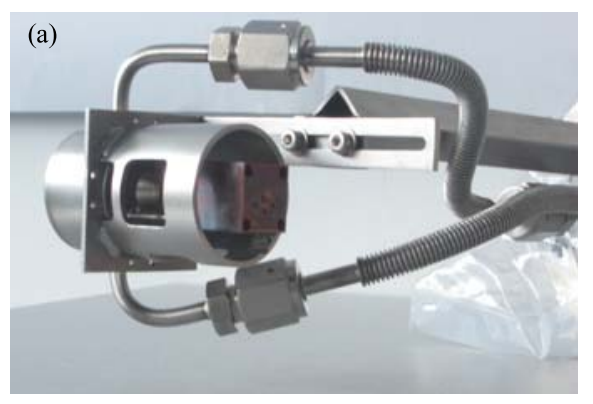

(b)

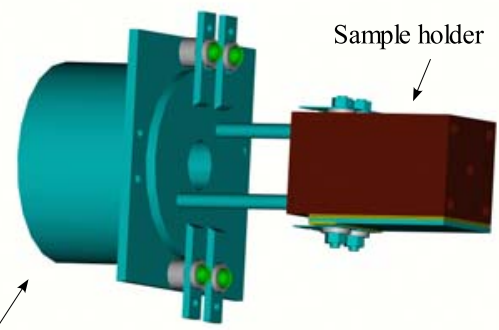

Sample receiving station

Figure 3.5: (a) Photography of the sample holder and the sample receiving station. A corresponding schematic drawing is presented in (b). Adapted from [47].

The sample holder itself consists of a hollow $\mathrm{Cu}$ cuboid. On its four larger lateral surfaces the substrates and mechanical masks can be fixed by means of clamps and screws. One of the two smaller heading sides can be connected to the transfer rod by means of a screw thread, while its other side features four holes for the parallel pins of the appendant sample receiving station. These stainless steel pins also link the sample holder thermally to the cooling reservoir which is integrated into the sample receiving station. By having liquid nitrogen flow through this reservoir, the sample can be cooled down to a temperature of approximately $80 \mathrm{~K}$. For maximum cooling capacity an additional heat shield, which is thermally coupled to the cooling reservoir as well, surrounds most of the surface of the sample holder. For higher temperatures a conventional filament is used as a heater. It is mounted on the sample receiving station, but juts out into the cavity of the sample holder, so that temperatures up to $650 \mathrm{~K}$ can be obtained here. Temperature control is performed by a standard type-K thermocouple, for which four electrical spring contacts are attached to the sample holder as soon as the latter one is connected to the receiving station.

The entire sample receiving station can be moved horizontally in order to position the sample exactly above the deposition crucible. This is especially important for the evaporation through a mechanical mask or the deposition into a template, because it guarantees a perpendicular angle of incidence of the impinging atoms. In this way shadowing effects are avoided and 
structures with steep side walls can be produced. Besides, the manipulation of the receiving station allows a transfer of the sample to a position very close to the optical window, so that in situ analysis becomes possible.

To avoid constant material deposition on the substrate (for instance during outgassing), two shutters are installed below the sample receiving station. The lower one (10) is opened by a rotary feedthrough and is used in the normal operation mode for covering the sample. The upper shutter has recently been mounted on a motorized linear feedthrough (12) whereby it can be gradually moved through the path of the evaporating material. Hence, a preparation of wedge shaped samples has become possible.

\subsubsection{Sample Geometry and Preparation}

In this section the final design of the single- and trilayer ferromagnetic nanopillars is described from the bottom up. The corresponding schematic drawings can be found in figure 3.6. In contrast to earlier preparation techniques $[32,48]$, the fabrication method developed within this thesis is a simplified one step process, which does not require any ion milling or wet etching cycles.
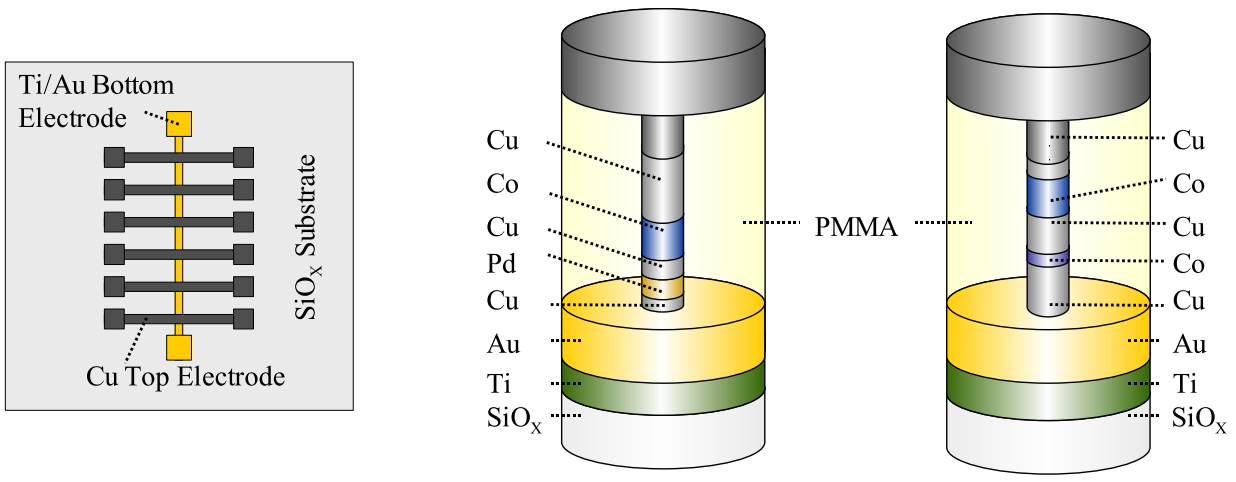

Figure 3.6: Top view on the sample and enlarged crossectional view. The pictures are not drawn to scale.

The samples are grown on thermally oxidized $\mathrm{Si}(100)$. Since the complete nanomagnetic elements are measured electrically, the most important characteristic of this substrate material is that it is not conductive, which is compounded by the cross-bar geometry used (see below). Besides, $\operatorname{Si}(100)$ can be cleaned and prepared easily, plus it is available as highly pure, very smooth, single-crystalline wafers. In a first preparatory step the $\mathrm{Si}(100)$ substrate is cut and cleaned in an ultrasonic bath with acetone and propanol.

The bottom electrode consists of a thin $\mathrm{Ti}$ layer with $\mathrm{Au}$ on top, which are deposited by means of thermal evaporation (cf. section 3.1.2). The Ti serves as an adherent layer for the $\mathrm{Au}$, which is a good choice for the electrode due to its excellent conductivity as well as its low tendency to oxidize. The vertical bottom contact structure is created by placing a simple shadow 
mask during the evaporation process directly on the substrate. The mask itself consists of a flat $0.5 \mathrm{~mm}$ thick Mo plate, with a $0.5 \mathrm{~mm}$ wide strip etched via spark erosion (left panel of figure 3.7 ). The larger squares at the ends of the bottom contact provide an area for electrical contacting, while the cross at the lower right corner serves as a marker for the subsequent patterning process (see below).
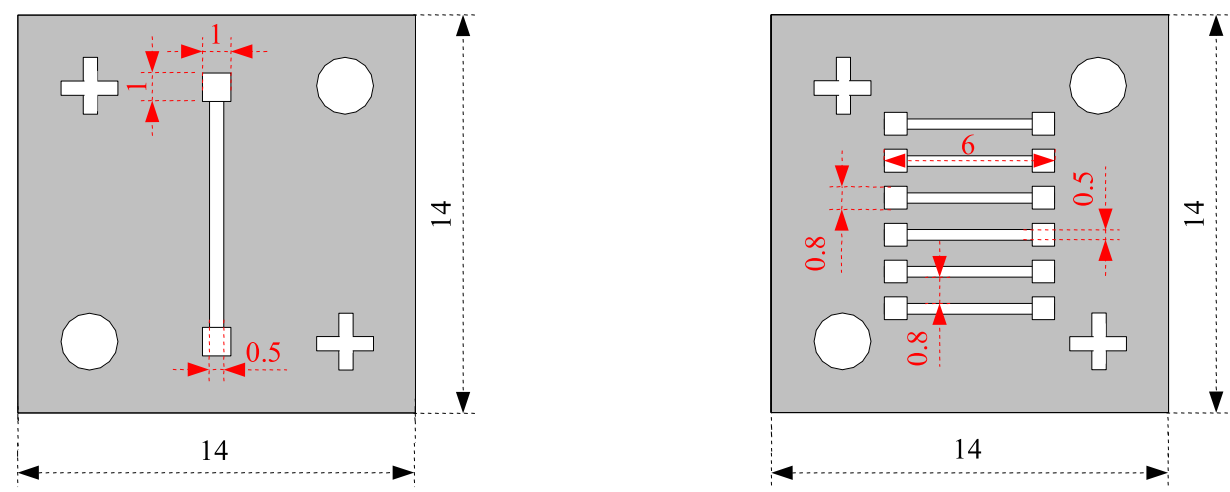

Figure 3.7: Shadow masks used for patterning the bottom and top electrodes.

After the deposition of the bottom electrode, the resist is spun onto the complete sample for 60 s at 2000rpm with a conventional spin coater technique. The resist is a solution of $2 \%$ polymethyl methacrylate (PMMA) in ethyl lactate with a molecular weight of 950K (Allresist AR-P 679.02), which is a standard positive electron beam resist with one of the highest resolutions available. Afterwards the sample is baked out at $170^{\circ} \mathrm{C}$ in a convection oven. By then the resist is a cohesive, relatively stiff, electrically insulating layer, which is ready for the e-beam exposure process that defines the actual pillar structures.

The e-beam patterning is performed with a Zeiss Supra 35 scanning electron microscope in combination with a Raith Elphy-Plus lithography system. For further technical details the reader is referred to section 3.2.2. During the lithography process six single dots, each of them positioned in the middle of the bottom electrode at a potential crossing point with the later deposited top contacts, are exposed. For this purpose the acceleration voltage of the electron beam is put to $20 \mathrm{kV}$ at a working distance of $10 \mathrm{~mm}$ and the dot dose amounts to $0.075 \mathrm{pAs}^{1}$. Apart from the dots several $100 \mu \mathrm{m}$ wide markers are exposed at a specific distance to the position of the dots, so that the correct location of the dots on the bottom electrode can be checked with an optical microscope later on, although the dots themselves are too small to be seen.

The e-beam patterning is followed by the development of the exposed structures. Methyl isobutyl ketone (MIBK) is an appropriate developer,

${ }^{1}$ The appropriate dot dose depends strongly on the thickness of the bottom layer and has to be carefully determined; cf. chapter 4 . 
but MIBK alone is too strong and might remove some of the unexposed photoresist. Thus, the developer is diluted by mixing one part MIBK to three parts isopropanol (IPA), which is a much weaker developer. The sample is dipped in this mixture for $98 \mathrm{~s}$, rinsed for another $45 \mathrm{~s}$ in pure IPA and thereafter blown dry in nitrogen. The result are six approximately $80 \mathrm{~nm}$ wide holes in the insulating PMMA plus the position markers right next to the bottom electrode.

Directly afterwards the sample is mounted on a $\mathrm{Cu}$ disk with the Mo mask for the definition of the horizontal cross contacts as depicted in figure 3.7 on top (To make sure that the top contacts cover the holes in the PMMA, the mask has to be exactly positioned by means of the cross marker at its lower right corner, which is matched with the cross marker deposited with the bottom contact.). Prior to the deposition of the actual layer stack in $\mathrm{UHV}, 10 \mathrm{~nm}$ of $\mathrm{Cu}$ are deposited at a high evaporation rate in the $\mathrm{HV}$ chamber. It was found that the adhesion of the layer stack on the PMMA could thus be improved. Only thereafter the entire construction is built in the load lock of the UHV chamber, which is pumped down to a pressure below $1 \cdot 10^{-6}$ mbar. Then the sample is transferred to the main chamber with a base pressure of $5 \cdot 10^{-10}$ mbar. There the actual layer stack of $3 \mathrm{nmCu} / 5 \mathrm{nmPd} / 5 \mathrm{nmCu} / \mathrm{xnmCo} / 30 \mathrm{nmCu}(\mathrm{x}=6-12)$ for the ferromagnetic single layer geometry or $13 \mathrm{nmCu} / 3 \mathrm{nmCo} / 10 \mathrm{nmCu} / 12 \mathrm{nmCo} / 30 \mathrm{nmCu}$ for the ferromagnetic trilayers is deposited by means of e-beam evaporation (see section 3.1.2). Typical evaporation rates, which can be monitored by a microbalance, are $1.3-1.5 \AA / \mathrm{s}$ for the $\mathrm{Cu}$ and approximately $0.8 \AA / \mathrm{s}$ for the Co and $\mathrm{Pd}$, respectively.

The top 30nm of $\mathrm{Cu}$ prevent the sample from oxidation upon transfer to the HV chamber, which is more appropriate for faster evaporation of larger amounts of material. Here the $\mathrm{Cu}$ is placed in a resistive tungsten boat which is traversed by a current. At sufficient currents ohmic heating of the boat leads to a melting of the source material. In this way the final $300 \mathrm{~nm}$ of the $\mathrm{Cu}$ layer are evaporated through the shadow mask on the top electrodes at a higher deposition rate of roughly $2.5 \AA / \mathrm{s}$. After removing the sample from the $\mathrm{HV}$ chamber and remounting the shadow mask the sample is ready for electrical contacting by a wire bonder.

\subsection{Characterization Techniques}

\subsubsection{Atomic Force Microscopy}

Atomic force microscopy (AFM) is one of the most prominent scanning probe microscopy techniques to obtain topographical information in real space. In principal this method provides atomic resolution, but imaging can also be performed on much larger areas, so that valuable information on the size and the distribution of single crystallites can be gained.

The basic principle of an AFM with its main components is depicted in figure 3.8. A sharp tip mounted on a soft cantilever is raster-scanned across the sample by means of piezoelectric translators, while measuring the 


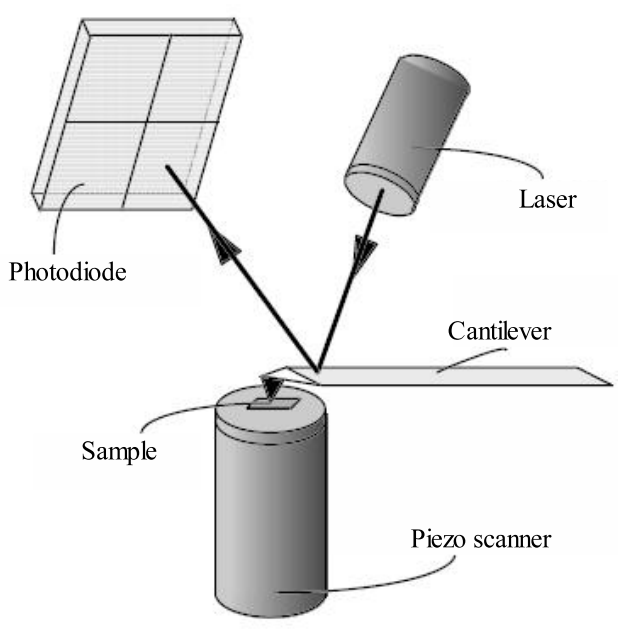

Figure 3.8: Schematic presentation of an atomic force microscope.

different forces between the tip and the surface for every single raster point. Imaging is then accomplished by measuring the deflection of the cantilever via a reflected laser beam on a photosensitive detector. In this way position changes of the cantilever of approximately $0.01 \mathrm{~nm}$ can be detected.

Signal generation is not really based on the interaction of single atoms, because the sample and the tip are large in comparison to the distance in between them. In order to estimate their interaction all the forces between the atoms of both bodies are added up. For a sphere with radius $R$ at a distance $d$ from a half-space this summation leads to an interaction potential of

$$
U=-\frac{A R}{6} \cdot \frac{1}{d}
$$

$A$ is the Hamaker constant, which is material specific and essentially contains the densities of the sample and tip material as well as the interaction constant $C$ of the van der Waals potential [49].

Since the distance dependence of $1 / d$ is relatively weak, a more sensitive measurement option has proven to be particularly favourable: in this socalled tapping mode the cantilever is driven near its resonance frequency by means of a piezo oscillator, so that the second derivative of the van der Waals potential is exploited. Far away from the sample the vibration frequency and amplitude are only determined by the nominal force constant of the bending cantilever, which corresponds to a harmonic potential. Upon approaching the surface this potential is superimposed by the van der Waals interaction potential, thus changing the vibration frequency and the amplitude of the cantilever. As a consequence the amplitude of oscillation is a precise measure for the distance of the tip to the sample surface.

For the completion of the measuring principle the force measurement has to be supplemented by a controlling unit. In the constant height mode the vertical position of the sample is kept at a constant value while the varying 
deflection of the cantilever is recorded. In this way comparatively high scan rates can be achieved at the expense of smaller scan sizes, since tip crashes are possible. In the alternative constant force mode the cantilever deflection is kept at a fixed setpoint value by readjusting the sample in vertical direction according to the topographic features on the surface in a feedback mechanism. In this way relatively large and rough surfaces can be analyzed without destroying the tip or the sample itself. On the other hand, only comparatively low scan rates are possible due to the time required for the system to respond to the height changes.

Thus the atomic force microscope can map areas of constant nominal force. If the surface is chemically homogeneous and if only van der Waals forces act on the tip, the image displays the topography of the surface.

\subsubsection{Scanning Electron Microscopy (Including the Lithography System)}

A schematic drawing of the Scanning Electron Microscope (SEM) tool, which essentially consists of an electron gun, the electron-optical system, a mechanical wafer stage and a controller system (plus an additional control unit for the lithography system), is shown in figure 3.9.

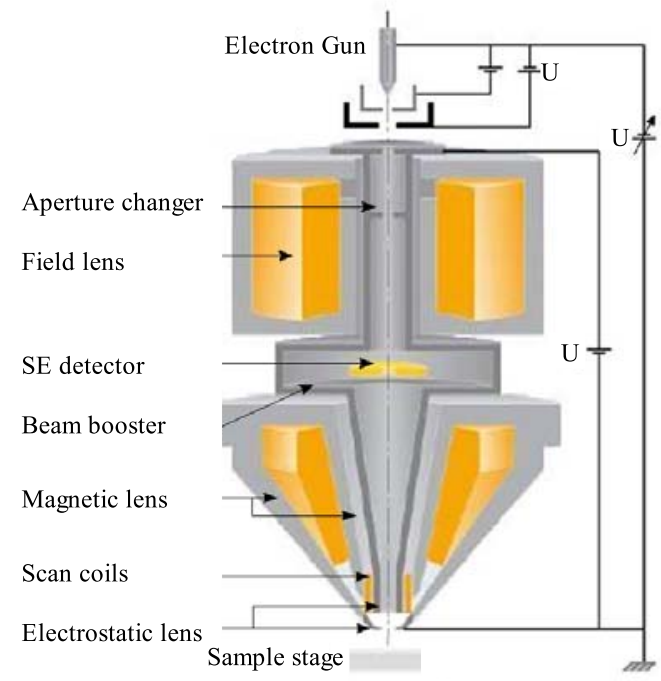

Figure 3.9: Schematic drawing of a scanning electron microscope. Adapted from [50].

In the Zeiss Supra 35 SEM the electrons are generated by a thermal field emission source, a so-called Schottky emitter. The source combines the field emission effect of a sharp W tip with thermal heating to $1800 \mathrm{~K}$, so that it is less sensitive to residual gases and extremely stable in operation. The $\mathrm{W}$ is additionally coated with $\mathrm{ZrO}$ in order to minimize the work function barrier. A heated reservoir of $\mathrm{ZrO}$ at the pole of the tip then continuously replenishes 
material evaporated from the tip. This kind of electron source guarantees a high brightness, but it also requires ultrahigh vacuum conditions ( $p<$ $\left.9 \cdot 10^{-9} \mathrm{mbar}\right)$.

In the electron column the electrons are formed to an accurately defined beam by means of several subsequent focusing and defocusing lenses and apertures. Both electrostatic and magnetic lenses entail spherical aberrations, where the outer zones of the lens focus more strongly than the inner zones, as well as chromatic aberrations, where electrons of slightly different energies get focused at different image planes. In order to reduce these aberrations, the convergence angle of the system is minimized as far as the reduced beam current permits. An important additional part of the electron column is the beam blank which consists of plates that are set up as electrostatic deflectors. Upon applying a voltage across these plates the beam is swept off axis and intercepted by a downstream aperture, so that the beam can be switched on and off with extremely fast response times. A similar technique is used for the beam deflection system installed in the column to raster-scan the beam across the surface of the sample.

Since this deflection system can only scan a field of approximately $(1000 \mu \mathrm{m})^{2}$, the sample can additionally be moved by a mechanical wafer stage.

The imaging process requires a proper detection method for the electrons. The most common imaging mode is to monitor low energy $(E<50 \mathrm{eV})$ secondary electrons, which are generated by inelastic scattering of the primary electrons at the atomic nuclei or the shell electrons of the sample material. These secondary electrons can be detected by the annular in-lens detector that is installed directly in the optical path above the objective lens of the SEM. Here the electrons impinge on a scintillator in combination with a photomultiplier, so that the resulting signal is rendered into an intensity distribution that can be viewed and saved as a digital image. While the in-lens detector provides the best high resolution data, an additional lateral secondary electron detector mounted at an angle of approximately $60^{\circ}$ to the electron path at the wall of the chamber provides optimum topographical resolution. For a better chemical contrast higher energy backscattered electrons $(E>50 \mathrm{eV})$ can also be detected. These can be observed especially when the sample species strongly vary in their atomic number by means of a four quadrant backscattering detector. To achieve an optimized image quality the signals from all detectors can be mixed electronically in any ratio.

Since the SEM is not only used for imaging but also for writing processes, the pattern generator unit Elphy-Plus by Raith plays a key role for the ebeam lithography. The primary control of the system consists of a PC, which transmits only the coordinates of the corners of the corresponding structure to a separate computer and integrated DAC as the actual pattern generator. This patterning hardware then generates all of the internal points for exposure, so that the data throughput of $2.6 \mathrm{MHz}$ is comparatively high. However, the speed of the entire writing process is eventually limited by the SEM deflection system to a much lower frequency, because the inductance 
of the coils and low pass filters of the Zeiss Supra 35 have to be taken into account.

\subsubsection{Transmission Electron Microscopy}

While the SEM described above is essentially used to investigate surface morphology, the transmission electron microscope (TEM) is a prominent tool for directly exploring the internal structure of a specimen by analyzing the electrons passing through the material. Since electrons are highly absorbed by almost any kind of material the samples have to be extremely thin: $500 \mathrm{~nm}$ or less for low resolution experiments and approximately $10 \mathrm{~nm}$ for high resolution images, respectively. For this reason comparatively complex sample preparation techniques are inevitable; one of them is outlined in section 3.2.4.

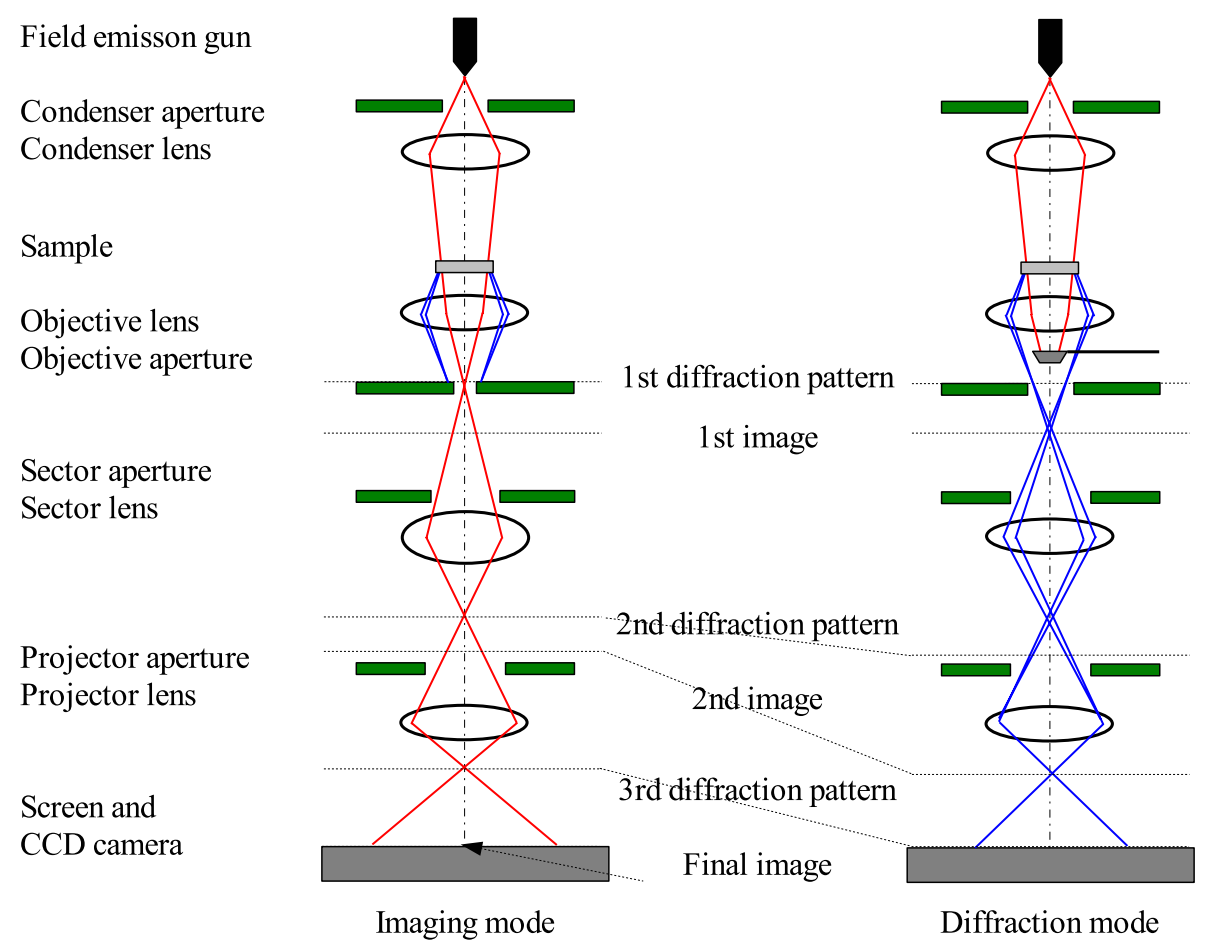

Figure 3.10: Arrangements of the various components of a TEM with a ray diagram for the imaging mode (left) for information in real space and the diffraction mode (right) for information in reciprocal space.

A schematic drawing of a TEM similar to the one used for this thesis (Philips CM200-FEG-UT microscope) is shown in figure 3.10. The field emission gun generates a diverging beam of electrons through the anode hole. This beam is then focused down to a fairly small spot size by the condenser system. After passing the sample, the electrons are collimated by an objective aperture and an objective lens. Thus particular groups of electrons which contribute to the final image are selected at this point. The 
following intermediate lens is used to magnify the image or the diffraction pattern. For this reason two different modes can be chosen here: the microscopic imaging mode (figure 3.10 left) or the analyzing diffraction mode (figure 3.10 right). Ultimately, the projector lens images the electrons on a computer-controlled CCD camera, which records the final image.

This TEM image can be produced by different interactions of the electrons with the specimen. The following cases are of great importance:

- transmission without interacting with any atom

- elastic scattering

- inelastic scattering (as a result secondary electrons, phonons, UV light or cathodoluminescence may be produced; for inner shell electrons this leads to the emission of x-rays or Auger electrons).

If the electrons of both interaction types contribute to the image, all regions of the specimen will look the same and no contrast will be seen for areas of different composition or thickness. For this reason the electrons are separated by the aperture at the back focal plane of the objective lens. Which contrast mode is chosen depends on the problem under investigation.

The bright field mode with an aperture placed in the back focal plane of the objective lens lets only the direct beam pass. In this way essentially mass-thickness and diffraction contrast contribute to the formation of the image: thick areas, regions in which heavy atoms are enriched, and crystalline areas appear with dark contrast.

For dark field images the direct beam is intercepted by the objective aperture. Thus the diffracted beam produces the image. Due to its strong interaction with the sample, valuable information on the crystal grain size and orientation, planar defects or stacking faults in the material can be obtained.

By choosing a comparatively wide objective aperture so-called phase contrast, which is produced by the interference of the diffracted beams with the direct beam at the image point, can be observed. In combination with a sufficient point resolution TEM high-resolution images are formed, so that in principle atomic resolution can be achieved for suitable samples.

Another important mode applied in this thesis is the scanning transmission electron (STEM) option, when a small convergent electron beam is scanned over a defined area of the sample. In this way a highly localized signal can be gained from the specimen; here the emitted energy dispersive x-rays (EDX) are recorded for each scanning point. Since the corresponding detector can be tuned to certain wavelengths characteristic for each element, the chemical structure can be locally analyzed.

\subsubsection{Focused Ion Beam}

High resolution imaging and quantitative analysis by means of TEM requires complex sample preparation methods, because micron-sized sections 
of uniform thickness have to be produced. A focused ion beam (FIB) system allows for precise material removal by ion beam sputtering and targeted material deposition (gas-assisted). Since it also facilitates an accurate selection of the region of interest by simultaneous inspection of the species, the FIB is a very convenient tool for the preparation of TEM lamellas.

For this thesis the FIB work was performed with the dual-beam FIB/SEM system Nova 600 NanoLab manufactured by FEI as it is depicted in figure 3.11. Its mode of operation is actually quite similar to the one of a SEM (cf. section 3.2.2); the major difference is that the primary beam consists of $\mathrm{Ga}^{+}$ions instead of electrons. The interaction of these ions with the sample surface leads to the generation of secondary ions which are used for imaging with a spatial resolution of about $7 \mathrm{~nm}$ [52]. Due to channeling of the ions the contrast depends on the crystallographic orientation of the sample. This allows for a relatively easy identification of individual grains in polycrystalline samples. For a somewhat higher resolution or for an improved contrast electron-optical imaging with the integrated SEM can be applied. In addition, the FIB/SEM tool is equipped with an in-situ gas injection system (here used for the deposition of $\mathrm{Pt}$ ) as well as an in-situ micromanipulator.

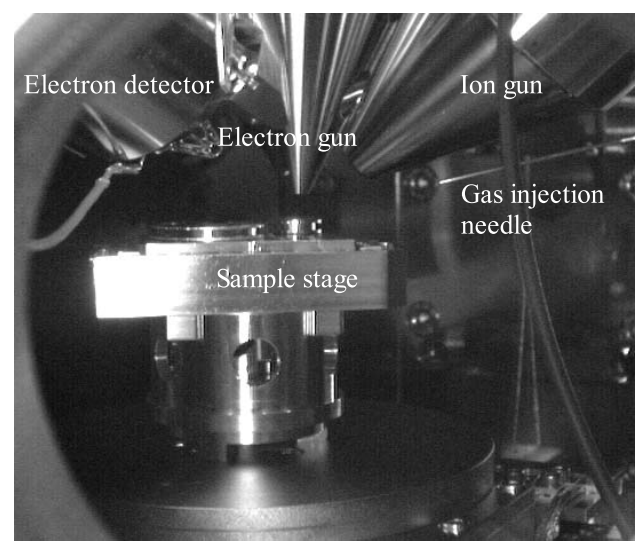

Figure 3.11: Inside the chamber of the dual-beam FIB/SEM tool.

In the following paragraph the preparation of a cross-section TEM specimen from a bulk sample by the so-called lift-out technique will be described chronologically.

In a first step the area of interest on the top of the specimen is located in the electron imaging mode. Then the gas needle is drawn near the sample surface, and a Pt metalorganic precursor is injected. This is absorbed on the target surface, where it is decomposed by the rastering electron beam and in a second stage by the scanning ion beam. In this way a line of Pt $(2 \mu \mathrm{m} \mathrm{x}$ $15 \mu \mathrm{m}, 250 \mathrm{~nm}$ plus $3 \mu \mathrm{m}$ in height) is deposited at the desired specimen region in order to prevent damage and spurious sputtering during FIB milling (see figure 3.12), while the byproducts of the precursor are removed through the vacuum system. Afterwards large stair-step FIB trenches are cut on both 


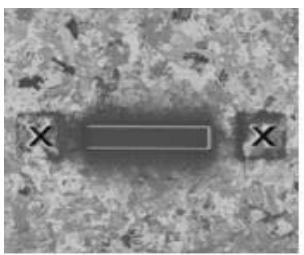

(a)

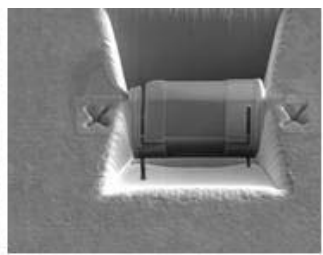

(b)

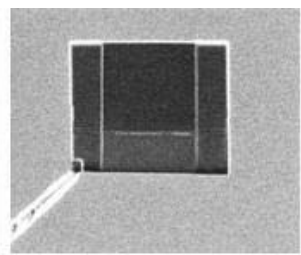

(c)

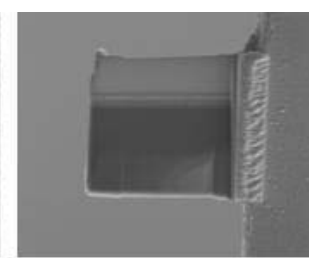

(d)

Figure 3.12: The FIB lift-out technique: (a) deposited Pt layer and marks for FIB milling (ion image) (b) stair-step trenches (electron image) (c) sample transport with the micromanipulator (ion image) (d) lamella attached to the TEM mesh grid (electron image).

sides of the area of interest parallel to the $\mathrm{Pt}$ deposit in order to create a $1.5 \mu \mathrm{m}$ thick lamella. During milling the FIB current is subsequently decreased from $\mathrm{I}_{\mathrm{Ga}}=5 \mathrm{nA}$ to $0.1 \mathrm{nA}$ (at $30 \mathrm{kV}$ ) upon approaching the $\mathrm{Pt}$ deposit. Prior to the final thinning, the sample is tilted to $51^{\circ}$ (between the surface normal an the incident ion beam) and the left side, the bottom and a part of the right side of the sample are cut free with $\mathrm{I}_{\mathrm{Ga}}=300 \mathrm{pA}$. Then the micromanipulator is approximated to one edge of the almost freestanding lamella until contact is made, so that the tip of the micromanipulator can be attached to the sample by ion beam induced deposition of Pt. Afterwards the remaining right side of the specimen is milled free, and the membrane is lifted out of the bulk sample and positioned onto a carbon coated $\mathrm{Cu}$ TEM mesh grid. Here it is again fixed by ion beam induced deposition of Pt on both sides of the sample. Thus the micromanipulator can be cut free and withdrawn from the lamella. Only then the final milling steps are performed (again from both sides) in order to thin the membrane to electron transparency, which corresponds to a thickness of maximal 100nm and much less for high-resolution TEM experiments. The final FIB cut is accomplished with $\mathrm{I}_{\mathrm{Ga}}=30 \mathrm{pA}$ and $5 \mathrm{kV}$ at $0.7^{\circ}$ with respect to the plane of the specimen surface. Hence, the amorphized outer layer of the specimen as well as implanted ions in it can at least partially be removed.

\subsubsection{Experimental Setup for Transport Measurements}

Since the samples produced for this thesis have a comparatively low resistance of approximately $1 \Omega$ and resistance changes of less than one percent have to be resolved, an extremely sensitive measurement technique is required for these experiments. This makes AC transport measurements, for which the differential resistance $d V / d I$ is recorded as a function of the DC bias voltage $V$, the method of choice. Initially these experiments were supposed to be performed with a commercial Physical Property Measurement System (PPMS) manufactured by Quantum Design. First tests revealed that due to an extremely poor signal-to-noise ratio the electronics of this instrument cannot detect resistance changes in the range of several $\mathrm{m} \Omega$ in spite of an implemented AC resistivity function. 


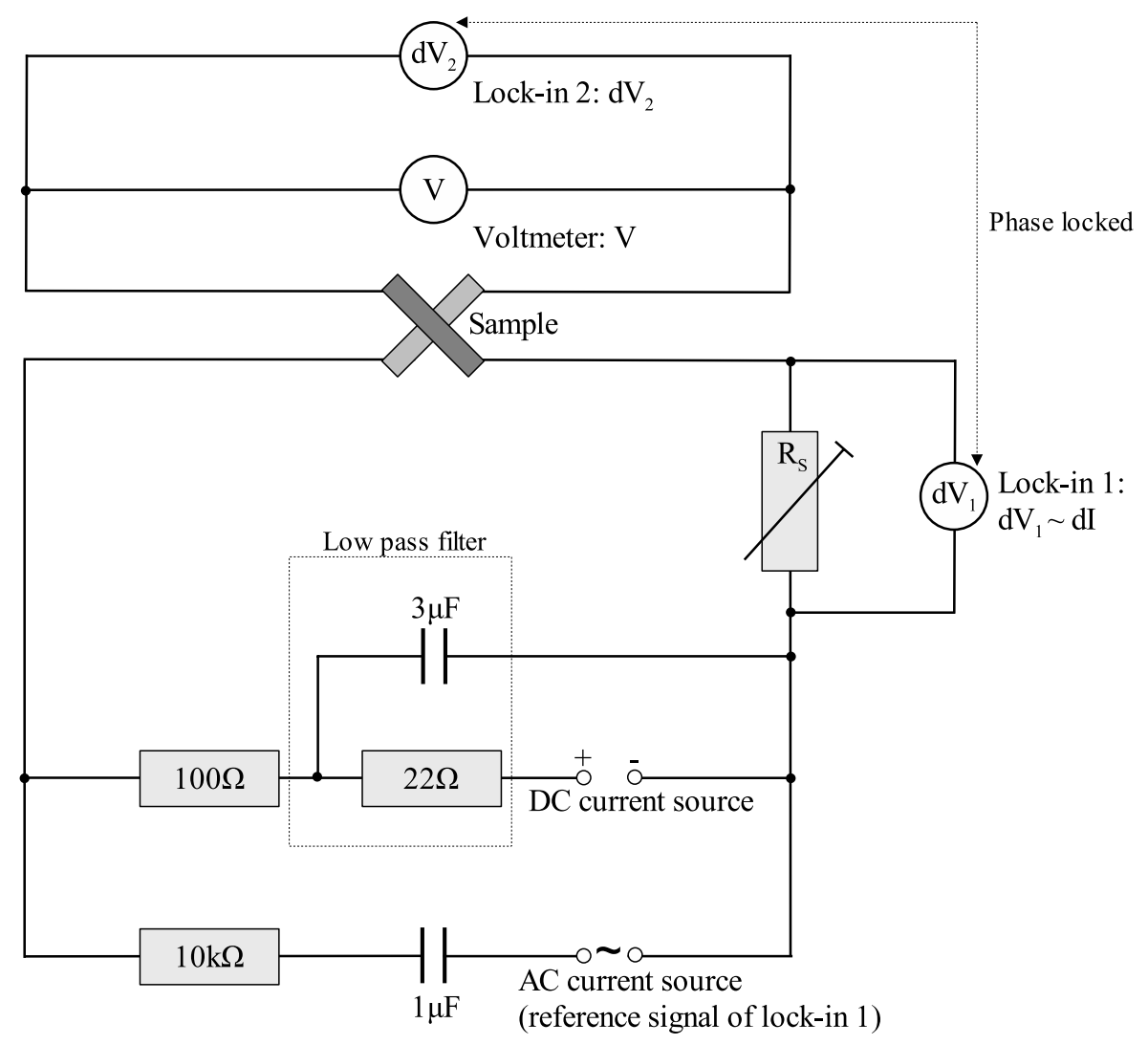

Figure 3.13: Electronic circuit designed for the simultaneous measurement of differential resistance $d V / d I$ and $D C$ voltage $V$.

Hence, a completely self-built external electronic circuit was designed in order to detect the $\mathrm{AC}$ resistance of the sample in a four-point measurement geometry (see figure 3.13). A small AC current of $100 \mu \mathrm{A}$ is added to an adjustable DC current of several $\mathrm{mA}$. For this purpose a sinusoidal voltage supplied by a first Stanford Research Systems lock-in amplifier SRS 830 is added to the circuit. In order to minimize its influence on the output of the DC current source (Keithley 2400 Sourcemeter), the latter one is protected by an appropriate low pass filter as indicated in figure 3.13. In turn, the sinusoidal output of the lock-in amplifier is connected in series with a capacitance of $1 \mu \mathrm{F}$ in order to shield it from excessive $\mathrm{DC}$ voltages. Since the DC sourcemeter has a relatively low internal resistance, a $100 \Omega$ resistor is wired in line with it to avoid a complete potential drop of the $\mathrm{AC}$ voltage here. Thus the AC voltage will predominantly drop across the sample. An additional resistor $R_{S}$ allows for the calibration of the currents flowing through the sample. Its value should be comparable to the sample resistance, because too high values of $R_{S}$ will cause a complete DC voltage drop at this point and not at the sample. On the other hand, too low values of $R_{S}$ will strongly decrease the voltage detected parallel to $R_{S}$ with the first lock-in amplifier, which might eventually lead to a deteriorated signal-to- 
noise ratio. The voltage measured with this lock-in amplifier is proportional to $d I$, so that $d I$ can be directly derived from

$$
d I=\frac{d V_{l o c k-i n 1}}{R_{S}}
$$

In order to deduce the total differential resistance $d V / d I, d V$ has to be measured separately. This is done by a second Stanford Research Systems lock-in amplifier SRS 830, which is phase locked to the first one, as shown at the top of figure 3.13. Simultaneously with this AC voltage the DC output voltage across the sample is detected by a Keithley 2000 Multimeter. From this signal the DC current can be computed, so that all values for a detection of the differential resistance $d V / d I$ as a function of DC current $I$ are complete. Standard parameters for the lock-in amplifiers are a modulation frequency of $7013 \mathrm{~Hz}$ due to an optimum signal-to-noise ratio for the hardware configuration described above and time constants of 300ms.

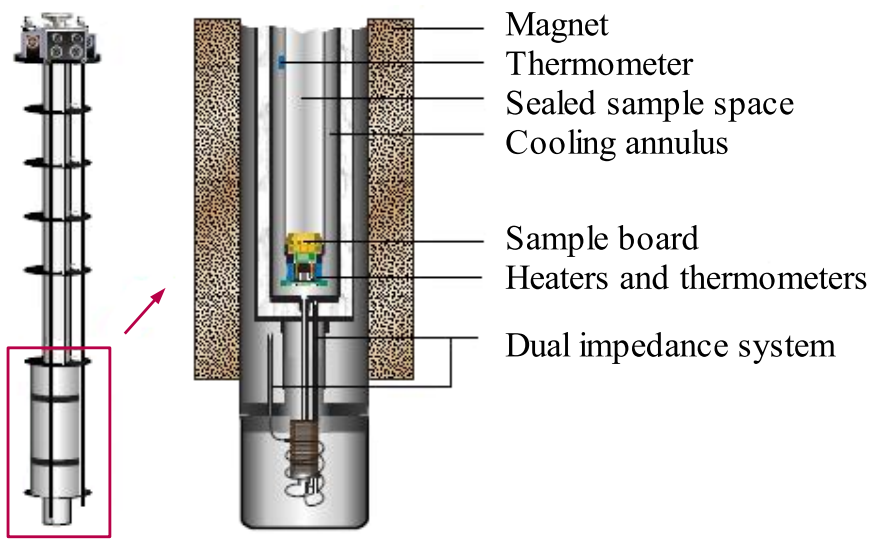

Figure 3.14: PPMS probe incorporating the magnet, the temperature control, the sample board and the sample connectors, respectively. Adapted from [53].

For measurements at low temperatures and high magnetic fields, the samples can still be mounted in the high vacuum chamber of the PPMS. For this reason they are fixed on a standard PPMS rotator sample board. Electrical connections are attached by means of ultrasonic wire bonding using $50 \mu \mathrm{m}$ thick Al wires. The cryostat integrated in the PPMS provides a precise temperature control in the range of $1.9 \mathrm{~K}$ to $400 \mathrm{~K}$, allowing for constant temperature measurements as well as for smooth temperature sweeps. At the same time, magnetic fields of up to $9 \mathrm{~T}$ can be applied by means of a conventional superconducting magnet. Additionally, a horizontal rotator option provides the possibility to rotate the sample from $-10^{\circ}$ to $370^{\circ}\left(0^{\circ}\right.$ corresponding to an out-of-plane direction of the applied field), thus yielding information about angular magnetic field dependence. 


\subsubsection{Magnetic Photoemission Electron Microscopy}

One of the state-of-the art techniques for imaging magnetic domains with a high lateral resolution is magnetic photoemission electron microscopy (magnetic PEEM). In this approach photoelectrons are excited with circularly polarized synchrotron radiation, and then the effect of $\mathrm{x}$-ray magnetic circular dichroism (XMCD) in the emitted photoelectrons is exploited for magnetic contrast.

Figure 3.15 depicts the general technical layout of a PEEM tool. The xray beam is focused on the sample, where after photon absorption electrons are emitted at the surface (the depth of view is limited due to the inelastic mean free path of the photoelectrons of approximately $5 \mathrm{~nm})$. Since these electrons leave the sample at large angles relative to the surface normal, a high accelerating voltage is applied between the sample and the first electrode. Due to chromatic aberrations the electrons with proper trajectories have to be singled out by a contrast aperture, which is positioned in the back-focal plane of the objective lens. The rest of the electron-optical system essentially consists of several projective lenses in order to magnify the image onto a multichannel plate/scintillator crystal combination or a phosphor screen. In this way the electron image is converted into a photon image and can thus be recorded by a CCD camera. An improved control of the electron beam as well as a partial compensation of electron-optical imperfections can be achieved by additional elements, such as the deflector and stigmator units.

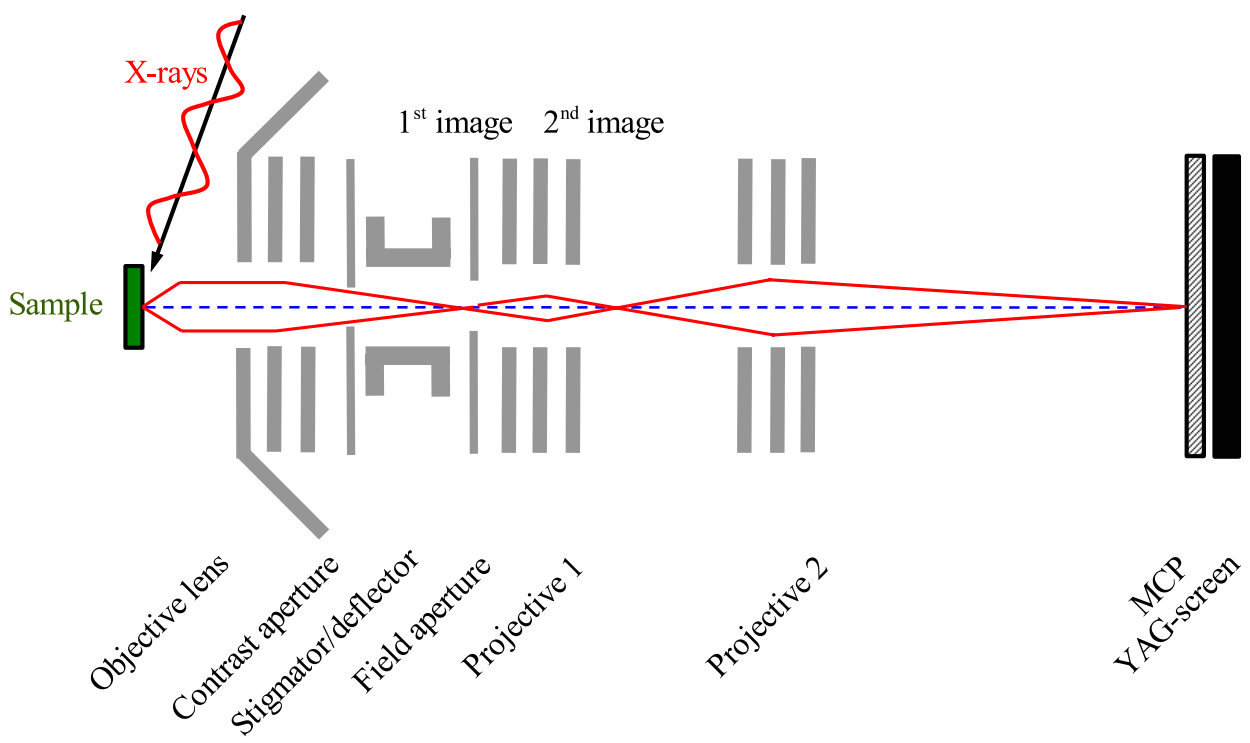

Figure 3.15: Technical layout of a conventional PEEM system. After [54].

The normal (i.e. non-magnetic) image contrast in PEEM essentially occurs due to the varying strength of the dipole matrix elements $M_{i f}=$ $\langle f|H| i\rangle$ across the imaged surface area. This variation arises either be- 
cause of local changes in the work function or the electronic structure (reflected in the initial and final electronic states $|i\rangle$ and $\langle f|$, respectively), or because of changes in the electric field vector $\vec{E}$ of the incident synchrotron light [54].

Magnetic contrast is now added to the PEEM signal by means of the XMCD technique, which relies on the asymmetry of resonant transitions between occupied core states and spin-split unoccupied states above the Fermi energy for left- and right circularly polarized photons. If the spin polarization of the transition, which is determined by the helicity of the exciting photons, is aligned with the spin polarization of the unoccupied states, the absorption cross section is relatively high, whereas it is much lower, if the spin polarization of the transition is antiparallel to the spin polarization of the unoccupied states $[55] .^{2}$ This difference in absorption cross section of the XMCD signal is then translated to a dichroism in the Auger electron yield. For a sufficient energy discrimination in the PEEM system this signal can already be used for a magnetic image; otherwise the helicity-dependent difference in the secondary electron yield has to be exploited.

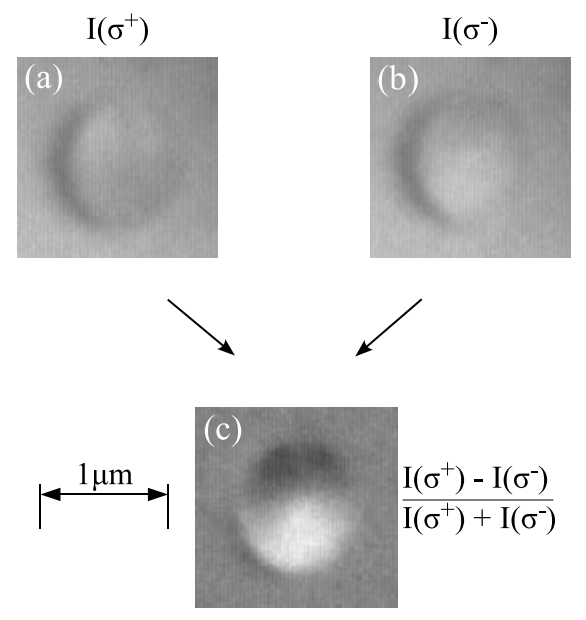

Figure 3.16: Example for magnetic contrast in XMCD PEEM for a $1 \mu \mathrm{m}$ wide, 10nm thick Co pillar covered with $2 \mathrm{~nm} C u$ : (a) image obtained with positive helicity (b) same image with negative helicity (c) asymmetry image calculated from (a) and (b) due to equation 3.3.

Figure 3.16 visualizes how magnetic contrast is achieved in magnetic PEEM experiments, which were performed at the SIM beamline of the Swiss Light Source in Villigen, Switzerland. Here an XMCD PEEM image of a $3 \mathrm{nmCu} / 5 \mathrm{nmPd} / 5 \mathrm{nmCu} / 10 \mathrm{nmCo} / 2 \mathrm{nmCu}$ pillar with a diameter of approximately $1 \mu \mathrm{m}$ is taken with positive photon helicity $\sigma^{+}$(a). The same field of view is recorded again for the opposite helicity $\sigma^{-}$(b). Then the so-called asymmetry $A$, which can be derived by calculating the intensity difference

\footnotetext{
${ }^{2} \mathrm{~A}$ concise treatment of the XMCD process can be found elsewhere [56, 57].
} 
of images (a) and (d) divided by their sum, yields a quantitative measure of the XMCD effect (c):

$$
A=\frac{I\left(\sigma^{+}\right)-I\left(\sigma^{-}\right)}{I\left(\sigma^{+}\right)+I\left(\sigma^{-}\right)}
$$

Dark and bright areas in image (c) correspond to regions of parallel and antiparallel local magnetization orientation with respect to the light helicity vector. Areas with a different magnetization direction can be classified on the basis of their gray level. Hence, a vortex state of the magnetization can be clearly identified in figure 3.16 (c). 


\section{CHAPTER 4}

\section{Structural and Magnetic Characterization of the Samples}

This chapter starts out with one of the key issues of the e-beam lithography process: the determination of the correct exposure dose. The effective dot dose does not only depend on the beam current and the accelerating voltage of the lithography tool, but it is also very sensitive to the specific properties of the material beneath the photoresist - especially to the thickness of the bottom electrode. For this reason a characterization of the bottom electrode material is performed in advance: here the thickness parameters which allow for smooth growth of the subsequent layers are investigated. In the second part of this chapter the outcome of the optimized lithography process is analyzed, i.e. the dimensions and shapes of the nanopillars are determined by means of SEM and TEM investigations. Besides, the magnetic structure within the nanopillars is studied in XMCD-PEEM experiments. The corresponding results are completed by micromagnetic simulations.

\subsection{Characterizing the Bottom Electrodes and the Corresponding Lithography Parameters}

Spin-dependent scattering processes in multilayers are known to be extremely sensitive to different interface roughnesses; for example, Kano et al. [58] as well as Suzuki and Taga [59] report that the GMR ratio of $\mathrm{Co} / \mathrm{Cu}$ superlattices significantly decreases upon increasing interfacial roughness. This suppression of GMR is probably due to a change in the magnetic state of the Co atoms in the intermixed regions, i.e. the magnetic moments of these atoms might be reduced and misaligned with the magnetization of the Co layer [60]. Hence, the growth conditions as well as the structure of the interface play a crucial role when investigating spin torque phenomena. 


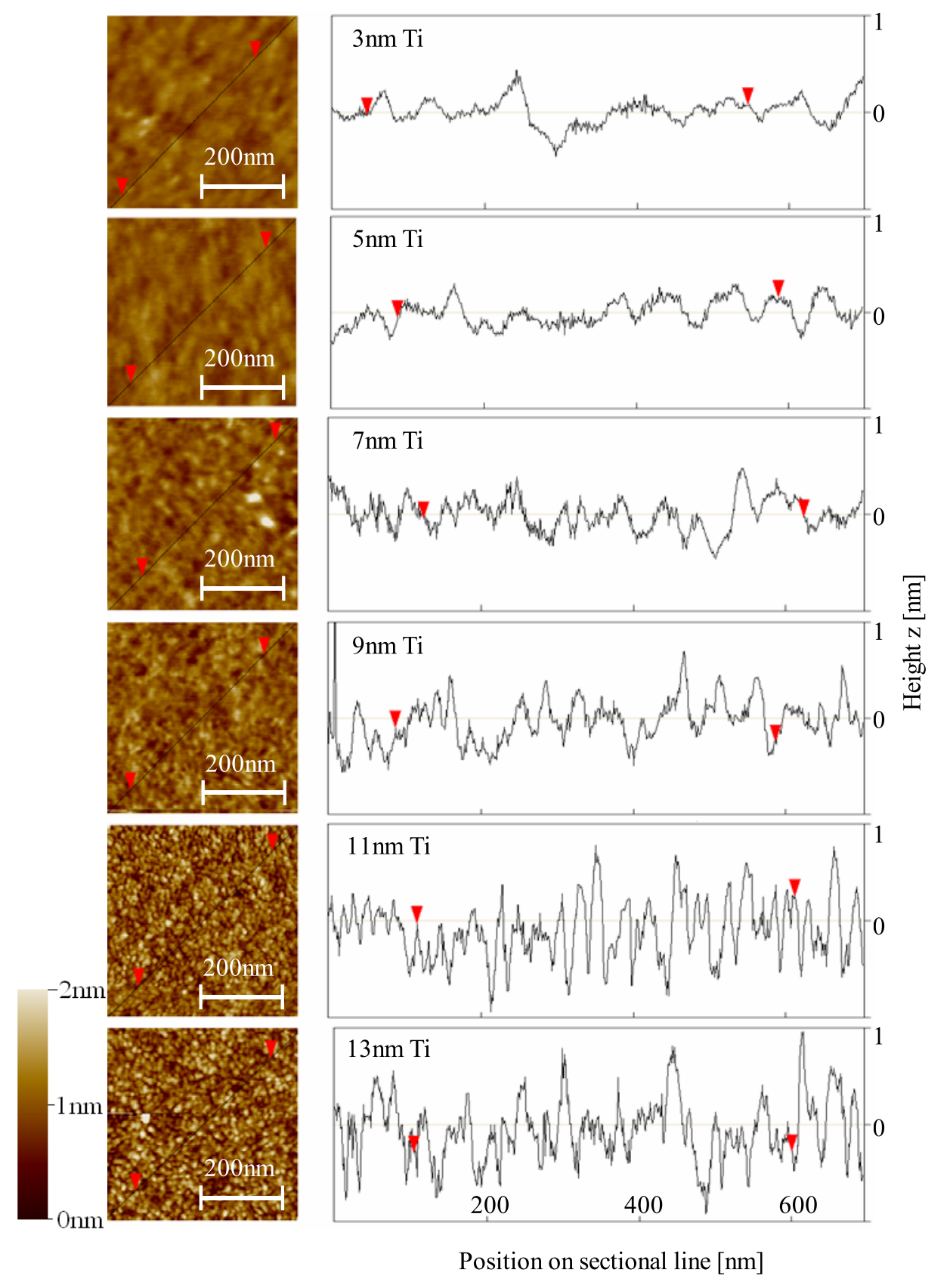

Figure 4.1: AFM measurements on Ti samples of different thicknesses. $R M S$ roughness and a sectional line were analyzed for each sample.

For these reasons the surface roughness of the $\mathrm{Ti} / \mathrm{Au}$ bottom electrodes has to be minimized in order to guarantee a smooth growth of the subsequent $\mathrm{Cu} / \mathrm{Co}$ layers. The corresponding results are presented and discussed within this section. Figure 4.1 shows AFM measurements on Ti bottom contacts of different thicknesses prepared by e-beam evaporation. The measurements were performed in the tapping mode with scan areas of $500 \mathrm{~nm} \cdot 500 \mathrm{~nm}$. The 
sectional line through each topography clearly indicates that the surface roughness of the Ti increases for larger thicknesses. This is confirmed by the RMS roughness data, which is displayed in figure 4.2 as a function of $\mathrm{Ti}$ thickness: here the two parameters are shown to be directly proportional to each other. Since the Ti only serves as an adhesion layer for the $\mathrm{Au}$, the smoothest Ti layer with a thickness of $3 \mathrm{~nm}$ is chosen for the following experiments.

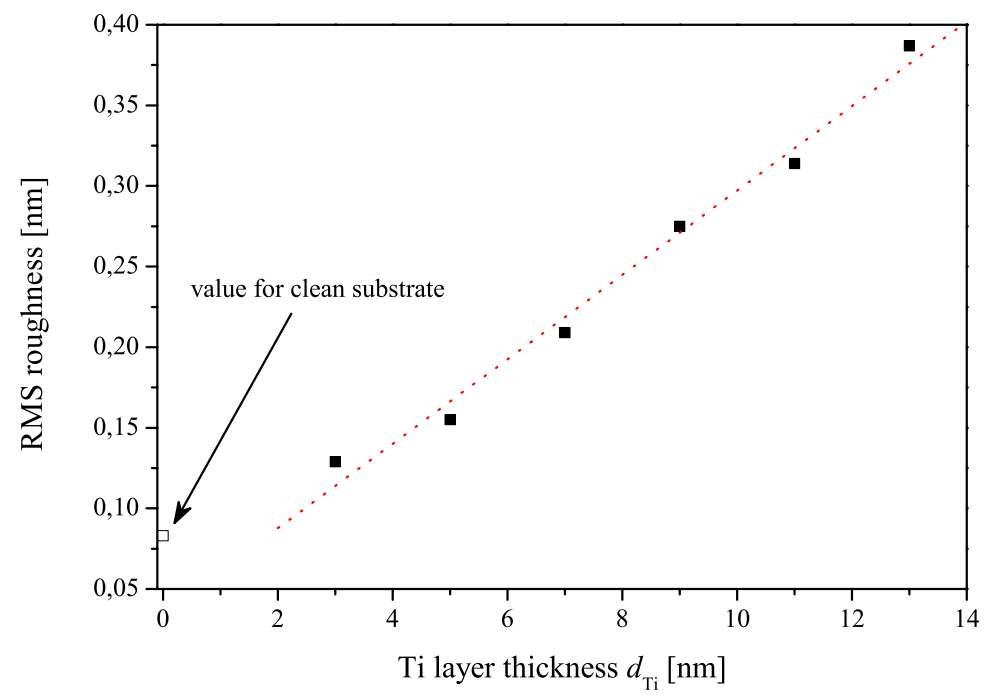

Figure 4.2: $R M S$ roughness as a function of Ti thickness for a $500 \mathrm{~nm} \cdot 500 \mathrm{~nm}$ AFM scan field. The red line is just a guide to the eyes.

According to this, figure 4.3 depicts the surface topographies for bottom electrodes of $3 \mathrm{~nm}$ Ti with Au layers of $3 \mathrm{~nm}$ up to $17.5 \mathrm{~nm}$ thickness on top. Also here the tendency of an increasing RMS roughness with increasing Au thickness can be identified (figure 4.4), even though the values for 12.5 and $17.5 \mathrm{~nm} \mathrm{Au}$ deviate from a strictly linear behavior. This might be due to a tip artefact, which can seriously distort the AFM image, as it is also indicated by the two corresponding topography images, which are somewhat blurred. However, regarding surface roughness, it is definitely advantageous to deposit bottom electrodes, which are as thin as possible.

There are a few more parameters that have to be taken into account when determining the ideal bottom electrode thickness. One of them is the correct dose for the lithography process: the dose required per unit volume of photoresist for optimum resolution can be assumed to be constant, but the physics of the exposure process may affect the dose actually received. For example the atomic number of the material beneath the photoresist determines the amount of backscattered electrons during exposure, i.e. materials with a low atomic number such as $\mathrm{Ti}$ or Si give much less backscatter 

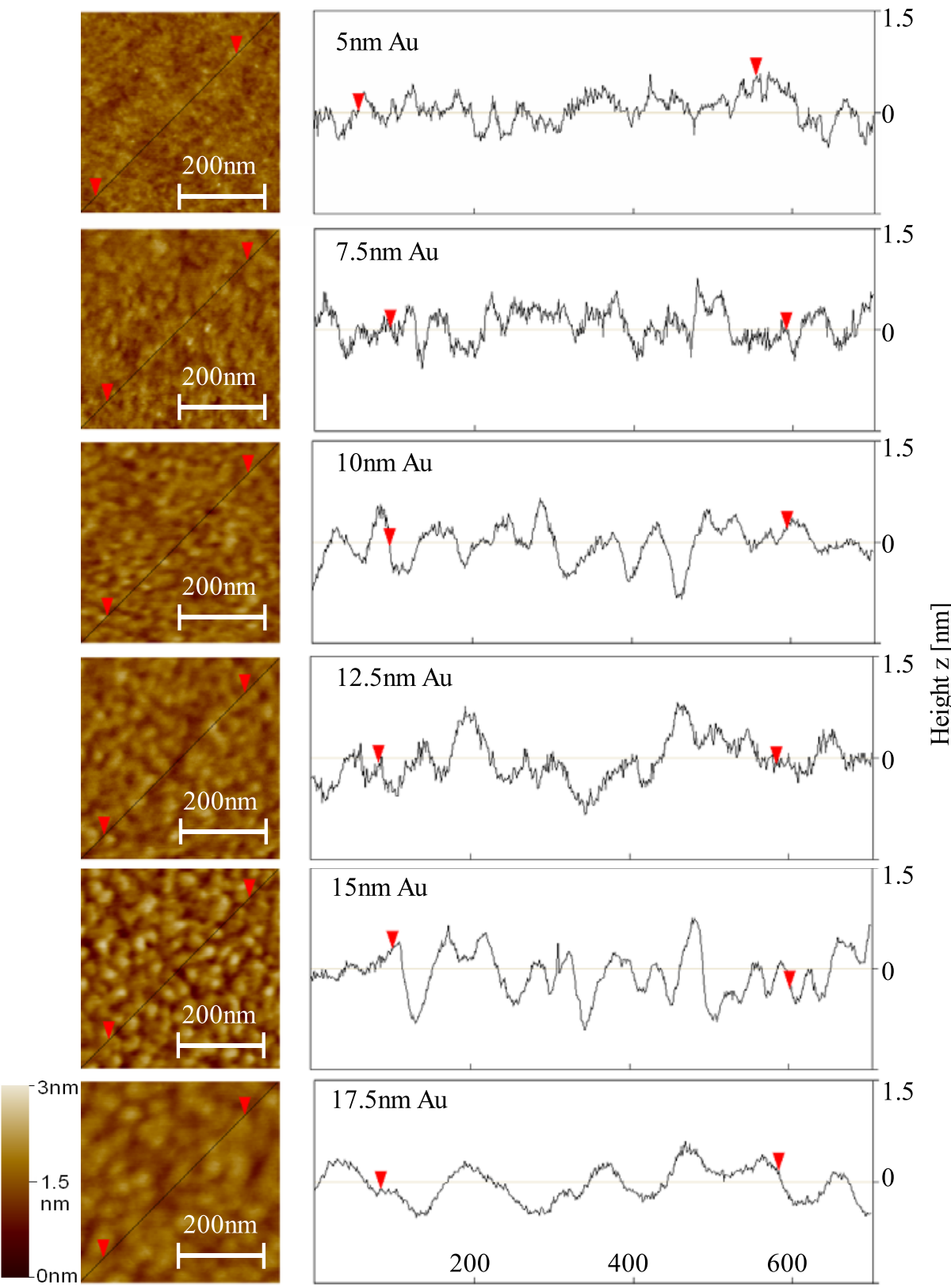

Position on sectional line $[\mathrm{nm}]$

Figure 4.3: AFM measurements on Ti/Au samples of different Au thicknesses. RMS roughness and a sectional line were analyzed for each sample.

than heavier atoms such as $\mathrm{Au}$. Hence, the optimum dosage is expected to vary not only with the bottom electrode material, but also with the total bottom layer thickness. This is confirmed by the lithography results depicted in figure 4.5. Here $\mathrm{Cu}$ dots of $20 \mathrm{~nm}$ thickness were patterned (lift-off technique) with varying dot doses between 0.0375 and $0.375 \mathrm{pAs}$ on $\mathrm{Ti} / \mathrm{Au}$ bottom electrodes with different $\mathrm{Au}$ thicknesses. The SEM images of the 


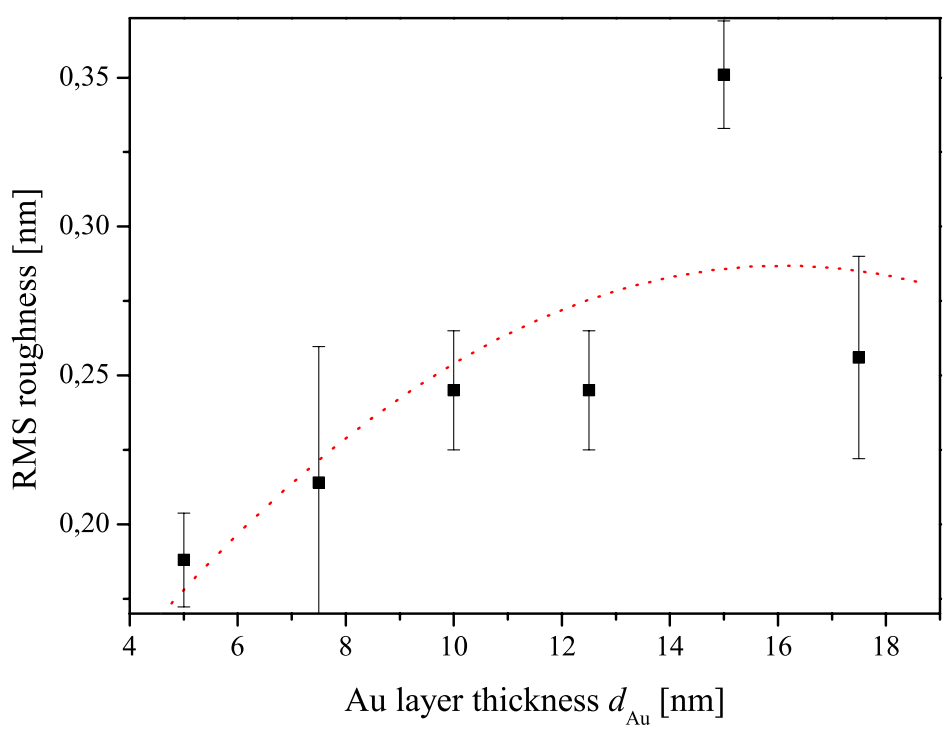

Figure 4.4: RMS roughness with standard deviation as a function of $A u$ thickness for a 500nm.500nm AFM scan field. The red line is just a guide to the eyes.

$\mathrm{Cu}$ dots after the lift-off process show that increasing the dot dose does not only increase the diameter of the dot structure, but it also results in some overexposed areas within the dots: in these regions the positive resist locally becomes a negative resist (cf. section 3.1.1), and hence the overexposed PMMA remains in these areas after the development process. Thus the $\mathrm{Cu}$ is deposited on top of the resist in these areas and stays there even after the lift-off, which results in a higher contrast (white patterns) in the SEM pictures. The SEM images on which such an effect can be observed are marked by red frames in figure 4.5, whereas black frames indicate a set of appropriate dose and thickness parameters. This detailed representation demonstrates that overexposure occurs for much lower doses, if the bottom electrode is relatively thick, i.e. many electrons are backscattered and thus enhance the effective dot dose during exposure. On the other hand the backscattered electrons can be beneficial to the fabrication of nanostructures with extremely steep side walls, which will be shown in the following section.

Last but not least the original function of the bottom electrodes has to be taken into consideration: the bottom electrodes have to carry the current during the transport measurements, which implies that they should have a reasonable low resistance compared to the one of the actual pillar device. Otherwise the applied AC (and DC) voltage would essentially drop over the electrical contacts instead over the nanopillar structure, which would 


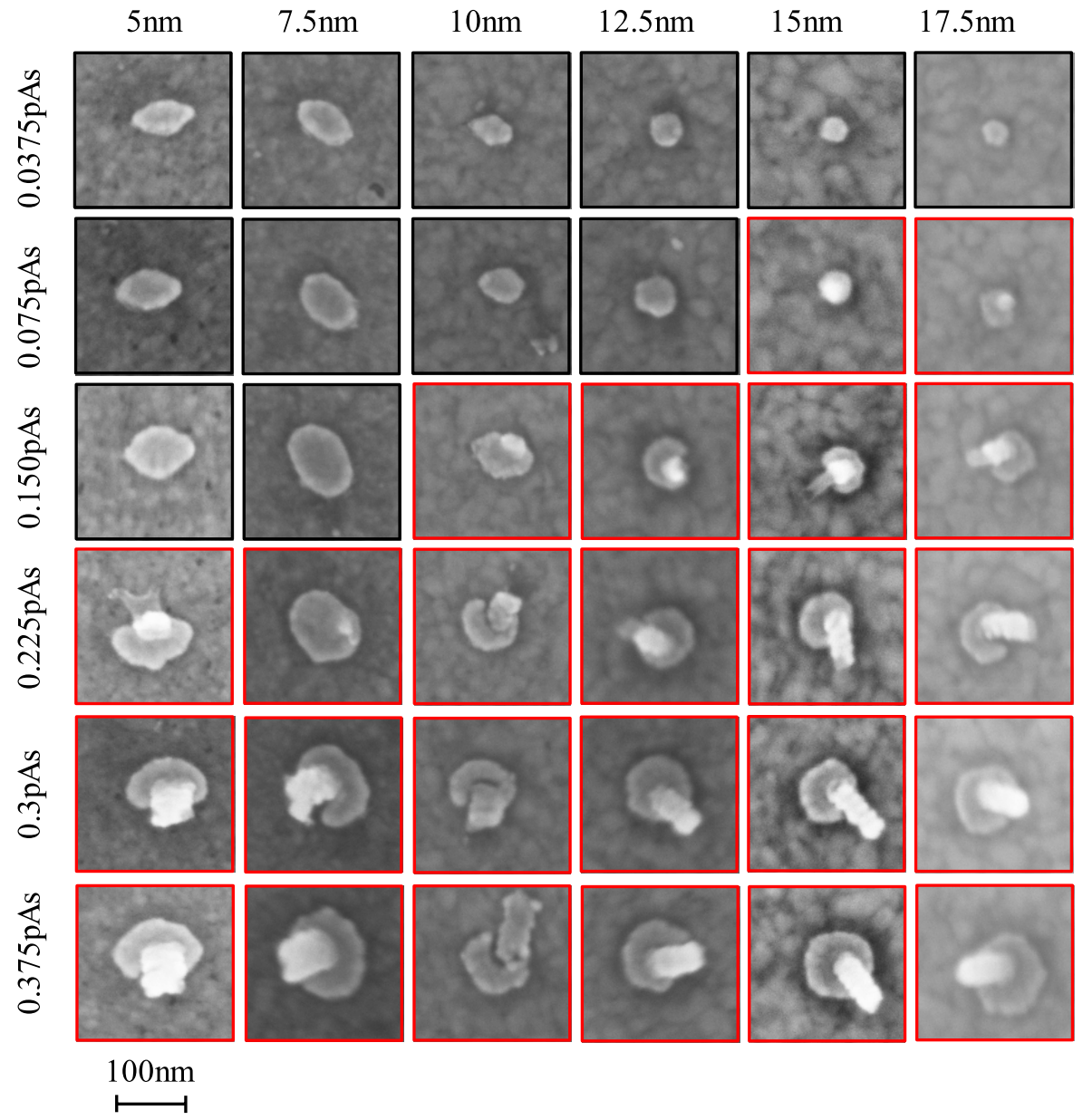

Figure 4.5: Exposure dose and Au thickness for 3nmTi/xnmAu samples.

eventually result in very small signals and hence in a deteriorated signal-tonoise ratio for the differential resistance measurements. Since the electrical resistivity of a thin film increases strongly with decreasing film thickness due to enhanced electron scattering at the film surface [61], the Au layer of the bottom contact should be sufficiently thick. Figure 4.6, which presents the resistivity data as a function of Au layer thickness measured in a four-point geometry, supports this: for Au thicknesses below $7.5 \mathrm{~nm}$ the resistivity is clearly above $200 \Omega$, which exceeds the pillar resistance of approximately $1 \Omega$ (see chapter 5) by far.

\subsection{Cross-Sectional Views of the Nanopillars: SEM Results}

The results of the sample fabrication process and their reproducibility have to be controlled by suitable analytical techniques. Cleaving samples with 


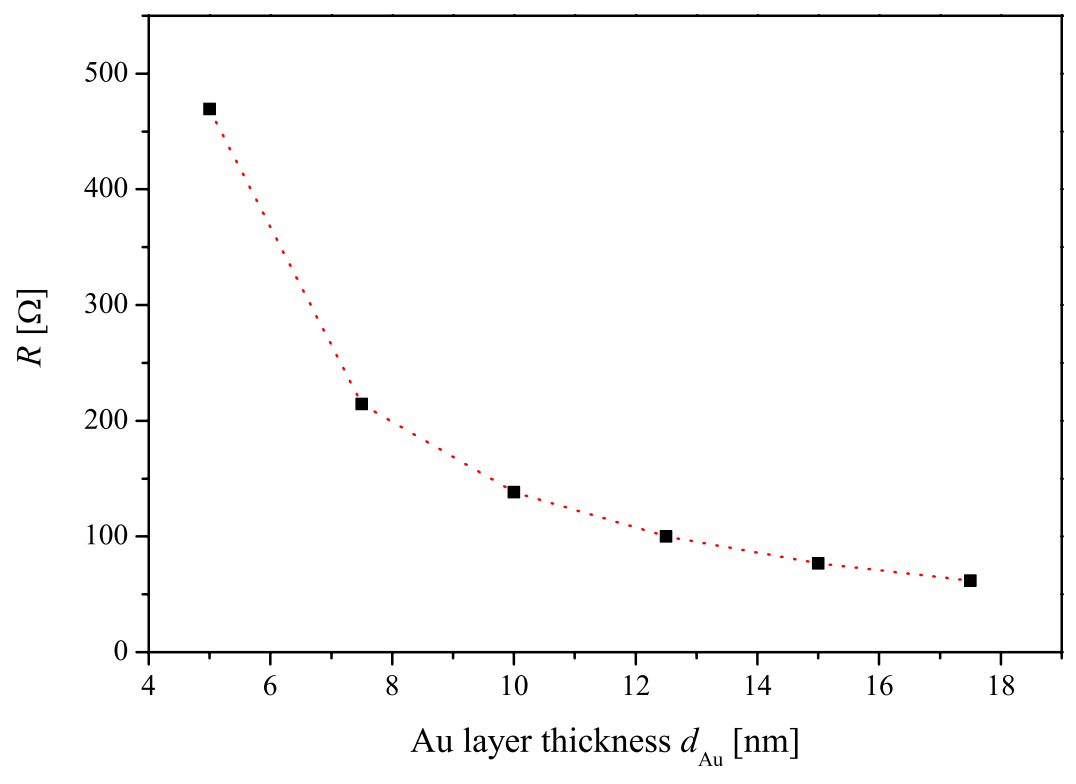

Figure 4.6: Resistance of the bottom electrode as a function of its Au layer thickness.

several pillar structures and analyzing the cross section with a SEM provides some first insight into the final geometry of the nanopillars. For this reason the corresponding preparatory process and its results will be shortly discussed within this section.

In a first step an array of nanopillars is patterned on the $\mathrm{Ti} / \mathrm{Au}$ layers deposited onto a $\mathrm{SiO}_{x}$ substrate. The array consists of approximately 100.500 dots with $2 \mu \mathrm{m}$ distance in between; hence an area of $200000 \mu \mathrm{m}^{2}$ is covered. Top views on parts of this area are presented in figures 4.7 (a) and (b). Then the substrate is scored with a diamond cutter along a section of the predetermined cleavage line, which leads through the patterned sample region. After the actual cleaving process the sample is mounted on a SEM sample holder with its cleaved cross-section facing upwards, so that this region can be directly probed. At best a few unharmed nanopillars are uncovered at the surface of the cross-section; two results of a successful cleavage are depicted in figures 4.7 (c) and (d), where cross-sectional views of a nanopillar are shown at a magnification of $180000 x$.

These images show that the diameter of the pillars is well below 100nm. Variations of $\pm 10 \mathrm{~nm}$ for the same fabrication parameters can be observed, but with this technique it is difficult to decide whether this corresponds to an effective thickness variation: the diameter might also just appear to be smaller due to a cleavage section which does not go directly through 

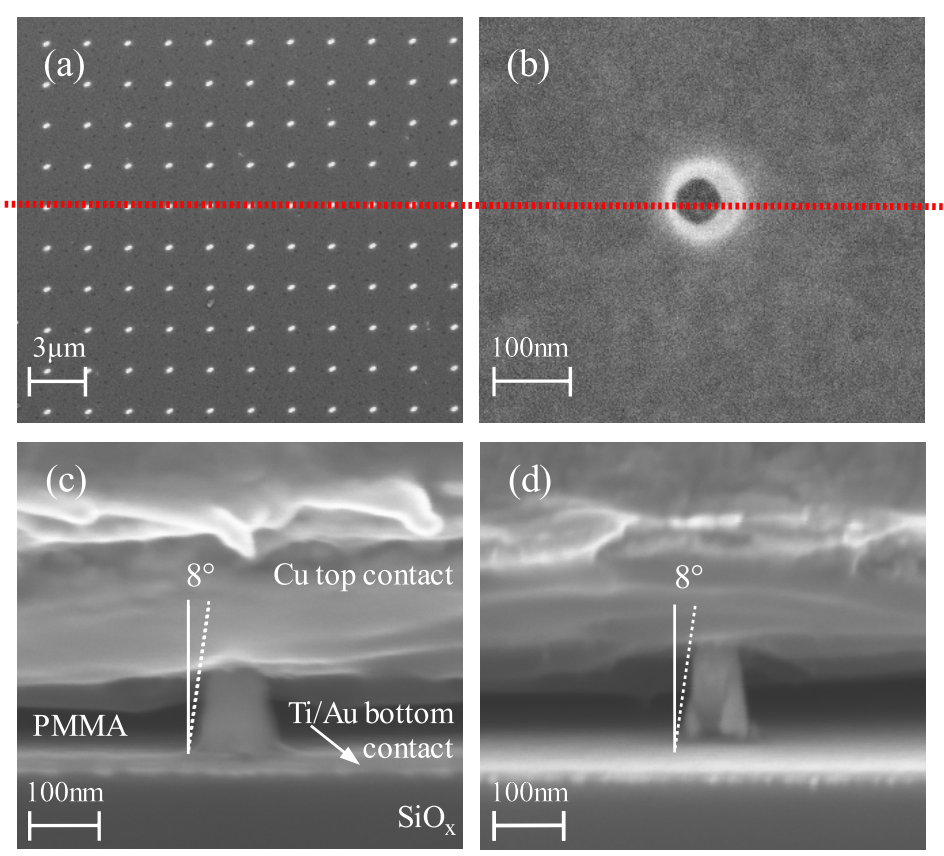

Figure 4.7: SEM images (a) top view on an array of nanopillars (b) top view on a single nanopillar (magnification 200000x) with the red line indicating the ideal line of cleavage (c) cross-sectional view after cleavage of a nanopillar with a diameter of 90nm (magnification 180000x) (d) cross-sectional view of a nanopillar with a diameter of 80nm (magnification 180000x). Due to the cleaving process, the PMMA template and the Cu top contact are slightly elastically deformed.

the middle of the nanopillar, but rather separated the pillar close to its outer edge. Comparing several top views as the one depicted in figure 4.7 (b) reveals, that the diameter of the pillars is rather constant for fixed parameter sets during the preparation process.

Another important feature is the definition of the edges. Since any kind of thermal deposition process is highly directional, the side walls of the nanopillars are expected to be quite steep. This is confirmed by the SEM images in figure 4.7 (c) and (d), which both display side wall angles of approximately $8^{\circ}$. Hence, the effective area of the pillars is accurately defined, and the multilayers prepared can be assumed to have a well-defined layer structure. The latter point will be studied further within the next section, where the results of the TEM analysis are presented.

\subsection{Further Structural Analysis of the Nanopillars: TEM Results}

TEM analysis of the fabricated nanopillars permits to study the element distribution and crystalline growth with extremely high resolution. The 
TEM lamellas were prepared by the FIB technique, which has already been described in section 3.2.4.

(a)

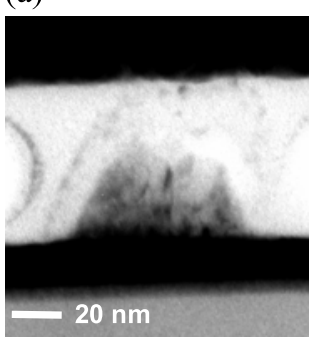

(c)

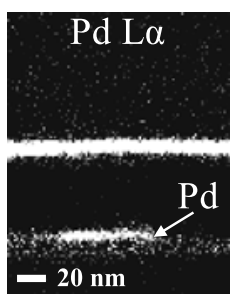

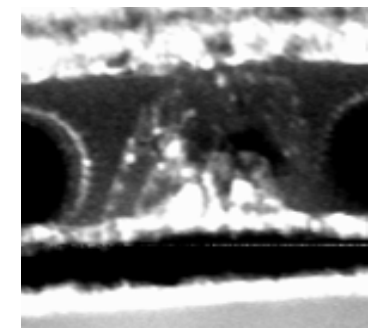

$\mathrm{Cu} \mathrm{Ka}$

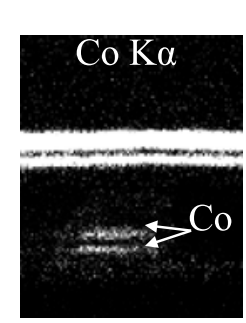

(b)

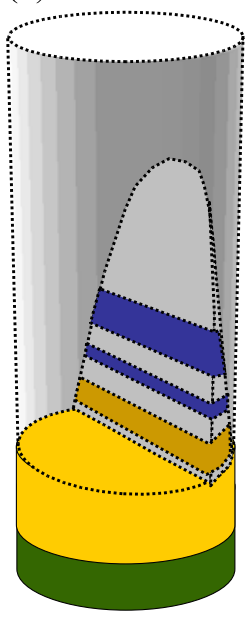

Figure 4.8: TEM images of a Co/Cu/Co trilayer nanopillar: (a) transmission and dark field images (b) corresponding schematic drawing (c) element distribution analyzed by energy dispersive $X$-ray (EDX) analysis.

A bright and a dark field image of a $3 \mathrm{nmCu} / 5 \mathrm{nmPd} / 5 \mathrm{nmCu} / 3 \mathrm{nmCo} /-$ $10 \mathrm{nmCu} / 12 \mathrm{nmCo} / 30 \mathrm{nmCu}$ trilayer nanopillar ${ }^{1}$ are displayed in figure 4.8 (a). In between the $\mathrm{Ti} / \mathrm{Au}$ bottom contact and the $\mathrm{Cu}$ top contact a conic section of the nanopillar is clearly visible. This profile results from the wedge-like cut performed by the ion beam during the FIB preparation process. Thus only a fraction of the structure can be analyzed, which also explains the reduced pillar diameter of approximately $60 \mathrm{~nm}$. A schematic illustration of the corresponding geometry can be found in figure 4.8 (b). The photoresist, which exhibits only a weak contrast compared to the one of vacuum, can be identified around the nanopillar. It is eroded by the $\mathrm{Ga}^{+}$beam, hence forming characteristic holes observed to the left and the right side of the nanopillar. Due to the background of the amorphous resist, the composition of the pillar cannot be resolved with atomic resolution in this image series. Nonetheless, the TEM data provides some profound information on the structure. The diffraction contrast in the dark field STEM-image is ascribed to strain fields, small crystallites of different orientations and planar faults like twin boundaries (right panel of figure 4.8 (a)). The crystalline growth itself seems to be coherent over the interfaces

\footnotetext{
${ }^{1}$ In this specific layer stack an extra Pd layer is present, but this does not correspond to the original trilayer geometry used for the transport measurements presented later on.
} 
of the layer stack, because the contrast in the bright as well as in the dark field images does not change from one layer to the other. The profile of the separate layers can be identified from X-ray maps obtained in the STEM mode of the electron microscope with a probe size of about $1 \mathrm{~nm}$. The corresponding images, which are depicted in figure 4.8 (c), demonstrate that the trilayer stack of the nanopillar is well-defined and no shadowing effects are observed. Besides, an overlap of the Co layers can be excluded. Due to the conical shape of the cross-section through the pillar, the image contrast is getting weaker for the layers in the upper part of the pillar until it finally fades out. On top of the PMMA the entire layer sequence is repeated.

\subsection{The Magnetic Configuration within the Nanopillars}

The magnetic configuration within the nanopillars is the key for understanding and controlling magnetic switching processes precisely. For this reason the magnetization patterns of $10 \mathrm{~nm}$ thick Co cylinders were investigated by magnetic photoemission electron microscopy (PEEM). The results are compared to micromagnetic simulations in order to deduce some important information on the dependence of the microscopic magnetic state on the thickness and the diameter of the Co cylinders.

\subsubsection{Magnetic PEEM Experiments}

The zero-field ferromagnetic nanopillars with $3 \mathrm{nmCu} / 5 \mathrm{nmPd} / 5 \mathrm{nmCu} /-$ $10 \mathrm{nmCo} / 2 \mathrm{nmCu}$ were investigated experimentally by means of the XMCDPEEM technique (cf. section 3.2.6). The samples consist of arrays of pillar structures with the same Co thickness $h=10 \mathrm{~nm}$, but different pillar diameters: $d=1000 \mathrm{~nm}, 500 \mathrm{~nm}, 200 \mathrm{~nm}$ and $80 \mathrm{~nm}$, respectively. The spacing between the nanopillars is sufficiently large in order to exclude any significant stray field coupling.

The best lateral resolution is obtained by recording a series of up to 10 images for each photon helicity. Then the images for each helicity are averaged and postprocessed with the software 'Image Pro Plus', i.e. careful drift corrections are performed. Only thereafter the asymmetry image is generated. The final image for a nanopillar with a diameter of $1000 \mathrm{~nm}$ acquired at room temperature at the L3 edge of Co (corresponding to a photon energy of $778 \mathrm{eV}$ ) is shown in figure 4.9 (b). The graytone in this image is proportional to the projection of the local magnetization direction on the incident beam. Hence, a magnetic vortex configuration with its core directly in the middle of the cylinder can be clearly identified in figure 4.9 (b). Similar vortex structures were observed in further Co nanopillars - also for structures with smaller diameters of $500 \mathrm{~nm}$ and $200 \mathrm{~nm}$ as depicted in figure 4.9 (c) and (d), respectively.

The resolution of the XMCD-PEEM is limited to $20-30 \mathrm{~nm}$ by the chromatic and spherical aberrations due to the accelerating field of the lenses, as well as by the diffraction of the aperture. This makes the investigation 

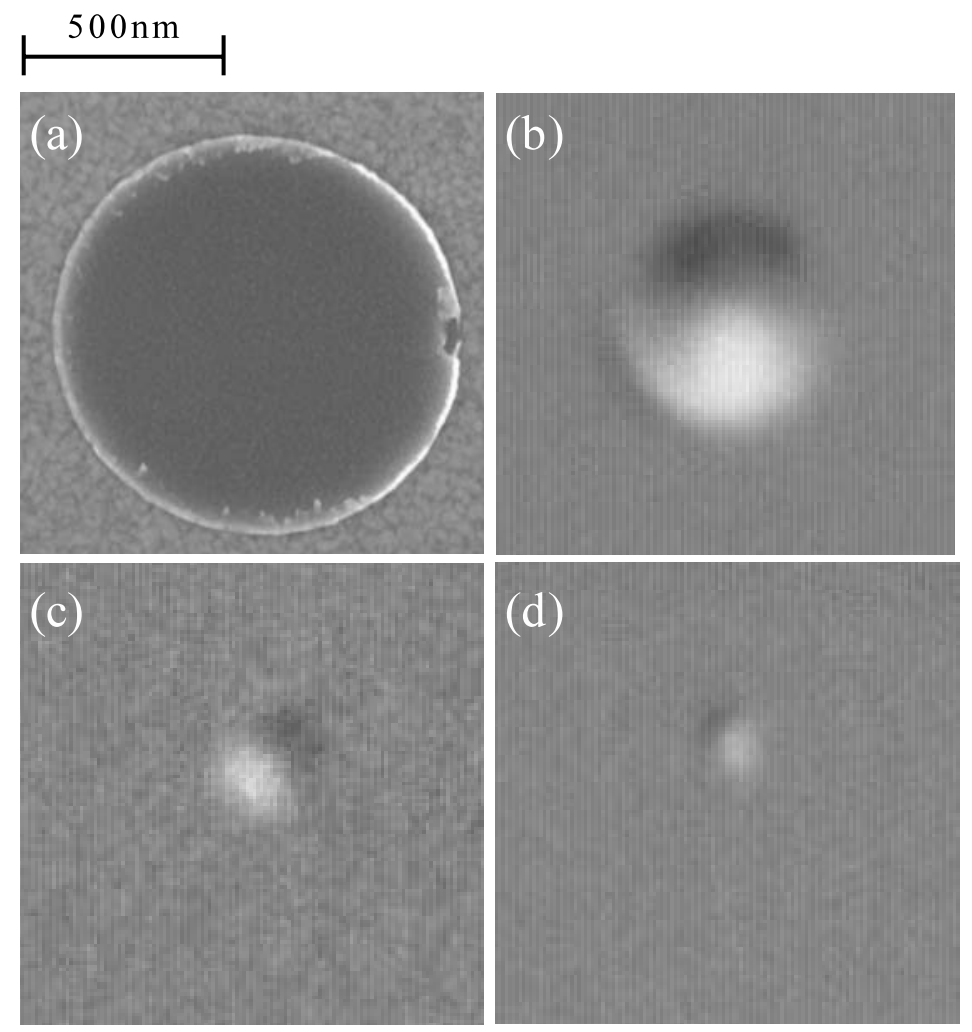

Figure 4.9: (a) SEM image of a 1000nm wide nanopillar with 10nm of Co (b) XMCD-PEEM image of an identical structure (c) XMCD-PEEM image of a 500nm wide nanopillar with 10nm of Co (d) XMCD-PEEM image of a 200nm wide nanopillar with $10 \mathrm{~nm}$ of Co.
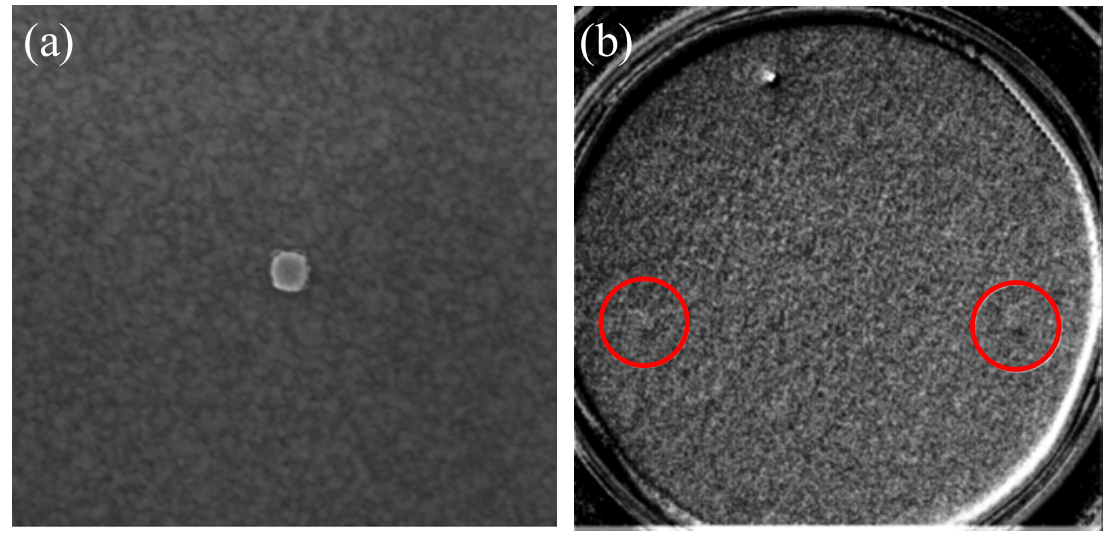

$100 \mathrm{~nm}$

Figure 4.10: (a) SEM image of an 80nm wide nanopillar with 10nm of Co (b) XMCD-PEEM image of two identical structures in the middle of the red circles. The white spot at the upper part of the image is only an artefact. 
of the most interesting nanopillars, namely of the ones with a diameter of only $80 \mathrm{~nm}$, a very challenging task.

Still, several nanopillars of these small dimensions have been probed. The two structures depicted in the right panel of figure 4.10 might show a darker contrast on the lower edge than on the upper edge, thus indicating a magnetic vortex structure. On the other hand the resolution does not really allow for the definite exclusion of a single domain state. Hence, an unambiguous conclusion about the magnetic state of the $80 \mathrm{~nm}$ wide nanopillars cannot be drawn from these experiments.

\subsubsection{Micromagnetic Simulations}

The software package 'Object Oriented Micro Magnetic Framework', which is a project of the Mathematical and Computational Sciences Division at the National Institute of Standards and Technology, was used to model the magnetic domain structure of the Co cylinders within the nanopillars. OOMMF is an open source code and freely available on the internet [62].

Here only the two-dimensional micromagnetic computation engine mmSolve2D has been applied. For this application the flat Co cylinders are divided into $N \times N$ cells onto a two-dimensional grid. Each of these cells has a quadratic cross-section of the length $l$, while its height is equal to the total thickness of the cylinder $h$. Then there is a three-dimensional vector positioned at the center of each cell, which corresponds to the saturation magnetization $M_{S}$.

After a proper definition of the micromagnetic problem, the effective field

$$
H_{\text {eff }}=H_{a n i}+H_{Z e e m a n}+H_{e x}+H_{\text {demag }}
$$

is calculated for all cells with a given initial direction of the pointwise magnetization $M$. For this the crystalline anisotropy $E_{a n i}$ and the Zeeman energy $E_{Z e e m a n}$ are computed by assuming a constant magnetization in each cell. The exchange energy $E_{e x}$ is calculated by means of an eight-neighbor bilinear interpolation as it has been introduced in [64]. Since for the calculation of the demagnetization energy $E_{\text {demag }}$ the interaction of each cell with all others has to be taken into account, this step requires the largest computing power. Hence, a fast Fourier transform (FFT) technique is employed. For this purpose different routines representing different interpretations of the discrete magnetization are available; for this thesis the model ConstMag was used.

Then the Landau-Lifshitz equation

$$
\frac{d}{d t} \vec{M}=-\gamma \vec{M} \times \vec{H}_{e f f}-\frac{\gamma \alpha}{M_{S}} \vec{M} \times\left(\vec{M} \times \vec{H}_{e f f}\right),
$$

which is equivalent to equation 2.17, is integrated numerically by means of a predictor-corrector technique. For each iteration step the terms on the right hand side of equation 4.2 are extrapolated from the previous and the current value in a linear manner. Then they are integrated over a time interval $\Delta t$, thus yielding a quadratic prediction for the magnetization $\vec{M}$ at 
the next time step. This predicted value for $\vec{M}$ is used for a new evaluation of the right hand side of equation 4.2 , and thus $d \vec{M} / d t$ can be recomputed. The resulting value is then combined with the value at the current step to produce a linear interpolation of $d \vec{M} / d t$ over the new interval. Eventually, this result for $d \vec{M} / d t$ is integrated to receive a final estimation for $\vec{M}$ at the new step.

The complete iteration step described in the previous paragraph is accepted only if the total energy is decreased for the new value of $\vec{M}$. Besides, the maximum error between the predicted and the final estimation for $\vec{M}$ has to fall below a certain value. Otherwise, the step is rejected and the entire procedure is repeated for a smaller time interval $\Delta t$. This routine is continued, until a certain stopping criterion is fulfilled. For the simulations performed here, this stopping criterion is given by the maximum value of $\left|M \times H_{e f f}\right| / M_{S}^{2}$ dropping below the specified control point torque value of $10^{-5}$.

Further input parameters are the saturation magnetization $M_{S}=1400$. $10^{3} \mathrm{~A} / \mathrm{m}$, the exchange constant $A=30 \cdot 10^{-12} \mathrm{~J} / \mathrm{m}$ and the anisotropy constant $K_{1}=5.2 \cdot 10^{5} \mathrm{~J} / \mathrm{m}^{3}$. The Co structure is assumed to be hcp and a Gilbert damping parameter of $\alpha=0.5$ is used. Due to [63] the length $l$ of the cells has to be smaller than half of the magnetostatic exchange length $l_{\text {ex }}=\sqrt{2 A /\left(\mu_{0} \cdot M_{S}^{2}\right)}=4.9 \mathrm{~nm}$. Thus the length of the cells is chosen to correspond to $l_{e x}=1 \mathrm{~nm}$. This value promises reasonable results with a total energy error of less than $1 \%$. Then the simulation is started for two different initial magnetization configurations within the Co cylinder: the first one is an ideal vortex state, whereas the second one assumes a totally homogeneous magnetization. The relaxation process for these states continues until the stopping criterion, which has already been defined in the previous paragraph, is reached.

Figure 4.11 displays the corresponding results for the total energy as a function of the thickness of the Co cylinder. The simulation was not only performed for two different initial magnetization states (filled squares represent the vortex state; open circles denote the homogeneous state), but also for two different nanopillar diameters $d$ of 100nm (red symbols) and $80 \mathrm{~nm}$ (blue symbols), respectively. As expected the homogeneous magnetization is energetically favored for thinner samples. The vortex structure with the reduced stray field but increased exchange energy is found only above a critical thickness $h_{c}$. This critical thickness amounts to approximately $7 \mathrm{~nm}$ for a $100 \mathrm{~nm}$ wide Co cylinder, and it increases significantly for smaller structure sizes: $h_{c}=9.5 \mathrm{~nm}$ for a cylinder with a diameter of $80 \mathrm{~nm}$. Hence, the ground state in zero external field is the homogeneous magnetization configuration for thin Co cylinders, and a vortex state for thicker Co layers. In between these two stable states there is an energy barrier, but for Co cylinders with thicknesses close to the critical value $h_{c}$ and for sufficient temperatures, thermally induced switching between these two states seems to be thoroughly possible.

Figure 4.12 shows the results of a second OOMMF simulation, in which 


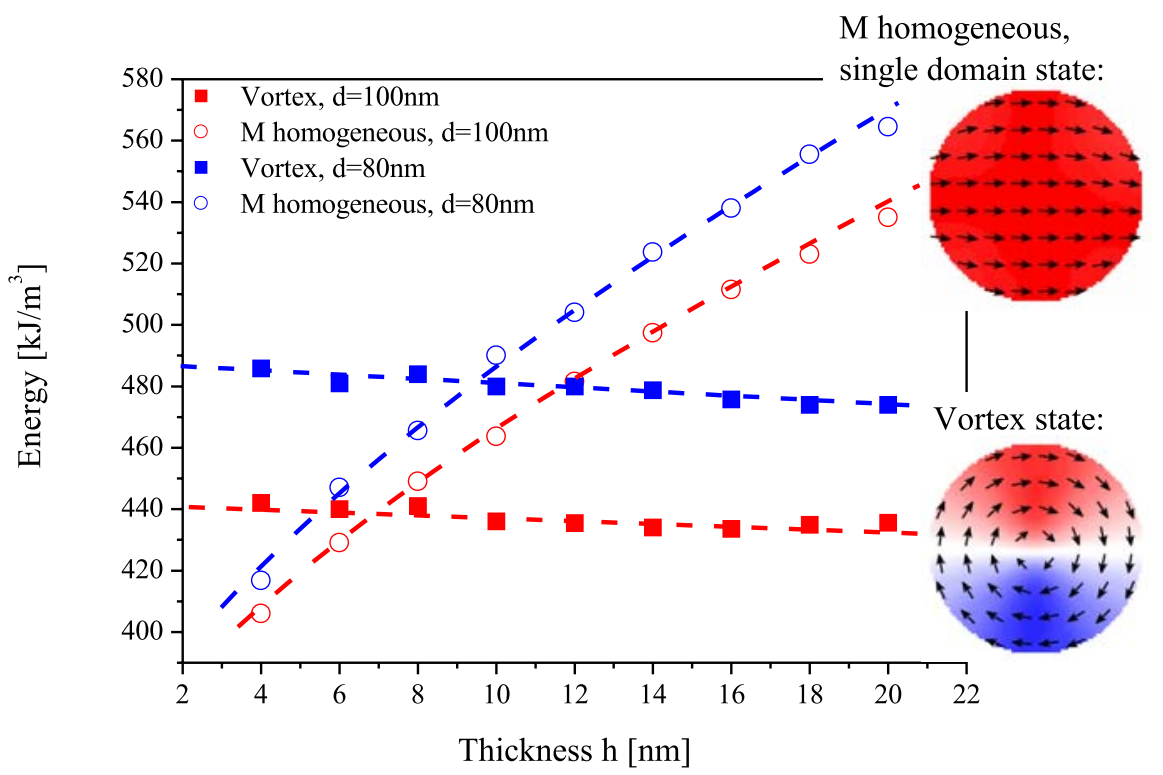

Figure 4.11: Micromagnetic simulation for the determination of the magnetic configuration (at zero external field) within a Co cylinder with thick$n e s s ~ h$ and a diameter of $80 \mathrm{~nm}$ and $100 \mathrm{~nm}$, respectively. The insets at the right side show the corresponding magnetization configuration in the plane of the Co layer.

the development of an initial vortex configuration within a Co cylinder in higher external fields has been investigated. The Co layer is $80 \mathrm{~nm}$ wide and $12 \mathrm{~nm}$ thick; all other input parameters correspond to the ones listed above. While the red curve marks the out-of-plane magnetization $M_{z}$ as a function of the external field pointing along the $z$ direction (out of plane), the blue hysteresis loop displays the in-plane magnetization $M_{x}$ in dependence on the external field directed along the $x$ direction.

For increasing in-plane fields the $M-H$ loop (blue) shows the expected behavior: the magnetization within the Co layer rapidly points into the direction of the external field, and the saturation magnetization is reached at a very low value of $\mu_{0} H=+0.13 \mathrm{~T}$. When reversing the field direction the vortex state does not reappear at the low field regime, but the simulation reveals that the magnetization turns out of plane in order to switch over to the saturated single domain state at $\mu_{0} H=-0.20 \mathrm{~T}$.

The hysteresis loop for out-of-plane fields (red curve) looks significantly different: here the starting configuration is a vortex with its core pointing towards the $-z$ direction. For increasing positive field values the magnetization then tilts subsequently out of plane towards $+z$, but the vortex core initially remains in the opposite direction. Only at $\mu_{0} H=+1.01 \mathrm{~T}$ the core flips and is aligned with the external field (consequently the helicity of the 


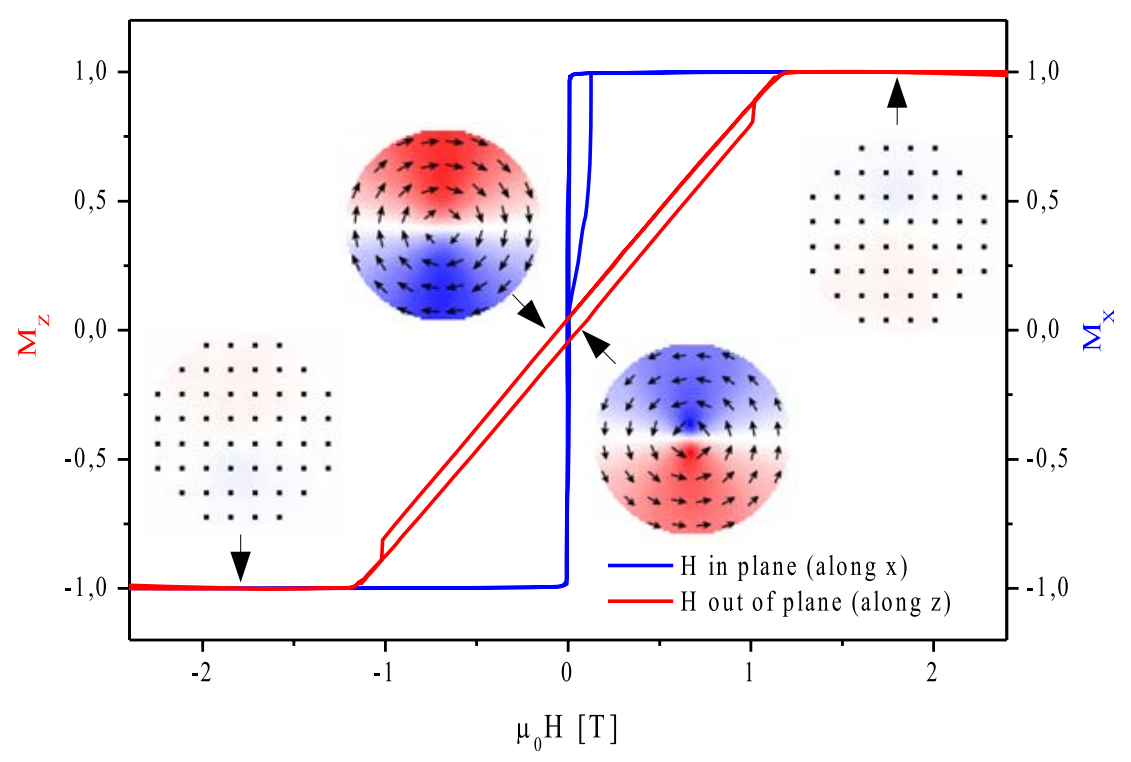

Figure 4.12: Micromagnetic simulation for the determination of the magnetic configuration within a Co cylinder with a thickness of 12nm and a diameter of 80nm in different external fields. The red curve corresponds to an out-of-plane magnetic field, whereas the blue one depicts the field in plane geometry. The insets show the corresponding magnetization configuration in the plane of the Co layer for the out-of-plane fields.

vortex switches as well). The saturation field amounts to $\mu_{0} H=+1.21$ T. Upon decreasing the field again, the magnetization slowly turns back towards the vortex configuration. For negative values of $H$ the magnetization then tilts more and more in the $-z$ direction, but again the vortex core points opposite to the external field. It switches to parallel alignment at $\mu_{0} H=-1.01 \mathrm{~T}$ and $M_{z}$ further increases until $\mu_{0} H=-1.21 \mathrm{~T}$.

\subsection{Discussion}

Prior to the preparation of the actual nanopillars the bottom contacts have been characterized by AFM and resistance measurements: electrodes with $3 \mathrm{~nm} \mathrm{Ti}$ and $7.5-10 \mathrm{~nm} \mathrm{Au}$ are found to possess a relatively low surface roughness in combination with reasonable low resistivity values. Hence, parameters within this range were chosen for the sample fabrication process. The structural characterization of the finished samples by means of cross-sectional SEM images as well as by TEM analysis shows that the simplified one-step fabrication process, which has been developed within this thesis, yields accurate pillar structures with relatively steep sidewalls. The corresponding EDX investigations indicate a stack of well-defined layers. 
The diameters of the nanopillars are found to be approximately 80nm. Due to these small dimensions, the magnetic characterization turns out to be very complicated: here the XMCD-PEEM experiments do not really allow for a definite identification of either a vortex or a totally homogeneously magnetized state. Thus micromagnetic simulations are performed in order to determine the magnetic configuration with the lowest total energy as a function of the nanopillar thickness. According to these simulations, in the absence of an external field the vortex state is stable for thicknesses above $7 \mathrm{~nm}(9.5 \mathrm{~nm})$ for structures with a diameter of $100 \mathrm{~nm}(80 \mathrm{~nm})$. For smaller thicknesses a homogeneous magnetization is found to be energetically favorable. In another context the development of the vortex state with increasing magnetic fields is simulated, and it is revealed that for out-of-plane fields the magnetization subsequently aligns with the external field. However, the saturation magnetization is only reached at field values above $1.2 \mathrm{~T}$. 


\section{CHAPTER 5}

\section{Current-Induced Excitations in Ferromagnetic Trilayer Samples}

In this chapter the experimental results for the transport measurements on the ferromagnetic trilayer samples are presented and discussed. The layer sequence of the samples corresponds to the one introduced in section 3.1.3; i.e. the relevant layers are the magnetically 'free' $3 \mathrm{~nm}$ Co layer, which is followed by a $10 \mathrm{~nm}$ thick $\mathrm{Cu}$ spacer layer, and the magnetically 'fixed' layer with $12 \mathrm{~nm}$ of Co. Several samples were analyzed, but here representative data for only two of them, which will be referred to as device A and device $\mathrm{B}$ in the following, is shown. Both current sweeps at fixed applied fields and field sweeps at fixed current bias were performed in the field in-plane and the field out-of-plane geometry for field values up to 5T. Sweeping times for the DC current were approximately $d I / d t=0.3 \mathrm{~mA} / \mathrm{s}$, whereas the field was swept at a rate of $d B / d t=4 \mathrm{mT} / \mathrm{s}$. Compared to the time regime of ps or ns, where magnetization dynamics processes are observed, these time scales are rather large. The differential resistance was measured with an excitation amplitude of $100 \mu \mathrm{A}$ at a frequency of $f=7013 \mathrm{~Hz}$. The latter value was chosen due to its good signal-to-noise ratio for the hardware configuration specified in section 3.2.5, but the phenomena observed can also be detected for other AC frequencies. All measurements were carried out at $T=10 \mathrm{~K}$.

\subsection{Current Sweeps at Fixed Applied Fields in the Field Perpendicular Geometry}

Figure 5.1 displays the results of the transport measurements for device A for DC currents between $-50 \mathrm{~mA}$ and $+50 \mathrm{~mA}$ in constant out-of plane fields up to $5 \mathrm{~T}$. For clarity the $d V / d I(I)$ curves of different field values were shifted vertically by $8 \mathrm{~m} \Omega$. Current sweeps from negative bias to positive 
bias are indicated by blue lines, whereas the opposite sweep direction is marked in green.

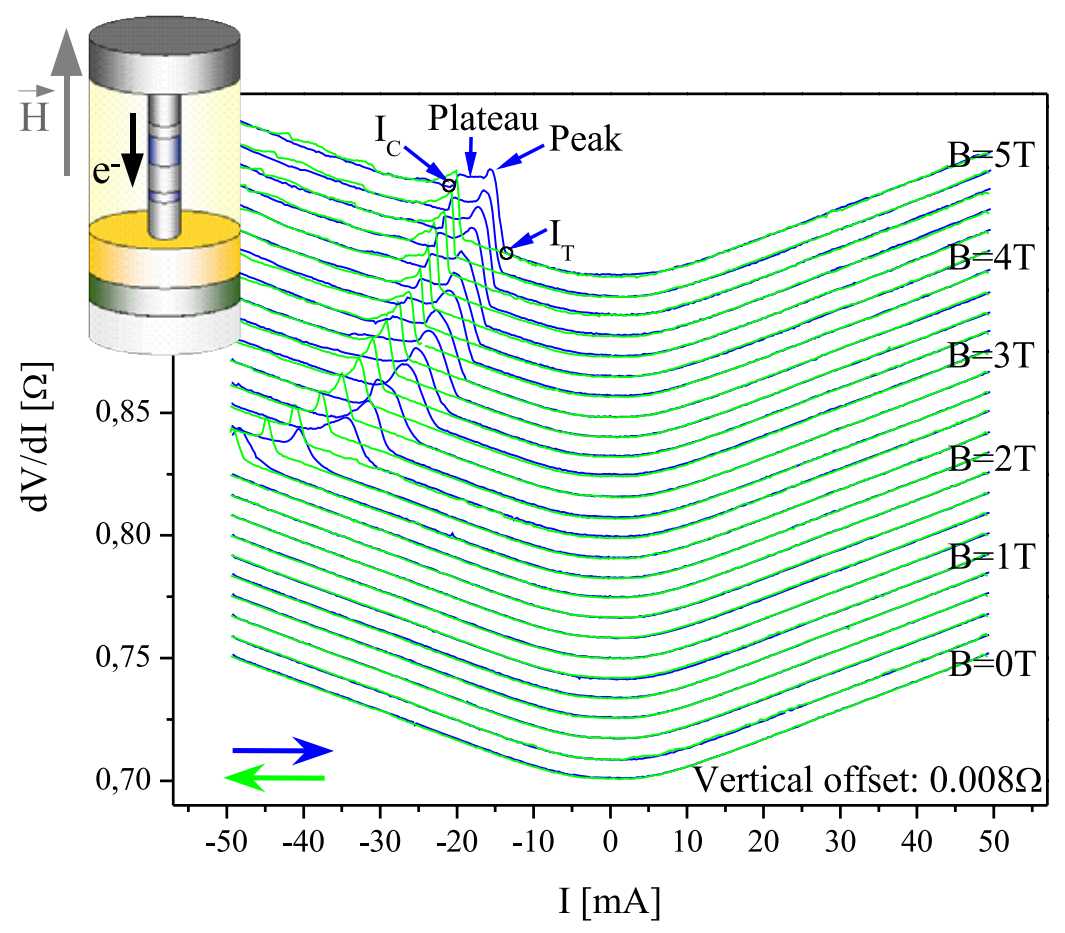

Figure 5.1: Current sweeps for a 3nmCo/10nmCu/12nmCo trilayer nanopillar (device A) in the field perpendicular to the plane geometry. The curves for different magnetic field values are shifted vertically by $8 \mathrm{~m} \Omega$. The inset displays the direction of positive current flow and the corresponding magnetic field direction.

In the following the general observations for the measurements on devices $\mathrm{A}$ and $\mathrm{B}$ are pointed out and an overview is given. A detailed discussion follows in the subsections 5.1.1 to 5.1.4.

For external field values below $1.8 \mathrm{~T}$ only a varying background resistance, which increases for higher current values, can be observed. This parabolic change in resistance is definitely independent of the applied field, but very small variations in curvature for opposite current polarities are detected. The origin of this effect and its consequences are discussed further in section 5.1.1.

In higher external fields above the demagnetization field of a thin Co film, which corresponds to a value of $\mu_{0} M_{S} \approx 1.5 \mathrm{~T}$, an abrupt increase in the differential resistance is superimposed on the varying background resistance. This change of resistance is asymmetric in current; i.e. it is observed for negative current bias only. The resistance increase is always accompanied 
by a characteristic peak structure, which appears whenever the threshold current value $I_{T}$ is exceeded. For sufficient external fields $\mu_{0} H>3.2 \mathrm{~T}$ these peaks are followed by a plateau of higher resistance with increasing current $I$, until a small downward step in $d V / d I$ can be observed. The current value after the peak structure (and after the plateau) is denoted as the critical current $I_{C}$ in the following. The resistance plateaus are most obvious for the current sweep direction from negative to positive bias (blue lines). The resistance changes described in this paragraph can be attributed to currentinduced changes in the magnetic configuration within the nanopillars, as they are described in section 5.1.2.

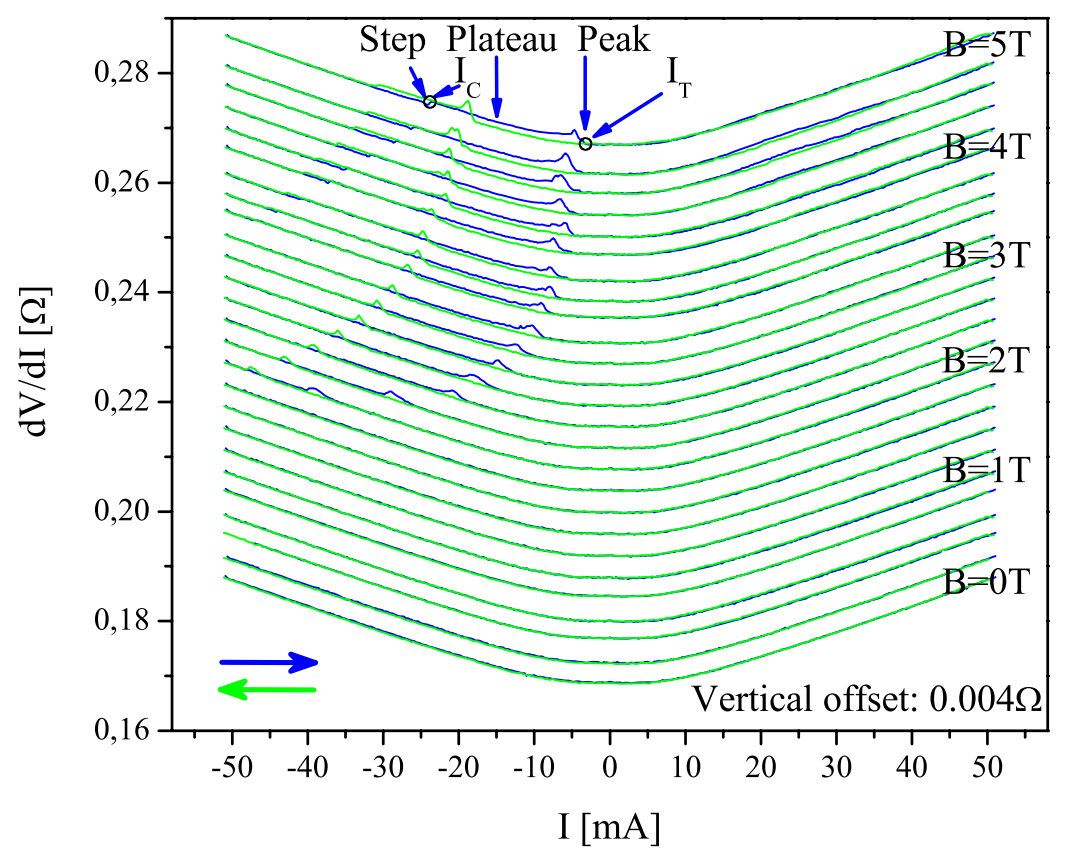

Figure 5.2: Current sweeps for a 3nmCo/10nmCu/12nmCo trilayer nanopillar (device B) in the field perpendicular to the plane geometry. The curves for different magnetic field values are shifted vertically by $4 \mathrm{~m} \Omega$.

The resistance change is hysteretic, occurring at higher absolute current values for increasing current. The origin of the observed hysteresis is discussed in section 5.1.3.

The magnitude of the resistance change described above, which means the difference between the low and the high resistance state, is found to increase slightly with larger external magnetic fields. Also the hysteresis itself shows a distinct field behavior: the width of the hysteresis increases in higher magnetic fields. Furthermore, the onset of the excitations is found 
to shift to lower threshold currents $I_{T}$ for increasing fields. All these fielddependent features are commented on in section 5.1.4.

Device B, which has been prepared in the same fabrication run as device A, essentially shows the same remarkable characteristics (see figure 5.2). However, the onset of these effects occurs at lower absolute values of the threshold current. For example, the threshold current for a sweep from negative bias to positive current bias at $B=5 \mathrm{~T}$ corresponds to $-4.5 \mathrm{~mA}$ for device B, but it amounts $-16 \mathrm{~mA}$ for device A. Furthermore, the magnitude of the observed effect is considerably smaller for device B. Also it is evident that the resistance of device B is decreased again by a downward step for threshold currents much higher than $\left|I_{T}\right|$. In contrast to device $\mathrm{A}$, where only a small decrease in resistance is observed after the characteristic plateau in really high fields, this resistance reduction in device B is relatively large. All of these effects are discussed within the following sections as well.

\subsubsection{Sample Resistance and Current-Dependent Background Resistance}

All current sweeps show a parabolic background resistance, which is independent of the applied field. This increase in resistance for larger currents is attributed to Joule heating processes, i.e. the lattice temperature rises due to the high current densities at the order of $10^{8} \mathrm{~A} / \mathrm{cm}^{2}$. The curvature of the background resistance slightly varies for opposite current polarities, because different contact resistances for the top and the bottom electrodes as well as other local asymmetries may in principle lead to varying spindependent interface resistances. This does not only make the determination of the actual size of the spin-transfer effects more complicated, but it also leads to the question, whether some observed effects could (partially) arise just because of thermal changes (in contrast to current-induced effects). However, this objection can be refuted by some rather simple arguments: first of all, the peaks and steps in $d V / d I$ show a strong dependence on the applied magnetic field, but the background resistance always appears with the same curvature for all field values. This suggests a connection between the spin-polarized current and the magnetic state of the two ferromagnetic layers, but no evidence for a direct correlation between these parameters and the lattice temperature is given. The same argument holds also true for the hysteretic effects observed. Besides, field sweeps at fixed current values (i.e. at constant lattice temperature) show similar features as the current sweeps; see section 5.3. Even the magnitudes of the observed effects are similar for both experiments, so that dominating temperature-induced effects can definitely be ruled out.

Another issue, which has already been shortly addressed above, is the exact determination of the magnitude of the observed resistance changes in spite of the superimposed parabolic background resistance. This problem can be avoided by comparing the high differential resistance at $I_{C}$ to the differential resistance at the same DC current value but at zero magnetic field, where only the low resistance state is observed. 
Comparing not only the background resistance, but also the absolute resistance values of devices $\mathrm{A}$ and $\mathrm{B}$ yields totally different results: the resistance of device A at zero bias current and zero magnetic field amounts to $0.705 \Omega$, whereas the same resistance measurements for sample B show only $0.167 \Omega$. These deviations in nanopillar resistance could either be accounted for by large variations in pillar sizes or in contact resistances. The pillar dimensions were studied carefully for various parameter sets (see sections 4.1 and 4.2), and no variations in diameter which might result in a resistance increase by a factor of four have been observed (assuming that the device resistance scales approximately linearly with its area as measured in [20], the radius should have varied by a factor of two in order to explain the observed scatter in resistance). The SEM and TEM studies presented in the previous chapter also show no indication for parallel conductance channels such as shunting effects along the edges of the nanopillars or even parasitic shorts between the top and the bottom electrodes. Nonetheless, their existence cannot be completely excluded. However, most likely different or additional contact resistances for the electrodes are responsible for the observed resistance deviations for devices $\mathrm{A}$ and $\mathrm{B}$. This makes a direct comparison between results obtained from different devices very complicated, which should be kept in mind throughout the following sections.

\subsubsection{Static Excitations versus Dynamic Excitations}

Figure 5.3 displays the differential resistance $d V / d I$ as a function of the DC bias current for device $\mathrm{A}$ in an out-of-plane field of $B=5 \mathrm{~T}$. The increase in resistance at $I_{T 1}=-13 \mathrm{~mA}$ (sweep direction from negative to positive current bias) and $I_{T 2}=-18.5 \mathrm{~mA}$ (opposite sweep direction from positive to negative current bias) is clearly visible. Comparing the high resistance value after the peak structure at $I_{C}=25 \mathrm{~mA}$ to the corresponding low resistance value at the same DC current value but at zero external field, yields a relative resistance change of $\Delta R / R=2.7 \%$.

The observed increase in resistance occurs most likely due to a magnetic state close or even identical to the antiparallel GMR configuration. This means that the magnetically 'fixed' layer is still oriented out-of-plane along the external field, whereas the magnetically 'free' $3 \mathrm{~nm}$ Co layer has been reorientated by the spin current into an antiparallel configuration. Resistance changes in the same order of magnitude (i.e. $5 \%$ and $2.2 \%$, respectively) have already been observed in conventional CPP GMR experiments for similar $\mathrm{Co} / \mathrm{Cu} / \mathrm{Co}$ structures $[66,67]$. This makes an interpretation of the resistance increase in terms of a small-angle deviation from the parallel configuration (see black line in figure 5.4) very unlikely. GMR measurements in which the external magnetic field is swept at zero current bias might give further information on this issue; the corresponding results are presented in section 5.2.

The fact that the magnetic state of the device is only deduced from resistance measurements leads also to some uncertainty about the nature of the magnetic excitations in the 'free' layer in high external fields: in earlier 


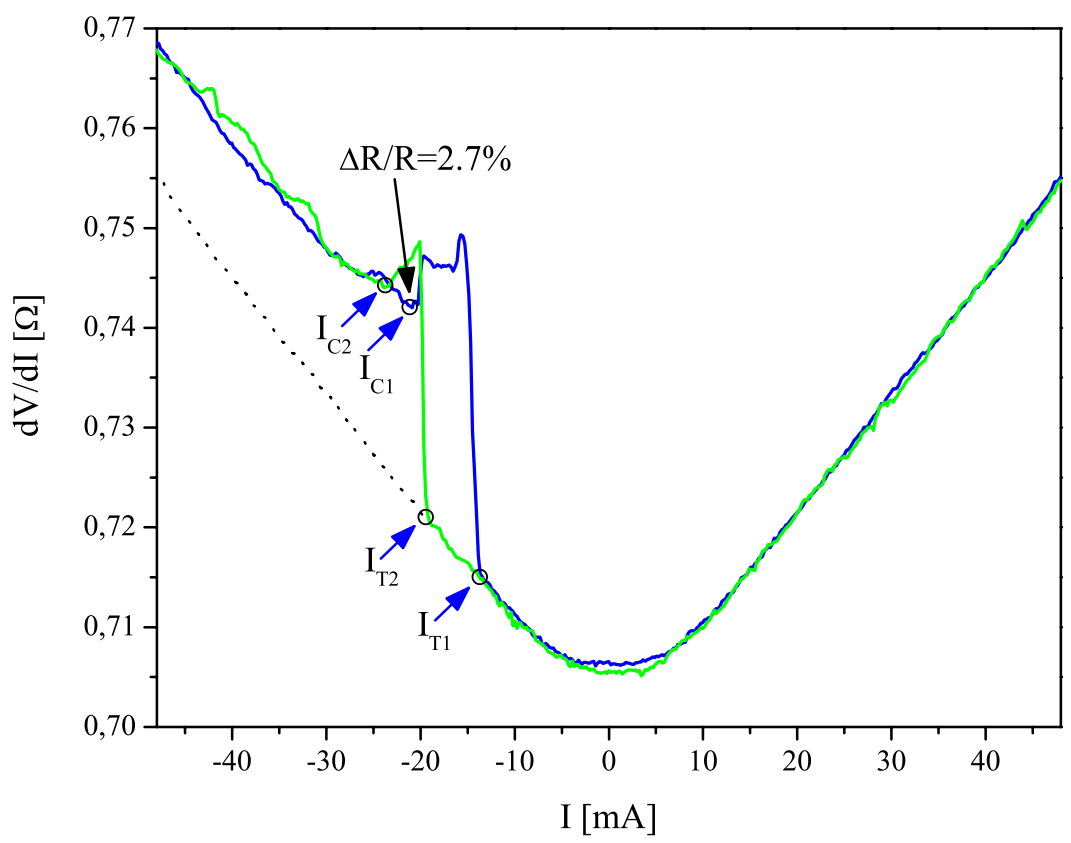

Figure 5.3: Current sweeps for a 3nmCo/10nmCu/12nmCo trilayer nanopillar (device $A$ ) in the field perpendicular to the plane geometry at $B=5 T$ and $T=10 K$.

experiments the observations of dynamic excitations in the form of magnetization precession have been reported $[3,4,65]$. On the other hand, also evidence for a current driven complete reversal of the magnetization in the free layer, i.e. a static change in the resistance state, has been found [32, 66, 69]. Özyilmaz et al. investigate similar $\mathrm{Co} / \mathrm{Cu} / \mathrm{Co}$ samples also in the field perpendicular to the plane geometry in [66]. From their experiments as well as from their micromagnetic simulations they conclude that the 'free' layer experiences an irreversible transition from parallel to antiparallel alignment marked by a peak structure for $I>I_{T}$, which characterizes the region of large amplitude dynamic excitations. For $I>I_{C}$ these large amplitude precessions result in a global magnetization reversal (cf. figure 5.4). Özyilmaz et al. point out the hysteretic change in resistance as the most obvious indication for a static antiparallel configuration, since dynamic excitations are expected to decay rapidly and hence should appear reversible on the timescale of the measurements [66]. These findings are supported by Kiselev et al. [70], who do not detect any dynamics with well-defined frequency in the microwave spectra of $\mathrm{Co} / \mathrm{Cu} / \mathrm{Py}$ nanopillar structures above $1.7 \mathrm{~T}$ except for the small current range $I_{T}<I<I_{C}$, in which the peak structure is detected in $d V / d I$. Since all curves presented in figure 5.1 show 
large hysteretic effects plus a characteristic peak structure, an interpretation equivalent to the ones given in $[66,70]$ seems to be plausible: the peak structure (and the subsequent plateau for very high fields) in the differential resistance most likely signifies a spin wave instability, which marks the switching process of the 'free' layer from a parallel to an eventually static antiparallel magnetization configuration and vice versa. However, it should be noted that crossing the small-amplitude stability boundary does not necessarily result in a magnetic reversal, because the non-linearity of large amplitude excitations can stabilize certain large amplitude orbits without necessitating a complete magnetization reversal [71]. Hence, the possibility of further dynamic excitations cannot be completely ruled out.

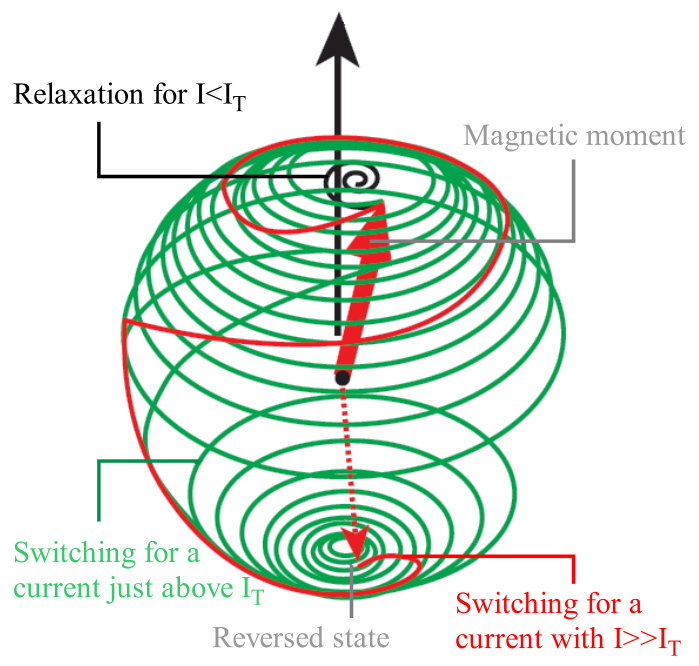

Figure 5.4: Precessional orbits for small angle excitations (black line) and large angle excitations (green and red lines). The latter ones may result in magnetic reversal. After [68].

So far only the high field regime for fields above 1.5T has been discussed. For lower magnetic fields no current-induced excitations are observed in figure 5.3. This is totally contrary to the results reported elsewhere for similar samples analyzed in the low field regime [20,70] (fields perpendicular to the plane geometry) or even without an external field [69, 72]. The absence of any excitations below the demagnetization field in device A can be explained by the special magnetic configuration within the $12 \mathrm{~nm}$ polarizing Co layer: due to its circular base area and its relatively large thickness, a magnetic vortex state is energetically favorable for this geometry in low magnetic fields. This is also supported by the micromagnetic simulations presented in section 4.4.2. Hence, as long as the external field is not strong enough to pull the magnetization of the $12 \mathrm{~nm}$ Co layer out of plane, no sufficiently spin-polarized current is generated. Only when the magnetization direction is tilted almost completely perpendicular to the sample surface, the net polarization of the current is large enough in order to produce an adequate spin torque effect. 


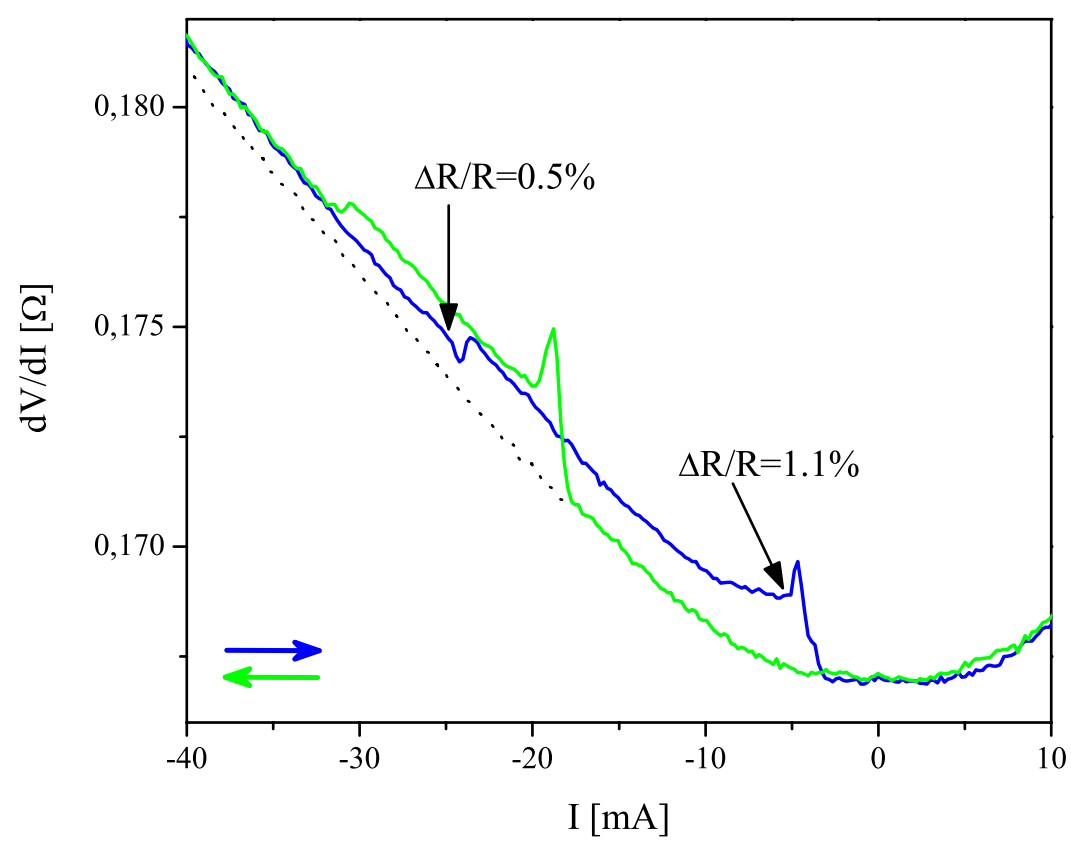

Figure 5.5: Current sweeps for a 3nmCo/10nmCu/12nmCo trilayer nanopillar (device $B$ ) in the field perpendicular to the plane geometry at $B=5 T$ and $T=10 K$.

Device B (see figure 5.5) exhibits a much smaller resistance change than device A: only $1.1 \%$ at $I=-5 \mathrm{~mA}$ directly after the characteristic peak structure, and this value is even reduced again at $I=-23.8 \mathrm{~mA}$ to $\Delta R / R=$ $0.5 \%$. The overall reduced GMR effect can be attributed to a possibly increased interface roughness of device $\mathrm{B}$. The magnetic moments of the atoms at intermixed regions are expected to be reduced or even misaligned with the magnetization of the Co layers [60], which would lead to a reduction in spin asymmetry and hence a suppression of GMR. The origin of the downward step at $I=-23.8 \mathrm{~mA}$ and the preceding plateau in $d V / d I$ is ambiguous: it might either indicate a transition from precessional states to a static antiparallel alignment (similar to the small plateau observed for device A) or a transition from a static state to another static state with a magnetization direction, which deviates stronger from a perfectly antiparallel alignment. The precise dependence of the magnetoresistance effect on the angle $\Theta$ between the two Co layers has been calculated by Slonczewski [40], and the result is depicted in figure 5.6. The magnetoresistance decreases with decreasing angle $\Theta$, and depending on the parameter $\chi$ this decrease deviates 
from linearity. $\chi$ is defined by the following relation:

$$
\chi=\frac{1}{2} A G\left(R_{+}+R_{-}\right)-1
$$

where $A$ denotes the area, $G$ is a constant depending on the spacer material, and $R_{+}$and $R_{-}$represent the corresponding channel resistances. So far only positive values of $\chi$ have been experimentally confirmed [20,26], and thus a curve similar to the one denoted with $\chi=2$ seems to be representative.

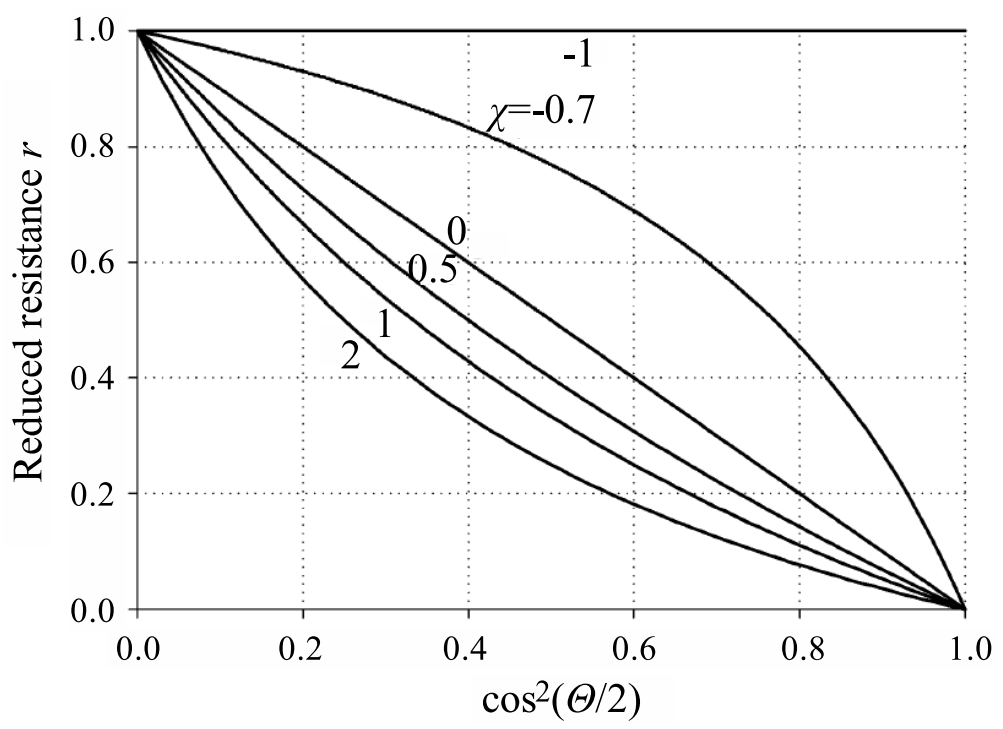

Figure 5.6: Reduced resistance $r=(R(\Theta)-R(0)) /(R(\pi)-R(0))$ as a function of the variable $\cos ^{2}(\Theta / 2)$. Taken from [40].

\subsubsection{Hysteretic Effects}

The current driven excitations occur at different critical currents for opposite DC current sweep directions. This hysteretic behavior can be understood by taking into account that the magnitude of the spin torque effect strongly depends on the angle $\Theta$ between the magnetization of the free and the fixed ferromagnetic layer. Here a corresponding theoretical model developed by Slonczewski [40] is briefly described.

Within this approach the reduced spin transfer torque $\tau_{\text {red }}$, normalized to the charge current $I$, is calculated as a function of $\Theta$ for a symmetric trilayer device with one pinned ferromagnetic layer. The resulting curves are displayed in figure 5.7. No spin torque arises for a collinear alignment of both magnetization directions, i.e. $\tau_{\text {red }}(0)=\tau_{\text {red }}(\pi)=0$. In between these two minima the magnitude of the spin torque effect depends on the parameter $\Lambda$. This parameter is connected to the deviation of the angular dependence of the GMR resistance from linearity parameterized by $\chi$ :

$$
\Lambda^{2}=\chi+1
$$




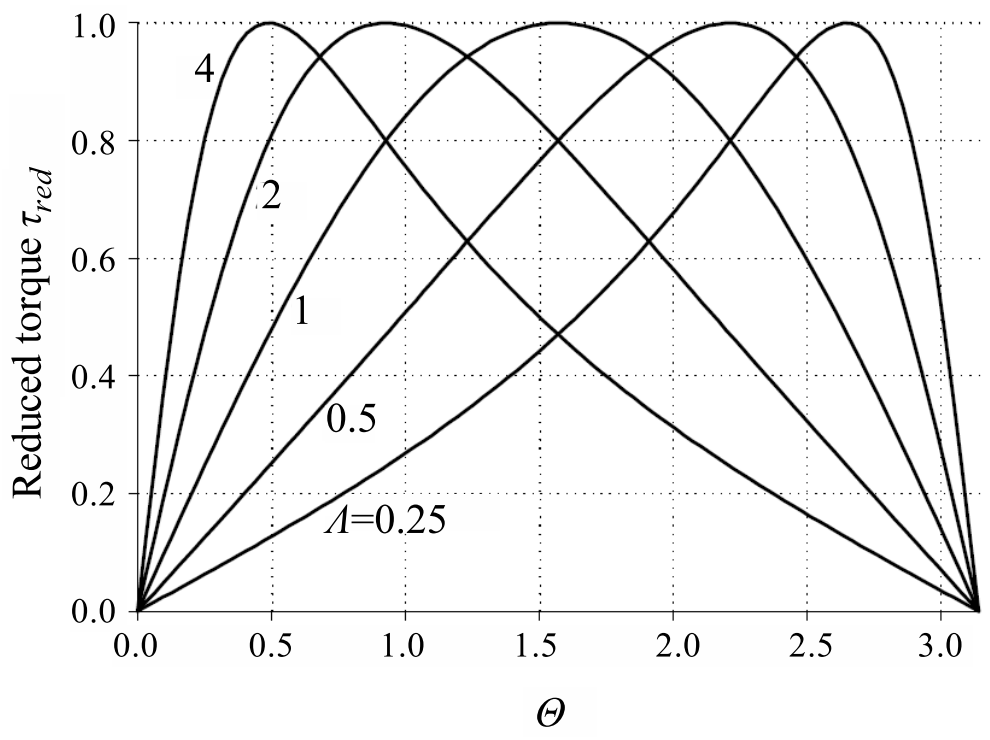

Figure 5.7: Calculated current-induced reduced torque $\tau$ as a function of the angle $\Theta . \Theta=0$ corresponds to a parallel alignment of the ferromagnetic layers, whereas $\Theta=\pi$ denotes the antiparallel configuration. The meaning of the parameter $\Lambda$ is explained in the text. Taken from [40].

The determination of the parameter $\chi$ has been discussed in the previous section. Positive values of $\chi$ in combination with 5.2 yield values $>1$ for $\Lambda$. Starting from parallel alignment $\Theta=0$ the spin torque thus increases only gradually, until a maximum value is reached between $\pi / 2<\Theta<\pi$. For angles larger than this maximum the spin torque falls rapidly until it vanishes completely at $\Theta=\pi$. This strong asymmetry of the angular dependence of the spin torque can be discovered in figures 5.3 and 5.5 as well: due to the theoretical findings the current driven torque effects are expected to increase faster for an initial deviation from the antiparallel configuration of the ferromagnetic layers than from the parallel state. This is indicated by the lower critical currents (absolute value of $I_{C}$ ) observed in the experimental data for the sweeps towards positive current bias.

\subsubsection{Field Dependence of the Observed Effects}

In order to get a general idea about the DC current dependence of the current-induced effects, the results of the current sweeps at fixed fields applied perpendicular to the plane are summarized in color plots. For these diagrams the background resistance (the $d V / d I(I)$-curve at zero field) is subtracted from each measurement, so that $\Delta R=R_{H \neq 0}-R_{H=0}$ can be plotted as a function of $I$ and $H$. The results are depicted in figure 5.8 for device $\mathrm{A}$ and in figure 5.9 for device $\mathrm{B}$. These color plots represent phase diagrams, in which the critical current $I_{C}$ separates the low resistance state, in which both Co layers are in or close to a parallel orientation, from a 
state of higher resistance, in which the relative orientation of the two layers clearly deviates from parallel alignment.
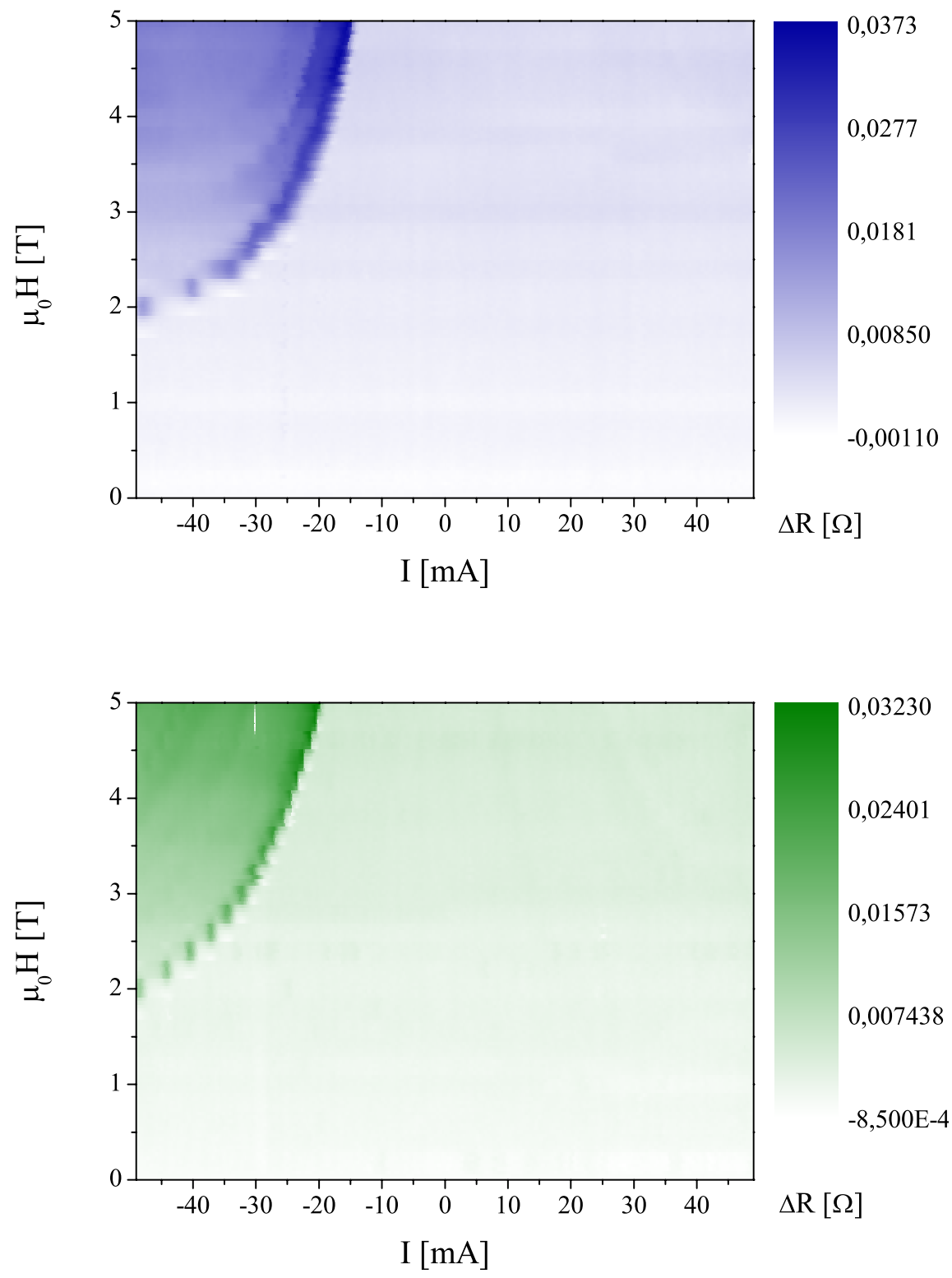

Figure 5.8: Color plots deduced from the current sweeps for the $3 \mathrm{nmCo} / 10 \mathrm{nmCu} / 12 \mathrm{nmCo}$ trilayer nanopillar (device A) in the field perpendicular to the plane geometry at $T=10 \mathrm{~K}$. The blue diagram represents the sweep direction from negative to positive current bias, whereas the green diagram corresponds to the opposite sweep direction. 

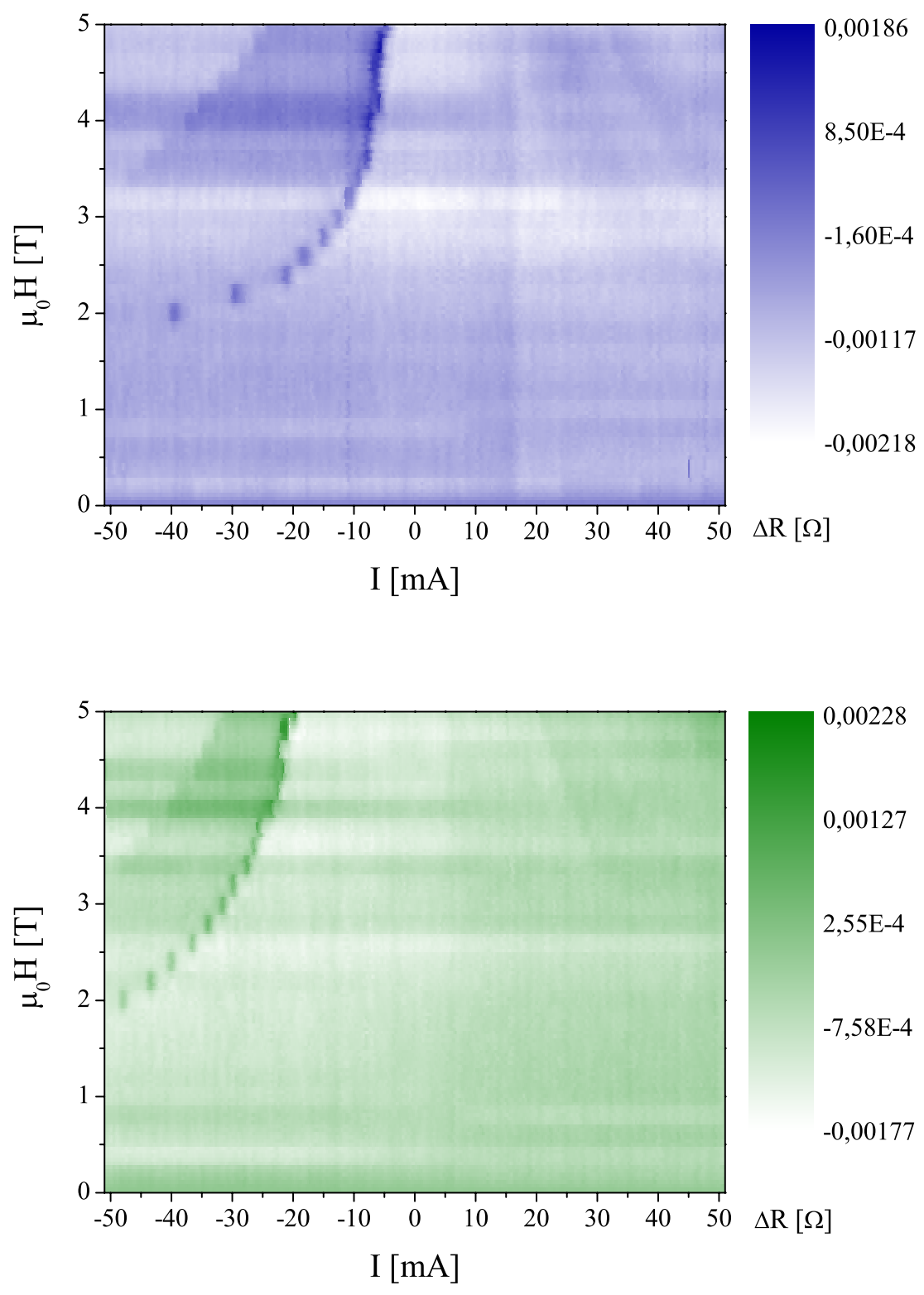

Figure 5.9: Color plots deduced from the current sweeps for the $3 \mathrm{nmCo} / 10 \mathrm{nmCu} / 12 \mathrm{nmCo}$ trilayer nanopillar (device B) in the field perpendicular to the plane geometry at $T=10 \mathrm{~K}$. The blue diagram represents the sweep direction from negative to positive current bias, whereas the green diagram corresponds to the opposite sweep direction.

For both devices and both sweep directions the critical current (absolute value) is found to decrease non-linearly with increasing field. This is to- 
tally contrary to the behavior expected from Slonczewski's critical current dependence as discussed in section 2.7.4, which predicts a linear increase of $I_{C}$ with increasing magnetic fields due to the dominating field term in equations 2.29 and 2.30. This increase of $I_{C}$ with higher external fields is also confirmed by many experimental studies in the high field regime $[66,67,70]$. However, the anomalous behavior of $I_{C}$ for devices A and B is rather similar to the results of the low field studies $\left(H<M_{S}\right)$ presented in $[20,70]$. In this field region the anomalous field dependence is attributed to the deviations of the magnetization direction from the perpendicular to the plane direction: this geometry might lead to a non-monotonic decrease of the FMR frequency with increasing field [20]. Since the FMR frequency in the high-field regime is directly proportional to the effective field and hence directly proportional to the critical current, it is quite plausible that also in the low field regime the critical current follows the field dependence of the FMR frequency. With respect to the results presented here in figures 5.8 and 5.9, this interpretation means that even in very high external fields the magnetization direction deviates from a perfect alignment with the external out-of-plane field.
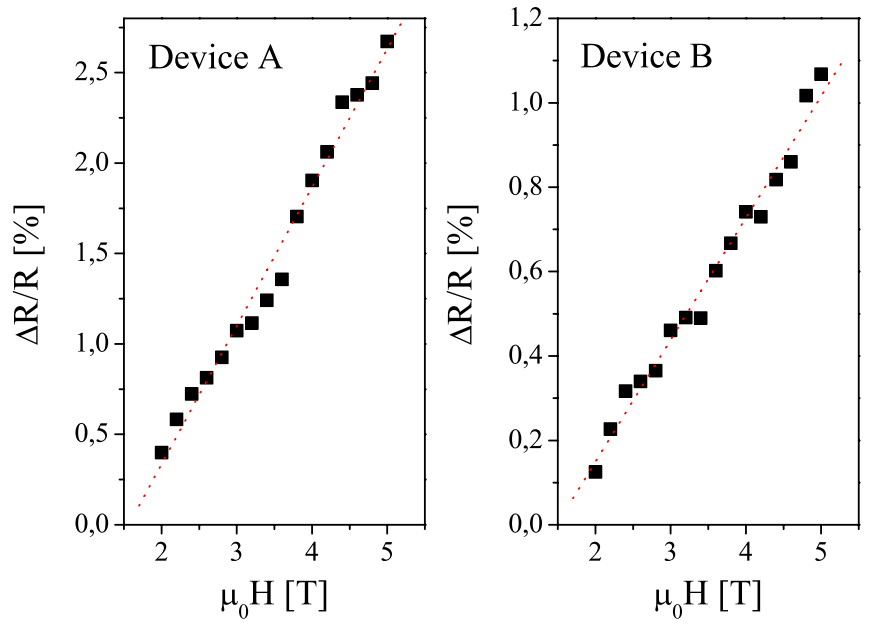

Figure 5.10: $\Delta R / R$ as a function of the external field for device $A$ and device $B$. The dotted red line is just a guide to the eyes.

This assumption is supported by the observed field dependence of the GMR effect. According to figure 5.10 the GMR ratio increases almost linearly with the external field, and there is no indication for a saturation of this effect even in very high field ranges. In conjunction with the relation for the magnetoresistance effect as a function of the angle $\Theta$ between the magnetization directions for both layers (see section 5.1.2, figure 5.6) this result can be interpreted in the following way: with increasing external fields the magnetization direction of the thin Co layer seems to turn more and 
more antiparallel to the direction of the external field, so that the angle $\Theta$ increases, which would lead to the detected increase in GMR ratio. However, the reasons for these peculiar magnetization configurations are not understood in detail.

\subsection{Field Sweeps at Fixed Current Bias in the Field Perpendicular Geometry}

In order to check the correlation between current-induced magnetization reversal (as discussed in the previous sections) and field-induced magnetization reversal, magnetoresistance measurements at fixed current bias were performed on device B. The corresponding results for field sweeps from $+5 \mathrm{~T}$ to $-5 \mathrm{~T}$ (green lines) and back to $+5 \mathrm{~T}$ (blue lines) for a current bias of $0 \mathrm{~mA}$, $-25 \mathrm{~mA}$ and $+25 \mathrm{~mA}$ are displayed in figure 5.11. The latter curve is shifted vertically by $-2.5 \mathrm{~m} \Omega$ for reasons of clarity.

The magnetoresistance loop for $0 \mathrm{~mA}$ bias current shows no significant abrupt changes in $d V / d I$, but it decreases gradually with increasing external field. This concerns both field directions. Furthermore, the curves for both sweep directions are nearly identical.

The measurements performed at $-25 \mathrm{~mA}$ yield significantly different results. First of all, the overall resistance of this curve is about $8 \mathrm{~m} \Omega$ higher than the resistance values at $0 \mathrm{~mA}$, because here Joule heating processes are relevant. For the further description of the curve four distinct field regions can be distinguished: for negative magnetic fields in the regime $H^{\prime}$ for both sweep directions no sharp changes in $d V / d I$ are detected and the curvature of this measurement is identical to the one recorded at $0 \mathrm{~mA}$. The same applies for the low field positive regime $H_{1}$ for both sweep directions. The high field region of $\mathrm{H}_{2}$ is marked by a peak structure for the sweeping direction from positive current bias to negative current bias (green), which is followed by a plateau of higher resistance. For the opposite sweep direction (blue) no anomalies within $H_{2}$ are visible, but in the very high field regime of $H_{3}$ a characteristic peak structure is observed. This feature is followed by a high resistance state, which is equal in magnitude to the plateau for the opposite sweep direction.

The third magnetoresistance curve recorded at $+25 \mathrm{~mA}$ current bias is the 'mirror image' of the measurement at $-25 \mathrm{~mA}$ : here the features, which are observed within the high field regions $\mathrm{H}_{2}$ and $\mathrm{H}_{3}$ for negative current bias, are found for the inverse field direction within the regions $H_{2}^{\prime}$ and

$H_{3}^{\prime}$. The effects are of equal magnitude and occur at comparable critical fields: $3.8 \mathrm{~T}$ and $-3.8 \mathrm{~T}$ for the transition from the low resistance state to the high resistance state, and $1.7 \mathrm{~T}$ and $-1.9 \mathrm{~T}$ for the transition from the high resistance state to the low resistance state, respectively. Only the behavior observed at positive fields for the measurement at $+25 \mathrm{~mA}$ deviates a little bit from the curve shape at opposite fields at $-25 \mathrm{~mA}$ current bias; i.e. the curvature for the sweep from negative to positive fields is higher than for $0 \mathrm{~mA}$ bias current, and even some excitations in the form of small dips can 
be seen at $\mu_{0} H=3.4 \mathrm{~T}$ and $\mu_{0} H=3.8 \mathrm{~T}$.

An interpretation of these results in terms of a comparison to the current-induced spin transfer-torque effects can be found in the following section 5.2.1.

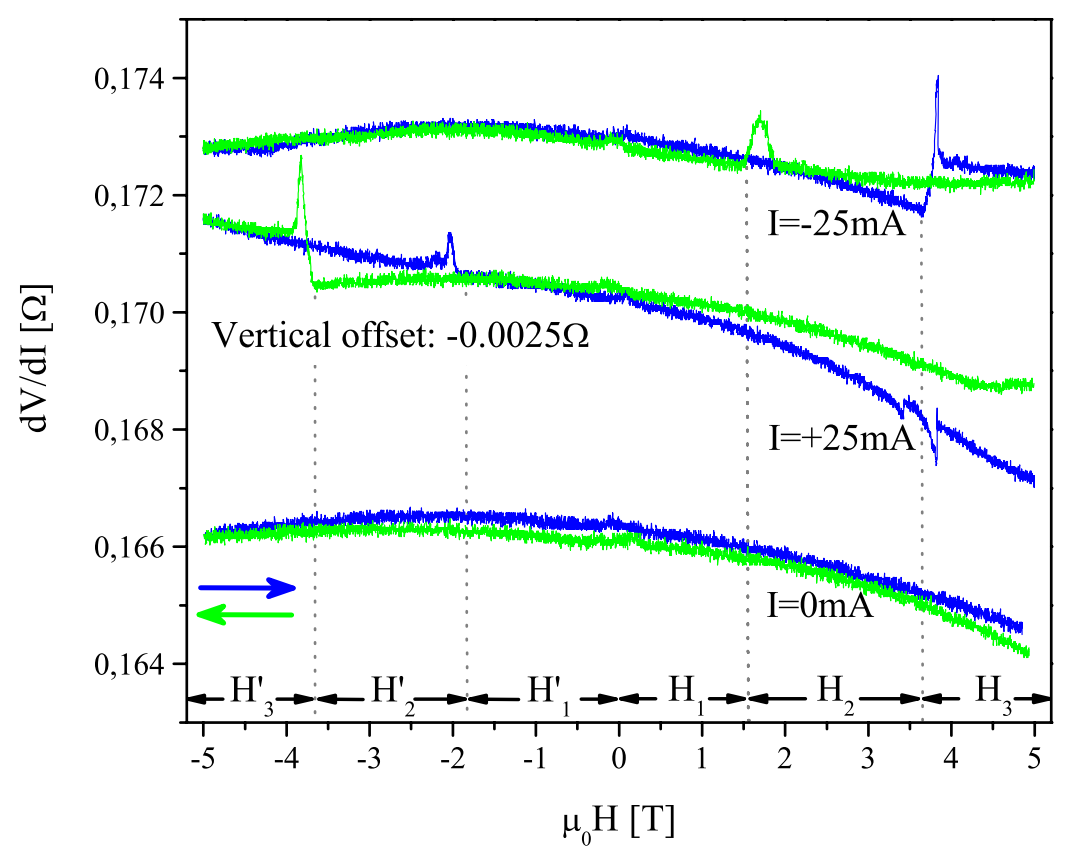

Figure 5.11: Field sweeps at $T=10 \mathrm{~K}$ for the $3 \mathrm{nmCo} / 10 \mathrm{nmCu} / 12 \mathrm{nmCo}$ trilayer nanopillar (device B) in the field perpendicular to the plane geometry with a constant current bias of $0 \mathrm{~mA},-25 \mathrm{~mA}$ and $+25 \mathrm{~mA}$, respectively. Blue lines indicate a sweep direction from $-5 T$ to $+5 T$, whereas the green curves refer to the opposite sweep direction.

\subsubsection{Field-Induced Effects versus Current-Induced Effects in the Field Perpendicular Geometry}

The absence of any excitations at $0 \mathrm{~mA}$ bias current is already expected from the current sweeps, where independent of the external field strength also no resistance changes are detected, which is contrary to the results reported in [20]. The reason for this can be attributed to the special magnetic configuration within the $12 \mathrm{~nm}$ polarizing Co layer: as already mentioned in section 5.1.2, the energetically lowest configuration is the magnetic vortex state. With increasing perpendicular magnetic field, the magnetization of the vortex then turns gradually out of plane. Obviously the same gradual transition takes place in the $3 \mathrm{~nm}$ Co layer, i.e. here the homogeneous in- 
plane magnetization also tilts slowly towards the increasing magnetic field. In this way the negative curvature of the magnetoresistance measurement can be explained: for increasing fields the magnetizations of the two Co layers are aligned more and more parallel to the direction of $H$, which results in a gradual decrease of the resistance of device B. The fact that there exists evidently no sharp jump from an in-plane to an out-of-plane magnetization for the 3nm Co layer above its demagnetization field but rather a gradual transition, might be ascribed to the interaction of the dipole fields of both Co layers.

The field sweep at a current bias of $-25 \mathrm{~mA}$ exactly reflects the results obtained for the current sweeps. The hysteretic transition from a parallel to an antiparallel alignment once more underlines, that the high resistance state is most likely due to a reorientation which results in a static configuration. In fact, even the locations of the peak structures, which are associated with spin-wave modes indicating the starting point of the switching process, coincide. Since the total effect of $\Delta R / R$ increases with increasing external field in the region of $H_{2}$ and $H_{3}$, respectively, the configuration of the two magnetization directions indeed becomes more and more antiparallel. The only feature in the field sweeps, which cannot be identified in the current sweeps, are the dips in $d V / d I$ for positive current bias $+25 \mathrm{~mA}$ and positive field values. These dips might be related to excitations in the thick $12 \mathrm{~nm}$ Co, which are discussed more explicitly in section 5.3.2.

However, from the spin torque model introduced in section 2.7.2, the field sweeps are expected to be symmetric around zero; i.e. the effects detected at positive field values should also appear for opposite external fields (independent of the polarity of the current). This has also been observed in similar experiments performed by Özyilmaz et al. [20, 66]. The absence of this symmetry for the data presented here might indicate a more complex configuration than a simple single domain state within the Co layers even in extremely high fields. Thus the limitations of the macrospin model are revealed. However, the origins of the possibly not fully homogeneous magnetization within the Co layers cannot be identified.

Overall the phase boundaries between the high and the low resistance state deduced from the current sweeps show an excellent agreement with the results of the field sweeps. This also applies for the hysteresis observed at the phase boundaries and the presence of additional dynamic excitations indicating the beginning of the reversal process. This correspondence suggests that the current-induced resistance change in high external fields is identical to the field-induced resistance change at high current bias. Last but not least the results for the field sweeps at fixed current bias avoid the complications caused by the varying background resistance as discussed in section 5.1.1. Hence, these results are a convincing argument against an explanation of the observed effects simply in terms of heating processes. 


\subsection{Current Sweeps at Fixed Applied Fields in the Field Parallel Geometry}

In order to obtain some more information on the nature of the currentinduced excitations and their dependence on the external field, additional experiments in the field parallel to the sample plane geometry have been performed. Figure 5.12 shows the corresponding current sweeps for device A.

At first sight these results do not deviate significantly from the results in the field perpendicular to the plane geometry displayed in figure 5.1; i.e. these curves also show a peak structure above the threshold current $I_{T}$, followed by a small high resistance plateau and the high resistance state for $I>I_{C}$. Also the field dependence of the characteristic features looks very similar to the one discussed in section 5.1.4. However, the overall magnetoresistance effect is slightly reduced. The same applies to the threshold current $I_{T}$ and the critical current $I_{C}$. Besides, for high positive current bias the curvature of the $d V / d I$ measurements gradually increases and then again decreases in high external fields.

The current sweeps in the field parallel geometry for device B (figure 5.13) show a totally different behavior. Here the high resistance state is found in a certain current region around zero DC current bias in the high field region above $1.8 \mathrm{~T}$. Again, a characteristic peak structure can be observed for negative current bias for both sweep directions. For even higher magnetic fields $\mu_{0} H>3.2 \mathrm{~T}$ a gradual decrease in $d V / d I$, which eventually evolves to a downward hysteretic step, is visible for sufficient negative current bias. However, the most intriguing features can be seen for positive current polarity: here a hysteretic downward step in $d V / d I$ can be noticed with the decrease emerging at lower current values for increasing current (blue line). For external field values with $1.8 \mathrm{~T}<\mu_{0} H<2.4 \mathrm{~T}$ the direction of the hysteresis is reversed, i.e. the decrease occurs at higher current values for increasing current (blue curve). Moreover, for $\mu_{0} H \geq 4.8 \mathrm{~T}$ a small dip in the differential resistance at $I=+41 \mathrm{~mA}$ followed by a peak is detected for current sweeps from positive to negative bias (green line). The characteristics described above all show a very similar field dependence: just as in the field perpendicular geometry the threshold or rather the critical currents decrease with increasing fields.

\subsubsection{High Resistance State at Zero Current Bias: Dipolar Coupling versus Switching to Vortex States}

The high resistance state observed in magnetic fields larger than $1.8 \mathrm{~T}$ at zero DC current bias for device B (see figure 5.14) can be explained by two different approaches: either it is attributed to the dipolar coupling mechanism between the two Co layers, or it is ascribed to a transition from a parallel single domain state (high resistance) to a vortex state (lower resistance) in the thin Co layer. Both approaches are briefly discussed in this section. 


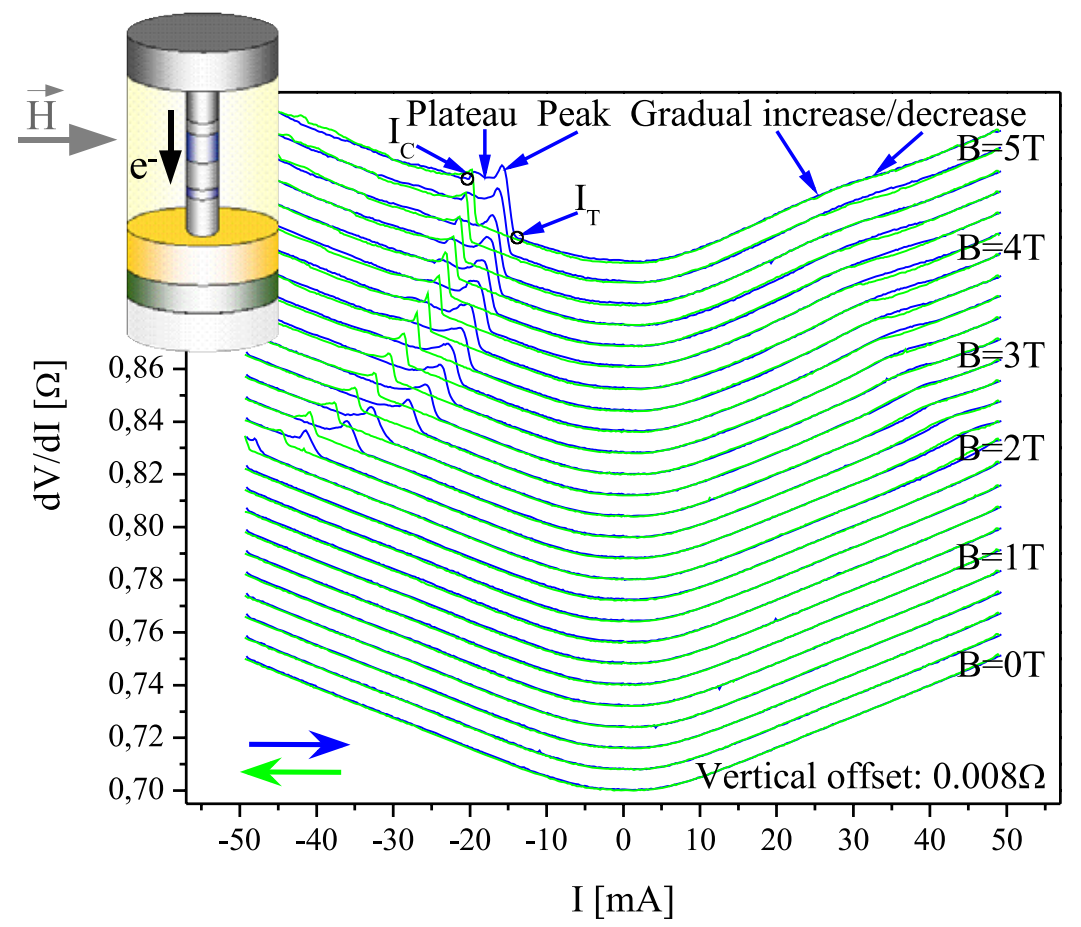

Figure 5.12: Current sweeps at $T=10 \mathrm{~K}$ for the $3 \mathrm{nmCo} / 10 \mathrm{nmCu} /-$ $12 n m C o$ trilayer nanopillar (device A) in the field parallel to the plane geometry. The curves for different magnetic field values are shifted vertically by $8 \mathrm{~m} \Omega$. The inset displays the direction of positive current flow and the corresponding magnetic field direction.

The coupling effect due to the dipolar long range interaction (also referred to as stray field effect) between the two Co layers might play a crucial role when studying their possible magnetic states and relative orientations. In zero magnetic field, the $12 \mathrm{~nm}$ thick Co layer is expected to remain in the vortex state, i.e. its strayfield is minimized. However, in sufficiently high external fields applied parallel to the plane, the magnetic configuration within this layer will gradually pass into a homogeneously magnetized in-plane state. Thus a considerable magnetic dipole field will emerge. Since the $3 \mathrm{~nm}$ thin Co layer is magnetized in-plane as well, the effect of dipolar coupling has to be taken into account for sufficient external fields. Figure 5.15 shows that for in-plane fields magnetic poles at the edges of the two Co layers then couple antiferromagnetically in order to minimize the magnetic energy. This might explain the observed high resistance state for zero bias current in high external fields for device B. However, the antiparallel dipolar coupling is expected to vanish in very high magnetic fields, where the layers should be stabilized in a parallel configuration due to the prevailing 


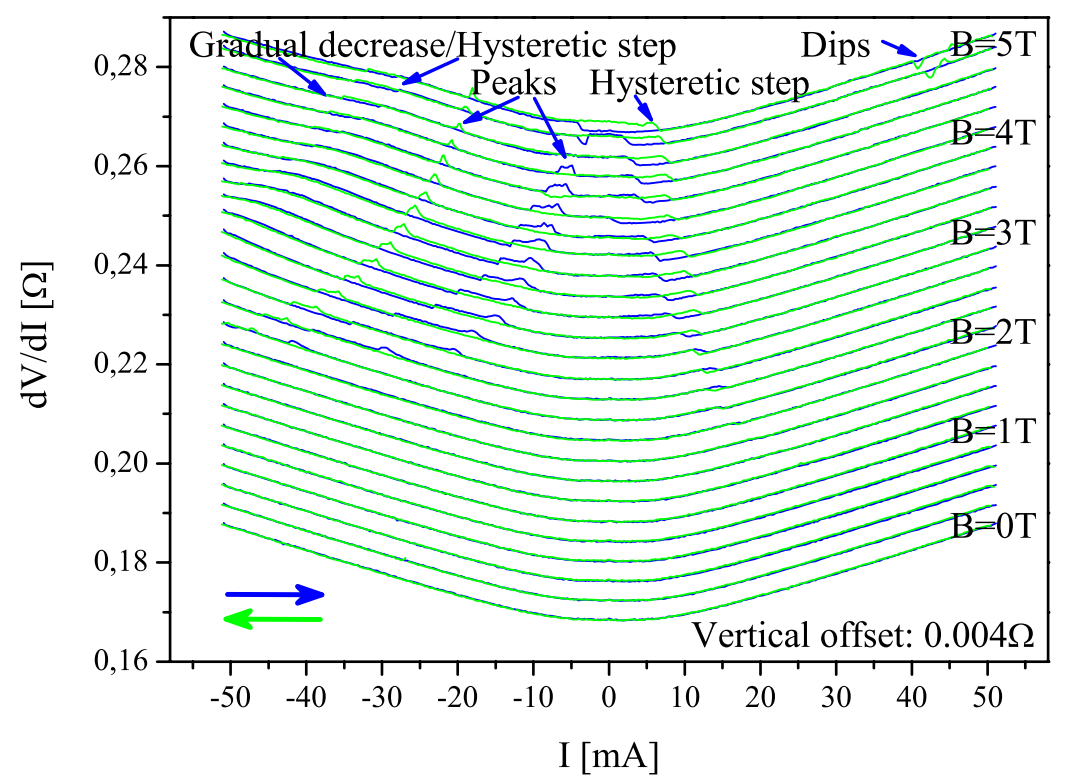

Figure 5.13: Current sweeps at $T=10 K$ for the $3 \mathrm{nmCo} / 10 \mathrm{nmCu} / 12 \mathrm{nmCo}$ trilayer nanopillar (device $B$ ) in the field parallel to the plane geometry. The curves for different magnetic field values are shifted vertically by $4 \mathrm{~m} \Omega$.

Zeeman energy. But a transition back to the parallel configuration for even higher fields is not found in the data for device B. Hence, an interpretation of the high resistance state in terms of dipolar coupling seems to be rather inadequate.

An alternative approach is the consideration of current-induced vortex states. Here both Co layers of device B are expected to be aligned with the external field for field values above the demagnetization field at zero current bias, and the effect of dipolar coupling is assumed to play only a minor role. This means that the two layers show a homogeneous in-plane magnetization. For higher DC current bias, the Maxwell-Oersted field of this current might be sufficient to facilitate the nucleation of a vortex state (either in only one Co layer or even in both layers). Figure 5.16 demonstrates, how the existence of a vortex might lead to a decrease in the differential resistance as it is detected for high bias currents (or for zero DC bias current in low external magnetic fields, respectively). Urazhdin et. al suggest that the reduced resistance occurs due to a suppression of spin accumulation in the vortex state [73]. Figure 5.16 (a) shows that the polarized electron (solid arrow) becomes non-collinear to the local magnetization upon diffusing angularly by $\varphi$ (dashed arrow). Since the electron then possesses spin components 


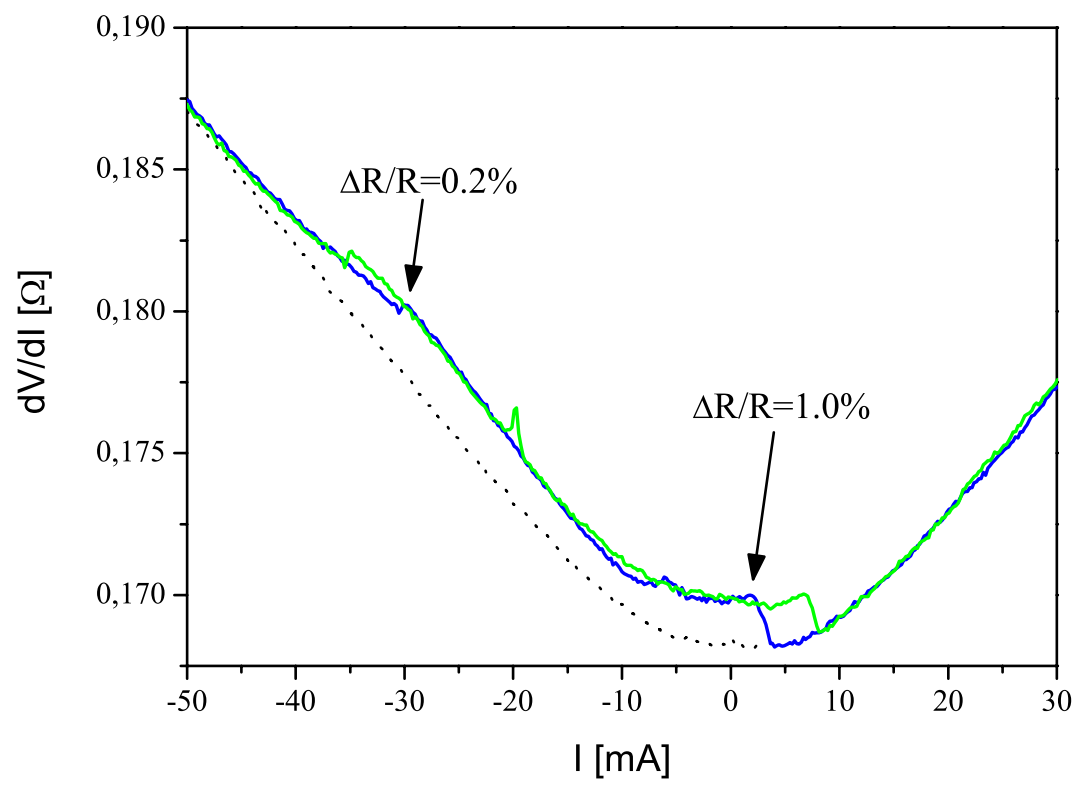

Figure 5.14: Current sweeps for a 3nmCo/10nmCu/12nmCo trilayer nanopillar (device B) in the field parallel to the plane geometry at $B=4.6 T$ and $T=10 K$.
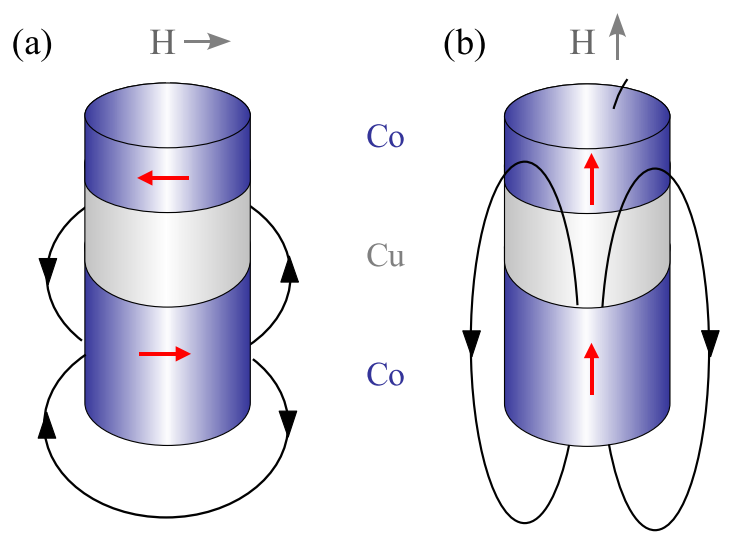

Figure 5.15: Dipolar coupling resulting in (a) an antiparallel layer configuration for in-plane fields and (b) a parallel layer configuration for out-ofplane fields.

both parallel and antiparallel to the local magnetization, it effectively relaxes its polarization by diffusion. This leads to a suppression of the spin accumulation in the vortex state, i.e. to a decrease in $d V / d I$. Urazhdin et al. call the effect described above topological spin relaxation, because it is a 
(a)

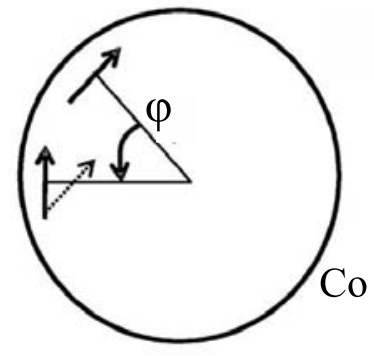

(b)

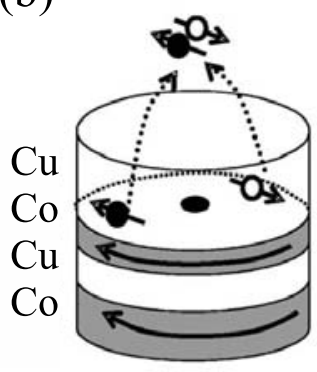

Figure 5.16: Spin relaxation effects in the vortex state due to (a) angular diffusion (b) spin diffusion towards the center of the vortex. Adapted from [73].

direct consequence of the topology of the vortex state, which is independent of the spin-flipping rate of the corresponding material [73]. Figure 5.16 (b) displays another possible spin relaxation process, which is also related to the topological spin relaxation effect. By diffusing radially from opposite sides of the circular Co layer, the spin polarizations of two electrons cancel each other out at the center of the layer, hence creating an extra channel of effective spin relaxation [73].

Although the mechanisms described above seem to explain the observed high resistance state and the subsequent decrease in $d V / d I$ for increasing bias currents (see figure 5.14) very well, there are a few critical points concerning the application of these models to the data presented here. First of all it should be noted that the proposed models are essentially applied in the low field regime $B \leq 50 \mathrm{mT}$ in reference [73], and it is not at all obvious that for nanopillars with a diameter below 100nm the Maxwell-Oersted field of a current is sufficient to lead to the formation of a vortex state in the very high field regime. ${ }^{1}$ Secondly, it is not clear why vortices can nucleate in the field parallel to the sample plane geometry, while they do not seem to be present in the field perpendicular to the plane geometry (cf. section 5.1). Also the quite different switching mechanisms observed for device A do not agree with the models of Urazhdin et al.. However, the latter discrepancy can be ascribed to slight geometrical deviations between device A and B, which have already been indicated by their different total resistivities as well as the dissimilar critical or threshold current densities required for the observation of current-induced excitations.

\subsubsection{Static Excitations versus Dynamic Excitations}

In analogy to section 5.1.2 for the field perpendicular geometry, it is reasonable to attribute all hysteretic changes in the differential resistance to an eventually static change in the magnetization configuration for the field par-

\footnotetext{
${ }^{1}$ In fact, a rough estimation of the magnetic field with the Biot-Savart law for a current of $50 \mathrm{~mA}$ yields only $B=200 \mathrm{mT}$ at a radial distance of $50 \mathrm{~nm}$.
} 
allel to the plane experiments as well. Concerning device A this means that the increase in $d V / d I$ at $I_{T}$ denotes the onset of large angle excitations which lead to a transition of the magnetization in the $3 \mathrm{~nm}$ Co layer to a close to antiparallel static alignment for $I>I_{C}$ (cf. green curve in figure 5.17). The gradual resistance increase (figures 5.12 and 5.17) for the opposite current polarity on the other hand is completely reversible, wherefore it is ascribed to dynamic excitations. Since the underlying mechanism for excitations at positive bias might be fundamentally different to the one described so far (section 2.7.2), it is discussed further in the last section 5.3.3.

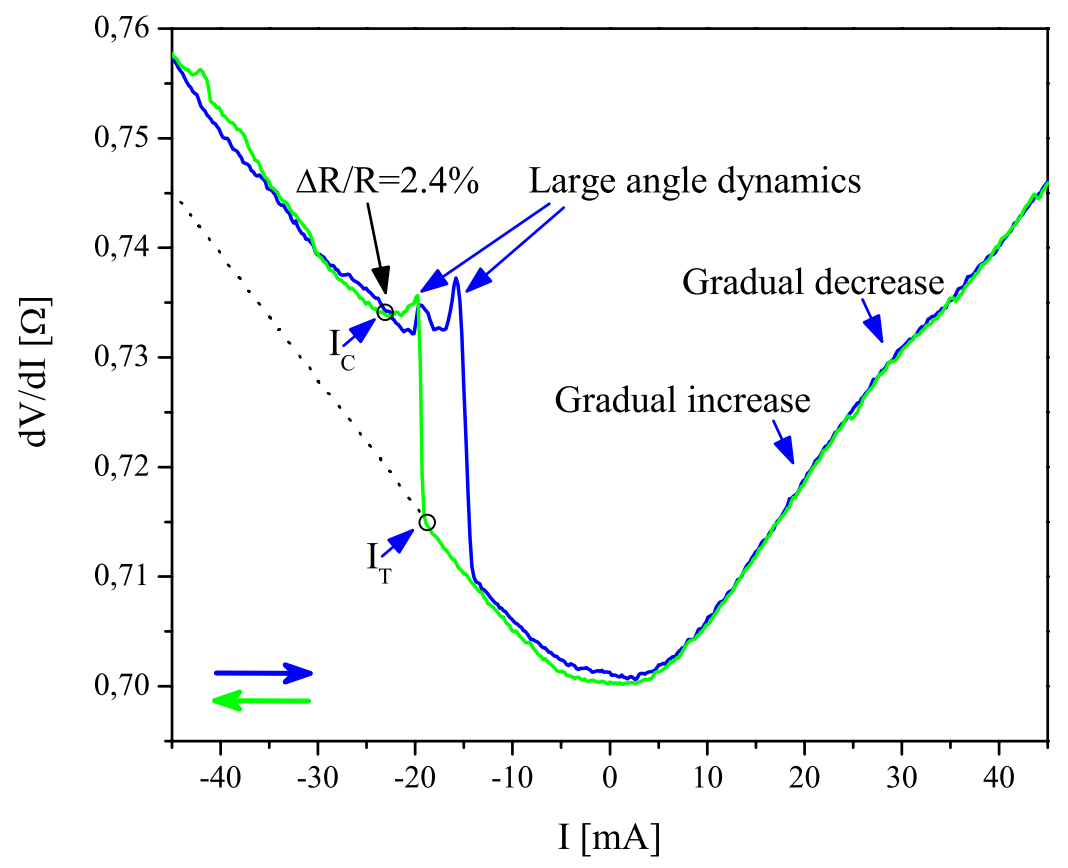

Figure 5.17: Current sweeps for a 3nmCo/10nmCu/12nmCo trilayer nanopillar (device $A$ ) in the field parallel to the plane geometry at $B=5 T$ and $T=10 K$.

Due to the different initial situation in device B (high resistance state at zero current bias), also the interpretation of the observed changes in $d V / d I$ is a little bit more tricky. According to what has been said in the previous section, the high resistance state at zero current bias is ascribed to a parallel alignment of the two Co layers in high external fields $\left(\mu_{0} H=4.6 \mathrm{~T}\right.$ in figure 5.18). The downward step at $-35.3 \mathrm{~mA}$ (sweep direction towards negative current bias; green curve) might indicate that a vortex core enters one of the Co layers, which instantaneously leads to a small decrease in resistance due to the mechanisms described in section 5.3.1. The subsequent gradual 


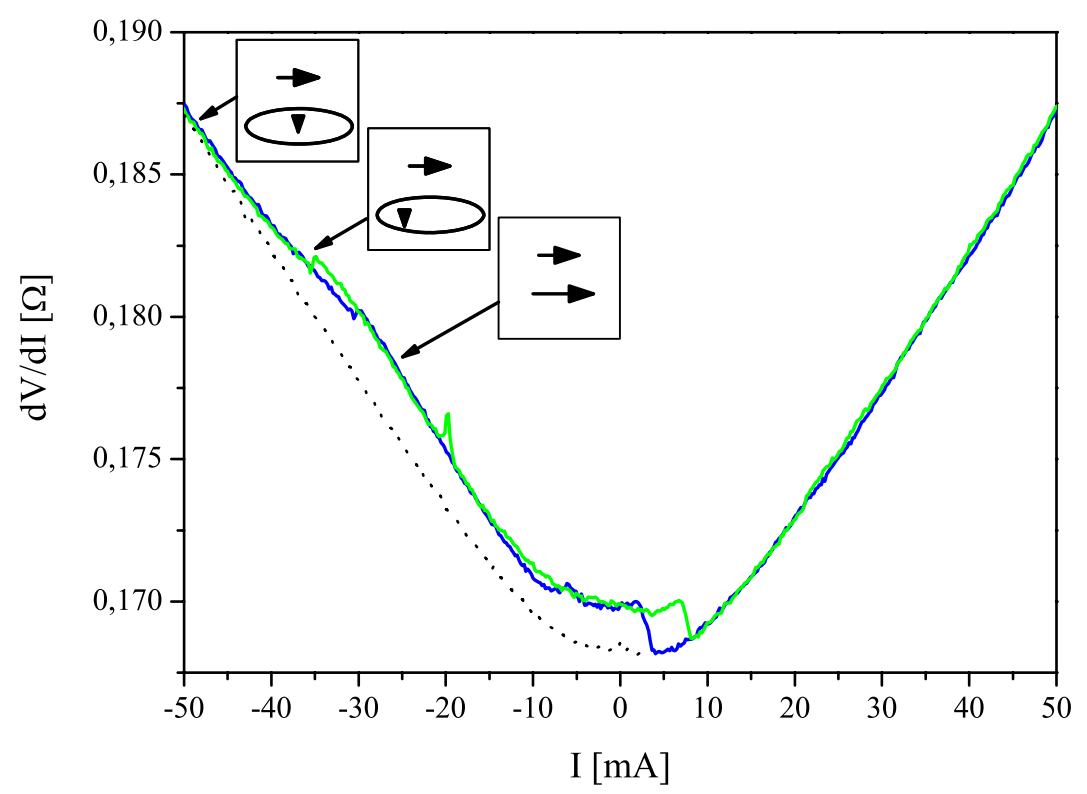

Figure 5.18: Current sweeps for a 3nmCo/10nmCu/12nmCo trilayer nanopillar (device B) in the field parallel to the plane geometry at B $=4.6 T$ and $T=10 K$. The insets schematically show the parallel configuration, the nucleation of the vortex core and the vortex core moving towards the center of the Co layer, respectively.

decrease in $d V / d I$ could result from the vortex core moving towards the center of the Co layer, because with increasing $I$ the effect of the OerstedMaxwell field more and more dominates over the influence of the effective magnetic field. In this way the Co becomes even more magnetically inhomogeneous on the nanoscale, which would definitely cause a further resistance suppression. The similar behavior for the reversed sweep direction (blue line) can be explained by the vortex core moving successively out of the Co disk due to the predominant importance of the effective magnetic field for lower bias currents. However, the peak structure recorded at $I=-19.7 \mathrm{~mA}$ most likely originates from precessional motion of the magnetization in the thin Co layer. The exact origin of these dynamic excitations and why they are observed for one current sweep direction only is not really clear. All changes detected in $d V / d I$ for positive current bias are discussed further in section 5.3.3.

\subsubsection{Excitations for Positive Current Bias}

The most prominent feature observed at positive current bias is the downward step in $d V / d I$ for fields above $1.6 \mathrm{~T}$ for device B (cf. figures 5.13 
and 5.14). Although this transition to the low resistance state looks a little bit different than the one at negative current bias, it can be attributed to a similar mechanism: due to the symmetry of the Maxwell-Oersted field the emergence of a vortex state in one of the Co layers is also expected for sufficient currents with positive polarity. Here only the helicity of the vortex is reversed, but the effect of the topological spin relaxation process described in figure 5.16 stays the same, i.e. the resistance is effectively reduced due to angular spin diffusion.

The occurrence of excitations at positive current bias is somewhat astonishing due to the model introduced in section 2.7.2. Within this approach positive currents are rather expected to repress any deviations of the 'free' layer magnetization away from parallel alignment with the 'fixed' layer.
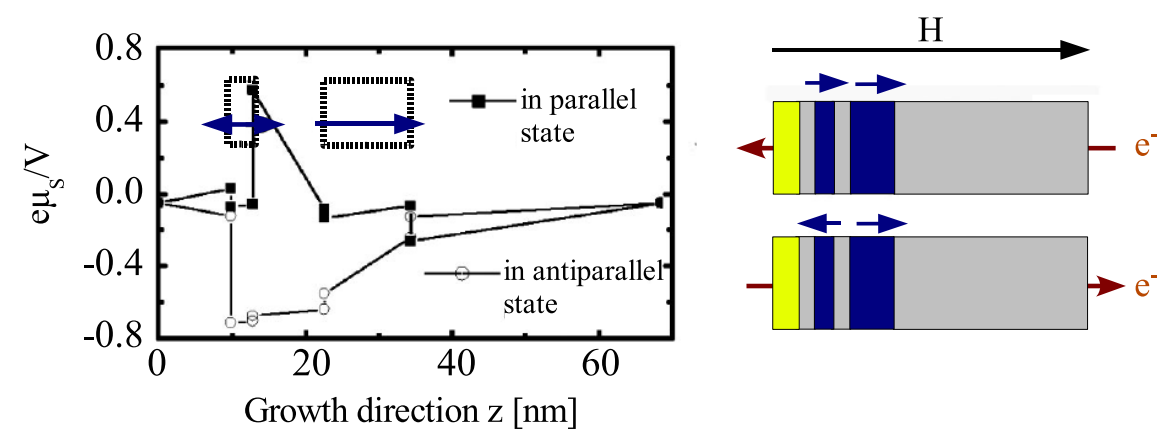

Figure 5.19: The calculated spin accumulation in the parallel state (top) for positive currents and in the antiparallel state for negative currents (bottom). Taken from [74].

Anyhow, in the field parallel to the plane geometry changes in $d V / d I$ at positive $I$ are evident - especially for device B. According to Özyilmaz et al. dips in the resistance can be interpreted in terms of spin wave instabilities, which are caused by strong asymmetries in the longitudinal spin accumulation [74]. They point out that dips in $d V / d I$ have also been detected in nanopillars with only a single ferromagnetic layer [9], where the necessary condition for such instabilities is the following: the current bias has to be such that the overall longitudinal spin accumulation on either side of the affected ferromagnetic layer is opposite to its magnetization direction. Özyilmaz et al. have calculated the corresponding spin accumulation pattern in the parallel and the antiparallel state using the two-current model [74]; their results are displayed in figure 5.19. Here the spin accumulation about the 'fixed' layer is clearly asymmetric in the parallel configuration at positive current bias $^{2}$, so that the net spin accumulation is opposite to the magnetization direction. Due to the theoretical models presented in $[42,43]$, these accumulation patterns can cause dynamically inhomogeneous states even in

\footnotetext{
${ }^{2}$ The sign convention for the current polarity in [74] is opposite to the sign convention used for the specific sample and magnetic field configuration presented here, i.e. now the term positive current bias refers to the negative current polarity in [74].
} 
the thicker 'fixed' layer. However, the initial magnetization configuration for device $\mathrm{B}$ and thus also the longitudinal spin accumulation pattern deviate significantly from the one for the samples in reference [74]. Hence, it is questionable if analogous conclusions can be drawn for the excitations shown in figure 5.20 and if the dips can be ascribed to the occurrence of spin waves within the thick Co layer.

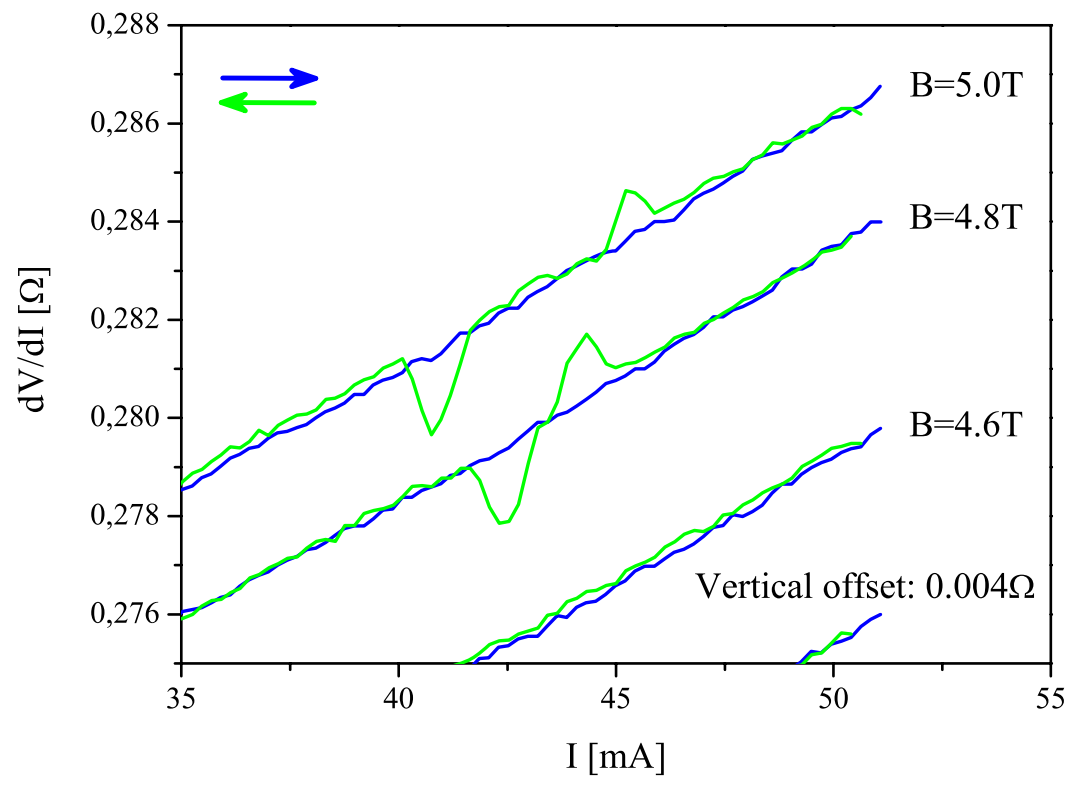

Figure 5.20: Dips (and peaks) observed in $d V / d I$ at positive current bias for $B=4.8 T$ and $B=5.0 T$ for the $3 \mathrm{nmCo} / 10 \mathrm{nmCu} / 12 \mathrm{nmCo}$ trilayer nanopillar (device B) in the field parallel to the plane geometry at $T=10 \mathrm{~K}$.

The arguments given by Özyilmaz et al. [74] seem to be more applicable for device A, because here no evidence for a vortex state has been found. Thus the magnetization directions of the two Co layers are assumed to be parallel in the low resistance state at positive current bias. However, for field values above $1.8 \mathrm{~T}$ and sufficiently high current densities a gradual increase (followed by a gradual decrease for even higher currents) in the differential resistance is detected (cf. figure 5.12). According to the arguments stated in the previous paragraph, this rise in $d V / d I$ can be attributed to currentinduced instabilities in the thick Co layer.

Some conclusions on the nature of the dynamic excitations can be drawn from analyzing the origins of dips and peaks: uniform excitations are expected to cause an increase in $d V / d I$, because in the parallel configuration they should only lead to an enhanced GMR. But then non-uniform excitations can account for the reduction in the differential resistance, since they 
effectively decrease the spin accumulation in the $\mathrm{Cu}$ spacer layer due to mixing the two spin channels [42]. Hence, the device resistance is reduced and dips in $d V / d I$ can be observed.
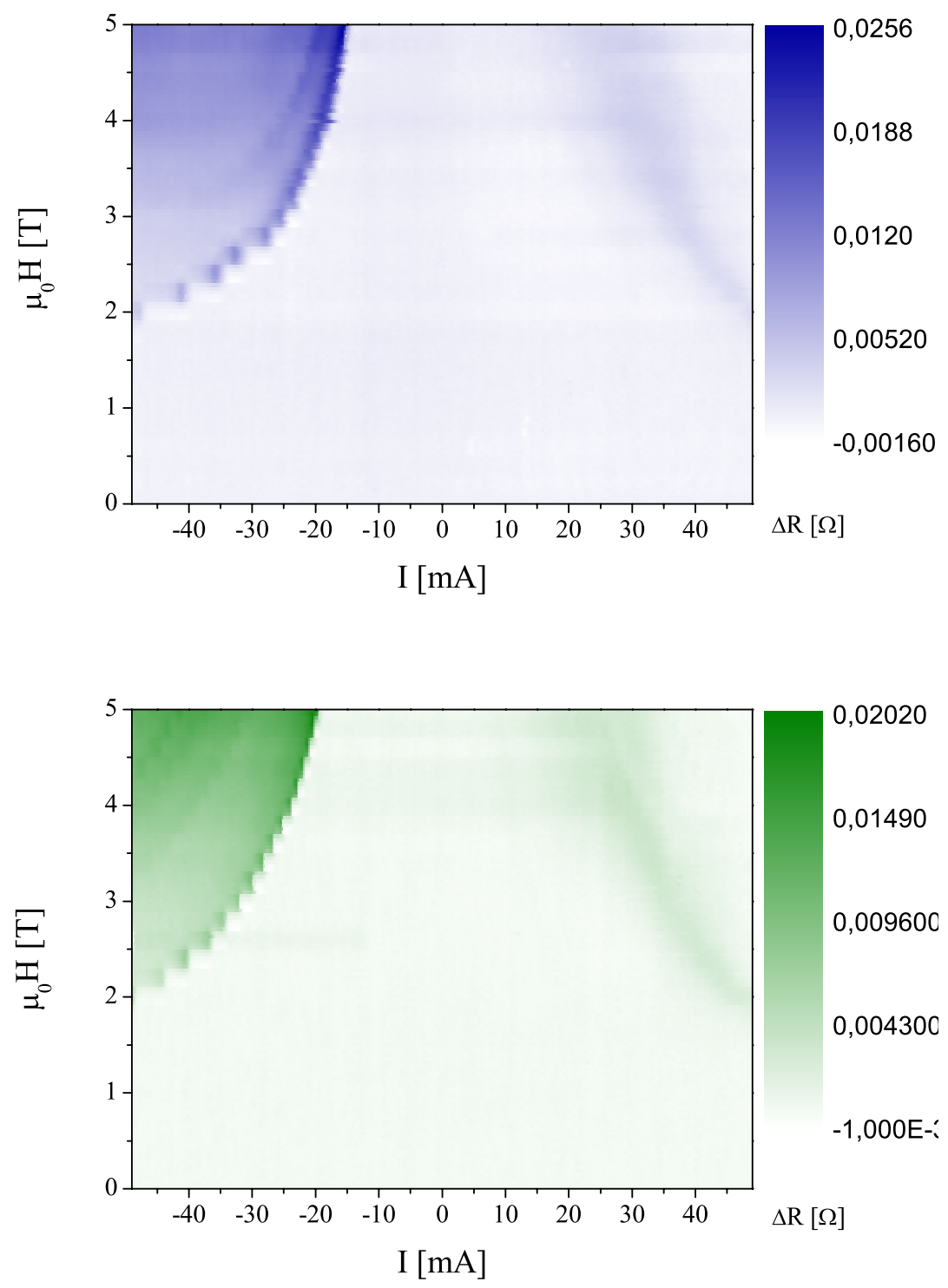

Figure 5.21: Color plots deduced from the current sweeps for the 3nmCo/$10 \mathrm{nmCu} / 12 \mathrm{nmCo}$ trilayer nanopillar (device A) in the field parallel to the plane geometry at $\mathrm{T}=10 \mathrm{~K}$. 


\subsubsection{Field Dependence of the Observed Effects}

The results of the current sweeps in the field parallel geometry are summarized in the color plots of figures 5.21 and 5.22 for device A and device B, respectively. Corresponding to the derivation of the plots presented in section 5.1.4, the $d V / d I(I)$-curve at zero field has been subtracted from each measurement, so that $\Delta R=R_{H \neq 0}-R_{H=0}$ for both devices is displayed in
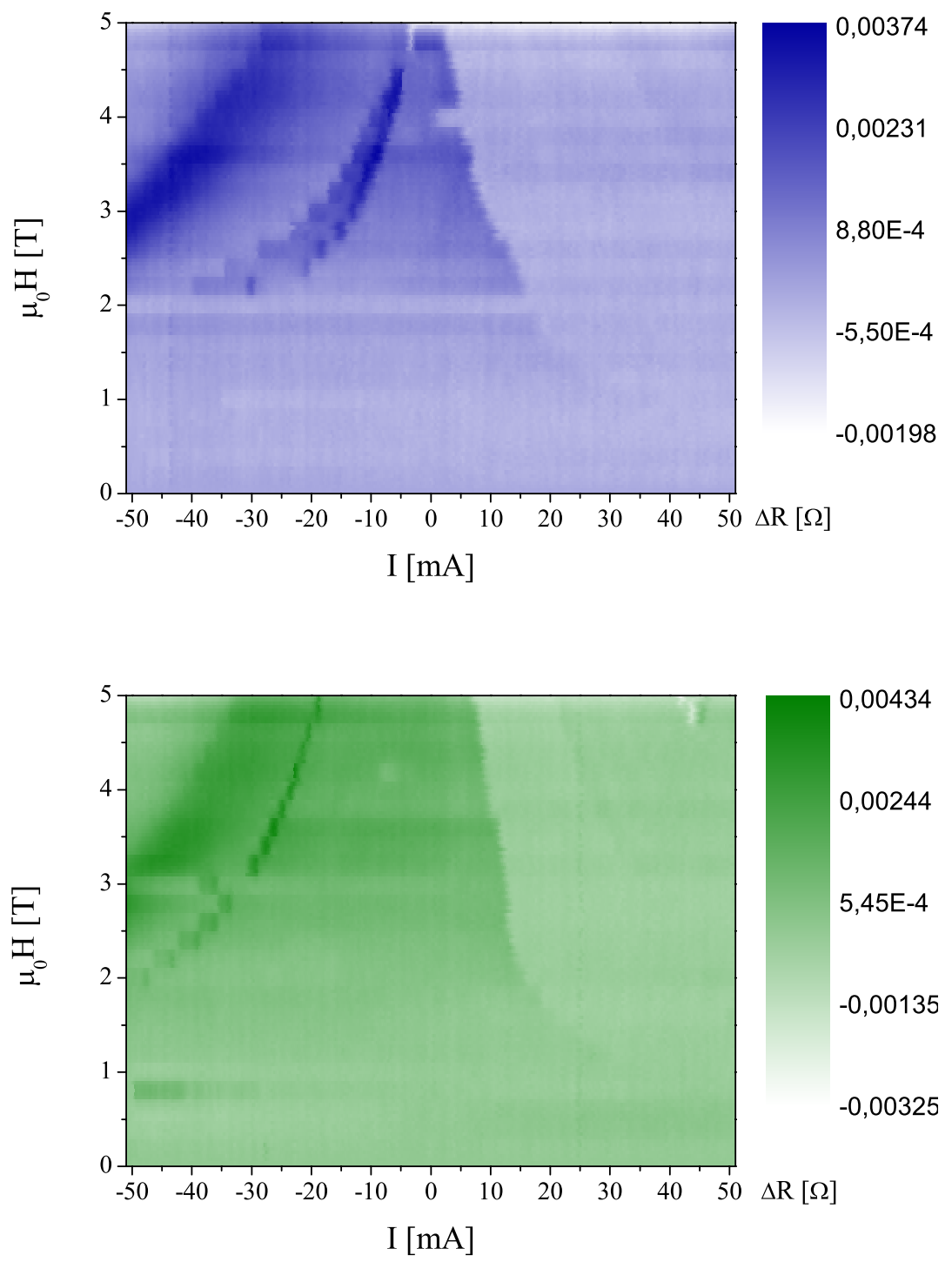

Figure 5.22: Color plots deduced from the current sweeps for the 3nmCo/$10 \mathrm{nmCu} / 12 \mathrm{nmCo}$ trilayer nanopillar (device B) in the field parallel to the plane geometry at $T=10 \mathrm{~K}$. 
dependence on $I$ and $H$. Again, these color plots represent phase diagrams, in which the critical current $I_{C}$ separates regions of high and low resistance states.

Just like in the field perpendicular geometry, $I_{C}$ clearly decreases with increasing external fields. This holds also true for the threshold currents, which indicate the onset of dynamic excitations. According to the arguments given in section 5.1.4, this anomalous field dependence suggests that even in very high in-plane fields the magnetization direction in the Co layers deviates from a perfectly parallel alignment with the field. In this way the FMR frequency and thus the critical currents might be reduced.
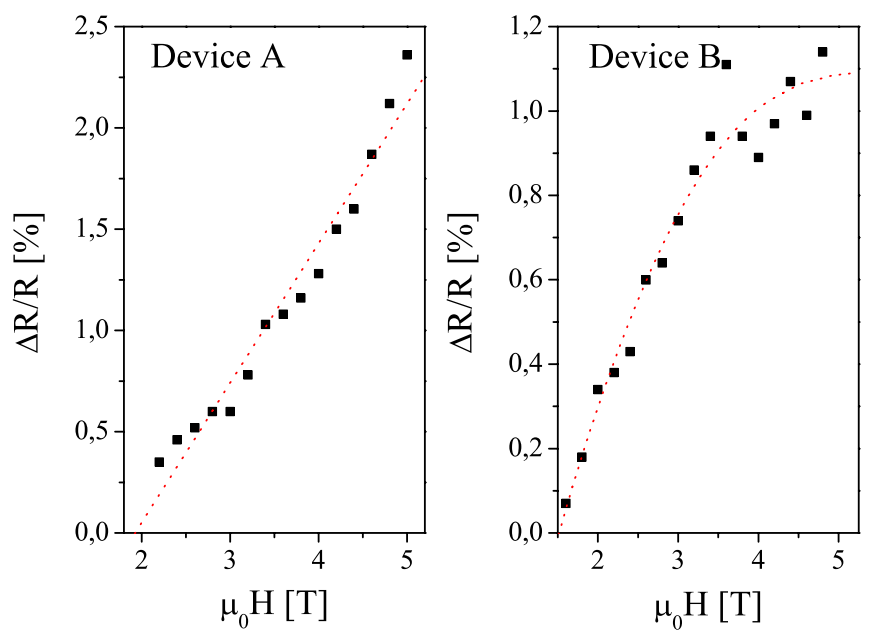

Figure 5.23: $\Delta R / R$ as a function of the external field for device $A$ and device $B$ in the field parallel to the plane geometry. The dotted red line is just a guide to the eyes.

The assumption that the magnetization is not fully saturated even in very high magnetic fields is further supported by the observed rise in the GMR values $\Delta R / R$ with increasing magnetic fields. The data displayed in figure 5.23 for device B (right panel) is taken at zero current bias, thus yielding information on $\left(R_{P}-R_{V o r t e x}\right) / R_{V o r t e x}$. In fields above the demagnetization field of $1.5 \mathrm{~T}$ the magnetizations of both Co layers are expected to lie in the sample plane in a perfectly parallel configuration. Since the GMR values are not constant in the very high field region, the latter assumption is not correct, but on the contrary there is strong evidence for the deviation of the magnetization from a perfectly parallel alignment with the external field. This is also demonstrated by the magnetoresistance loops measured at zero bias current, which are presented in section 5.4. A similar situation is observed for device $\mathrm{A}$. Here the increase in $\Delta R / R=\left(R_{A P}-R_{P}\right) / R_{P}$, which is assessed at the critical current $I_{C}$, is attributed to varying values for $R_{A P}$ : apparently the state of total antiparallel alignment is not reached 
for $\mu_{0} H \geq 1.5 \mathrm{~T}$, so that there is still a further resistance increase when the magnetization directions of the layers become more and more antiparallel. It can only be restated that the physics behind these peculiar magnetization configurations is not completely understood.

\subsection{Field Sweeps at Fixed Current Bias in the Field Parallel Geometry}

Because of the particular magnetization configuration within device $\mathrm{B}$, the consistency of current-induced excitations with field-induced effects is checked also in the field parallel to the plane geometry for device B. The corresponding results for the field sweeps at fixed current bias are displayed in figure 5.24 .

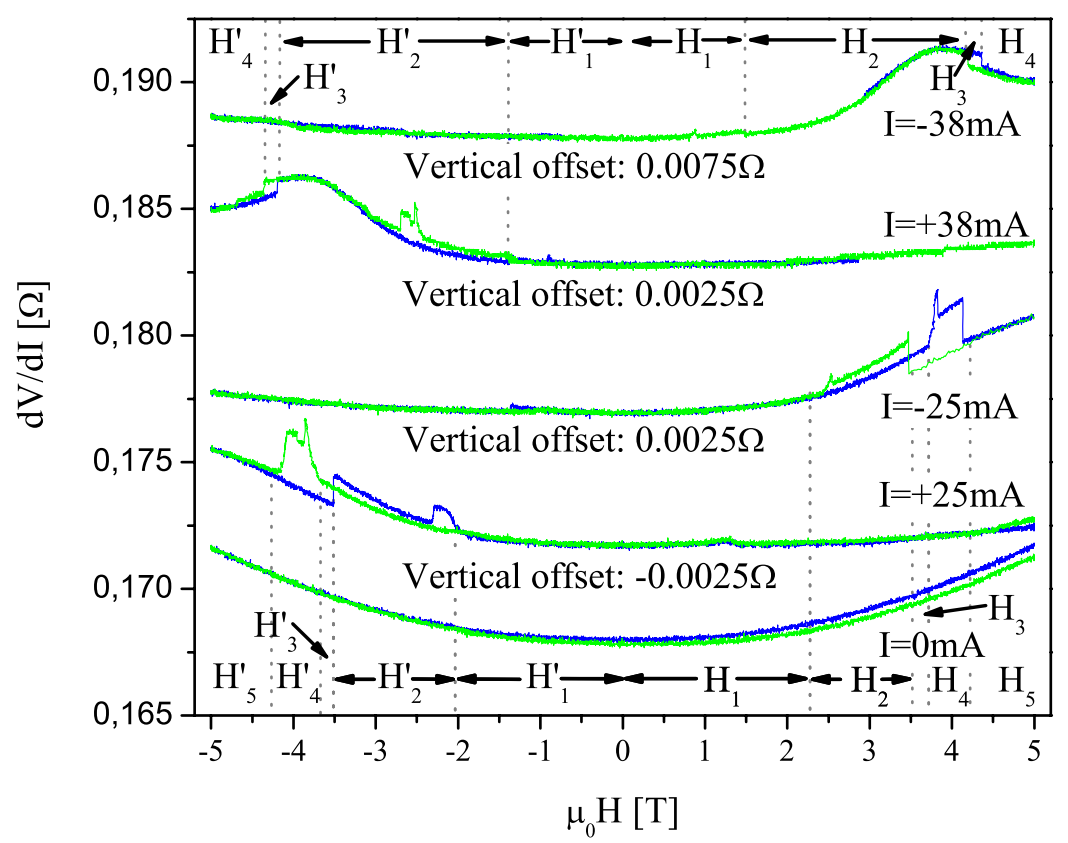

Figure 5.24: Field sweeps at $\mathrm{T}=10 \mathrm{~K}$ for the $3 \mathrm{nmCo} / 10 \mathrm{nmCu} / 12 \mathrm{nmCo}$ trilayer nanopillar (device $B$ ) in the field parallel to the plane geometry with a constant current bias of $0 m A,+25 m A,-25 m A,+38 m A$ and $-38 m A$, respectively. Blue lines indicate a sweep direction from $-5 T$ to $+5 T$, whereas the green curves refer to the opposite sweep direction.

The measurement at zero current bias shows a low resistance state at zero external field, but $d V / d I$ gradually increases with increasing external fields. This holds true for both field directions as well as for both sweep directions. 
The data recorded with a bias current of $-25 \mathrm{~mA}$ features a totally different curve progression; here the low resistance state is observed for negative and small positive field values, but above $\mu_{0} H=+2.2 \mathrm{~T}$ a gradual increase in $d V / d I$ is clearly visible. This increase is indicated by a peak structure followed by a high resistance plateau in the corresponding region $\mathrm{H}_{2}$ for sweeps from negative to positive field values (blue line). In the very high field regime $H_{3}$ the resistance is abruptly reduced, and hence the low resistance state (still superimposed by a gradual increase in $d V / d I$ ) is observed in the regions of $H_{4}$ and $H_{5}$. The measurements exhibit a clear hysteresis, i.e. the features described above are also detected for the opposite sweep direction towards negative field values (green curve), but here the threshold and critical fields are shifted to higher field values: the peak structure is found at a threshold field value of $\mu_{0} H=+3.7 \mathrm{~T}$, whereas the low resistance state is only observed in the regime of $H_{5}$.

The measurement performed at $I=-25 \mathrm{~mA}$ is reflected in the curves taken at opposite current polarity with $I=+25 \mathrm{~mA}$ : here the features detected within the field regimes $H_{2}-H_{5}$ for negative current bias are found for the inverse field direction within the regions $H_{2}^{\prime}-H_{5}^{\prime}$, whereas no effects are visible for positive and small negative field values. The effects are of similar form and magnitude, and also the absolute values of the threshold and critical fields approximately concur with the ones detected at $I=-25 \mathrm{~mA}$.

For even higher currents $(|I|=38 \mathrm{~mA})$ the magnetoresistance loops show a slightly different behavior. The curve recorded at $I=-38 \mathrm{~mA}$ also features a constant differential resistance for negative and small positive field values, but then the gradual increase in the regime $H_{2}$ is much steeper. Besides, no peaks and subsequent high resistance plateaus are measured in the very high field regions $H_{3}-H_{4}$. Instead, $d V / d I$ features a downward step at $\mu_{0} H=+4.2 \mathrm{~T}$ (blue curve) and $\mu_{0} H=+4.2 \mathrm{~T}$ (green curve), respectively. This abrupt reduction in $d V / d I$ is followed by a further gradual decrease for higher fields within the region $H_{4}$.

Again, the curves recorded at opposite current bias with $I=+38 \mathrm{~mA}$ represents the 'mirror image' of the measurement at $I=-38 \mathrm{~mA}$. However, one difference is thoroughly noticeable: there are a peak structure and a short subsequent high resistance plateau for the sweep direction towards negative values at $\mu_{0} H=-2.3 \mathrm{~T}$.

All of the results described above are discussed and compared to the current sweeps at fixed field values in the field parallel geometry within the following section 5.4.1.

\subsubsection{Field-Induced Effects versus Current-Induced Effects in the Field Parallel Geometry}

The field sweep at zero bias current agrees quite well with the observations for the current sweeps, which also indicate that $d V / d I$ increases gradually with increasing external fields above a certain field value (best seen in the color plots of figure 5.22 and in the right panel of figure 5.23). The low resistance state is ascribed to the vortex state within the 'fixed' Co layer, 
while the 'free' layer is most likely in a single domain state with the magnetization lying in the plane. For higher magnetic fields the vortex core moves from the center towards the edge of the Co layer, thus causing a gradual rise in $d V / d I$. Yet, for field values above the demagnetization field of the $12 \mathrm{~nm}$ Co layer the vortex should have disappeared and both layers should have reached complete parallel alignment. But this is obviously not the case, since the resistance further increases with increasing field. The reasons for this anomaly are not really understood. The measurements performed at a current bias unequal to zero also essentially reflect the results obtained for the current sweeps. For the magnetoresistance loops with $I=-25 \mathrm{~mA}$ the current stabilizes the vortex state for negative magnetic fields, but in positive magnetic fields the gradual transition to parallel alignment is clearly visible. The peak structure and the subsequent high resistance plateau are ascribed to uniform excitations in the thin Co layer, which lead to an increase in GMR. At $I=-38 \mathrm{~mA}$ these dynamic excitations are not observed anymore, but the current is now sufficient to stabilize the vortex state even at high positive magnetic fields: in accordance with the interpretation of the current sweeps the downward step is attributed to the nucleation of a vortex core. Only the resistance peak in the $I=+38 \mathrm{~mA}$ magnetoresistance loop at $\mu_{0} H=-2.4 \mathrm{~T}$, which is ascribed to further uniform precessional modes, cannot be identified in the corresponding region of the current sweeps.

In conclusion, the phase boundaries between states of higher and lower resistance deduced from the current sweeps essentially seem to confirm the results of the field sweeps - just like in the field perpendicular geometry. Again, this also applies for the observed hysteresis as well as for the emergence of most uniform excitations. However, the field sweeps are not symmetric around zero; this anomalous behavior has already been found in the measurements performed in the field perpendicular geometry (cf. section 5.2.1). Once more this implies a much more complicated magnetization configuration than the one described above. The reasons for the deviations from the single domain behavior even for extremely high applied fields can only be assumed to be hidden in the complex interplay between the MaxwellOersted field and the external magnetic field.

\subsection{Discussion and Summary for the Trilayer Samples}

The presented transport data for the trilayer devices A and B give reveal the emergence of current-induced excitations at high DC current densities and in large external fields - this applies for the in-plane as well as for the out-of-plane geometry. For both samples the characteristic peak structures are attributed to uniform precessional states, whereas the hysteretic changes in $d V / d I$ result from static changes in the magnetization configuration of the $3 \mathrm{~nm}$ thin Co layer. In the most simple semi-classical spin torque model at least some of these changes can be attributed to current-induced switching processes of the $3 \mathrm{~nm}$ thin Co layer between two different single domain states: the parallel (low resistance) and the antiparallel (high re- 
sistance) configuration with respect to the $12 \mathrm{~nm}$ thick Co layer. However, the absence of excitations for field values below the demagnetization field of $\mu_{0} M_{S} \approx 1.5 \mathrm{~T}$ indicates that also vortex states play a major role for the interpretation of the data, i.e. a simple macrospin model does not seem to be sufficient in order to explain all observed effects. This is supported by the existence of further low resistance states, which are ascribed to the nucleation of vortices due to the influence of the Maxwell-Oersted field, in the field parallel to the plane geometry for device B. Also the field sweeps show that the actual magnetization configuration within the Co layers is much more complex and not fully aligned with the field even in higher magnetic fields (asymmetry for high current densities, no saturation for higher external fields at zero bias current). Nevertheless these magnetoresistance loops show a good correspondence with the current sweeps. From the combination of these two measurement techniques the phase diagrams depicted in figure 5.25 are deduced. The sketches within the diagrams represent some possible magnetization configurations for the corresponding field and current region, respectively. Here also excitations for positive current bias are visible; most likely these features are related to excitations in the $12 \mathrm{~nm}$ thick Co layer.

However, the semi-classical spin torque model described in section 2.7.2 does not capture all general features observed in the data. Since the transport measurement technique applied throughout this thesis provides only indirect evidence for static and especially dynamic excitations, the nature of possible motions remains unclear. For this reason measuring the frequency spectra of the resulting microwave power might give further insight into the process of spin-transfer-driven switching for devices A and B. Such experiments have already been performed on similar sample structures [70, 75]. Moreover, time-resolved imaging of the reversal process by means of ultrafast x-ray microscopy as it has been conducted by Acremann et al. [76] reveals the exact evolution of the magnetization under the influence of a DC current, allowing for nanometer spatial resolution. Due to the elementsensitivity of the XMCD technique, this method could even provide further information on the spin accumulation patterns in the normal metal by measuring the induced magnetic moment per $\mathrm{Cu}$ atom. Hence, the fundamental roles of the torques produced by charge and spin currents could be explored further and the development of a model beyond the macrospin concept might be possible. 

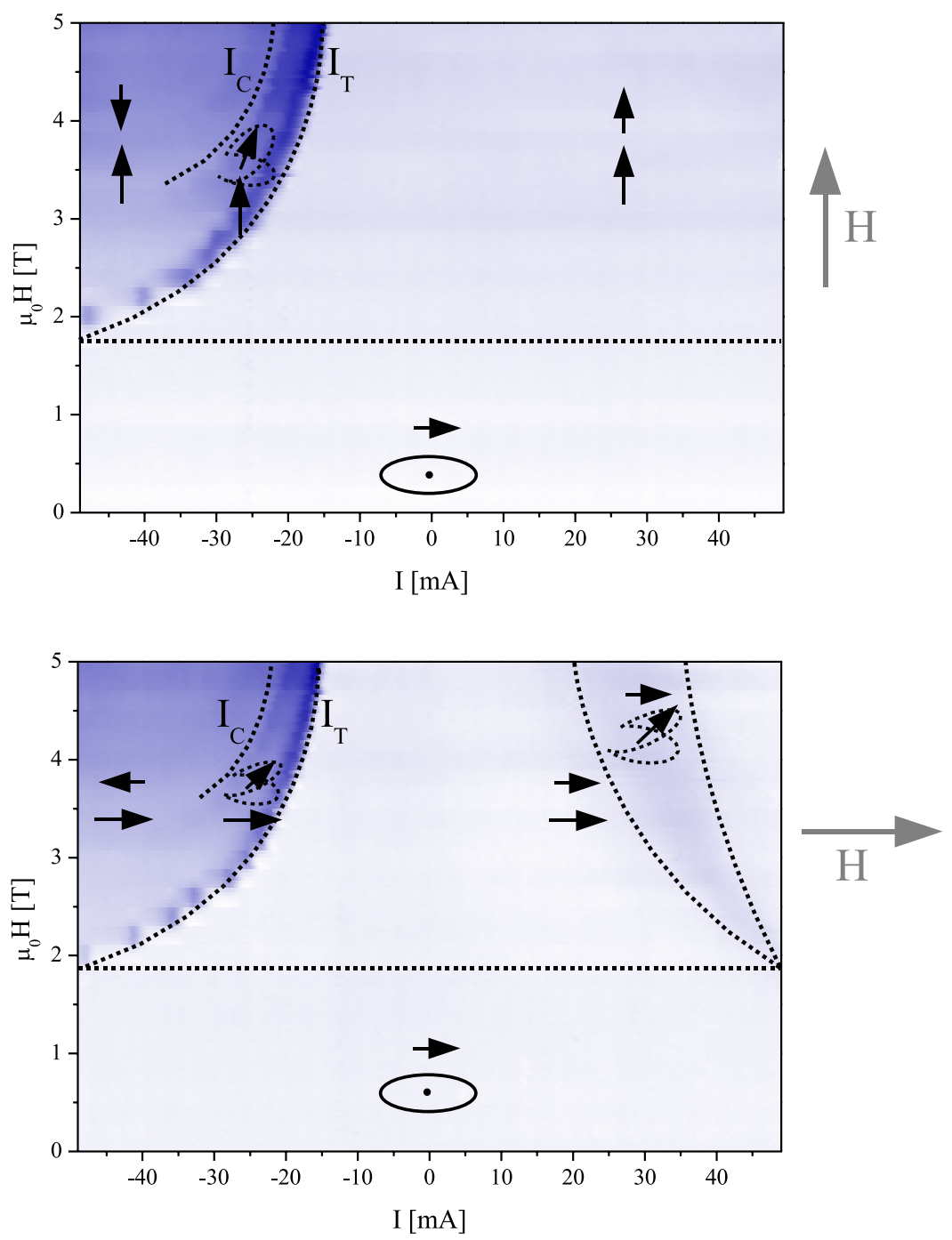

Figure 5.25: Simplified phase diagram for some possible magnetization configurations within the $3 \mathrm{~nm}$ Co layer (short arrows) and the 12nm Co layer (longer arrows) of the trilayer device A. The top panel depicts the field perpendicular to the plane geometry, whereas the bottom panel displays the results for the field lying in the sample plane. As mentioned in the text, there are many indications for the existence of more complex magnetization configurations than the ones shown here. 

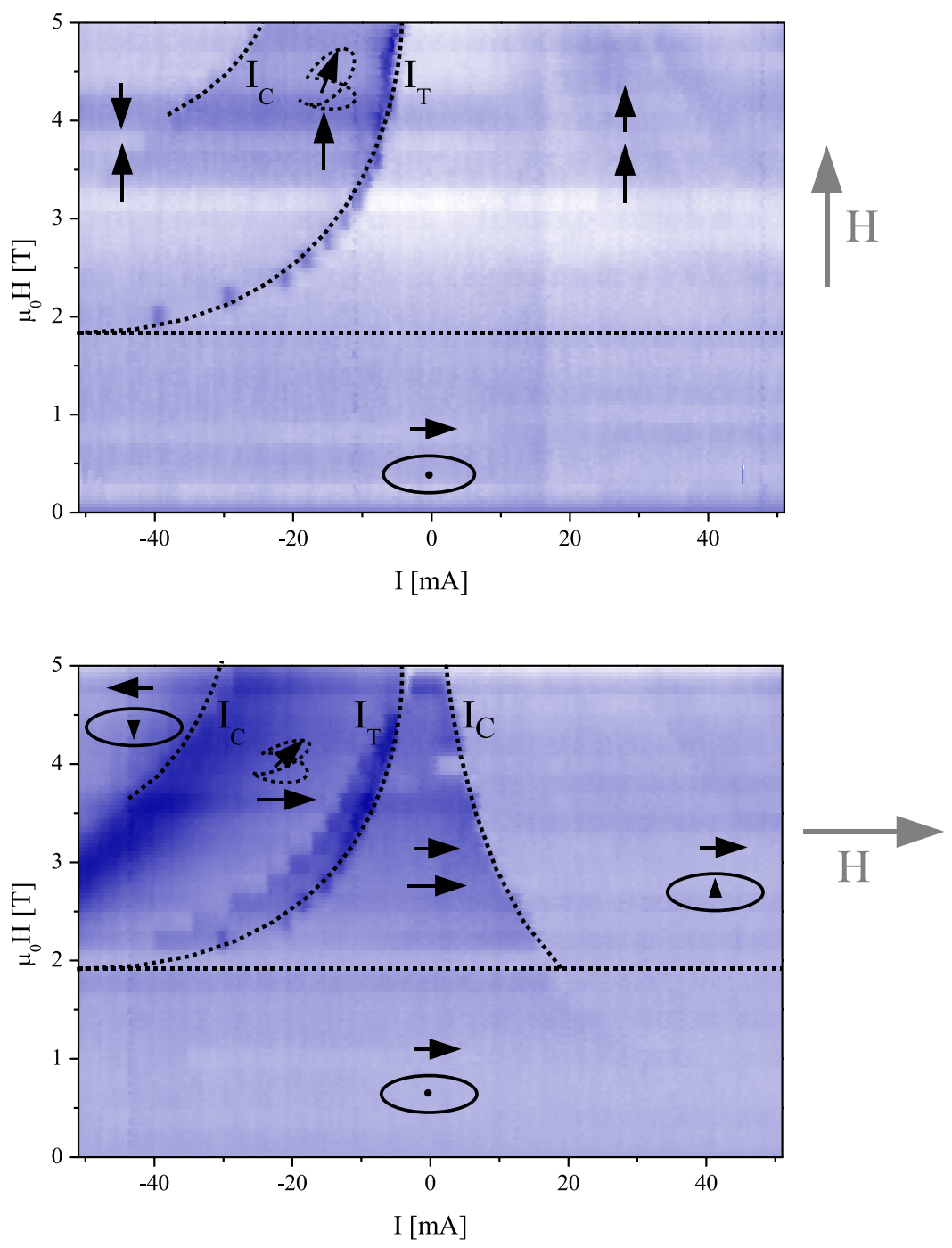

Figure 5.26: Simplified phase diagram for some possible magnetization configurations within the $3 \mathrm{~nm}$ Co layer (short arrows) and the 12nm Co layer (longer arrows) of the trilayer device B. The top panel depicts the field perpendicular to the plane geometry, whereas the bottom panel displays the results for the field lying in the sample plane. Again, there are many indications for the existence of more complex magnetization configurations than the ones shown here. 


\section{CHAPTER 6}

\section{Current-Induced Excitations in Ferromagnetic Single Layer Samples}

In the previous chapter it has been shown that the presence of a high current density has a significant influence on the magnetoresistance of a ferromagnetic trilayer system. Within this chapter current-induced excitations in ferromagnetic single layer devices are analyzed. Based on the model introduced in section 2.8 the spin-filtering properties of an asymmetric single layer are examined, where the asymmetry in spin accumulation is achieved by adding an additional layer of $\mathrm{Pd}$ close to one of the interfaces of the Co layer.

Hence, the layer sequence is $3 \mathrm{nmCu} / 5 \mathrm{nmPd} / 5 \mathrm{nmCu} / x \mathrm{nmCo} / 30 \mathrm{nmCu}$ with $x=6,8,10$ and 12 , respectively. Again, several samples were studied, but here representative data for only four of them, i.e. one data set for each sample thickness, is displayed. The most intriguing features of these measurements are exemplified by discussing the data for the $6 \mathrm{~nm}$ and the 8nm Co device more explicitly. Just like for the trilayer experiments both current sweeps at fixed applied fields and field sweeps at fixed current bias were performed in the field in-plane and the field out-of-plane geometry for field values up to $5 \mathrm{~T}$. These were completed by a few measurements in extremely high fields up to 9T. Positive current bias is defined such that the electrons flow through the Co film before entering the Pd layer (see inset of figure 6.2). Sweeping times for the DC current were approximately $d I / d t=0.3 \mathrm{~mA} / \mathrm{s}$, while the field was swept at a rate of $d B / d t=4 \mathrm{mT} / \mathrm{s}$. The differential resistance was measured with an amplitude of $100 \mu \mathrm{A}$ at a frequency of $f=7013 \mathrm{~Hz}$. All measurements (except for the temperaturedependent ones presented in the last section) were carried out at $T=10 \mathrm{~K}$.

Due to the absence of a second ferromagnetic layer, the GMR effect cannot be utilized anymore for detecting current-induced excitations of the magnetization. However, Özyilmaz et al. demonstrated that the magne- 
toresistance of a single ferromagnetic layer is sufficient to resolve changes in the relative orientations of the magnetization directions within this layer - despite the small size of the effect of approximately $\Delta R / R \approx 1 \%[20]$. This order of magnitude is still within the reach of the developed transport measurement setup. However, also the influence of the classical anisotropic magnetoresistance effect has possibly to be taken into account; this issue is addressed in subsection 6.2.1.

\subsection{Current Sweeps at Fixed Applied Fields in the Field Perpendicular Geometry}

Figure 6.1 summarizes the results of the current sweeps in the field perpendicular to the plane geometry for the four single layer devices. Just like for the trilayer devices no excitations are detected in zero external field, so that these curves are subtracted from each current sweep in order to eliminate the parabolic background resistance. Thus the color plots once more represent phase diagrams, in which the threshold or the critical currents $\left(I_{T}\right.$ and $I_{C}$, respectively) mark the boundaries between different states with varying relative changes in the orientation of the magnetization within the single Co layer.

The color plots reveal that excitations can be observed for all Co layer thicknesses in sufficiently large magnetic fields higher than $\mu_{0} M_{S} \approx 1.5 \mathrm{~T}$. ${ }^{1}$ However, the changes in the differential resistance differ significantly in shape: there are dips, smaller peaks and upward as well as downward steps in $d V / d I$. The latter features can be hysteretic when observed for positive current bias, but also reversible steps for the opposite current polarity are detected (most obvious for the $6 \mathrm{~nm}$ device). Generally speaking the most distinctive excitations are found for positive current bias, whereas changes in $d V / d I$ for negative current bias can essentially only be noticed in extremely high magnetic fields above $3 \mathrm{~T}$.

All the features listed above are described in greater detail and discussed separately in the following subsections. Also the questions of the static or dynamic excitations (subsection 6.1.3) and the specific polarity dependence are considered. In the subsequent subsection 6.1.5 the field behavior of the threshold and critical currents is addressed; finally also in terms of their thickness dependence (subsection 6.1.6).

\subsubsection{Decrease of the Single Layer Resistance}

Figure 6.2 displays a typical example of a current-induced decrease in sample resistance as it is observed for the $6 \mathrm{~nm}$ Co single layer in large applied fields: at $I_{C 1}=+15.6 \mathrm{~mA}$ (sweep towards positive current bias) and $I_{C 2}=+7.5 \mathrm{~mA}$ (opposite sweep direction) a downward step with $\Delta R / R \approx 0.9 \%$ is detected

\footnotetext{
${ }^{1}$ The $10 \mathrm{~nm}$ single Co layer is an exception, because here a gradual decrease followed by a gradual increase in $d V / d I$ is identified also in the low magnetic field regime. Since this effect appears for both current polarities, it is most likely not current-induced, but it constitutes an outlier due to the influence of the Maxwell-Oersted field.
} 

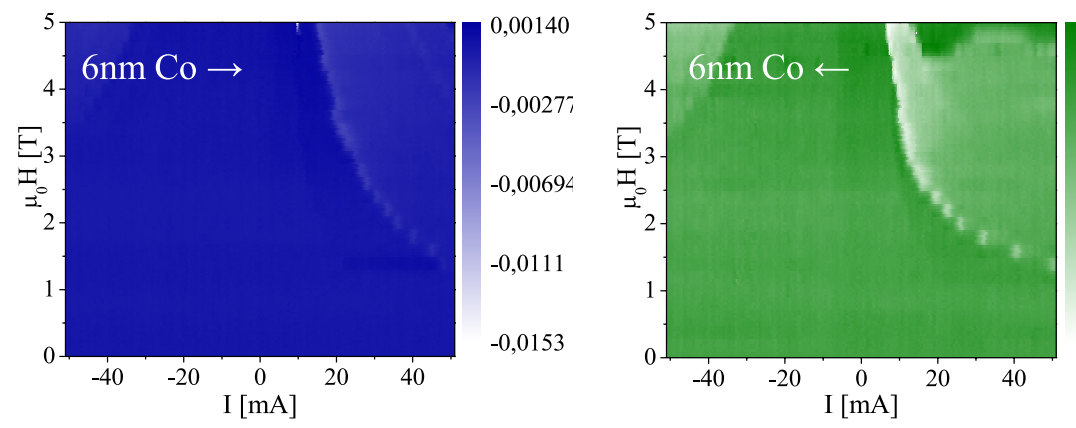

0,00108
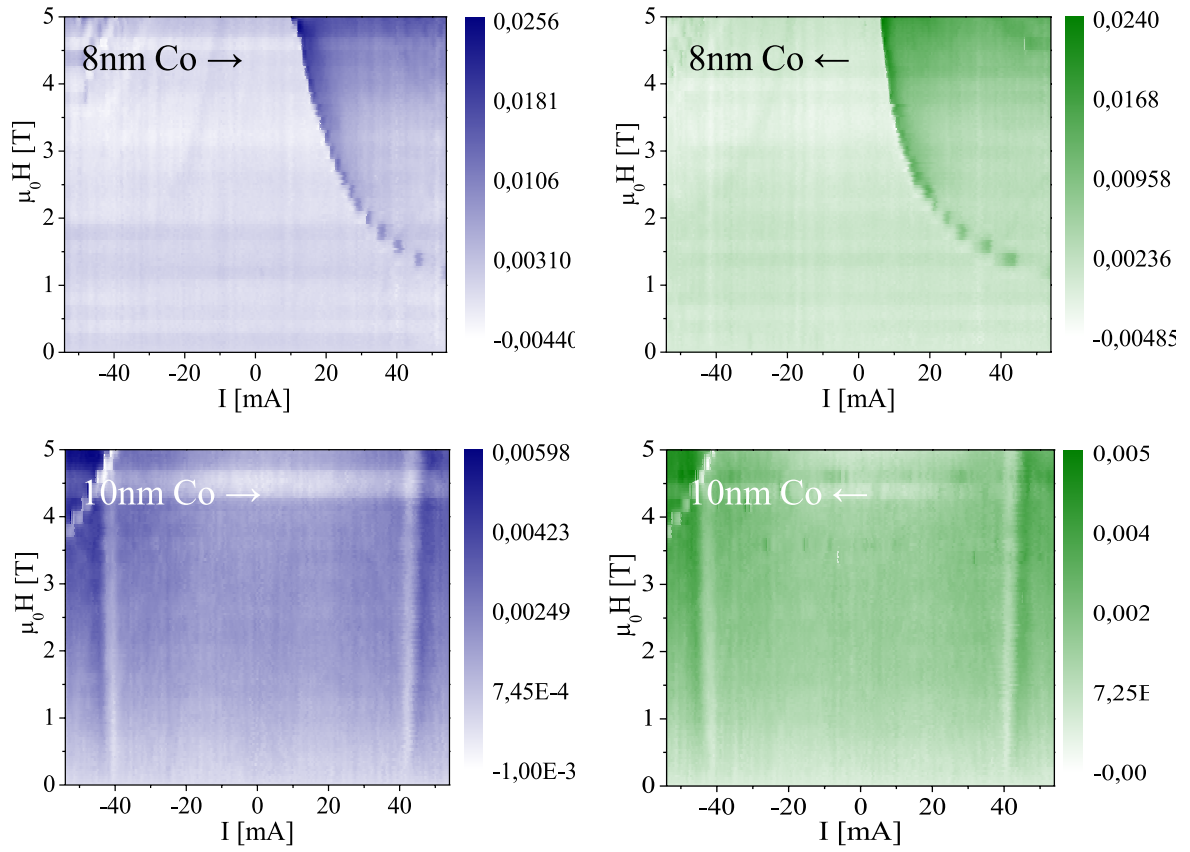

0,005
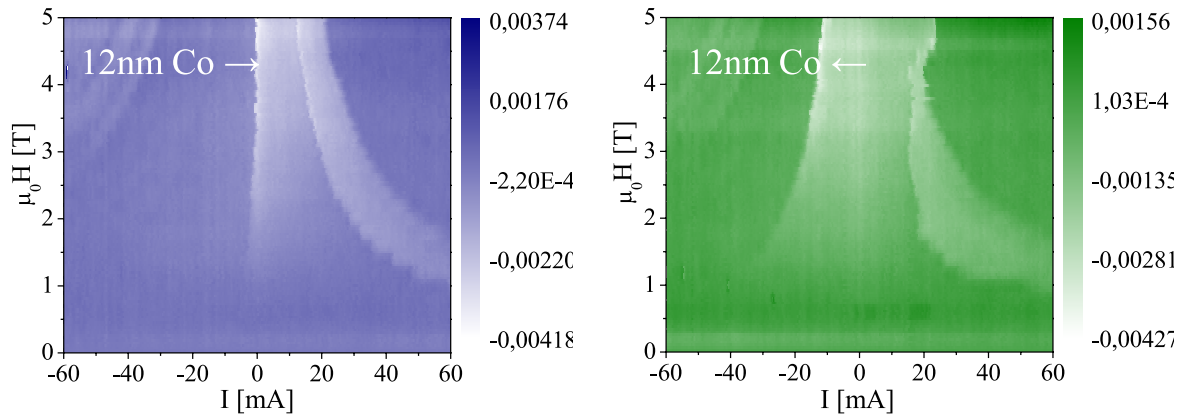

Figure 6.1: Color plots for $d V / d I$ as a function of $I$ and $H$ in the field perpendicular to the plane geometry. The corresponding Co layer thicknesses and the current sweep directions are indicated in the graphs. 
in $d V / d I$. Moreover, there is an additional reversible decrease at negative currents with a threshold of $I_{T}=-37.7 \mathrm{~mA}$. Similar downward steps and also dips in $d V / d I$ can be identified in the data sets of the other Co single layers as well.

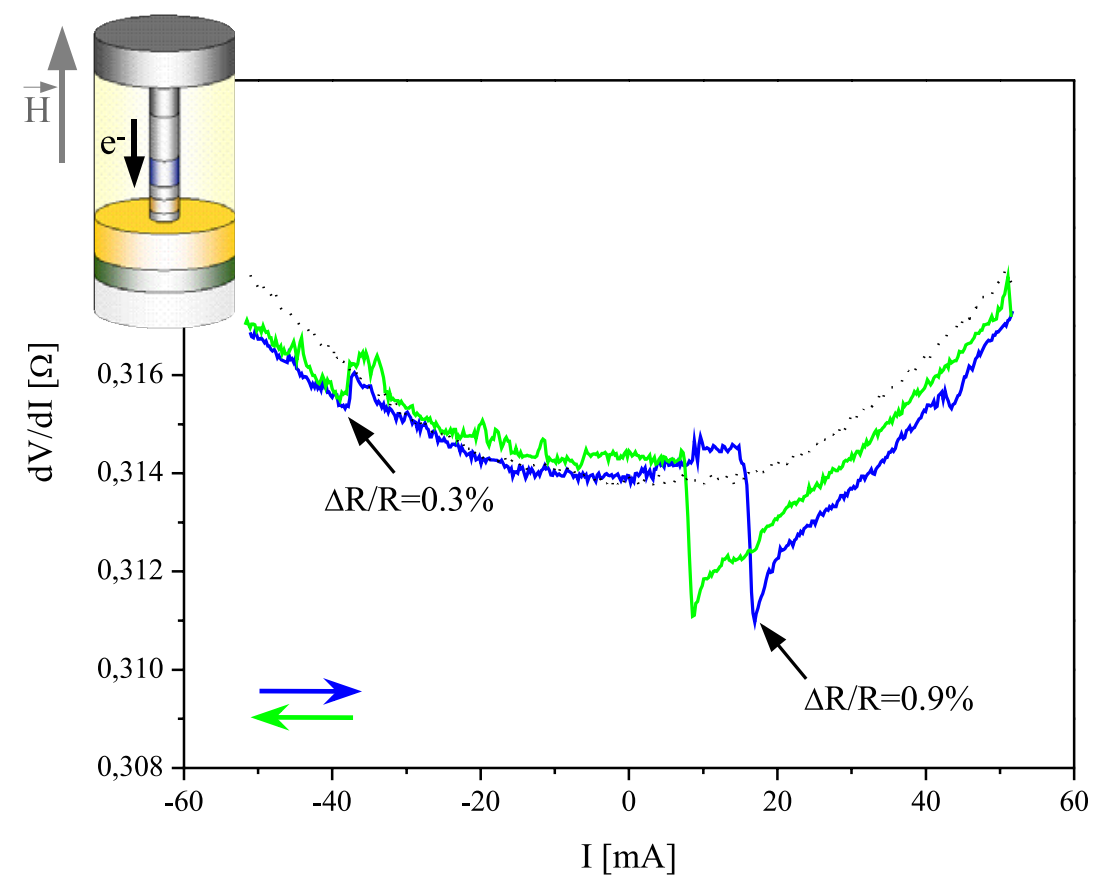

Figure 6.2: Current sweep for the 6nm thick Co single layer device in the field perpendicular to the plane geometry at $B=4.4 T$ and $T=10 \mathrm{~K}$. The inset displays the direction of positive current flow and the corresponding magnetic field direction.

Based on the findings for the trilayer samples, also the single Co layer is very likely in the vortex state if there is no or just a small magnetic field. Thus, the applied field has to be high in order to create a significant spin accumulation at the $\mathrm{Co} / \mathrm{Cu}$ interfaces, because for the vortex configuration the spin accumulation is considerably reduced due to the spin diffusion along the interface (cf. section 5.3.1). This also explains the absence of any excitations in fields below the demagnetization value of $\mu_{0} M_{S}=1.5 \mathrm{~T}$. Here two alternative models, which both might account for the detected decreases in $d V / d I$, will be briefly described.

The first one assumes that in fields above $\mu_{0} M_{S}=1.5 \mathrm{~T}$ the magnetization of the Co layer is directed completely out of plane. Then one spin channel is reflected at the $\mathrm{Cu} / \mathrm{Co}$ interfaces, hence blocked, and a spin accumulation pattern emerges at both interfaces. Because of the Pd spin diffuser close to one of these interfaces, the spin accumulation is asymmetric. Hence a net torque arises, and at high current densities this torque is sufficient to 
(locally) change the magnetization configuration in the Co. As stated before, a transverse inhomogeneity in the magnetization can effectively reduce the spin accumulation and might thus lead to a reduction of the differential resistance. This is the case if two opposite magnetization directions are present in the Co film, where one of them is pointing parallel to the $B$ field and the other one is exactly antiparallel (see figure 6.3 (a)). In the two resistor model described within section 2.8 the decrease in $d V / d I$ corresponds to the opening up of a lower resistance channel in parallel.

According to the comments of the last paragraph an increase in $d V / d I$ should be identified in the data upon comparing the low field resistance (vortex state) to the high field resistance (close to uniform magnetization configuration) for zero DC current bias. However, this change in the differential resistance is not noticeable in any of the data sets of figure 6.1 when the magnetic field is increased above $1.5 \mathrm{~T}$.

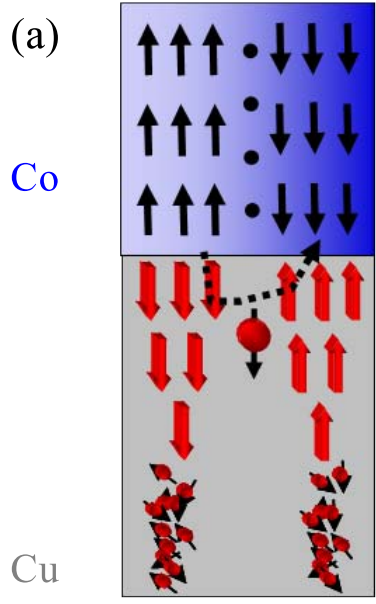

(b)

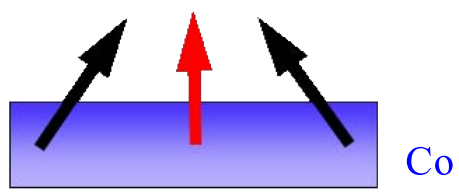

Parallel, high resistance
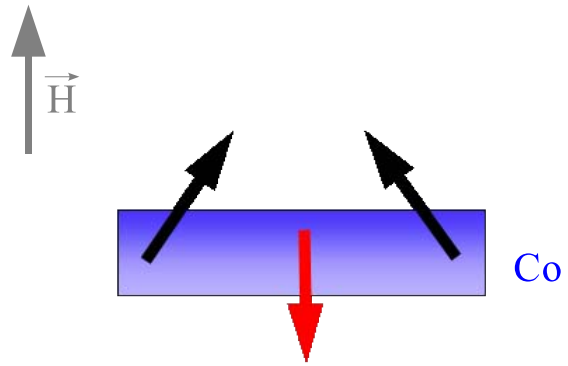

Antiparallel, low resistance

Figure 6.3: (a) Most simple model for the reduced interface magnetoresistance. (b) More specific magnetization configuration (vortex state) leading to a decrease in interface magnetoresistance.

Hence, a more specific magnetization configuration is considered in figure 6.3 (b): here the ideas first developed by Özyilmaz [20] are adapted to the circular geometry of the Co layers prepared within this thesis work. Again, the ground state of the Co film in the single layer device is a vortex configuration. By applying a magnetic field perpendicular to the film plane, the vortex core is dilated with increasing out-of-plane field (see upper panel of figure $6.3(\mathrm{~b})$ ). For this model it is assumed that the outer part of the vortex represented by the black arrows is fixed, whereas the inner part marked by the red arrows is supposed to be flexible. With increasing fields, the magnetization turns more and more out of plane. At the same time the arising spin torque acts on the flexible inner part of the vortex 
and at sufficient current densities the magnetization is eventually switched into an antiparallel configuration of lower resistance (lower panel of 6.3 (b)). It is remarkable that in the case of the $12 \mathrm{~nm}$ Co device the ferromagnetic layer stays in the metastable low resistance state even for zero current bias. Here the vortex might be stabilized by the reduced dipolar field in this configuration. Apparently a macrospin model does not suffice for the single layer geometry. While the vector model for two distinct regions (outer part and inner part of the vortex) as described above is only the simplest way to address the problem, a far more elaborate method to study the accessible modes in such a structure are micromagnetic simulations. These have been performed elsewhere [77] for very similar devices. The corresponding results are discussed in the following section, where also the possible reasons (including the simulated mode spectra) for an increase in $d V / d I$ are specified.

\subsubsection{Increase of the Single Layer Resistance}

Some of the devices show also a distinct increase in the differential resistance at sufficient current densities. The best example is given by the $8 \mathrm{~nm}$ single layer, whose data is presented in figure 6.4. At $I_{C 1}=+9.3 \mathrm{~mA}$ (sweep direction from negative to positive current bias) there is an upward step in $d V / d I$, which is hysteretic and hence reappears at $I_{C 2}=+5.6 \mathrm{~mA}$ for the opposite sweep direction. The increase in the differential resistance roughly amounts to $1.4 \%$. For the opposite current polarity there are also some irregular and less distinct increases in $d V / d I$ at $I_{T}>37 \mathrm{~mA}$.

In accordance to the explanations of the last section, a decrease in $d V / d I$ corresponds to a lower resistance channel which opens up in parallel to the higher one. However, an increase in resistance then indicates an additional resistance in series. Regarding the magnetization configuration within the Co layer this means that there exists a gradual rotation of the magnetization along the direction of current flow - very similar to the magnetization configuration within a domain wall. A descriptive sketch of this situation is shown in figure 6.5. The gradual rotation has to be within the spin relaxation length in order to produce a significant rise in $d V / d I$ (adiabatic limit) [78].

In reference [77] micromagnetic calculations are performed in order to determine the precession patterns of some of the lowest-frequency magnetic normal modes of a Co disk. This disk with a diameter of $50 \mathrm{~nm}$ and a thickness of $15 \mathrm{~nm}$ is perpendicularly magnetized, and it is excited by a locally strongly inhomogeneous field pulse. The resulting simulated power spectra as well as the mode images are depicted in figure 6.6. In addition to the lateral inhomogeneity (transverse to the direction of current flow), the mode spectrum in reference [77] is classified by an even and an odd mode along the $z$ axis (longitudinal to the current flow). According to the results of [43] the lowest threshold current is expected for a mode odd in $z$ and with a wavelength comparable to the one of the device diameter. This is the second odd mode marked by the red frame in figure 6.6. In 


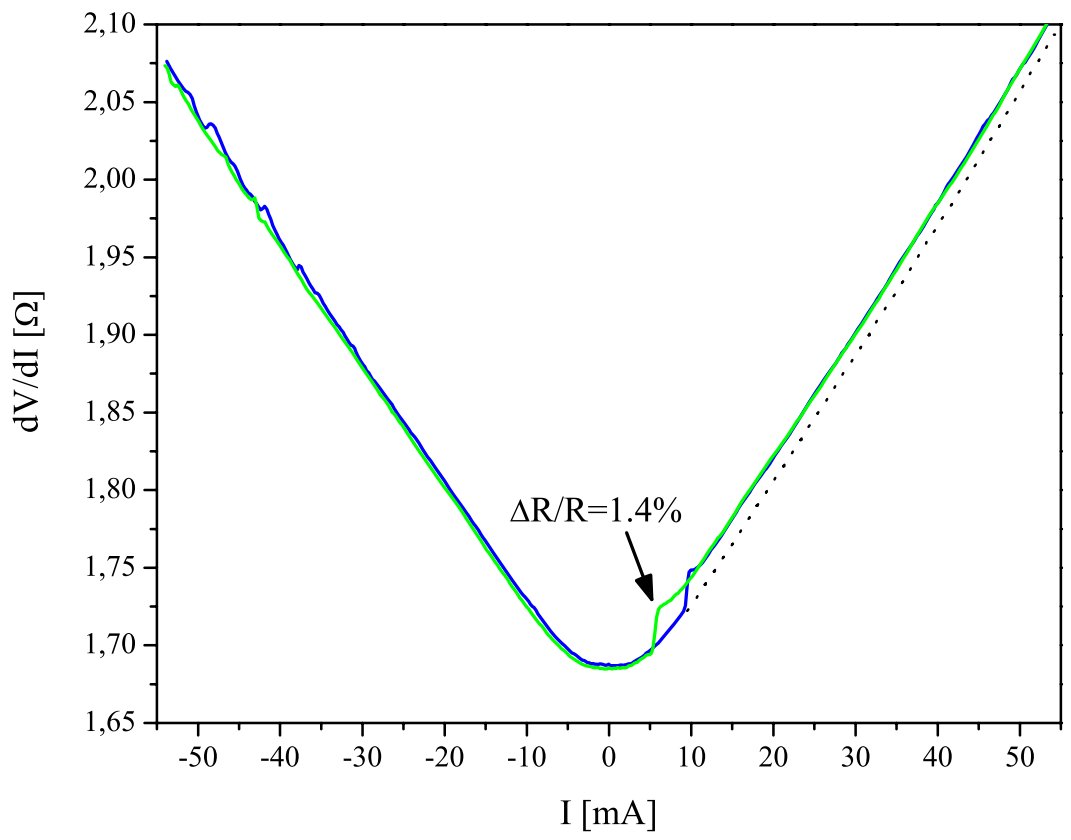

Figure 6.4: Current sweep for the 8nm thick Co single layer device in the field perpendicular to the plane geometry at $B=5 T$ and $T=10 \mathrm{~K}$.

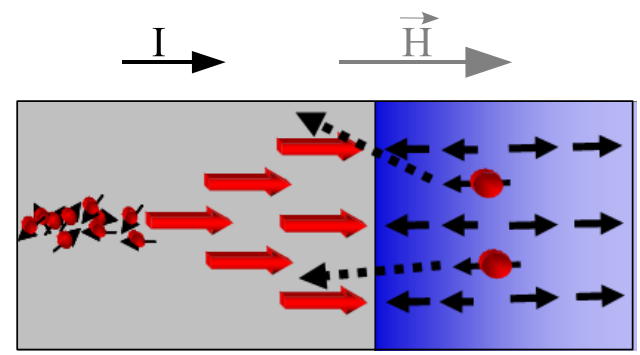

Figure 6.5: Schematic illustration of a resistance increase due to an inhomogeneous magnetization configuration along the direction of current flow.

spite of other modes having a lower frequency and hence less damping, the greater current-induced torque because of the asymmetry in the $n_{z}=1$ mode provides the lowest threshold. The excitation of a similar mode is also very likely for the samples discussed within this chapter, and thus the explanation of the observed increase in $d V / d I$ in terms of an inhomogeneity along the direction of current flow is thoroughly plausible. 


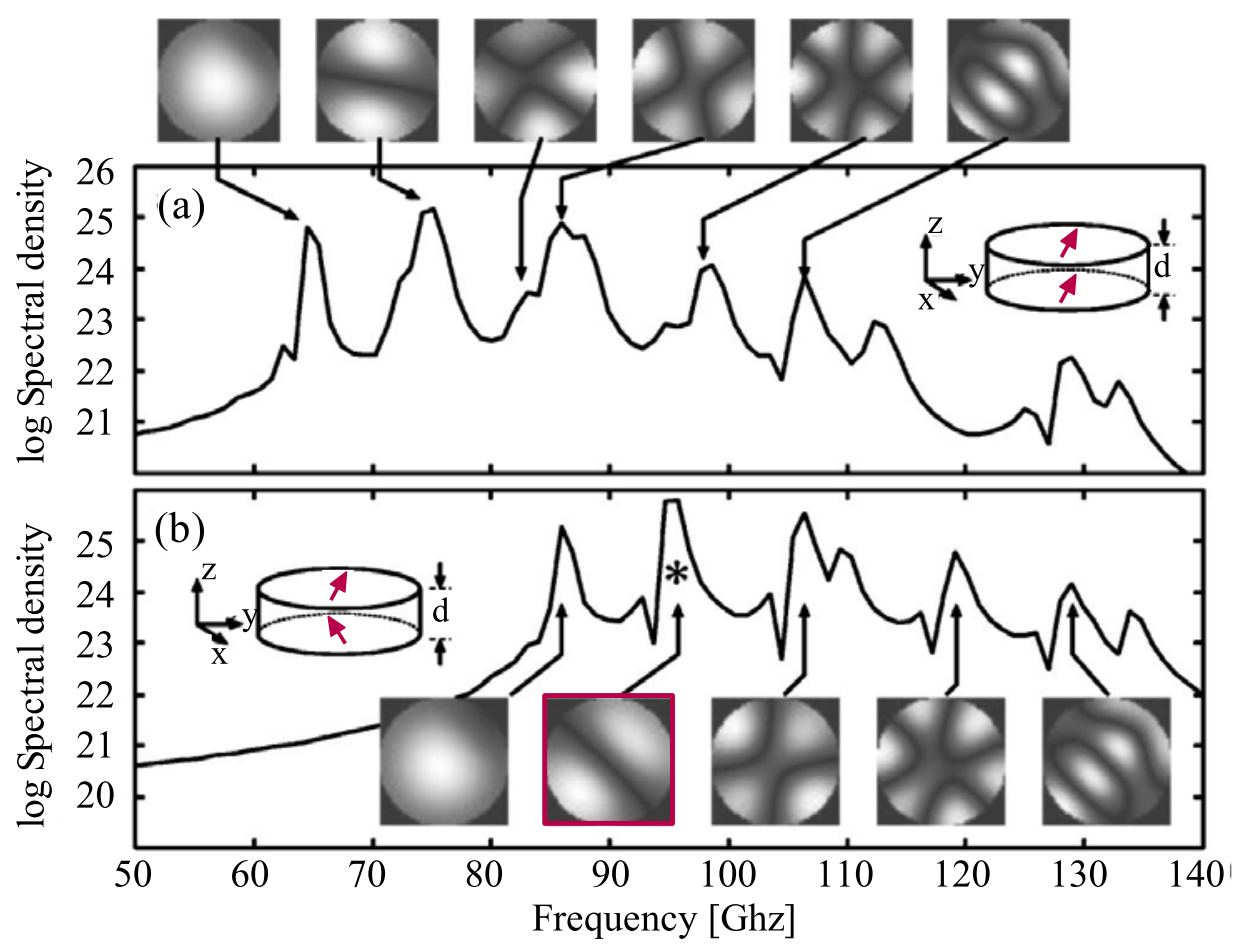

Figure 6.6: Eigenmodes of a 15nm thick, 50nm wide Co disk magnetized perpendicular to the plane: (a) modes with even z symmetry, (b) modes with odd $z$ symmetry. Since the odd mode marked by the red frame is nonuniform both in plane and normal, it is most likely to be driven unstable by a spin current. Taken from [77].

\subsubsection{Static Excitations versus Dynamic Excitations}

In the discussion for the trilayer devices in the previous chapter it has already been pointed out that the transport measurements do not allow for a direct determination between static and dynamic excitations. However, some indirect conclusions from the single layer data on the nature of the current-induced excitations are possible.

The observation of a hysteretic behavior for at least some of the changes in $d V / d I$ suggests the existence of a static bistable state with two different resistance values - just like for the trilayer geometry. On the other hand the measurements in very high fields with $\mu_{0} H>5 \mathrm{~T}$ prove that for all single layer devices, which show a change in $d V / d I$ in the field regime below $5 \mathrm{~T}$, excitations are also observed even at $\mu_{0} H=9 \mathrm{~T}$. As an example the high field behavior of the $8 \mathrm{~nm}$ thick Co single layer is depicted in figure 6.7. This makes a static state quite implausible. Besides, if a static non-uniformity differs from the rather unlikely magnetization configuration depicted in figure 6.3, the precessional term $\vec{M} \times \vec{H}$ of the Landau-Lifshitz-Gilbert equation should rapidly lead to a current-induced dynamic state.

Although the question of static or dynamic excitations can apparently 


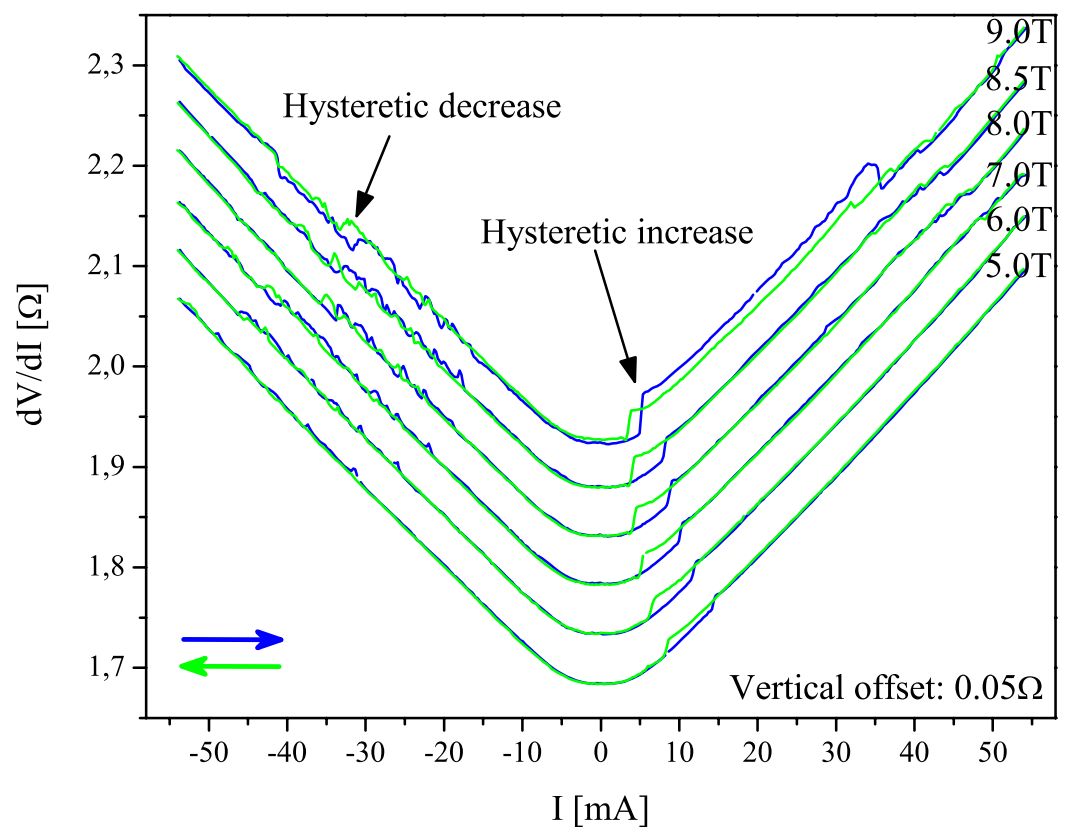

Figure 6.7: Current sweeps for the 8nm thick Co single layer device in the field perpendicular to the plane geometry at very high fields with $B>5 T$ and at $T=10 \mathrm{~K}$.

not be completely solved here, there are a few things that can be clarified about the (non-hysteretic) dynamic excitations. In analogy to the findings for the trilayer samples, the dips in $d V / d I$ give strong evidence for nonuniform dynamic excitations, because the excitation of coherent spin waves is not expected to effectively reduce the spin accumulation. Also the peaks or reversible upward steps in the differential resistance have to be directly related to non-uniform dynamic excitations: due to the discussion of the micromagnetic simulations in reference [77] in connection with figure 6.5 within the previous section, it is clear that the excitation of a mode with odd $z$ symmetry leads to an increase in $d V / d I$.

\subsubsection{Polarity Dependence of Single Layer Excitations}

Looking back on the overview color plots in figure 6.1 reveals that almost all excitations are observed for positive current polarity, i.e. for electrons flowing through the Co layer before impinging on the $\mathrm{Pd}$ layer. This underlines the importance of the $\mathrm{Pd}$ spin diffuser, which introduces strong spin flip scattering at the $\mathrm{Pd} / \mathrm{Cu}$ interface. In this way the spin accumulation at the $\mathrm{Cu} / \mathrm{Co}$ interface facing the $\mathrm{Pd}$ layer is effectively reduced, and an asymme- 
try in the spin accumulation pattern is generated. For positive current bias the spin accumulation at the $\mathrm{Cu} / \mathrm{Co}$ interface can thus exert an effective torque on the Co magnetization, which tends to amplify non-uniformities in the magnetization (figure 2.16 (b)). Hence, changes in $d V / d I$ are detected. For opposite current flow the resulting torque changes sign, which means that the torque suppresses deviations away from the average magnetization direction (figure 2.16 (c)). This unipolarity of the excitations in high magnetic fields has been confirmed by Ji et al. [7] in point contact experiments as well as by Özyilmaz et al. [9] for the single layer nanopillar geometry.

Still, figure 6.1 also denotes some smaller excitations for negative current bias - especially for extremely high currents with $|I|>30 \mathrm{~mA}$. According to the theoretical model of Stiles et al. [43] this is an indication for variations of the magnetization along the direction of current flow, which cause excitations for both polarities. Such transverse inhomogeneities have already been discussed in section 6.1.2, where it was shown that they cause an increase in the differential resistivity. However, the data sets for the $6 \mathrm{~nm}$ and the $12 \mathrm{~nm}$ Co layers rather show a decrease in $d V / d I$ at negative current polarities, whereas only the measurements for the $8 \mathrm{~nm}$ and the $10 \mathrm{~nm}$ thick samples display an increase (peaks) in $d V / d I$. The origins of this unexpected behavior are not fully understood.

\subsubsection{Field Dependence of Single Layer Excitations}

The color plots in figure 6.1 also allow for a good overview of the applied field dependence of the excitations within the single Co layers. A general tendency for the critical or threshold currents in higher fields is clearly visible: $I_{C}$ or rather $I_{T}$ decrease non-linearly with increasing fields. This holds true for both positive and negative current bias. The only exception is the behavior of $I_{T}$ for the $12 \mathrm{~nm}$ Co layer at high fields above $4 \mathrm{~T}$, where for the sweep direction from positive to negative current bias (lower right panel of figure 6.1) an increase of $I_{T}$ with increasing fields can be identified (at positive current values).

The latter high field behavior is also expected from other experimental studies [7, 9] as well as from theoretical models [43]. The anomalous critical current tendency for the remaining single layer data here is very similar to the findings for the trilayer devices discussed in the previous chapter. According to reference [20] the field behavior of the threshold current essentially depends on the amplitude of the excitations relative to the angle $\Theta_{\max }$ between two neighboring magnetization directions, at which the maximum spin-transfer torque is exerted. For the perpetuation of small amplitude non-uniform modes with $\Theta<\Theta_{\max }$ a decrease of the angle between local magnetization directions results in a reduction of the net spin torque, because $\Theta \rightarrow 0$ and hence away from $\Theta_{\max }$. For this reason an increasing external field, which effectively reduces the angle $\Theta$ between two neighboring magnetization directions, yields an increase of the threshold current $I_{C}$. This situation is different from large angle excitations, where an increase in external field means an increase in net spin torque, since $\Theta \rightarrow \Theta_{\max }$. 


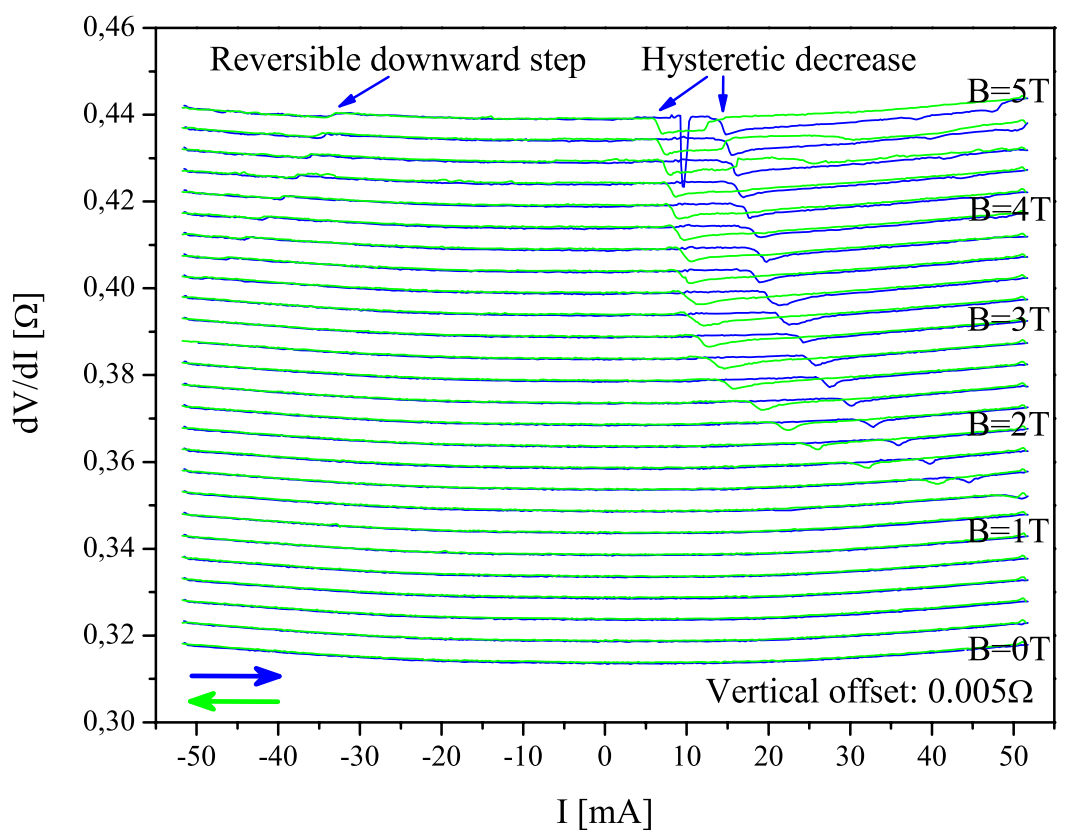

Figure 6.8: Current sweeps for the 6nm thick Co single layer device in the field perpendicular to the plane geometry at fields up to $B=5 T$ and at $T=10 K$.

Therefore large amplitude excitations in an increased applied field require smaller currents in order to be sustained. In terms of these arguments the decrease of $I_{T}$ with increasing magnetic fields for the data presented here might be attributed to prevailing large angle amplitudes.

There is one more parameter, which shows a very distinct field dependence: the absolute value of $\Delta R / R$ rises in higher magnetic fields. This can be deduced from the representative data displayed in figures 6.8 and 6.9 , but it becomes even more obvious when plotting $\Delta R / R$ as a function of $\mu_{0} H$ (cf. figure 6.10). Again, this indicates rather complex excitation patterns and a large variety of precessional orbits, which in return lead to different values for $d V / d I$. The exact identification of all the modes in the data presented within this thesis is not possible. Upon taking into account the intricate feedback mechanism between the single layer magnetization and the spin accumulation in the non-magnetic $\mathrm{Cu}$ the difference to the trilayer geometry (and hence the increased complexity) becomes clear: in the trilayer geometry, the polarizing layer is magnetically 'fixed', thus defining the current polarization. However, in the case of the single layer system, the Co layer itself defines the spin accumulation pattern. When the switching process or precession sets in, the spin accumulation is reduced at the same 


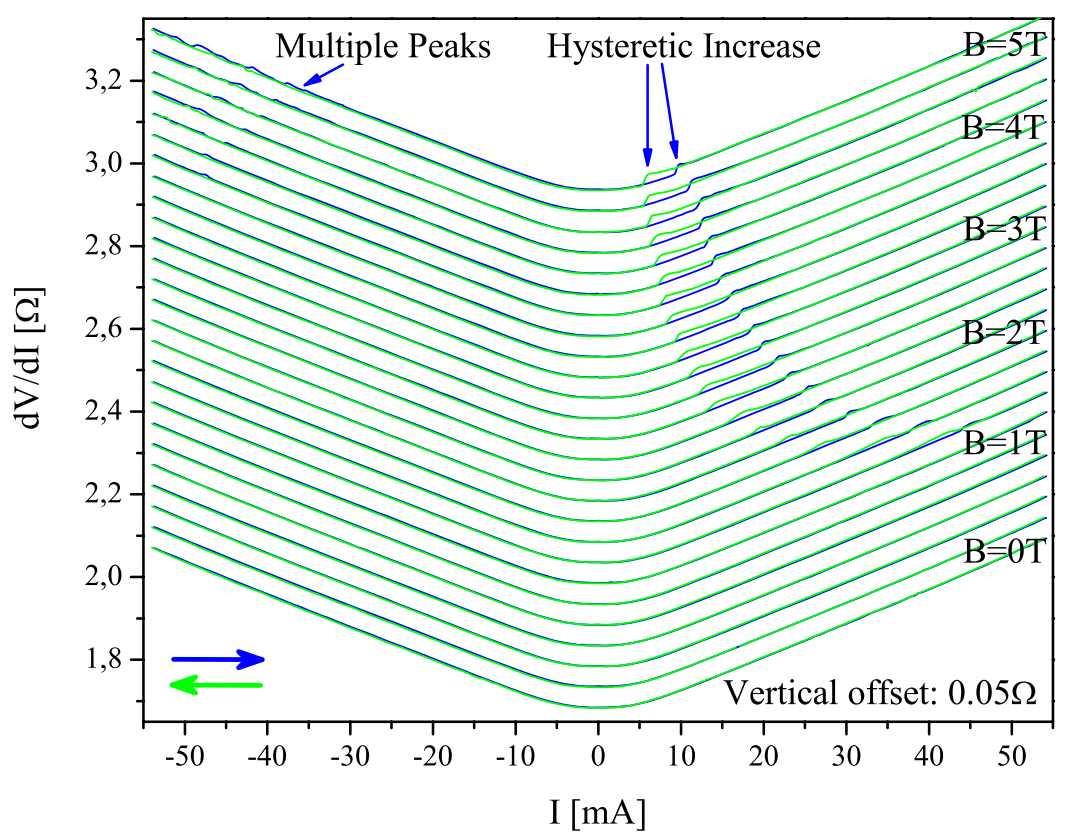

Figure 6.9: Current sweeps for the 8nm thick Co single layer device in the field perpendicular to the plane geometry at fields up to $B=5 T$ and at $T=10 K$.

time. This results in non-linear effects in the excitation pattern. Since the direction of the inhomogeneous magnetization along the $\mathrm{Cu} / \mathrm{Co}$ interface is coupled to the interfacial spin accumulation (and vice versa), an inhomogeneity can be identified. It should be possible to amplify various magnetic eigen modes of the Co film over a large range of currents. Besides, complex and more chaotic excitation patterns have already been found in theoretical models $[33,79,80]$.

\subsubsection{Thickness Dependence of Single Layer Excitations}

Both the theoretical models by Polianski et al. [42] and by Stiles et al. [43] predict an increase of the threshold current $I_{T}$ with increasing layer thicknesses $h$. This becomes comprehensible by taking into account that the spin-transfer torque is essentially generated at the interface, i.e. in the first instance the magnetization in the interfacial regions is set in motion. However, due to the exchange interaction the entire magnetization is affected. Hence, if the ratio of interface to magnetic volume decreases, the spin torque effect becomes less efficient and higher current densities are required.

This tendency is not indicated by the data sets displayed in figure 6.1 

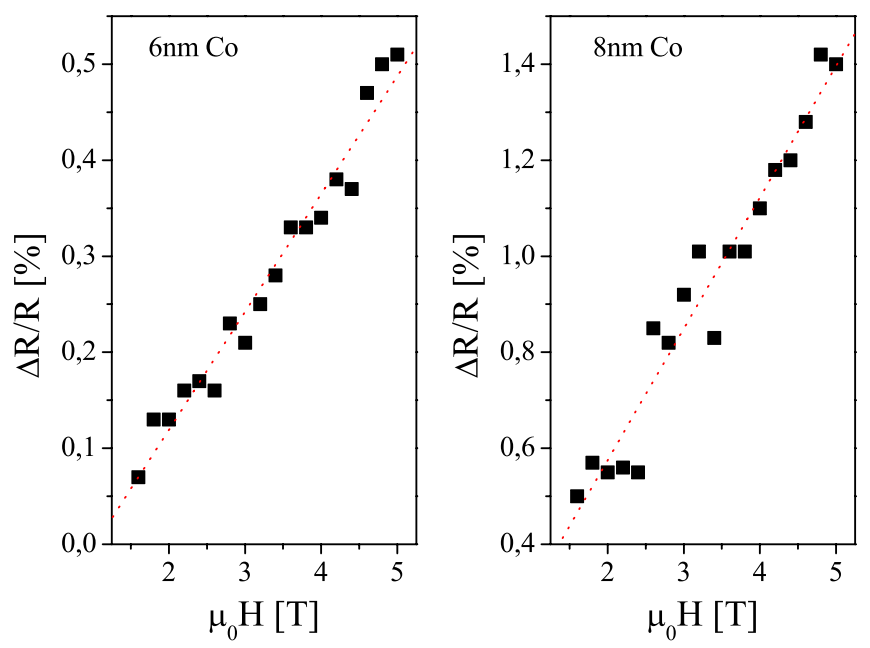

Figure 6.10: $\Delta R / R$ as a function of the external field for the 6nm and the 8nm thick Co layers in the field perpendicular to the plane geometry at $T=10 K$. The dotted red line is just a guide to the eyes.

(with Co thicknesses of $6 \mathrm{~nm}, 8 \mathrm{~nm}, 10 \mathrm{~nm}$ and $12 \mathrm{~nm}$, respectively), which might have multiple causes. First of all, there is a competing effect: due to reference [43] the magnetization might also vary along the direction of current flow - especially for thicker layers where $h<l_{e x}$ (with the exchange length $\left.l_{e x}\right)$. This effect is expected to decrease $I_{T}$. Furthermore, the differences in the total DC resistivities of the four representative nanopillars already imply that caution is advised when directly comparing results from different devices: just like for the trilayer samples, also in the single layer geometry different contact resistances of the electrodes are most likely responsible for the observed resistance deviations for the four devices. This might also alter the concise asymmetry in spin accumulation, which directly influences the magnitude of $I_{T}$ as well.

\subsection{Field Sweeps at Fixed Current Bias in the Field Perpendicular Geometry}

Since for the data shown in the previous sections it is sometimes difficult to distinguish the small features of current-induced excitations from the varying background resistance, some results for field sweeps at a fixed current bias are presented within this section. These measurements do not only eliminate the problem of the varying background, but they also allow for a direct comparison of the current-induced and the field-induced effects. Here representative data for the $8 \mathrm{~nm}$ Co single layer is displayed and subsequently discussed. 


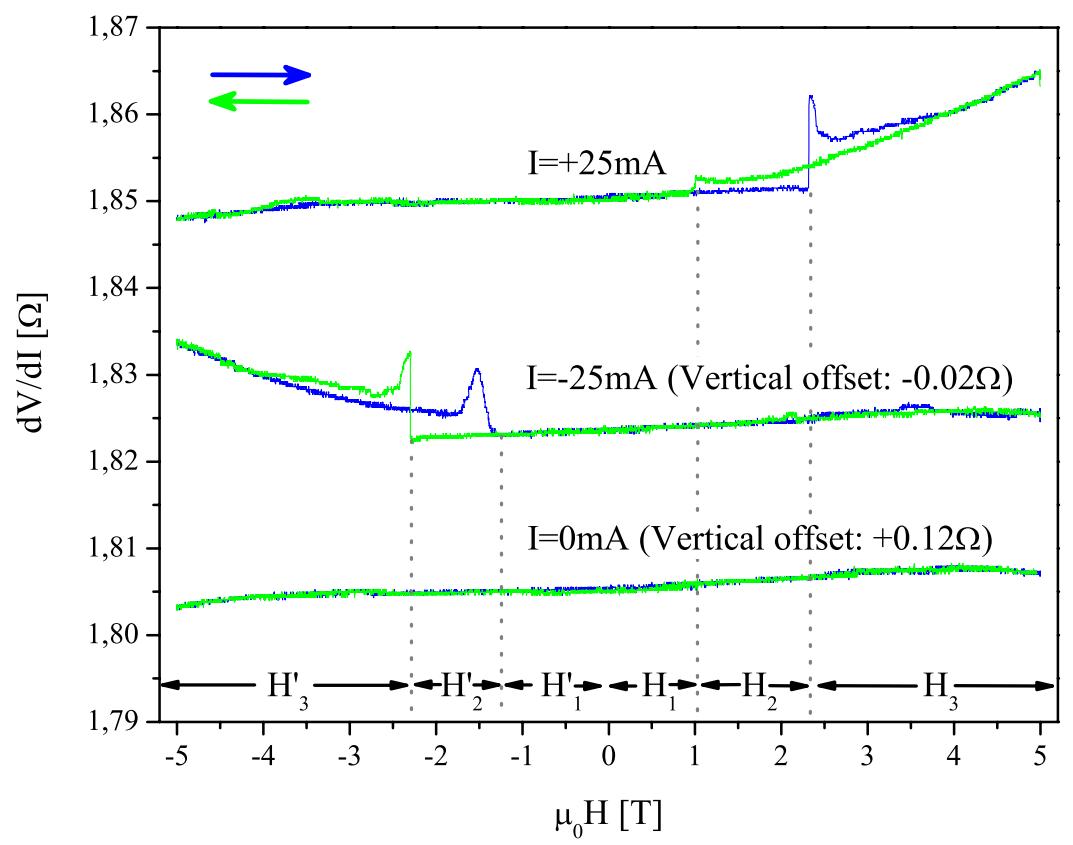

Figure 6.11: Field sweeps at $T=10 \mathrm{~K}$ for the $8 \mathrm{~nm}$ thick single Co layer in the field perpendicular to the plane geometry with a constant current bias of $0 m A,-25 m A$ and $+25 m A$, respectively. Blue lines indicate a sweep direction from $-5 T$ to $+5 T$, whereas the green curves refer to the opposite sweep direction.

Figure 6.11 presents the field sweeps from $+5 \mathrm{~T}$ to $-5 \mathrm{~T}$ (green curves) and the ones for the opposite sweep direction (blue curves) for fixed DC currents of $0 \mathrm{~mA},-25 \mathrm{~mA}$ and $+25 \mathrm{~mA}$, respectively. The loop for $0 \mathrm{~mA}$ bias current shows no abrupt changes in the differential resistance, i.e. it is essentially constant over the entire field range. However, the measurements performed at $+25 \mathrm{~mA}$ yield totally different results: here only the curvature in the regimes of $H_{3}^{\prime}-H_{1}$ is identical to the one detected at $0 \mathrm{~mA}$; no excitations are observed. But the positive high field region of $H_{2}$ is marked by a peak structure followed by a gradual increase of $d V / d I$ (green curve), whereas for the opposite sweep direction (blue line) the excitations are still absent. In the regime of $\mathrm{H}_{3}$ a sharp peak and the subsequent rise of $d V / d I$ can also be identified for the sweeps from negative to positive fields. The total change in differential resistance at $\mu_{0} H=+5 \mathrm{~T}$ amounts to $\Delta R / R=0.91 \%$. The third magnetoresistance loop recorded at $-25 \mathrm{~mA}$ basically reflects the behavior of the curve taken at the opposite current bias of $+25 \mathrm{~mA}$ : here the features detected within the field regime $\mathrm{H}_{2}-\mathrm{H}_{3}$ for positive current bias are found for the inverse field direction within the regions $H_{2}^{\prime}-H_{3}^{\prime}$. The 
width of the hysteresis, the shape of the features, the absolute values of the resistance change as well as the critical field values $H_{C}$ are almost identical to the effects of the current sweep performed at $+25 \mathrm{~mA}$.

In the following section these results are discussed in terms of the influence of the anisotropic magnetoresistance (AMR) effect. Then the correlations of the field sweeps with the current sweeps are addressed in section 6.2.2.

\subsubsection{The Influence of the Classical AMR Effect}

The anisotropic magnetoresistance (AMR) effect refers to the resistance of a material depending on the angle between the direction of the electrical current and the orientation of an external magnetic field. The microscopic origin of this effect is a larger probability of s-d scattering for electrons flowing in the direction of the magnetic field due to the spin-orbit coupling. For a detailed theoretical description the reader is referred to references [81, 82]. As a net result the electrical resistivity usually has its maximum value when the direction of current flow is parallel to the applied magnetic field. However, the AMR effect is a bulk effect, and hence its influence on the $d V / d I$ measurements performed on the nanostructured devices studied within this thesis has to be scrutinized.

The field sweep with zero DC bias current presented in the previous section (figure 6.11) shows no evidence for a dominating influence of the AMR effect: here $d V / d I$ shows no significant change for the magnetization lying in the film plane (low fields) in contrast to the magnetization being orthogonal to the current $I$ (high out of-plane fields $>\mu_{0} M_{S} \approx 1.5 \mathrm{~T}$ ). In fact $d V / d I$ remains almost constant over the entire field range; a maximum gradual change of $0.1 \%$ is detected. This holds also true for the field sweeps at zero DC bias of all the other single layer devices under investigation. Hence, it can be concluded that the AMR effect does not dominate the differential resistance (in particular it does not cause any abrupt changes in $d V / d I$ ), and current-induced features being almost one order of magnitude larger than the AMR effect can be easily identified.

\subsubsection{Current-Induced versus Field-Induced Effects in the Field Perpendicular Geometry}

The absence of any excitations at zero current bias in the field sweeps has already been indicated in the current sweeps (figure 6.9) for the $8 \mathrm{~nm}$ single Co layer. However, the causally related vortex state disappears at sufficiently high fields, so that for a current bias of $+25 \mathrm{~mA}$ the onset of current-induced excitations can be observed at $\mu_{0} H_{C} \approx 1.1 \mathrm{~T}$ (upper curve in figure 6.11 ). In contrast to the current sweeps this starting point is marked by a sharp peak structure. Besides, it is slightly shifted to lower critical field values, which might be attributed to the relatively wide hysteresis that can be identified both in the field and in the current sweeps. Also the subsequent gradual (and almost linear) rise of $\Delta R / R$ with increasing fields is clearly seen in both types of measurements. Altogether, the field sweeps show a satisfactory agreement with the current sweeps, so that the physics behind the 
current-induced resistance change at high fields can be assumed to be very similar to the mechanisms responsible for the field-induced effect at high current bias.

Just like for the trilayer samples both the measurement at $+25 \mathrm{~mA}$ and the one at $-25 \mathrm{~mA}$ are asymmetric around the zero field value, i.e. the features observed for positive magnetic fields are not detected for negative field values and vice versa. Instead of that the reversal of the magnetic field direction is found to correspond to a reversal of the current polarity. Such a particular behavior is observed in the current sweeps of the remaining single layer devices as well. This stands in contrast to the experimental results of other groups $[9,20]$ and to the predictions of the model presented in section 2.8. Again, these observations suggest that even in the high field regime the magnetization within the Co layer cannot be assumed to be homogeneous, making a simple macrospin model deficient. However, the origins of the inhomogeneities cannot be deduced from the transport measurements on the single layers.

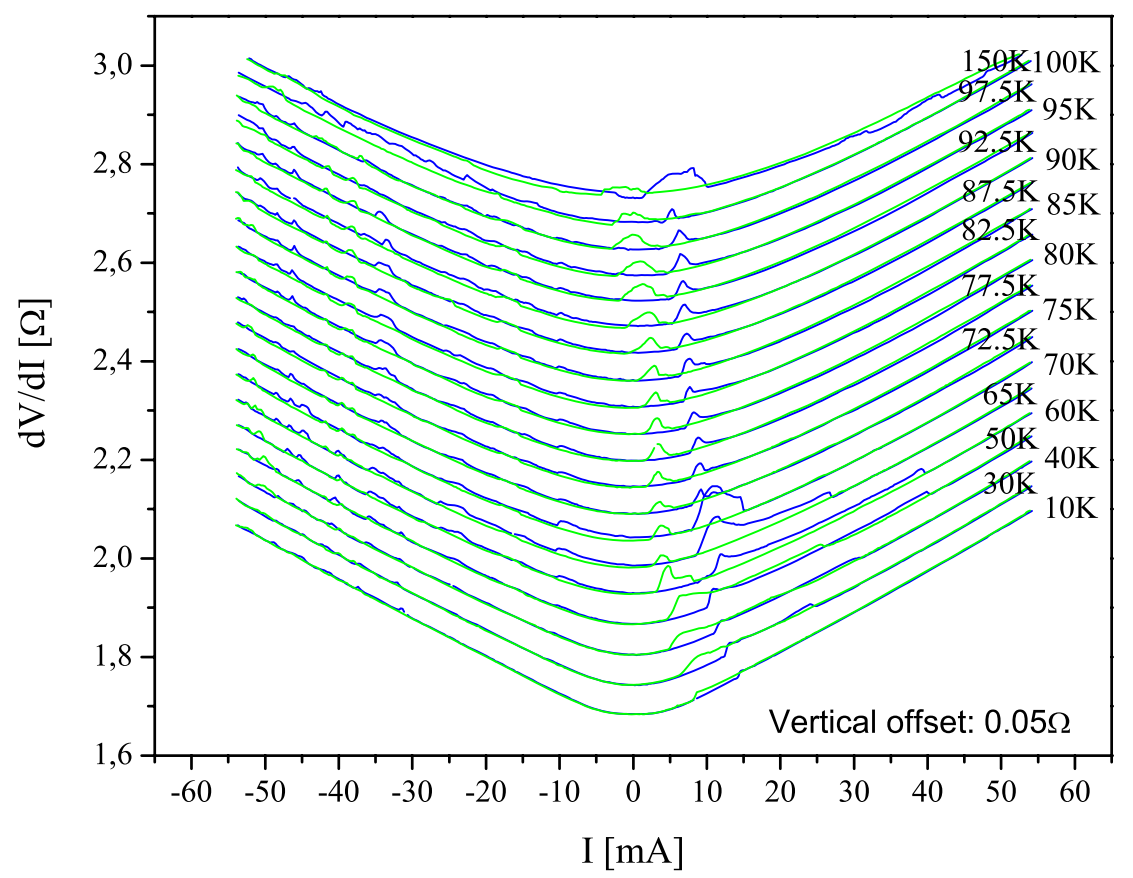

Figure 6.12: Current sweeps at $B=5 T$ and at different temperatures in the field perpendicular to the plane geometry for the 8nm thick Co single layer. 


\subsection{Temperature-Dependent Current Sweeps in the Field Perpendicular Geometry}

In order to investigate the influence of temperature (i.e. thermal activation) on the single layer excitation process, current sweeps at different temperatures have been performed. Here the corresponding data for the $6 \mathrm{~nm}$ thick as well as the $8 \mathrm{~nm}$ thick device in constant external fields of $B=5 \mathrm{~T}$ perpendicular to the sample plane are presented.

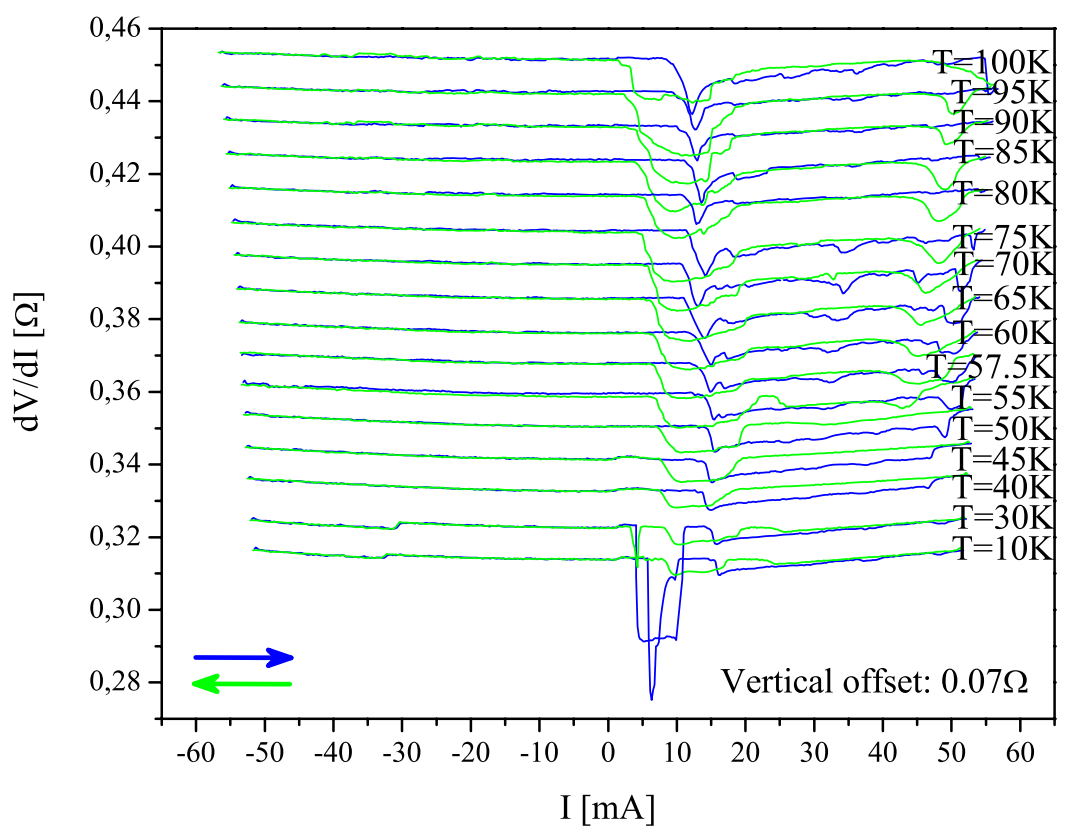

Figure 6.13: Current sweeps at $B=5 T$ and at different temperatures in the field perpendicular to the plane geometry for the 6nm thick Co single layer.

Figure 6.12 shows that the gradual increase observed above the threshold current $I_{T}$ at $T=10 \mathrm{~K}$ slowly evolves to a peak structure with elevated temperatures. Up to $T \approx 50 K$ also the following total resistance change $\Delta R / R$ rises from $1.4 \%$ to approximately $1.8 \%$. For even higher temperature values the distinctive peaks still increase in height up to $T \approx 65 \mathrm{~K}$, while $\Delta R / R$ decreases. Another intriguing tendency is the shift of the excitation threshold $I_{T}$ towards lower values with increasing temperature. At $T \approx$ $87.5 \mathrm{~K}$ the excited state is stable even at $I=0 \mathrm{~mA}$, but only for the sweep directions towards negative current bias (green line); i.e. the curve still shows a strong hysteresis. Moreover, some smaller and quite irregular peaks in $d V / d I$ can be identified at high negative current values for $T \geq 40 \mathrm{~K}$. Also 

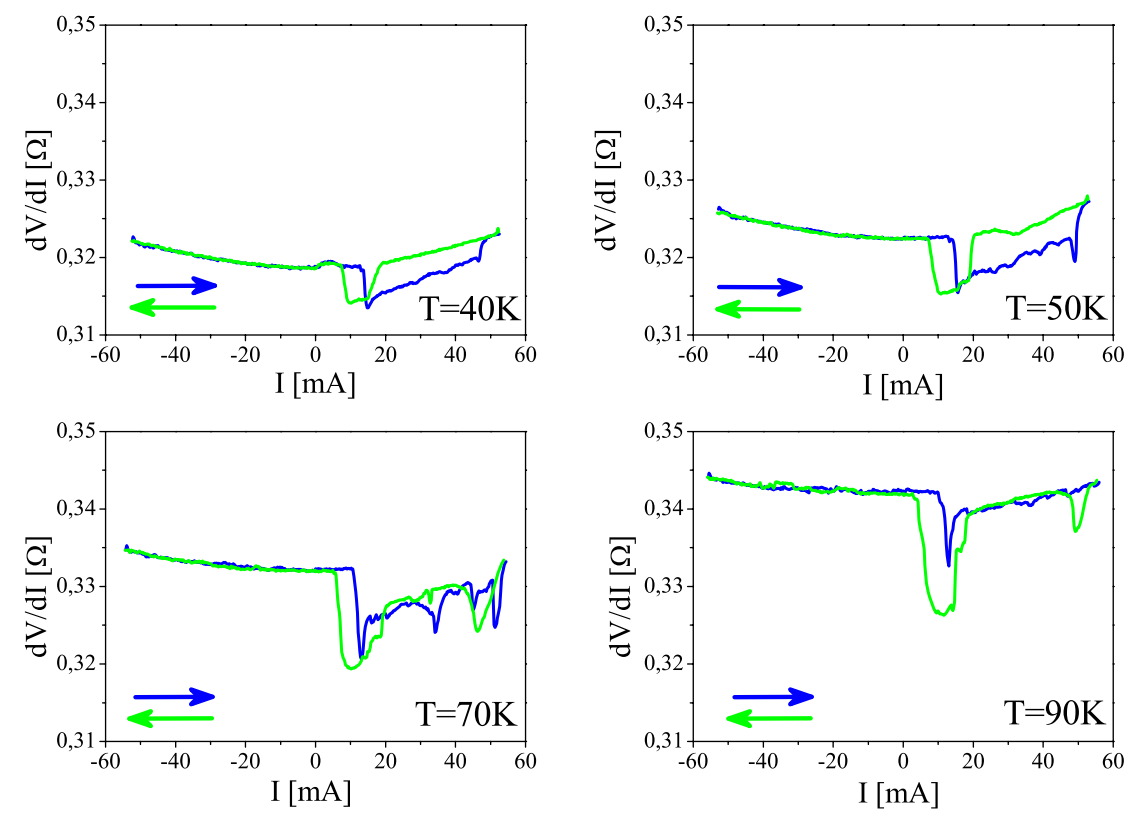

Figure 6.14: Selected current sweeps at $B=5 T$ for temperatures of $T=$ $40 \mathrm{~K}, 50 \mathrm{~K}, 70 \mathrm{~K}$ and $90 \mathrm{~K}$, respectively, in the field perpendicular to the plane geometry for the 6nm thick Co single layer.

these features increase slightly for elevated temperatures.

In figure 6.13 the results of similar measurements as the ones above are displayed for the $6 \mathrm{~nm}$ Co single layer. Here two reductions in $d V / d I$ are dominating the current sweep at $T=10 \mathrm{~K}$ - the decrease being hysteretic for positive current bias and reversible for negative values of $I$. The latter effect vanishes for temperatures above $T \approx 30 \mathrm{~K}$. However, the decrease in the differential resistance at positive current bias becomes much more pronounced at elevated temperatures; figure 6.14 emphasizes this effect by displaying more detailed curves for the temperature values of $40 \mathrm{~K}, 50 \mathrm{~K}, 70 \mathrm{~K}$ and $90 \mathrm{~K}$, respectively. These graphs do not only reveal that the magnitude of $\Delta R / R$ rises at elevated temperatures, but they also show the wide current range of excitations, overlaid by various dips forming a rather broad minimum at temperatures around $T=70 \mathrm{~K}$.

The results are interpreted in the following section.

\subsubsection{The Influence of Elevated Temperatures on Current-Induced Excitations}

The measurements presented in the previous section demonstrate that thermal effects become increasingly important when performing experiments at temperatures higher than the one of liquid He. Finite temperatures can 
make the system fluctuate between different magnetic states, whereupon large fluctuations may even lead to a transition to other stable or metastable configurations. In corresponding theoretical descriptions the thermal effects are either accounted for by a generalized stochastic Landau-Lifshitz equation and its corresponding Fokker-Planck approach [83] or by a barrier crossing model $[84,85]$.

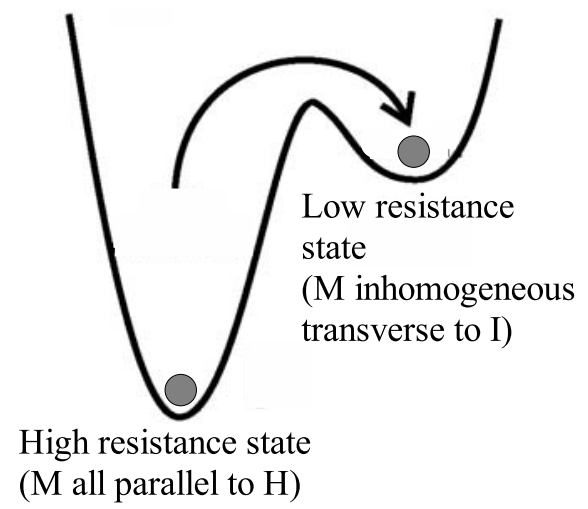

Figure 6.15: Schematic illustration for current-induced hysteretic switching for $B>1.5 T$ and $I>0 m A$ by overcoming a one-dimensional energy barrier (according to the resistance decrease observed in the data set for the 6nm Co layer). Adapted from [87].

The latter model might also intuitively explain the detected decrease of $I_{T}$ with increasing temperatures (cf. figure 6.15): for higher thermal energies the one-dimensional energy barrier between the high resistance state and the magnetic configuration with low resistance can be overcome more easily, thus requiring lower threshold currents for the onset of the switching process. As a consequence the boundaries indicated in figure 6.1 are expected to shift with temperature; i.e. they have a certain amount of indeterminacy. This behavior is confirmed by other experimental studies on magnetic multilayer nanopillars [86] and on trilayer devices [85]. For some sample geometries and in some field regimes, the barrier in both directions is small enough to facilitate thermally driven transitions in both directions. Then two level fluctuations resulting in low frequency noise in $d V / d I$ (as reported by Urazhdin et al. [87]) are generated. However, no direct indications for such telegraph noise are found in the current sweeps of figures 6.12 and 6.13 .

Numerical simulations performed by Russek et al. [88] reveal that changing the temperature also affects the details of the precessional motion: a rise in $T$ introduces stochasticity in the trajectories of the magnetization. Viewed in this context the various dips identified in figure 6.14 at elevated temperatures might be ascribed to diverse precessional orbits causing different changes in the resistivity of the Co layer. 

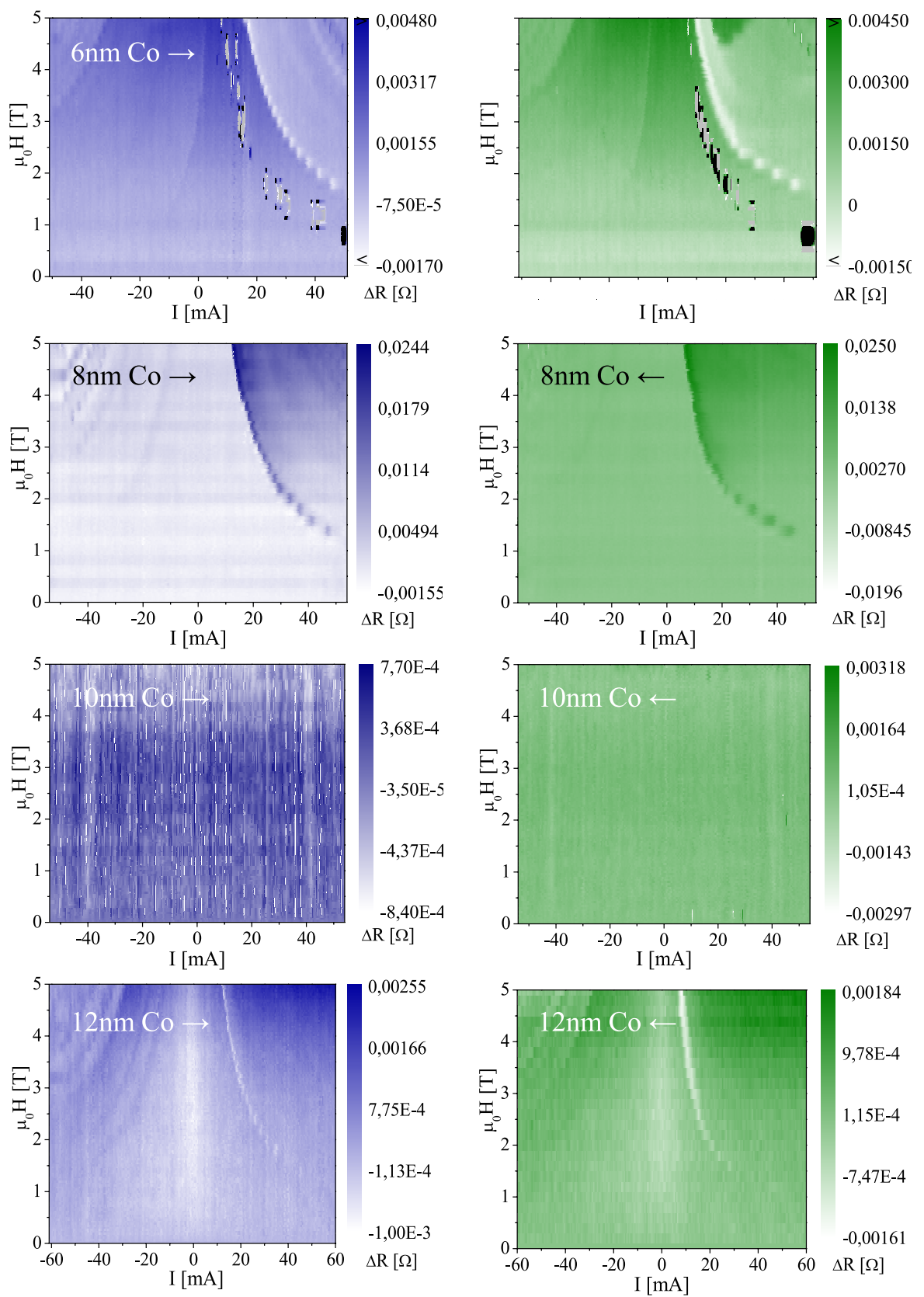

Figure 6.16: Color plots for $d V / d I$ as a function of $I$ and $H$ in the field parallel to the plane geometry. The corresponding Co layer thicknesses and the current sweep directions are indicated in the graphs. Note that for the 6nm Co layer the grey and black colors represent extremely low and extremely high values for $d V / d I$, respectively. 


\subsection{Current Sweeps at Fixed Applied Fields in the Field Parallel Geometry}

The $6 \mathrm{~nm}, 8 \mathrm{~nm}, 10 \mathrm{~nm}$ and $12 \mathrm{~nm}$ thick Co single layers have also been studied in the field parallel to the plane geometry. Also here the applied field dependence of the observed current-induced excitations is best summarized by plotting the current sweeps for both sweep directions separately on color plots (after subtracting the parabolic background). The corresponding results are displayed in figure 6.16.

For all samples except for the 10nm Co device systematic changes in $d V / d I$ with a distinct current and field dependence are detected. Again, the effects vary in shape; some being upward or downward steps, while others are rather similar to peaks and dips. These features are especially pronounced in the data of the $6 \mathrm{~nm}$ Co single layer, where dips with a decrease in the differential resistance of up to $50 \%$ can be identified. Usually these dips are preceded by a peak structure, causing an increase of up to $30 \%$. Such drastic changes could not be included in the color scale of figure 6.16 , so that here dips are simply denoted by a grey tone, and peaks are marked in black. Yet the current sweep depicted in figure 6.17 allows for a detailed representation of the effect. However, the modifications in $d V / d I$ for the

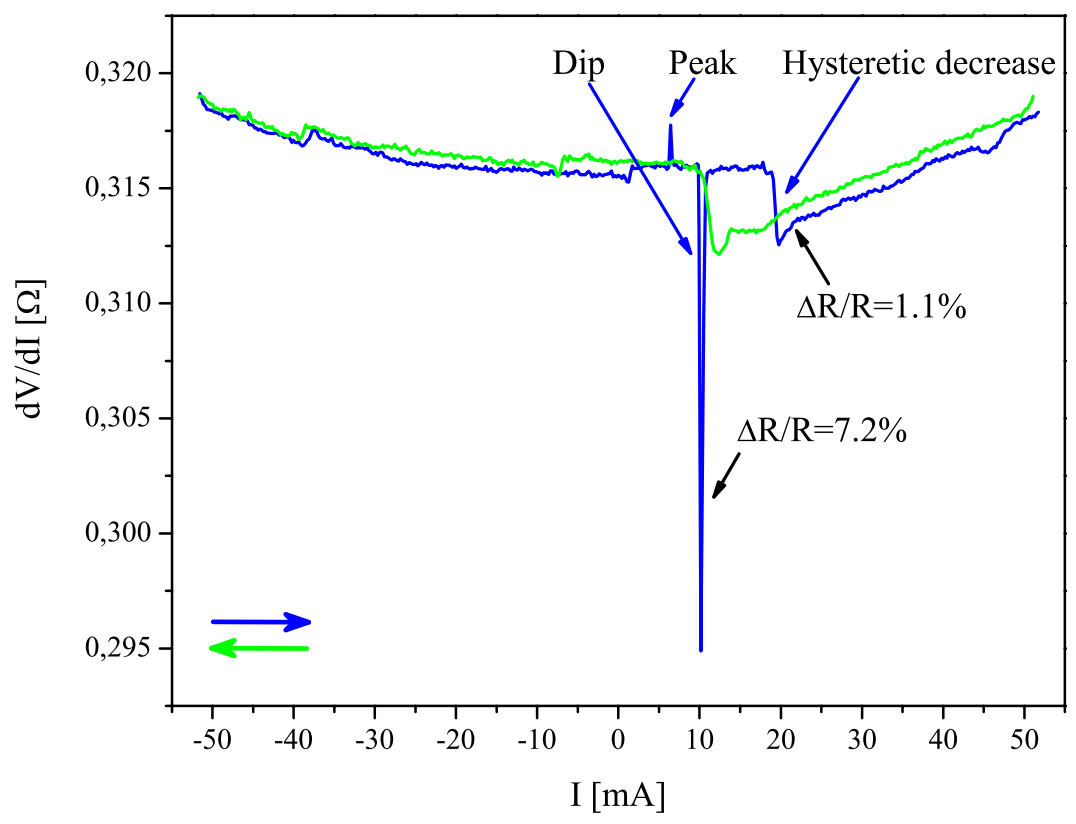

Figure 6.17: Current sweep for the 6nm Co single layer in the field parallel to the plane geometry at $B=4.2 T$ and $T=10 K$. 
other measurements are quite small, yielding maximum values of $1.2 \%$ ( $8 \mathrm{~nm}$ $\mathrm{Co})$ and $0.3 \%(12 \mathrm{~nm} \mathrm{Co})$ for $\Delta R / R$ (calculated at $\mu_{0} H=5 \mathrm{~T}$, because the change in $d V / d I$ is found to increase in higher magnetic fields). The changes in $d V / d I$ are essentially hysteretic when observed for positive current bias, but also reversible steps for the opposite current polarity are detected.

Below a certain field value no excitations in the differential resistance are detectable. The magnitude of this critical field varies: it amounts to approximately $\mu_{0} H=1.5 \mathrm{~T}$ for the $12 \mathrm{~nm}$ and the $8 \mathrm{~nm}$ thick devices, but it is only $0.8 \mathrm{~T}$ for the $6 \mathrm{~nm}$ thick Co single layer. The magnetic field dependence of the threshold and critical currents in higher fields is once more very intriguing, i.e. $I_{T}$ decreases for increasing field values.

The effects described above are discussed in comparison with the field perpendicular to the plane data in the following section.

\subsubsection{Measurements in the Field in Plane versus the Field Perpendicular to the Plane Geometry}

Comparing the color plots of figure 6.1 for the field perpendicular to the plane measurements to the ones of figure 6.16 for the in-plane current sweeps yields no significant differences at first sight: the magnitude of the detected changes in $d V / d I$, the corresponding threshold currents as well as their field dependence seems to be very similar for both magnetic field directions. This is essentially confirmed by the synoptical table 6.1 . Here the most important values, i.e. the magnitude of $\Delta R / R$ at $B=5 \mathrm{~T}$, the critical current $I_{C}$ at $B=5 \mathrm{~T}$ and the critical field $H_{C}$ are summarized.

\begin{tabular}{|c||ccc|ccc|}
\hline \multicolumn{1}{|c||}{} & \multicolumn{3}{c|}{ M perpendicular } & \multicolumn{3}{c|}{ M parallel } \\
\hline \hline$h[\mathrm{~nm}]$ & $\Delta R / R$ & $I_{C}[\mathrm{~mA}]$ & $\mu_{0} H_{C}[\mathrm{~T}]$ & $\Delta R / R$ & $I_{C}[\mathrm{~mA}]$ & $\mu_{0} H_{C}[\mathrm{~T}]$ \\
\hline \hline 6 & 1.0 & 12.7 & 1.4 & 1.1 & 12.9 & 0.8 \\
8 & 1.4 & 9.3 & 1.4 & 1.2 & 11.1 & 1.4 \\
10 & $(0.2)$ & $(-41.2)$ & $(3.6)$ & 0 & - & - \\
12 & 0.5 & 0.2 & 1.4 & 0.3 & 11.5 & 1.6 \\
\hline
\end{tabular}

Table 6.1: Overview of the most important characteristic values for the out-of-plane and the in-plane current sweeps.

Indeed, no significant deviations in $\Delta R / R$ can be identified for the two different magnetic field directions. This suggests that similar changes in the magnetic configuration within the Co are induced by the electrical current - independent of the direction of the external field. However, the excitation thresholds $I_{T}$ and the corresponding critical currents $I_{C}$ are increased for the field in plane geometry. Hence, the efficiency of the spin transfer torque indeed depends strongly on the initial Co magnetization (and the corresponding spin accumulation pattern); especially when the Co volume increases and the ratio of the bulk material to the interface region worsens (see $12 \mathrm{~nm}$ data in table 6.1). The variations in the spin accumulation 
patterns might be related to the different behavior of the vortex structure in out-of-plane and in-plane fields. While the location of the vortex core remains unchanged with increasing perpendicular fields, the surrounding magnetization turns more and more out of plane. But with increasing magnetic in-plane fields, the vortex core moves towards the edge of the Co layer, since the area where the magnetization is parallel to the applied field is enlarged at the cost of the areas with a different magnetization direction.

The very large and abrupt modifications in $d V / d I$ (i.e. the peaks and dips with a resistance change up to $\Delta R / R \approx 50 \%$ ) for the $6 \mathrm{~nm}$ Co layer cannot be ascribed to the interface magnetoresistance effect described above. Having a closer look at the raw data reveals that these peculiar features coincide with abrupt phase shifts for the signal of $d V$. Thus $d V$ and $d I$ are not detected at the same constant phase angle anymore, which results in a huge increase or decrease in $d V / d I$. Since these modifications in the differential resistance also show a systematic field dependence (and they are not detected in the field perpendicular to the plane geometry for the same device), a malfunction of the transport setup and a structural alteration within the sample itself can be excluded. Hence, the drastic changes in $d V / d I$ might be related to the giant magnetoimpedance effect (GMI), which consists in huge changes of the complex impedance of soft magnetic materials upon applying a magnetic field. ${ }^{2}$ However, this point cannot be settled unequivocally.

\subsection{Discussion and Summary for the Single Layer Samples}

The presented measurements on the $6 \mathrm{~nm}, 8 \mathrm{~nm}, 10 \mathrm{~nm}$ and $12 \mathrm{~nm}$ thick Co single layer nanopillars give strong evidence for the existence of currentinduced excitations in spite of the absence of a second ferromagnetic layer. The current sweeps show that also in the single layer geometry the vortex state plays a major role, so that changes in $d V / d I$ can only be observed in sufficient external magnetic fields: then the more homogeneous magnetization within the Co leads to a significant build-up of spin accumulation at the $\mathrm{Cu} / \mathrm{Co}$ interfaces. Thus, at sufficient current densities the spin-transfer torque leads to modifications in the differential resistance in the shape of peaks, dips and downward as well as upward steps.

It is shown that a decrease in $d V / d I$ can generally be attributed to a transverse inhomogeneity in the magnetization pattern, whereas an increase in single layer resistance seems to be closely related to an inhomogeneous magnetization configuration along the direction of current flow. However, the anomalous field behavior of the threshold current (i.e. the decrease of $I_{T}$ with increasing magnetic fields) and the increase of the total resistance change $\Delta R / R$ with $H$ reveal that the true magnetization configuration within the Co is most likely much more complex than the models suggest. This is also rooted in the sophisticated feedback mechanism between the

\footnotetext{
${ }^{2}$ For reviews on the GMI effect the reader is referred to references [90, 91].
} 
single layer magnetization and the spin accumulation in the non-magnetic $\mathrm{Cu}$ : the magnetization within the Co layer itself defines the spin accumulation pattern, so that as soon as precession sets in, the spin accumulation is decreased. In this way rather complicated excitation patterns are produced, which is reflected in the manifold modifications in $d V / d I$. With increasing temperatures these excitations are found to shift to lower threshold currents $I_{T}$; i.e. thermal activation promotes transitions to other stable or metastable states. However, the identification of the excited modes in the transport data is not possible - even the distinction between static and dynamic states turns out to be challenging.

For this purpose alternative measurement techniques are necessary. Just like for the trilayer samples, also for the single layer nanopillars timeresolved XMCD microscopy or frequency-domain measurements using a spectrum analyzer might give additional valuable insight into the magnetization dynamics of the Co layers.

Furthermore, the comparability of samples with different thicknesses has to be improved. For this purpose a shadow mask system with the ability to increase the number of nanopillar contacts per sample from six to thirty has recently been implemented in the preparation process. Hence it will be possible to vary the Co layer thickness by using a wedge shaped thickness variation on one sample for exactly equal growth conditions. This comprises both comparable interface roughnesses and preferably similar contact resistances. 


\section{CHAPTER 7}

\section{Concluding Remarks}

The aim of this thesis has been to develop a lithographic preparation process and a measurement setup in order to shed light on spin transfer induced excitations in all-metal ferromagnetic nanostructures. The following section 7.1 summarizes the most important findings, while in section 7.2 some suggestions for further fundamental experiments are presented.

\subsection{Summary}

A considerable part of this thesis work has been devoted to the development of a feasible geometry for a ferromagnetic nanodevice, which has a small cross-sectional area and allows for a magnetoresistive readout of the magnetic state. The result is a simplified one step process, in which circular holes with a diameter below $100 \mathrm{~nm}$ are patterned into a conventional PMMA resist by means of e-beam lithography. Since PMMA is a good electric insulator and a smooth buffer layer for subsequent film growth, it is directly used as a template for the evaporation of the ferromagnetic layer stack. The corresponding bottom and top electrodes are defined by evaporation through a mechanical shadow mask. Thus the magnetic nanopillars can be fabricated without any additional lift-off, ion milling or reactive ion etching steps.

SEM investigations on the cross-sections of the finished nanodevices reveal that the preparation process described above yields accurate pillar structures with relatively steep sidewalls. The corresponding TEM and EDX analyses indicate a stack of well-defined layers unsuggestive of any parallel conductance channels such as shunting effects along the edges of the nanopillars or even parasitic shorts between the top and the bottom electrodes. Besides, it is confirmed that the nanopillars are approximately 80nm in diameter. 
Because of these small dimensions the magnetic characterization of the ground state of the samples turned out to be very challenging: since the maximum resolution accomplishable in the utilized XMCD-PEEM tool is approximately 20nm, these experiments do not allow for an unambiguous identification of either a vortex or a single domain (i.e. a totally homogeneously magnetized) state. Hence micromagnetic simulations are performed in order to determine the magnetic configuration with the lowest total energy as a function of the nanopillar thickness. According to these simulations, in the absence of an external field the vortex state is stable for thicknesses above $9.5 \mathrm{~nm}$ in circular structures with a diameter of $80 \mathrm{~nm}$. For smaller thicknesses a single domain state is discovered to be energetically favorable.

For the transport characterization of the samples an experimental set-up, which allows for the measurement of the differential resistance $d V / d I$ as a function of the DC current in a four-point measurement geometry, has been designed. This transport setup provides a resolution better than $0.5 \mathrm{~m} \Omega$.

For sufficient DC current densities in the region of $10^{8} \mathrm{~A} / \mathrm{cm}^{2}$ and high magnetic fields the transport data for the $3 \mathrm{nmCo} / 10 \mathrm{nmCu} / 12 \mathrm{nmCo}$ GMR trilayer nanopillars show changes in $d V / d I$ (up to $\Delta R / R=2.7 \%$ ), which are asymmetric in current: this asymmetry is the signature of the spin-transfer torque, indicating that the effect is induced by the spin polarization of the current rather than by its Maxwell-Oersted field. The shapes of the modifications in the differential resistance are manifold. Peak structures are ascribed to uniform precessional states, while the hysteretic changes in $d V / d I$ result from quasi static modifications in the magnetization configuration of the thin Co layer. In the most simple semi-classical spin torque model the changes can be attributed to current-induced switching processes of the thin Co layer between two different single domain states: the parallel (low resistance) and the antiparallel (high resistance) state with respect to the thicker and hence magnetically fixed Co layer. However, the absence of excitations for field values below the demagnetization field of $\mu_{0} M_{S} \approx 1.5 \mathrm{~T}$ indicates that the magnetization configuration within both Co layers is much more complex and cannot be captured by a simple macrospin model. This is supported by the existence of further low resistance states, which are ascribed to the nucleation of vortices due to the influence of the Maxwell-Oersted field. Also the field sweeps at fixed current bias reveal that the actual magnetization configuration within the Co layers is inhomogeneous and not fully aligned with the external field even for higher field values (asymmetry for high current densities, no saturation for higher external fields at zero bias current). Nonetheless these magnetoresistance loops show a good correspondence with the current sweeps. From the combination of these two measurement techniques phase diagrams, which display some possible magnetization configurations including the phase boundaries for the threshold and critical currents in the 'current bias - magnetic field' plane, are constructed.

The transport measurements on the Co single layer nanopillars show that current-induced excitations can even evolve in the absence of a second fer- 
romagnetic layer, which emphasizes the spin filtering properties of a single $\mathrm{Cu} / \mathrm{Co}$ interface. Just like for the trilayer samples a large variety of modifications in $d V / d I$ are observed - but only for large field values. This is once more attributed to the existence of a vortex state, which avoids the development of a sufficient spin accumulation in lower magnetic fields. Within a qualitative model a decrease in $d V / d I$ is attributed to a transverse inhomogeneity in the magnetization pattern, whereas an increase in single layer resistance results from an inhomogeneous magnetization configuration longitudinal to the current direction. However, the anomalous field behavior of the threshold current and the increase of the total resistance change $\Delta R / R$ with $H$ reveal that the true magnetization configuration within the Co is again most likely much more complicated than the models suggest. This indicates the complex feedback mechanism between the Co magnetization and the spin accumulation in the non-magnetic $\mathrm{Cu}$ : the magnetization within the Co layer itself defines the spin accumulation pattern, so that as soon as precession sets in, the spin accumulation is decreased. As a result also the net spin torque effect is reduced, which might in return lead to a change of the precessional orbit. However, since the transport measurements performed here provide only indirect evidence on the nature of excitations, the different modes cannot be unambiguously identified.

\subsection{Outlook}

The results presented above are beneficial for a qualitative comparison to existing theoretical spin-transfer models. However, for a more quantitative test the variability from sample to sample has to be significantly decreased. Since sometimes the results from nominally identical devices have been found to vary substantially in detail, the preparation process has to be refined. One possibility to achieve this is to increase the number of nanopillars per sample, so that the nanocontacts are grown under exactly identical conditions. A step towards this direction has recently been made by fabricating a shadow mask system which allows for the simultaneous preparation of thirty nanopillars per sample. In combination with the movable shutter of the UHV chamber, this system even allows for the preparation of wedge shaped samples - i.e. the thicknesses of the ferromagnetic or the non-magnetic spacer layers can be varied systematically.

A further limitation was the informative value of the DC transport measurements: this technique gives no details on the spatial and the temporal evolution of the magnetization within the Co layers. But then this is the most interesting part of the current-induced switching effect, because most likely only its direct observation will lead to a much better understanding of the underlying processes on the nanometer length scale and on a time scale extending into the picosecond range.

A technique which grants access to the time domain is the electrical measurement of high-frequency magnetization dynamics generated in the nanostructures. Here the voltage oscillations produced by the changing relative 
angle between the magnetic moments of the two ferromagnetic layers are recorded with a heterodyne spectrometer circuit. More technical details on this method can be found in reference [89]. However, once more this technique provides only indirect spatial information: by comparing the excitations detected in the microwave power spectra to single-domain simulations, some excited mode structures can be identified.

Direct detection of the spin transfer places high demands on the experimental equipment, because the excited ferromagnetic volume is extremely small and besides, it is buried under a thin film stack. These challenges can be met by utilizing the scanning transmission x-ray microscopy (STXM) technique, which has been pioneered be the Stöhr group at the Advanced Light Source [76, 92]. Upon depositing the sample on a SiN membrane, which is largely transparent to x-rays, a single ferromagnetic layer within the nanopillar structure can be investigated with a spatial resolution down to a few nanometers. Time resolution can then be added by synchronizing a Ti:sapphire laser with the pulsed synchrotron light. The laser can activate an optical switch, and an ultrashort current pulse with a duration of less than $15 p$ s is emitted [93]. In this connection an adjustable optical delay determines the delay time between the pulse for the generation of the current and the x-ray bunch. Thus the x-ray microscope stroboscopically measures the magnetic response of the ferromagnetic layer to the injected current in a pump-probe fashion.

However, a comparison to theoretical models is desirable. Since this is rather difficult for nanodevices with an inhomogeneous magnetization like the ones presented in this work, a slight modification of the sample geometry might also be helpful. The magnetooptical studies on Supermalloy nanomagnet arrays performed by Cowburn et al. [94] reveal that the introduction of shape anisotropy can result in single domain behavior for sufficiently small structures. Moreover, single domain states and the corresponding transitions could improve the device performance. Hence, investigations on nanopillars with an elliptical cross-sectional area might be a matter of particular interest. Alternatively, the homogeneity of the magnetization within the polarizing layer could be improved by adding a (synthetic) antiferromagnetic layer, which strongly pins the ferromagnetic layers in an antiparallel configuration. 


\section{Bibliography}

[1] L. Berger, Phys. Rev. B 54, 9353 (1996)

[2] J. C. Slonczewski, J. Magn. Magn. Mater. 159, L1 (1996)

[3] M. Tsoi, A. G. M. Jansen, J. Bass, W.-C. Chiang, M. Seck, V. Tsoi, P. Wyder, Phys. Rev. Lett. 80, 4281 (1998)

[4] E. B. Myers, D. C. Ralph, J. A. Katine, R. N. Louie, R. A. Buhrman, Science 285, 867 (1999)

[5] J. E. Wegrowe, D. Kelly, Y. Jaccard, P. Guittienne, J. P. Ansermet, Europhys. Lett. 45, 626 (1999)

[6] J. Z. Sun, J. Magn. Magn. Mater. 202, 157 (1999)

[7] Y. Ji, C. L. Chien, M. D. Stiles, Phys. Rev. Lett. 90, 106601 (2003)

[8] T. Y. Chen, Y. Ji, C. L. Chien, M. D. Stiles, Phys. Rev. Lett. 93, 026601 (2004)

[9] B. Özyilmaz, A. D. Kent, J. Z. Sun, M. J. Rooks, R. H. Koch, Phys. Rev. Lett. 93, 176604 (2004)

[10] F. J. Himpsel, D. E. Eastman, Phys. Rev. B 21, 3207 (1980)

[11] G. J. Mankey, R. F. Willis, F. J. Himpsel, Phys. Rev. B 48, 10284 (1993)

[12] H. Ibach, H. Lüth, Solid State Physics, Springer, Berlin (1993) 
[13] R. E. Camley, T. S. Rahman, D. Mills, Phys. Rev. B 27, 261 (1983)

[14] N. F. Mott, Proc. Royal Soc. 156, 368 (1936)

[15] E. C. Stoner, Proc. Royal Soc. A165, 372 (1938)

[16] R. Knorren, K. H. Bennemann, R. Burgermeister, M. Aeschlimann, Phys. Rev. B 61, 9427 (2000)

[17] F. J. Jedema, A. T. Filip, B. J. van Wees, Nature 410, 345 (2001)

[18] B. A. Gurney et al., Phys. Rev. Lett. 71, 4023 (1993)

[19] N. W. Ashcroft, N. D. Mermin, Solid State Physics, Saunders, Orlando 1976

[20] B. Özyilmaz, PhD Thesis, New York University (2005)

[21] P. C. van Son, H. van Kempen, P. Wyder, Phys. Rev. Lett. 58, 2271 (1987)

[22] T. Valet, A. Fert, Phys. Rev. B 48, 7099 (1993)

[23] A. Shpiro, PhD Thesis, New York University (2004)

[24] M. A. M. Gijs, G. E. W. Bauer, Adv. Phys. 46, 285 (1997)

[25] A. Barthelemy, A. Fert, F. Petroff, Handbook of Magnetic Materials, Volume 12, Elsevier Amsterdam (1999)

[26] J. Bass, W. P. Pratt, J. Magn. Magn. Mater. 200, 274 (1999)

[27] L. Piraux, S. Dubois, A. Fert, L. Belliard, European J. Phys. B 4, 413 (1998)

[28] A. Fert, J.-M. George, H. Jaffres, G. Faini, J. Phys. D: Appl. Phys. 35, 2443 (2002)

[29] G. Binash, P. Grünberg, F. Saurenbach, W. Zinn, Phys. Rev. B 39, 4828 (1989)

[30] M. N. Baibich, J. M. Broto, A. Fert, F. Nguyen Van Dau, F. Petroff, P. Etienne, G. Greuzet, A. Friederich, J. Chazelas, Phys. Rev. Lett. 61, 2472 (1988)

[31] J. F. Gregg, I. Petej, E. Jouguelet, C. Dennis, J. Phys. D: Appl. Phys. 35, R121 (2002)

[32] J. A. Katine, F. J. Albert, R. A. Buhrman, E. B. Myers, D. C. Ralph, Phys. Rev. Lett. 84, 3149 (2000) 
[33] M. D. Stiles, J. Miltat, Spin Transfer Torque and Dynamics in Spin Dynamics in Confined Magnetic Structures III, edited by B. Hillebrands and A. Thiaville, Springer Berlin (2006)

[34] M. D. Stiles, A. Zangwill, Phys. Rev. B 66, 014407 (2002)

[35] D. E. Buergler, Advanced Magnetic Switching Concepts, 36. IFF-Ferienkurs, Magnetism goes Nano, Jülich (2005)

[36] J. Bass, S. Urazhdin, N. O. Birge, W. P. Pratt Jr., Phys. Stat. Sol. 201, 1379 (2004)

[37] J. Miltat, G. Albuquerque, A. Thiaville, An Introduction to Micromagnetics in the Dynamic Regime, in Spin Dynamics in Confined Magnetic Structures I, Springer Berlin (2002)

[38] S. Zhang, P. M. Levy, A. Fert, Phys. Lett. 88, 236601 (2002)

[39] A. Shpiro, P. M. Levy, S. Zhang, Phys. Rev. B 67, 104430 (2003)

[40] J. C. Slonczewski, J. Magn. Magn. Mater. 247, 324 (2002)

[41] S. Mangin, D. Ravelosona, J. A. Katine, M. J. Carey, B. D. Terris, E. E. Fullerton, Nature Materials 5, 210 (2006)

[42] M. L. Polianski, P. W. Brouwer, Phys. Rev. Lett. 92, $026602(2004)$

[43] M. D. Stiles, J. Xiao, A. Zangwill, Phys. Rev. B 69, 054408 (2004)

[44] A. Brataas, Y. Tserkovnyak, G. Bauer, Phys. Rev. B 73, 014408 (2006)

[45] S. Adam, M. L. Polianski, P. W. Brouwer, Phys. Rev. B 73, 024425 (2006)

[46] M. A. McCord, M. J. Rooks, Handbook of Microlithography, Micromachining and Microfabrication, Volume 1, SPIE-The International Society for Optical Engineering, Bellingham, WA (1997)

[47] G. Eilers, Diploma Thesis, Göttingen University (2004)

[48] J. Z. Sun, D. J. Monsma, D. W. Abraham, M. J. Rooks, R. H. Koch, J. Appl. Phys. 81, 2202 (2002) 
[49] P. Ebert, K. Szot, Scanning Probe Microscopy, 32. IFFFerienkurs, Neue Materialien für die Informationstech$n i k$, Jülich (1997)

[50] J. Ackermann, Handbuch für die Rasterelektronenmikroskope SUPRA(VP) und ULTRA, Carl Zeiss NTS $\mathrm{GmbH}, 2004$

[51] G. Brewer, ed., Electron-Beam Technology in Microelectronic Fabrication, Academic Press (1980)

[52] Product data sheet for Nova 600 NanoLab by FEI

[53] Product brochure for the PPMS, Quantum Design

[54] C. M. Schneider, G. Schönhense, Rep. Prog. Phys. 65, R1785 (2002)

[55] W. Kuch, Magnetic Imaging, in Magnetism: A Synchrotron Approach, Springer Berlin (2006)

[56] J. Stöhr, J. Magn. Magn. Mater. 200, 470 (1999)

[57] A. Rogalev, F. Wilhelm, N. Jaouen, J. Goulon, J.-P. Kappler, X-ray Magnetic Circular Dichroism: Historical Perspective and Recent Highlights, in Magnetism: A Synchrotron Approach, Springer Berlin (2006)

[58] H. Kano, K. Kagawa, A. Suzuki, A. Okabe, K. Hayashi, K. Asoa, Appl. Phys. Lett. 63, 2839 (1993)

[59] M. Suzuki, Y. Taga, Phys. Rev. B 52, 361 (1995)

[60] E. Y. Tsymbal, D. G. Pettifor, Perspectives of Giant Magnetoresistance, in Solid State Physics 56, Academic Press (2001)

[61] H. Hoffmann, Festkörperprobleme, Volume XXII, Vieweg, Braunschweig (1982)

[62] The Object Oriented Micro Magnetic Framework (OOMMF) project at the NIST, available at http://math.nist.gov/oommf/

[63] M. J. Donahue, D. G. Porter, Physica B 343, 177 (2004)

[64] M. J. Donahue, R. D. McMichael, Physica B 233, 272 (1997)

[65] W. H. Rippard, M. R. Pufall, T. J. Silva, Appl. Phys. Lett. 82, 1260 (2003) 
[66] B. Özyilmaz, A. D. Kent, D. Monsma, J. Z. Sun, M. J. Rooks, R. H. Koch, Phys. Rev. Lett. 91, 0672031 (2003)

[67] W. Chen, M. J. Rooks, N. Ruiz, J. Z. Sun, A. D. Kent, Phys. Rev. B 74, 144408 (2006)

[68] J. Sun, Nature 425, 359 (2003)

[69] J. Grollier, V. Cros, A. Hamzic, J. M. George, H. Jaffres, A. Fert, G. Faini, J. Ben Youssef, H. Legall, Appl. Phys. Lett. 78, 3663 (2001)

[70] S. I. Kiselev, J. C. Sankey, I. N. Krivorotov, N. C. Emley, M. Rinkoski, C. Perez, R. A. Buhrman, D. C. Ralph, Phys. Rev. Lett. 93, 0366011 (2004)

[71] J. Z. Sun, D. J. Monsma, T. S. Kuan, M. J. Rooks, D. W. Abraham, B. Özyilmaz, A. D. Kent, R. H. Koch, J. Appl. Phys. 93, 6859 (2003)

[72] F. J. Albert, J. A. Katine, R. A. Buhrman, D. C. Ralph, Appl. Phys. Lett. 77, 3809 (2000)

[73] S. Urazhdin, C. L. Chien, K. Y. Guslienko, L. Novozhilova, Phys. Rev. B 73, 054416 (2006)

[74] B. Özyilmaz, A. D. Kent, M. J. Rooks, J. Z. Sun, Phys. Rev. B 71, 140403(R) (2005)

[75] I. N. Krivorotov, N. C. Emley, J. C. Sankey, S. I. Kiselev, D. C. Ralph, R. A. Buhrman, Science 307, 228 (2005)

[76] Y. Acremann, J. P. Strachan, V. Chembrolu, S. D. Andrews, T. Tyliszczak, J. A. Katine, M. J. Carey, B. M. Clemens, H. C. Siegmann, J. Stöhr, Phys. Rev. Lett. 96, 217202 (2006)

[77] R. D. McMichael, M. D. Stiles, J. Appl. Phys. 97, 10J901 (2005)

[78] J. B. van Hoof, K. M. Schep, A. Brataas, G. E. W. Bauer, P. J. Kelly, Phys. Rev. B 59, 138 (1999)

[79] D. V. Berkov, N. Gorn, Phys. Rev. B 71, 052403 (2005)

[80] Z. Li, Y. Charles Li, and S. Zhang, Phys. Rev. B 74, 054417 (2006)

[81] H. Ebert, A. Vernes, J. Banhart, Phys. Rev. B 54, 8479 (1996) 
[82] I. A. Campbell, A. Fert, O. Jaoul, J. Phys. C 1, S95 (1970)

[83] Z. Li, S. Zhang, Phys. Rev. B 69, 134416 (2004)

[84] R. H. Koch, J. A. Katine, J. Z. Sun, Phys. Rev. Lett. 92, $088302(2004)$

[85] E. B. Myers, F. J. Albert, J. C. Sankey, E. Bonet, R. A. Buhrman, D. C. Ralph, Phys. Rev. Lett. 89, 196801 (2002)

[86] M. Tsoi, J. Z. Sun, M. J. Rooks, R. H. Koch, S. S. P. Parkin, Phys. Rev. B 69, 100406 (2004)

[87] S. Urazhdin, N. O. Birge, W. P. Pratt Jr., J. Bass, Phys. Rev. Lett. 91, 146803 (2003)

[88] S. E. Russek, S. Kaka, W. H. Rippard, M. R. Pufall, T. J. Silva, Phys. Rev. B 71, 104425 (2005)

[89] S. I. Kiselev, J. C. Sankey, I. N. Krivorotov, N. C. Emley, R. J. Schoelkopf, R. A. Buhrman, D. C. Ralph, Nature 425, 380 (2003)

[90] M. Knobel, K. R. Pirota, J. Magn. Magn. Mater. 242245, 33 (2002)

[91] M. Vazquez, J. Magn. Magn. Mater. 226-230, 693 (2001)

[92] S.-B. Choe, Y. Acremann, A. Scholl, A. Bauer, A. Doran, J. Stöhr, H. A. Padmore, Science 304, 420 (2004)

[93] S. Bretschneider, Diploma Thesis, Göttingen University (2004)

[94] R. P. Cowburn, D. K. Koltsov, A. O. Adeyeye, M. E. Welland, D. M. Tricker, Phys. Rev. Lett. 83, 1042 (1999) 


\section{Acknowledgements}

I would like to express my gratitude to all of you who have contributed to this thesis in one way or another. Special thanks goes to:

Prof. Dr. Markus Münzenberg for being my supervisor for this work. Your door has always been open whenever I needed your input or just some cheering-up. A sincere thanks for your sound advice and patience.

PD Dr. Christian Jooß for being the second referee for this thesis.

Gerrit Eilers for lots of advice at all times. I have benefit a whole lot from your experimental skills - especially when agonizing over all these horrible electrical circuits or intractable PC programs. I truly appreciate your support, and I am not really sure where this thesis would have gone without it.

Dr. Marija Djordjevic Kaufmann for the fruitful discussions - whether they were about physics or so many other important issues in life. Thank you for all the encouragement and for your great friendship over the past three years.

Malte Scherff for helping out with long and nerve-wracking sample preparation hours towards the end of this thesis.

Much respect to my former and present officemates and all other student colleagues at Physics IV for putting up with me throughout the past years, not forgetting all the people at Physics I. Thank you all for providing such a stimulating and fun environment!

Markus Esseling and Uta Bete from Physics I for the constant assistance in trouble-shooting technical problems at this bewildering e-beam 
lithography tool.

Dr. Mathias Kläui and his PhD students from the University of Konstanz for performing the XMCD-PEEM experiments at the Swiss Light Source - a true scientific challenge.

Volker Radisch for the preparation of the TEM lamellas with the FIB. Thank you for your persistency.

PD Dr. Michael Seibt and Dr. Tore Niermann, who carried out the TEM experiments.

The very reliable staff-crew of Physics IV, especially Antje SpliethoffLaiser, Bernhard Spicher and Andreas Juretzko.

Saskia Bellem, Marija Djordjevic Kaufmann, Gerrit Eilers and Georg Müller for proof-reading the manuscript.

My friends outside the physics world for providing the necessary distraction from $\mathrm{PhD}$-associated struggles and tribulations.

My parents, my brother and Tobias for being a constant source of support - emotional and moral. 



$\begin{array}{ll}\text { Name: } & \text { Anne Parge } \\ \text { Geburtsdatum: } & 17.06 .1979 \\ \text { Geburtsort: } & \text { Kiel } \\ \text { Staatsangehörigkeit: } & \text { deutsch }\end{array}$

SCHULAUSBILDUNG

06/1998 Humboldt Schule Kiel

Abitur

08/1995- SAS School, St. Andrews, TN, USA

06/1996 Auslandsschuljahr

HOCHSCHULAUSBILDUNG

$10 / 2003$

Georg-August-Universität Göttingen

Beginn der Promotion

Georg-Christoph-Lichtenberg-Stipendiatin

$07 / 2003$

Georg-August-Universität Göttingen

Abschluss des Physikstudiums

Diplomarbeit:'Magnetische Eigenschaften von

GdN-Einfach- und GdN/Fe-Vielfachschichten'

$01 / 2001-$

Universität Uppsala, Schweden

$07 / 2001$

Auslandssemester

$07 / 2000$

Georg-August-Universität Göttingen

Vordiplom in Physik

09/1998

Georg-August-Universität Göttingen

Aufnahme des Physikstudiums 